\title{
SPECIALIZED CLATHRIN-ENRICHED SIGNALING NANODOMAINS CONTROL EPIDERMAL GROWTH FACTOR RECEPTOR SIGNALING AT THE PLASMA MEMBRANE
}

\author{
by \\ Stefanie Lucarelli \\ BSc. Biology, Ryerson University, 2014 \\ A thesis presented to Ryerson University \\ in partial fulfillment of the \\ requirements for the degree of \\ Doctor of Philosophy \\ in the program of \\ Molecular Science
}

Toronto, Ontario, Canada, 2019

(C) Stefanie Lucarelli, 2019 


\section{AUTHOR'S DECLARATION FOR ELECTRONIC SUBMISSION OF A DISSERTATION}

I hereby declare that I am the sole author of this dissertation. This is a true copy of the dissertation, including any required final revisions, as accepted by my examiners.

I authorize Ryerson University to lend this dissertation to other institutions or individuals for the purpose of scholarly research.

I further authorize Ryerson University to reproduce this dissertation by photocopying or by other means, in total or in part, at the request of other institutions or individuals for the purpose of scholarly research.

I understand that my dissertation may be made electronically available to the public. 


\title{
Specialized clathrin-enriched signaling nanodomains control epidermal growth factor receptor signaling at the plasma membrane
}

\author{
Stefanie Lucarelli \\ Doctor of Philosophy, 2019 \\ Molecular Science, Ryerson University
}

\begin{abstract}
The Epidermal Growth Factor (EGF) Receptor (EGFR) is a key receptor tyrosine kinase that controls many facets of cell physiology, including proliferation, survival and migration. EGF binding to EGFR elicits receptor phosphorylation and membrane recruitment and phosphorylation of Gab1 followed by activation of phosphatidylinositol-3-kinase (PI3K), leading to Akt phosphorylation and activation. Concomitantly upon ligand binding, EGFR is simultaneously is recruited to clathrin-coated pits (CCPs), eventually leading to receptor endocytosis. We previously uncovered that perturbation of clathrin, but not of receptor endocytosis, impairs EGF-stimulated activation of Akt signaling. Moreover, we found that some key EGFR signaling intermediates such as phosphorylated Gab1 are enriched with a subset of CCPs. We thus proposed that in addition to their well- known function as endocytic portals, some CCPs have a direct role in controlling EGFR signaling at the plasma membrane prior to receptor internalization. How CCPs and clathrin may directly control EGFR signaling at the plasma membrane prior to receptor endocytosis had remained poorly understood, which I have now examined here.
\end{abstract}

I uncovered that perturbations of TOM1L1 (a clathrin-binding regulator of Src-family kinases) and the TOM1L1-binding Src-family kinase Fyn impaired EGF-stimulated Akt phosphorylation, similarly to the effects of clathrin perturbations. A similar requirement for an 
additional cytosolic kinase Ack1 was also found. Using total internal reflection fluorescence microscopy and automated image analysis, we found that TOM1L1, Fyn, and Ack1 are enriched within a subset of CCPs, and that those that house EGFR are also those that preferentially contain Fyn or Ack1. Perturbation of TOM1L1 prevented Fyn recruitment to CCPs, and mutants of TOM1L1 defective in Fyn binding or clathrin binding abrogated Akt phosphorylation downstream of EGF stimulation. Importantly, TOM1L1- or Fyn-positive CCPs exhibit unique properties, such as distinct lifetimes, size, and recruitment of endocytic accessory proteins. Thus, these results indicate that a subset of CCPs become specialized signaling-capable structures by enrichment with key signaling regulators to control EGFR signaling, a phenomenon required for subsequent Akt activation. Given the importance of EGFR in driving growth and survival of certain types of cancer, obtaining a better understanding of how CCPs organize key EGFR signals in space and time within the PM may lead to the development of novel anti-cancer treatments. 


\section{Acknowledgments}

It's unbelievable how quickly the past five years of my life have flown by. I've imagined this day vividly for the past few years, when I get to sit down to thank all the people who have helped me get to this point, and I just can't believe it's finally here.

It seems like just yesterday I was psyching myself up to confidently walk into Costin's office (Dr. Antonescu to me back then) and ask for a volunteer position in his lab. He initially said no, and I didn't find out until years later this was a test, a way to find the students who were persistent and could prove to him that they wanted the position more than the dozens of others trying to be supervised by this wonderful professor. Costin, thank you for being the most supportive mentor and supervisor I could have ever asked for. My place in your lab has shaped me into the person I am today, as I was able to pursue what I am truly passionate about and excelled at - research. Your guidance and support over these past six years in your lab has made this whole experience worthwhile. Your positive attitude, passion for science and learning, dad jokes, and inclusiveness have all made the ANT lab such a supportive environment, and we are all so lucky to have you as our supervisor.

To Mama and Papa, I couldn't possibly begin to put into words how grateful I am for both of you. Your unconditional love and support through all of this has given me the confidence to pursue my dreams, and the reassurance that everything will turn out okay. Thank you for pushing me to be resilient in the face of adversity, and for reminding me that there is light at the end of the tunnel. How proud you both are of me makes all of this hard work worthwhile. To David and Matthew, thank you both for always making me laugh, even when I'm stressed. I don't think anyone will ever make me laugh as hard as you two can, and for that I am truly grateful. The love and support all of you give me continues to motivate me every day.

To Kaylee, can you believe this?? I guess this means we're both adults now. You have supported me through my highest highs and my lowest lows, from the tippity top to the tippity bottom, and I am so grateful every day for you. Thank you for knowing when I need to talk, for knowing when I need a drink, and for being a constant reminder that nothing is as bad as it seems. I can't imagine ever having done it without you. To Candice and Holly, your 
unconditional love and support means the world to me, not only in these past few difficult months, but throughout the course of this crazy thing I decided to do with my life. Thank you for your constant reminders of how proud you are of me and for pushing me to persevere through the tough times, as sometimes it was hard for me to see that what I was doing was all going to be worth it in the end. To the slope sisters, I can't wait to go skiing with you again soon.

To Ferris, thank you for being my motivation, my number one fan, my distraction, my shoulder to cry on, and everything else in between that you have become for me over the past 3 and a half years. Thank you for always listening to my rants about failed experiments and for celebrating the small victories with me. I love you so much, your unwavering support and confidence in me has played a monumental role in my success. To Kitty, no matter how insanely stressed I was walking into the Bellwoods mansion, and though you ignore me most of the time, you always manage to make me smile. Thank you for being so cute and fluffy.

To Taylor, Andy, Kim, Peter, Mackenzie, Sela, my life is so much better for knowing all of you. Though you may not realize, this little family that we've created has truly kept me sane in the face of intense stress. No matter the outcome of my days in lab, I always knew I had seeing all of you to look forward to, so thank you all for the constant reminder that life outside of the lab exists. Now time to make up for all the park days I've missed sitting inside studying and writing all summer.

To Stephen, Farnaz, and Victoria, we did it! Thank you all for keeping me sane throughout this last year of our PhD, I'm so thankful that I had you three to go through this with. Being able to text each other freaking out about presentations, deadlines, thesis writing, and studying has been everything. I can't wait to see what life has in store for all of us, I know I can achieve anything thanks to all of your encouragement and optimism. To John and Louis, thank you for being the most hilarious (and often most frustrating) part of my day. To Dafne, thank you for just being the absolute best, I'm so lucky to get to call you my little. You have helped me through the past few years in more ways than you can even know. To Chris, thanks for never going to trivia with me. You guys have all made grad school such an enjoyable experience, and I am going to miss our lab family so much once I graduate. It's crazy even now to think that I would look 
forward to 12 hour-long days with a million timepoints just so that we all got to hang out in lab, play music and laugh together in between.

To everyone else that has in some way shaped my experience over the past five years, I am truly grateful for each and every one of you.

To future me, you did it!! I hope this experience always reminds you that you are capable of anything. 


\section{Table of Contents}

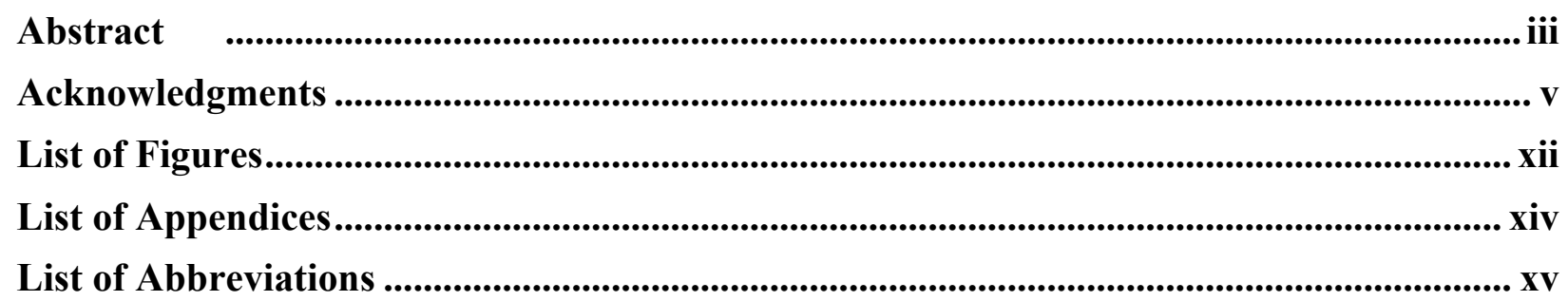

Chapter 1. Introduction ........................................................................................................................ 1

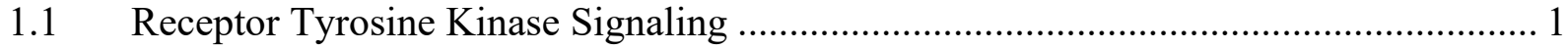

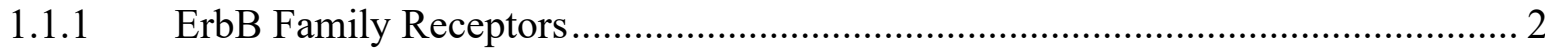

1.1.2 EGFR Activation by Ligand Binding ………................................................... 5

1.1.3 EGF Stimulated Activation of the PI3K/Akt Pathway .......................................... 6

1.1.3.1 Isoform Specific Akt Activation and Control.................................................... 9

1.1.4 Regulation EGFR by Positive and Negative Feedback Loops .............................. 11

1.1.4.1 Functional Changes Downstream of Akt Activation ....................................... 14

$1.2 \quad$ Clathrin Mediated Endocytosis............................................................................. 17

1.2.1 Clathrin Structure and Function..................................................................... 20

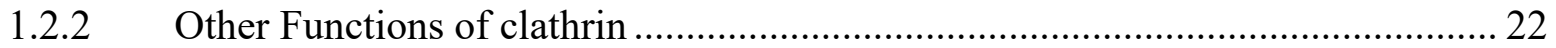

1.2.3 Adaptor proteins and cargo selection in CME ................................................. 23

1.2.4 Endocytic Accessory Proteins........................................................................ 26

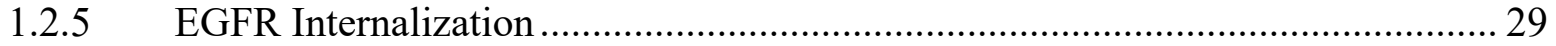

1.2.6 Clathrin Regulates Signaling and Endocytosis ................................................... 30

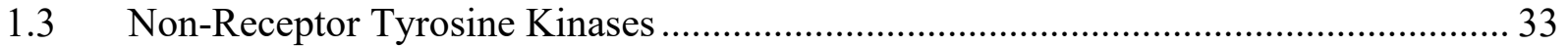

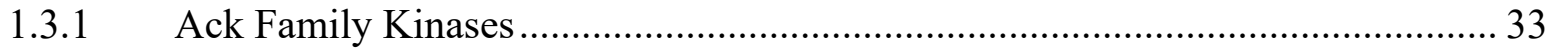

1.3.1.1 Ack1 Structure and Regulation ................................................................. 34

1.3.1.2 Ack1 Effects on Cell Physiology Mediated Through Clathrin Binding ........... 35

1.3.1.3 Aberrant Ack1 Associated with Cancer............................................................ 36

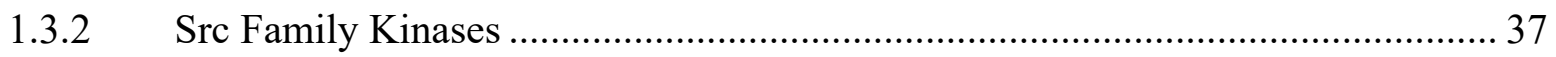

1.3.2.1 Unique cellular functions and localization of Src, Yes and Fyn....................... 39

1.3.2.2 Fyn Structure and Regulation ......................................................................... 41

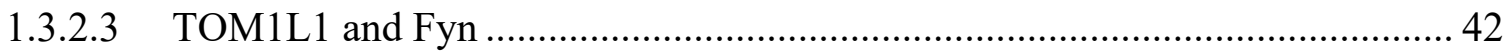

1.3.2.4 TOM1L1 is an Important Clathrin-Binding Signaling Regulator...................... 44

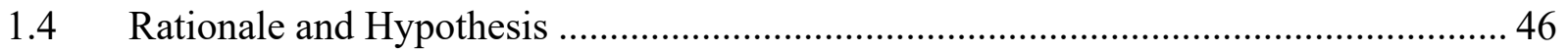


Chapter 2. Materials and Methods............................................................................... 55

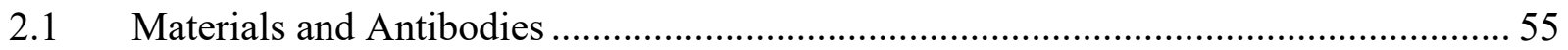

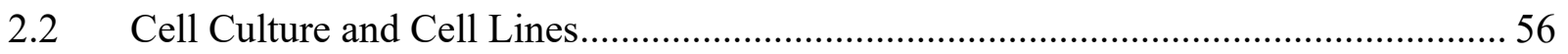

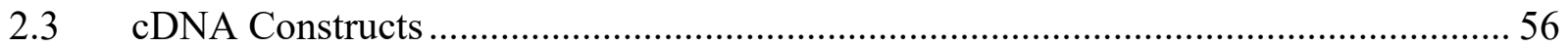

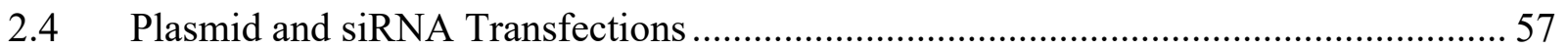

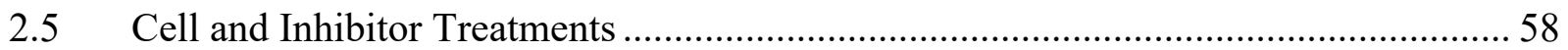

2.6 Whole-Cell Lysates and Western Blotting ........................................................... 58

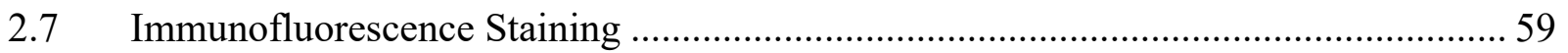

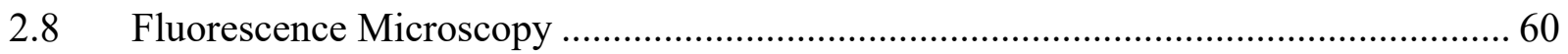

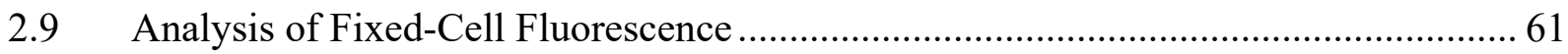

2.9.1 CLS Detection and Quantification of Protein Fluorescence Intensity ................ 62

2.9.2 Identification of EGF-positive or eGFP-Fyn positive CLSs.............................. 63

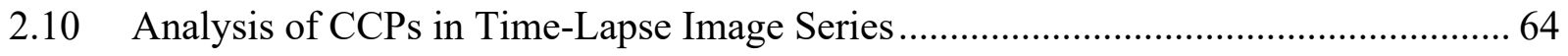

Chapter 3. Determining the capacity in which clathrin is required for receptor signaling at the plasma membrane.................................................................................................................66 6

3.1 siRNA Gene Silencing of CHC but not of Dynamin 2 Inhibits EGF-stimulated Akt

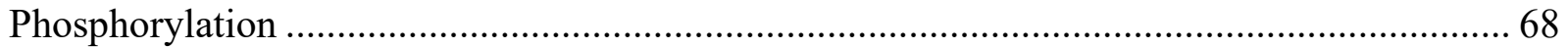

3.2 Perturbation of Clathrin by Pitstop 2 or siRNA Gene Silencing does not alter EGFR

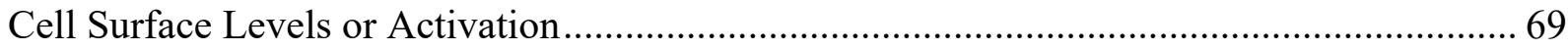

3.3 Clathrin is Required for HGF-Stimulated Akt Phosphorylation................................ 70

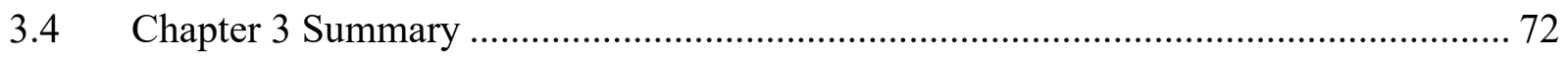

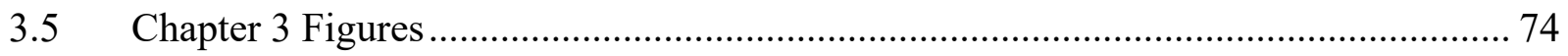

Chapter 4. The impact of non-receptor tyrosine kinase perturbation on EGFR signaling 78

4.1 Fyn is required for EGF-stimulated Gab1 and Akt phosphorylation........................ 80

4.1.1 Perturbation of SFKs by high dose Dasatinib inhibits Akt and Gab1

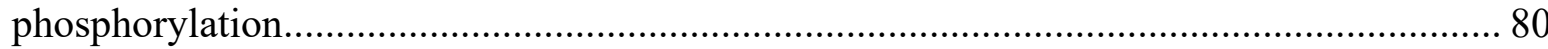

4.1.2 Perturbation of SFKs by low dose Dasatinib inhibits Src phosphorylation, but not

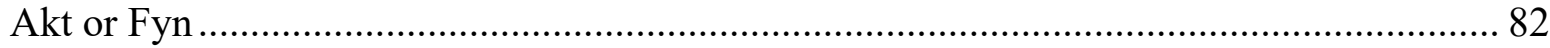

4.1.3 Fyn siRNA inhibits EGF-stimulated Gab1 and Akt phosphorylation ................ 85

4.2 TOM1L1 is required for EGF-stimulated Akt phosphorylation ................................ 86

4.2.1 TOM1L1 siRNA inhibits EGF-stimulated Gab1 and Akt phosphorylation ......... 86 
4.2.2 Fyn and clathrin binding by TOM1L1 are required for EGF-stimulated Akt

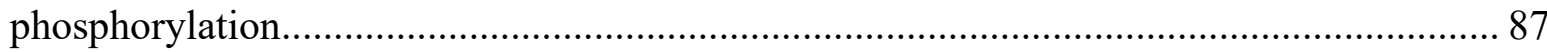

4.3 TOM1L1 or Fyn siRNA does not affect phospho-EGFR or total cell surface EGFR.. 88

4.4 Ack1 is required for EGF-stimulated Gab1 and Akt phosphorylation ........................ 90

4.5 Fyn and TOM1L1 are required for EGF-stimulated Akt phosphorylation in MCF10A cells 91

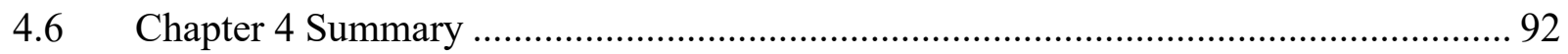

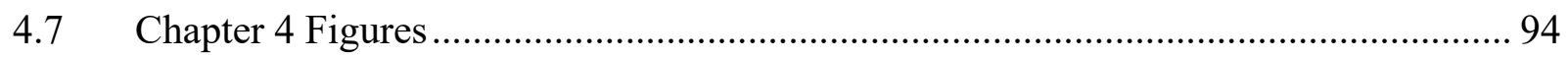

Chapter 5. A subset of clathrin-coated pits are unique signaling nanodomains required for EGFR signaling .................................................................................................................... 103

5.1 EGF and Transferrin are recruited to largely distinct CLSs ..................................... 105

5.2 Endocytic accessory proteins and kinases are enriched within a subset of plasma

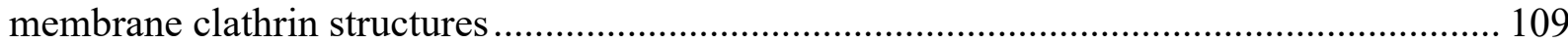

5.2.1 Fyn is enriched within a subset of clathrin structures at the plasma membrane. 110

5.2.2 TOM1L1 is enriched within a subset of clathrin structures at the plasma membrane.

5.2.3 Gab1 is enriched within a subset of clathrin structures at the plasma membrane 113

5.2.4 Ack1 is enriched within a subset of clathrin structures at the plasma membrane 115

5.3 TOM1L1 is required for recruitment of Fyn to clathrin structures............................ 116

5.4 Fyn and Ack1 are recruited to a subset of EGF-positive clathrin structures .............. 117

5.5 Fyn- or TOM1L1-positive CCPs have unique lifetimes and size.............................. 119

5.6 Fyn-positive clathrin structures contain a unique profile of endocytic accessory

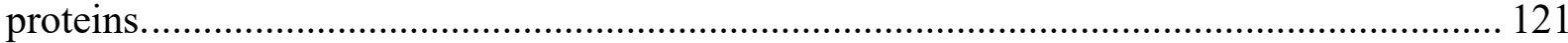

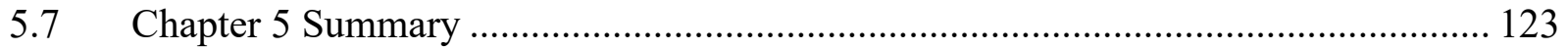

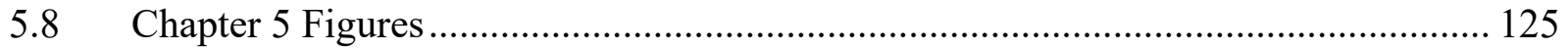

5.9 Additional Contributions ................................................................................. 142

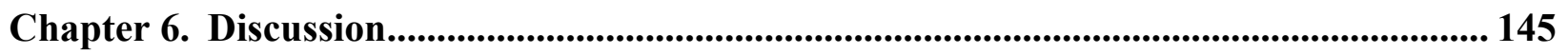

6.1 Regulation of PI3K/Akt signaling by clathrin ...................................................... 146

6.2 Regulation of PI3K/Akt signaling by Fyn and TOM1L1 ........................................ 151

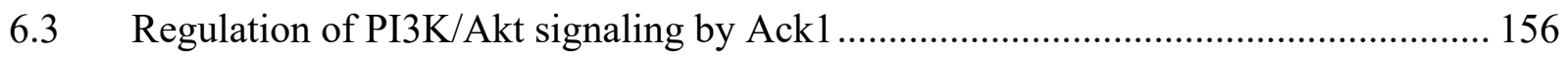

6.4 Heterogeneity of TOM1L1/Fyn function in EGFR signaling for different cell types 160

6.5 A subset of clathrin structures contain EGFR, phospho-Gab1, Ack1, Fyn, and

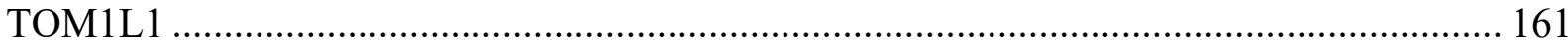


6.6 Clathrin structures that recruit Fyn or TOM1L1 have distinct properties ................ 169

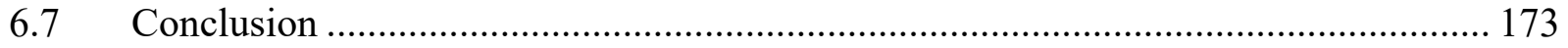

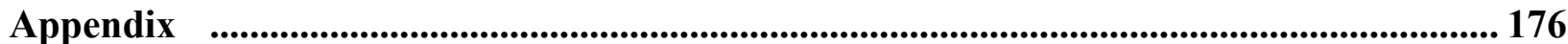

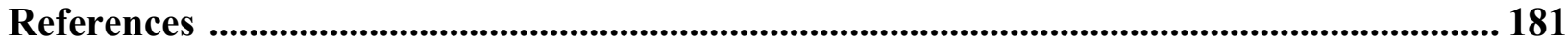




\section{List of Figures}

\section{Chapter 1}

Figure 1.1 EGF stimulation regulates EGFR membrane traffic and signaling........................ 48

Figure 1.2 Signaling pathways downstream of EGFR activation....................................... 50

Figure 1.3 EGFR activation of the PI3K/Akt pathway....................................................... 51

Figure 1.4 Mechanism of activation of Src Family Kinases.............................................. 52

\section{Chapter 3}

Figure 3.1 Silencing of clathrin but not dynamin 2 inhibits EGF-stimulated Akt phosphorylation

Figure 3.2 Perturbation of clathrin by pitstop2 treatment or siRNA gene silencing does not alter

EGF-stimulated EGFR phosphorylation............................................................................. 75

Figure 3.3 Silencing of clathrin or dynamin 2 does not affect cell surface EGFR ................... 76

Figure 3.4 Clathrin is required for HGF-stimulated Gab1 and Akt phosphorylation.................. 77

\section{Chapter 4}

Figure 4.1 High-dose Dasatinib inhibits Src and activation of the PI3K/Akt pathway.............. 94

Figure 4.2 Akt phosphorylation is less sensitive to low-dose Dasatinib than c-Src.................. 95

Figure 4.3 Fyn is required for EGF-stimulated Gab1 and Akt phosphorylation. ....................... 96

Figure 4.4 TOM1L1 is required for EGF-stimulated Akt phosphorylation.............................. 97

Figure 4.5 Fyn and clathrin binding by TOM1L1 is required for EGF-stimulated Akt

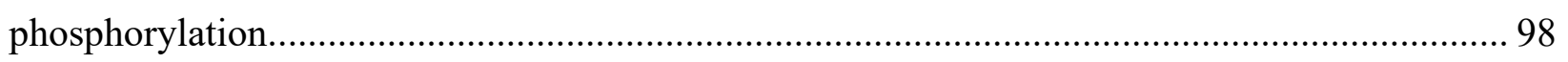

Figure 4.6 EGFR is unaffected by Fyn or TOM1L1 siRNA. ............................................. 99

Figure 4.7 Ack1 is required for EGF-stimulated Akt phosphorylation. ................................. 101

Figure 4.8 Fyn and TOM1L1 are required for MCF10A, but not SUM149PT or T47D cell EGFstimulated Akt phosphorylation. 102

\section{Chapter 5}

Figure 5.1 EGFR and Tfn are recruited largely to distinct clathrin structures. 125 
Figure 5.2 Fyn is enriched within a subset of plasma membrane clathrin structures.

Figure 5.3 TOM1L1 is enriched within a subset of plasma membrane clathrin structures.

Figure 5.4 Phosphorylated Gab1 is enriched within CCPs upon EGF stimulation.

Figure 5.5 HGF stimulation leads to an enrichment of pGab1 within CCPs.

Figure 5.6 TOM1L1 is required for Fyn recruitment to clathrin structures.

Figure 5.7 Fyn is recruited to a subset of EGF-positive clathrin structures at the plasma

membrane.

Figure 5.8 Ack1 is detected within EGFR-positive but not TfR-positive PM clathrin structures.

Figure 5.9 Fyn- or TOM1L1-positive CCPs have unique lifetimes and size.

Figure 5.10 GFP-Fyn positive clathrin structures contain a unique profile of endocytic accessory proteins. 


\section{List of Appendices}

Figure A1 Attachment of filamentous Legionella pneumophila (Lp) to lung epithelial cells (LECs) requires Src and PI3K activity.

Figure A2 Full image panels of TIRFM fluorescence micrographs shown in Figure 5.2A and Figure 5.3...... 176

Figure A3 Full image panels of SIM fluorescence micrographs shown in Figure 5.2B. 177

Figure A4 Additional representative traces of individual Fyn+ and TOM1L1+ CCPs.............. 178

Figure A5 Expression of eGFP-Fyn under the conditions examined does not alter CCP 179 


\section{List of Abbreviations}

\begin{tabular}{|c|c|}
\hline AAK1 & Adaptor associated kinase 1 \\
\hline Ack1 & Activated Cdc42-associated kinase \\
\hline ANTH & AP180 N-terminal domain \\
\hline AP2 & Adaptor protein 2 \\
\hline BAR & Bin/amphiphysin/Rvs domain \\
\hline BCL2 & B-cell lymphoma 2 \\
\hline $\mathrm{Cbl}$ & Casitas B-lineage lymphoma proto-oncogene \\
\hline CCPs & Clathrin-coated pits \\
\hline $\mathrm{CCV}$ & Clathrin-coated vesicle \\
\hline $\mathrm{CHC}$ & Clathrin heavy chain \\
\hline CIN85 & Cbl-interacting protein of 85 \\
\hline CLC & Clathrin light chain \\
\hline CLS & Clathrin labeled structure \\
\hline $\mathrm{CME}$ & Clathrin-mediated endocytosis \\
\hline CRIB & Cdc42- and Rac-interactive binding domain \\
\hline CSK & C-terminal Src kinase \\
\hline DEP-1 & Density-enhanced phosphatase 1 \\
\hline EEA1 & Early andosome antigen 1 \\
\hline EGF & Epidermal growth factor \\
\hline eGFP & Enhanced green fluorescent protein \\
\hline EGFR & Epidermal growth factor receptor \\
\hline ENTH & Epsin N-Terminal homology domain \\
\hline EPS15 & Epidermal growth factor receptor substrate 15 \\
\hline FAK & Focal adhesion kinase \\
\hline FGF & Fibroblast growth factor \\
\hline FOXO & Forkhead Box O \\
\hline Gab1 & Grb2-associated binding protein 1 \\
\hline GAT & GGA and TOM1 domain \\
\hline
\end{tabular}


GLUT4 Glucose transporter type 4

GPCR G-protein coupled receptor

Grb2 Growth factor receptor bound protein 2

GSK3 Glycogen synthase 3

HECT Homologous to the E6-AP carboxyl terminus

HGF Hepatocyte growth factor

HIP1 Huntingtin-interacting Protein 1

HSC70 Heat shock cognate $71 \mathrm{kDa}$ protein

IRS-1 Insulin receptor substrate 1

LDL Low density lipoprotein

LDLR Low density lipoprotein receptor

MAPK Mitogen-activated protein kinase

MTORC2 Mammalian target of rapamycin complex 2

NADPH Nicotinamide adenine dinucleotide phosphate

NEDD4 Neuronal precursor cell expressed and developmentally downregulated

NGF Nerve growth Factor

NMT N-myristoyl transferase

NRTK Non-receptor tyrosine kinase

NSCLC Non-small cell lung cancer

OCRL Oculo-Cerebro-Renal syndrome of Lowe

PDGF Platelet-derived growth factor

PDGFR Platelet-derived growth factor receptor

PDK1 3-Phoshoinositide-dependent kinase 1

$\mathrm{PH} \quad$ Pleckstrin homology domain

PHLPP PH domain leucine-rich repeat protein phosphatase

PI(3,4,5)P3 phosphatidylinositol-(3,4,5)-trisphosphate

PI(3,4)P2 Phosphatidylinositol-(3,4)-bisphosphate

PI(3)P Phosphatidylinositol-3-phosphate

PI(4,5)P2 phosphatidylinositol-(4,5)-bisphosphate

PI(4)P Phosphatidylinositol-4-phosphate

PI3K Phosphitidylinositol-3-kinase 


\begin{tabular}{|c|c|}
\hline $\mathrm{PKB}$ & Protein kinase B \\
\hline PLC & Phospholipase C \\
\hline PP2A & Protein phosphatase $2 \mathrm{~A}$ \\
\hline PRD & Proline-rich domain \\
\hline PSF & Point spread function \\
\hline PSP & Protein serine/threonine phosphatase \\
\hline PTB & Protein tyrosine binding domain \\
\hline PTEN & Phosphatase and tensin homolog \\
\hline PTP & Protein tyrosine phosphatase \\
\hline PTP1B & Protein tyrosine phosphatase $1 \mathrm{~B}$ \\
\hline RING & Really interesting new gene domain \\
\hline ROS & Reactive oxygen species \\
\hline RPE & Retinal pigment epithelial \\
\hline RTK & Receptor tyrosine kinase \\
\hline SAM & Sterile alpha motif domain \\
\hline SFK & Src family kinase \\
\hline $\mathrm{SH} 2$ & Src homology 2 \\
\hline SH3 & Src holomogy 3 \\
\hline SH4 & Src homology 4 \\
\hline Shc & SH2-containing collagen-related protein \\
\hline SHIP2 & SH2-domain containing inositol phosphatase 2 \\
\hline Shp2 & Src-homology 2 domain-containing phsophatase 2 \\
\hline siRNA & Small interfering RNA \\
\hline Sjn 1 & Synaptojanin 1 \\
\hline $\operatorname{Sjn} 2$ & Synaptojanin 2 \\
\hline Srcasm & Src activating and signaling molecule \\
\hline Tfn & Transferrin \\
\hline TfR & Transferrin Receptor \\
\hline TGFa & Transforming growth factor alpha \\
\hline TGN & Trans-golgi network \\
\hline TIRF-M & Total internal reflection fluorescence microscopy \\
\hline
\end{tabular}




$\begin{array}{ll}\text { TKI } & \text { Tyrosine kinase inhibitor } \\ \text { TOM1L1 } & \text { Target of Myb-1 like protein 1 } \\ \text { TSC2 } & \text { Tuberous sclerosis protein 2 } \\ \text { UBA } & \text { Ubiquitin association domain } \\ \text { UIM } & \text { Ubiquitin interacting motifs } \\ \text { VHS } & \text { Vps27p, Hrs and STAM domain }\end{array}$




\section{Chapter 1. Introduction}

The cells contained within a multicellular organism require constant communication, which is achieved through chemical signals and mechanical cues that the cell uses to sense, respond, and adapt to changing environmental conditions. The decisions that the cell makes based on the signals that it receives or sends can change the way that a cell differentiates, metabolizes, proliferates, and grows, processes that are important for development and overall healthy physiology. In order to understand these chemical signals, each cell displays a diverse complement of receptors and signaling proteins within its environment-facing plasma membrane that translate extracellular cues into intracellular changes. These changes can be as specific as the alteration of the activity of a particular gene or as broad as induction of cell division, and when these cue-sensing phenomena are dysregulated we see the onset of diseases such as cancer. The ability of a cell to translate extracellular signals is therefore dependent on the availability of cell surface receptors, without which these intracellular alterations in cell behavior cannot occur. Here, I describe the activity of a particular receptor on the plasma membrane called Epidermal Growth Factor Receptor (EGFR), part of the family of receptor tyrosine kinases (RTKs) responsible for modulation of various aspects of development, as well as tissue homeostasis and cell metabolism.

\subsection{Receptor Tyrosine Kinase Signaling}

Cell surface receptors that contain intrinsic tyrosine kinase activity are members of a family of 58 RTKs, which fall into 20 different subcategories dependent on differences in their structural domain architecture on the extracellular and cytosolic sides of the plasma membrane. 
In common between many receptors of the 20 subcategories is an extracellular region with the ability to bind various specific ligands, a transmembrane portion, an intracellular kinase domain, and a C-terminal regulatory region (Lemmon \& Schlessinger, 2010). Through binding ligand on the outside of the cell, RTKs translate extracellular cues into intracellular regulation of critical processes such as cell proliferation and differentiation, migration, and metabolism. For most RTKs, this control of cellular function occurs as a result of the cytosolic tails of these receptors becoming phosphorylated following activation, creating docking sites for phosphotyrosinebinding (PTB) domain containing proteins that couple RTK activation to downstream signaling events. Since these receptors control such important cellular processes, mutations or aberrant activation leads to a wide variety of cancers and other diseases such as diabetes, chronic inflammation, and bone disorders. EGFR and other ErbB family receptors are key RTKs implicated in many forms of human cancers, as aberrant activation or disruption in down regulation promotes cell proliferation, differentiation, and migration, ultimately driving tumor formation and cancer cell survival (Nakajima et al., 2014; Roskoski, 2014; Tomas et al., 2014). The development of therapies that block or attenuate RTK activity is therefore an active and ongoing process that requires a better understanding of the molecular mechanisms by which key RTKs control signaling events that in turn control cell behavior (Vansteenkiste \& Wauters, 2018; Yarden \& Pines, 2012).

\subsubsection{ErbB Family Receptors}

EGFR is a $170 \mathrm{kDa}$ single-pass transmembrane RTK that undergoes homo- or heterodimerization and trans-autophosphorylation upon epidermal growth factor (EGF) ligand binding. First described by Stanley Cohen (Cohen, 1983), who conducted pivotal studies on EGF 
binding and protein kinase activity of EGFR (Cohen et al., 1982; Cohen \& Carpenter, 1990) and provided the ground work for the first studies demonstrating a relationship between receptor overexpression and cancer (Thompson \& Gill, 1985). Since then, EGFR has been well studied due to its important role in various aspects of development, maintenance of tissue homeostasis, and normal cell physiology (Roskoski, 2014; Wee \& Wang, 2017).

EGFR is one of four receptor homologs belonging to the ErbB family (ErbB1-4), all of which have been found to be ubiquitously expressed in epithelial, mesenchymal, and neuronal cells (Fisher et al., 1990; Roskoski, 2014). Different members of the ErbB family have distinct sets of ligands to which they are able to bind, leading to homo- or heterodimerization with other receptor monomers and different downstream signals they are able to transmit, dependent on cell type and availability. EGF, transforming growth factor alpha (TGF- $\alpha$ ), and amphiregulin all exclusively bind EGFR; neuregulins 1 and 2 bind ErbB3 or ErbB4; heparin-binding EGF, betacellulin, and epiregulin bind to either EGFR or ErbB4; and neuregulins 3 and 4 bind to ErbB4 (Roskoski, 2014; Sweeney \& Carraway, 2000). At the cellular level, the four ErbB homologs have a critical role in regulating a variety of functions such as proliferation, differentiation, migration and apoptosis, and various ErbB receptors have distinct roles in different cell types and tissues. The production of null mutations of any of the ErbB receptor genes results in embryonic or perinatal lethality in mice, and EGFR knockout mice exhibit defects in their skin, lungs and gastrointestinal tract (Miettinen et al., 1995; Rajagopalan et al., 2008; Heidelberg, 2007; Olayioye et al., 2000). While the diversity of signaling specificity from combinations of ErbB family receptors underlies complex regulation of biological processes, this thesis focuses on EGF stimulation in cells expressing EGFR but not ErbB2, ErbB3, or ErbB4. 
This is significant as this reduces the number of signaling receptors able to bind EGF to one, allowing consistent observation of a defined, largely homogenous signaling phenomenon.

EGFR amplification or mutation and has been found to be a major contributor to aberrant signal activation in cancer cells. Upregulation of EGFR plays a large role in the growth and development of tumor cells as this receptor contributes to pathways regulating proliferation and apoptosis (Vansteenkiste \& Wauters, 2018; Yarden \& Pines, 2012). EGFR copy-number amplifications have been found in up to $52 \%$ of triple negative breast cancer cases (Nakajima et al., 2014), $15 \%$ of adenocarcinomas, and $30 \%$ of all squamous cell carcinomas, while overexpression of EGFR occurs in 60\% of all non-small cell lung cancer (NSCLC) cases (Herbst et al., 2008). It was this large proportion of NSCLCs with increased EGFR levels that drove the initial development of targeted cancer therapies that inhibit EGFR activity (Cataldo et al., 2011). The first generation of tyrosine kinase inhibitors (TKIs) targeting EGFR activity included Gefitinib and Erlotinib in 2003 and 2004, respectively (Cohen, 2005; Cohen et al., 2004), which saw an initial objective response in NSCLC patients that increased survival duration compared to the placebo-treated cohort. However, we now know that nearly all NSCLC patients with EGFRactivating mutations develop resistance to these drugs within one year of treatment (Paz-Ares et al., 2010). Aside from TKIs, therapeutic monoclonal antibodies such as cetuximab and panitumumab have been developed to bind the extracellular domain of EGFR and block ligand binding and activation in EGFR-expressing KRAS wild type metastatic colorectal cancers ( $\mathrm{Li}$ et al., 2005; Yang et al., 2001). Similarly to TKIs however, cancers treated with these targeted antibodies also eventually become resistant to them.

The efficacy of targeted drug combinations, or targeted drugs administered in combination with targeted antibody therapies is currently being explored to attempt to prevent or 
delay drug resistance in the treatment of these cancers (Roskoski, 2014; Sigismund et al., 2018).

Their limited success individually however tells us that there is still much to learn about the regulation of EGFR signaling in these cancers. Understanding how the intermediates of signaling pathways downstream of EGFR are regulated is therefore an important and ongoing part of cancer research.

\subsubsection{EGFR Activation by Ligand Binding}

Activation of EGFR requires ligand binding, which leads to receptor dimerization and autophosphorylation of the C-terminal tail. EGF contacts EGFR at two separate sites on the same receptor monomer, rather than on two separate monomers to link them together, as is the case for ligands of other RTKs like nerve growth factor (NGF) or fibroblast growth factor (FGF) (Lemmon \& Schlessinger, 2010). EGF binding to a single EGFR monomer leads to a substantial conformational change in the receptor which unmasks a dimerization motif, allowing the ligandbound monomer to form an asymmetric dimer with another monomer on the plasma membrane (Alvaradoet al., 2010; Freed et al., 2017). Dimerization activates one of the receptor's intrinsic kinase domains while the other is allosterically activated (Jura et al., 2009; Zhang et al., 2006), and multiple tyrosine residues on the C-terminal receptor tails become phosphorylated in this process. Notably, this includes Y992, Y1068, Y1086, Y1148 and Y1173 as well as others including the A loop of EGFR's kinase domain (Bromann et al., 2004) .

Phosphorylated tyrosine residues on the receptor serve as binding sites for cytosolic signaling intermediates containing domains that interact with phospho-tyrosine motifs, including Src Homology 2 (SH2) or PTB domains. In addition to autophosphorylation, Src Family Kinases 
(SFKs; in particular c-Src) participate in direct phosphorylation of EGFR at residues Y845, Y891, Y920, and Y1101, as well as enhance the phosphorylation of residues than can also be autophosphorylated, such as Y1086, Y1045 and Y1148 (Biscardi et al., 1999). C-Src mediated phosphorylation of EGFR is a key step in signal potentiation, for example EGFR Y845F mutants are impaired in their ability to synthesize DNA in response to treatment with EGF (Biscardi et al., 1999). In addition, cells expressing kinase deficient c-Src are unable to undergo phosphorylation at Y845 (along with other residues) on the receptor, resulting in decreased overall cell proliferation and tumorigenesis (Tice et al., 2002). Hence, both the direct autophosphorylation as well as c-Src mediated EGFR phosphorylation are required to trigger specific EGF-stimulated signaling events.

\subsubsection{EGF Stimulated Activation of the PI3K/Akt Pathway}

EGFR is associated with a variety of signaling pathways in the cell such as the Ras/Raf/Mitogen Activated Protein Kinase (MAPK) pathway and activation of phospholipase C (PLC), among others (reviewed in Kassenbrock et al., 2002) (Figure 1.2). One of the key pathways downstream of EGFR activation is the phosphatidylinositol-3 kinase (PI3K)/Akt pathway. Akt is a serine/threonine kinase responsible for regulation of pro-survival signals in the cell involved in proliferation, metabolism, and inhibition of apoptosis. Following ligand binding and C-terminal autophosphorylation of EGFR, the adaptor proteins Growth Factor Receptor bound Protein 2 (Grb2) and SH2-containing collagen-related protein (Shc) are recruited to the receptor. Grb2 binds EGFR directly at a motif on EGFR containing pY1068 via its SH2 domain. In addition, Grb2 provides a proline-rich domain (PRD) for the Src Holomogy 3 (SH3) domain 
of Grb2 Associated Binding Protein 1 (Gab1) to bind, which promotes the formation of a EGFRGrb2-Gab1 complex (Lock et al., 2000) (Figure 1.3).

Following binding to Grb2, Gab1 is phosphorylated at multiple tyrosine residues in a process that remains poorly understood. Phosphorylation of Y447, Y472, and Y589 on Gab1 mediates interaction with class IA PI3K at the plasma membrane (Holgado-Madruga et al., 1996; Mattoon et al., 2004) (Figure 1.3). Class IA PI3K consists of two constitutively interacting subunits: the p 85 regulatory subunit and the p110 catalytic subunit, responsible for phosphorylating membrane bound phosphatidylinositol-(4,5)-bisphosphate $\left(\mathrm{PI}(4,5) \mathrm{P}_{2}\right.$, or $\mathrm{PIP}_{2}$ from here on) at the 3 position of the inositol head group to create the lipid messenger phosphatidylinositol-(3,4,5)-triphosphate $\left(\mathrm{PI}(3,4,5) \mathrm{P}_{3}\right.$, or $\mathrm{PIP}_{3}$ from here on). The $\mathrm{p} 85$ regulatory subunit is a suppressor of PI3K catalytic activity until its $\mathrm{SH} 2$ domain binds a phospho-tyrosine motif on a target protein, relieving catalytic inhibition of the kinase (Miled et al., 2007).

The local increase in $\mathrm{PIP}_{3}$ concentration following $\mathrm{PI} 3 \mathrm{~K}$ activation acts as a signal for plasma membrane recruitment and activation of many other proteins and kinases in the cell with complementary Pleckstrin Homology $(\mathrm{PH})$ domains able to bind $\mathrm{PIP}_{3}$. Akt itself as well as 3phosphoinositide dependent kinase 1 (PDK1) are recruited to the membrane in this way via their PH domains (Figure 1.3). Akt may also be recruited to the plasma membrane and activated by its $\mathrm{PH}$ domain binding phosphatidylinositol-(3,4)-bisphosphate $\left(\mathrm{PI}(3,4) \mathrm{P}_{2}\right)$, which has been shown to occur with similar affinity to $\mathrm{PIP}_{3}$ for $\mathrm{Akt} 1$ and $\mathrm{Akt} 3$, and with less affinity than $\mathrm{PIP}_{3}$ for Akt2 (Liu et al., 2018; Sugiyama et al., 2019).

Akt is activated by two independent phosphorylation events regulated by two separate kinases; PDK1 phosphorylates Akt at T308 within the T loop of the catalytic domain (Stephens et al., 1998) and Mammalian Target of Rapamycin Complex 2 (mTORC2) phosphorylates Akt at 
S473 in a C-terminal hydrophobic motif (Alessi \& Cohen, 1998; Sarbassov et al., 2005) (Figure

1.3). Aside from phosphorylation, overall activation of Akt is dependent on the balance of negative regulatory signaling as well. Phosphorylation of Akt is subject to negative regulation by phosphatases specific for each activating site: Protein phosphatase 2A (PP2A) dephosphorylates T308 (Rodgers et al., 2011), and PH domain leucine-rich repeat protein phosphatase (PHLPP) dephosphorylates S473 (Gao et al., 2005).

The PI3K/Akt pathway as a whole is subject to negative regulation through lipid modification, notably by the lipid phosphatase and tumor suppressor Phosphatase and Tensin Homolog (PTEN) which dephosphorylates the 3 position of the inositol headgroup of $\mathrm{PIP}_{3}$ to produce $\mathrm{PIP}_{2}$ (Lee et al., 2018). Knockout mouse models of PTEN show that deletion of a single allele results in lethal autoimmune disorder phenotypes, as well as the development of different forms of epithelial cancers (deGraffenried et al., 2004). PTEN is well-known as a tumor suppressor since it is frequently found to be inactivated or mutated in a variety of cancers, including $30-40 \%$ of all breast cancer cases, resulting in hyperactivation of growth pathways including the PI3K/Akt pathway via an accumulation of $\mathrm{PIP}_{3}$ at the plasma membrane (deGraffenried et al., 2004). PTEN has also been shown to co-localize with EGFR in short-lived clathrin-coated pits (CCPs) following ligand stimulation, and to control CCP dynamics at the plasma membrane prior to internalization (Rosselli-Murai et al., 2018). Lipid modification in the $\mathrm{PI} 3 \mathrm{~K} / \mathrm{Akt}$ pathway is also mediated by the SH2-domain containing inositol phosphatase 2 (SHIP2) which dephosphorylates the 5 ' position on the inositol headgroup of $\mathrm{PIP}_{3}$ to produce $\mathrm{PI}(3,4) \mathrm{P}_{2}$ (Goulden et al., 2019). Importantly, SHIP2 is the major mechanism by which $\mathrm{PI}(3,4) \mathrm{P}_{2}$ is produced at the plasma membrane, and this occurs downstream of $\mathrm{PIP}_{3}$ production by $\mathrm{PI} 3 \mathrm{~K}$ activation (Goulden et al., 2019). Since Akt can also bind $\mathrm{PI}(3,4) \mathrm{P}_{2}$, this makes PI3K the major 
driver of all possible mechanisms of activation of Akt, and PTEN the major phosphatase that acts to negatively regulate production of $\mathrm{PIP}_{3}$.

\subsubsection{Isoform Specific Akt Activation and Control}

Akt controls an array of diverse functions in the cell, including but not limited to cell growth, metabolism, survival, protein synthesis, migration, and proliferation (Figure 1.3).

Because of its important cellular functions, it is also one of the most frequently hyperactivated kinases found in cancer, with up to $50 \%$ of human cases exhibiting promiscuous Akt activity, largely as a result of mutations to a variety of upstream regulatory signaling proteins, although mutations of Akt also contribute to aberrant Akt activation in some cancers (Gonzalez \& McGraw, 2009).

There are three isoforms of Akt, each made up of an N-terminal PH domain, a kinase domain, and a C-terminal regulatory domain (Sugiyama et al., 2019), but despite their homology, each isoform is able to control specific physiological functions from different subcellular compartments (Gonzalez \& McGraw, 2009). Notably and as mentioned earlier, the Akt isoforms exhibit differences in lipid binding affinity at the plasma membrane, and also exhibit preference for lipid binding on other endomembrane compartments in the cell: Akt1 and Akt3 are able to bind PIP3 only at the plasma membrane, and Akt2 binds $\mathrm{PI}(3,4) \mathrm{P}_{2}$ on the plasma membrane as well as on endosomes (Liu et al., 2018; Sugiyama et al., 2019). Together, the activation of each isoform regardless of cellular localization is therefore dependent on the activation of PI3K. Differences in their preferential recruitment sites reflects differences in the physiological functions and downstream effectors of each of the isoforms. Akt1 null cells exhibit high rates of 
apoptosis, indicating this isoform plays a critical role in cell survival (Chen et al., 2001; Cho et al., 2001) while Akt2 is well known to maintain cellular glucose homeostasis, as Akt2 knockout mice develop a type 2 diabetes phenotype (Garofalo et al., 2003). While the exact role of Akt3 remains unclear, knockout mice for this isoform display impaired brain development, indicating that it is involved in development here (Tschopp, 2005). Overall, the specific cellular processes under the control of each isoform are non-redundant, as simultaneous deletion of Akt1 and Akt2, or Akt1 and Akt3 cause lethality in mice either embryonically or shortly after birth (Peng et al., 2003; Yang et al., 2005). Mechanisms that control activation of PI3K are therefore important, as they impact a broad range of Akt outcomes.

There is also evidence of hyperactivation of specific Akt isoforms in certain types of cancer, suggesting that there is also isoform specific control of cellular transformation (Gonzalez \& McGraw, 2009). Akt1 has been found to be overexpressed in $24 \%$ of all breast cancer cases (Stål et al., 2003), while Akt2 gene amplification has been reported in ovarian and pancreatic cancers (Cheng et al., 2002), and Akt3 mRNA is upregulated in estrogen-receptor negative breast tumors and melanoma (Stahl et al., 2004). In many of these cases, there are no changes found in isoforms other than the hyperactivated form.

Akt has substrates in a number of intracellular compartments, including Glycogen synthase 3 (GSK3) at early endosomes and Tuberous sclerosis protein 2 (TSC2) on lysosomes (Sugiyama et al., 2019). Many of the models of Akt activation suggest that following PIP $_{3}$ binding and Akt activation, the kinase is able to dissociate from the plasma membrane and $\mathrm{PIP}_{3}$ and retain full kinase activity while it is sorted or recruited to different compartments within the cell (Sugiyama et al., 2019; Manning \& Toker, 2017). Recently however, a new study that challenged what was thought to be the mechanism of activation of Akt was published, finding 
that $\mathrm{PIP}_{3}$ binding allosterically activates $\mathrm{Akt}$ and that dissociation from $\mathrm{PIP}_{3}$ renders Akt inactive concomitantly with dissociation (Ebner et al., 2017). The ability of PH domains to recognize $\mathrm{PI}(3,4) \mathrm{P}_{2}$ as well as $\mathrm{PIP}_{3}$ may compensate for the loss of activity at the plasma membrane, as $\mathrm{PI}(3,4) \mathrm{P}_{2}$ is also detected on endosomes and lysosomes where some of the substrates of Akt are localized (Wallroth \& Haucke, 2018). In contrast to the model in which Akt is activated at the plasma membrane and then redistributed following membrane dissociation, this more recent model suggests that Akt activity is restricted to lipid membranes and is not able to dissociate into the cytosol to phosphorylate its vast array of target proteins in various subcellular localizations as was previously thought. This method of signal restriction could serve to spatially segregate signaling events, and control substrate selection.

While there still remains much to be determined about the structural mechanisms and cellular localizations that gate Akt activation and sustained signaling, this is beyond the scope of my research. One of the central themes emerging from recent studies that examined Akt signaling however is the spatial and temporal organization of Akt and the signals that lead to its activation. My work will examine the spatial and temporal organization of these signals leading to Akt activation at the plasma membrane within specific structures containing clathrin.

\subsubsection{Regulation of EGFR by Positive and Negative Feedback Loops}

Given the potency of EGFR in control of a number of physiological properties of cells and tissues, it is essential that EGFR signaling be gated and exhibit a limited activation profile upon ligand-binding in order to prevent excessive EGFR signaling outcomes. Several mechanisms of negative regulation of EGFR exist to ensure appropriate signal termination, 
including dephosphorylation of the C-terminal tyrosine residues, as well as by ubiquitination of specific lysine residues to target the receptor for proteasomal degradation. This negative feedback serves the purpose of dampening noise, increasing robustness of signaling, and preventing fluctuations in RTK activation and signaling in situations where no ligand is present. There are approximately 140 phosphatases that have been identified in the human genome that belong to one of two classes: protein Ser/Thr phosphatases (PSPs) and protein tyrosine phosphatases (PTPs) (Yao et al., 2017). SH2 domain-containing phosphatase 2 (SHP2) is one of the main PTPs involved in intracellular signaling downstream of growth factor, cytokine, and hormone receptors. Notably, Shp2 is recruited to the adaptor proteins Gab1 and insulin receptor substrate 1 (IRS-1) downstream of EGF and insulin receptors, respectively (Yao et al., 2017). The N-terminal SH2 domain of the phosphatase binds phospho-tyrosine motifs on EGFR, releasing intramolecular autoinhibition and promoting catalytic activity (Wu et al., 2001). It is also recruited to EGFR via a binding site on Grb2 following activation and promotes its dephosphorylation and negatively regulates PI3K activity downstream of EGF-stimulation by dephosphorylating Gab1 binding sites required for PI3K activation, thereby terminating the Gab1-PI3K positive feedback loop (Zhang et al., 2002). In addition to SHP2, membrane contact sites between endosomes and the endoplasmic reticulum support interaction of protein tyrosine phosphatase 1B (PTP1B) with EGFR and promotes the sequestration of EGFR on multivesicular bodies (Eden et al., 2010). Also, the tumor suppressor Density-enhanced phosphatase 1 (DEP-1) dephosphorylates EGFR at the plasma membrane, preventing its interaction with the E3 ubiquitin ligase Casitas B-lineage Lymphoma proto-oncogene (Cbl) and subsequent ubiquitination leading to lysosomal degradation (Tarcic et al., 2009). Together, the spatial 
distribution of negative feedback (EGFR dephosphorylation) regulated by these phosphatases on different endomembrane compartments control EGFR signal duration and attenuation.

Protein ubiquitination is another main way that the cell regulates protein degradation and attenuation of RTK signaling. The small $(8.6 \mathrm{kDa})$ regulatory protein ubiquitin is added to substrate proteins by the combined effort of ubiquitin-activating enzymes (E1s), ubiquitinconjugating enzymes (E2s) and ubiquitin ligases (E3s) to alter their cellular location and target them for degradation in the proteasome (Kerscher et al., 2006; Varshavsky, 1997). The two main E3 ubiquitin ligases that regulate EGFR signaling (and more generally, RTK signaling) are the Homologous to the E6-AP Carboxyl Terminus (HECT)-type ligase Neuronal precursor cell Expressed and Developmentally Downregulated (NEDD4) and the Really Interesting New Gene (RING)-type ligase Cbl. NEDD4 is a ubiquitously expressed ubiquitin ligase with 9 family members, and deletion or loss of any of which results in embryonic lethality in mice (Fouladkou et al., 2010). It was recently found that NEDD4 may act to attenuate EGFR signaling not only by ubiquitinating the receptor itself, but additionally by ubiquitinating PTEN (Trotman et al., 2007) and the kinase Activated Cdc42-associated kinase (Ack1) (Chan et al., 2009), two key accessory proteins in EGFR signaling and clathrin-mediated endocytosis (CME). Cbl is able to bind EGFR directly at pY1045 following EGF stimulation and activation, and regulates receptor internalization, intracellular trafficking, sorting and degradation. The adaptor protein Cblinteracting protein of $85 \mathrm{~K}(\mathrm{CIN} 85)$ associates with the c-terminus of $\mathrm{Cbl}$ via its $\mathrm{SH} 3$ domain, and acts as a link between the ubiquitin ligase and the endocytic accessory protein endophilin, to which CIN85 is constitutively associated, thus bringing the EGFR-Cbl complex together with the endocytic machinery of clathrin-coated pits (Soubeyran et al., 2002). This defines a regulatory role for $\mathrm{Cbl}$ in endocytosis, as translocation of endophilin to the vicinity of active EGFR 
promotes membrane invagination and receptor internalization by acting together with other regulatory proteins involved in clathrin-coated vesicle (CCV) formation (Petrelli et al., 2002; Soubeyran et al., 2002). These examples of negative regulation highlight the importance of ensuring appropriate control of EGFR signaling, and thus allowing signaling to occur only for limited periods of time following ligand binding. Sustained and robust EGFR signaling through positive feedback is achieved through a few mechanisms following ligand binding. Activation of the receptor leads to the production of $\mathrm{H}_{2} \mathrm{O}_{2}$ and other reactive oxygen species (ROS) in the cell mediated by the activation of Nicotinamide adenine dinucleotide phosphate (NADPH)-oxidase by PI3K and Rac (Bae et al., 1997). ROS are able to inhibit the activity of intracellular PTPs, causing widespread lateral activation of EGFR monomers through positive feedback. The extent and duration of this inhibition however is not known, as the cell has mechanisms in place for balancing the generation of ROS through scavenging systems, such as glutathione (Li et al., 2016).

\subsubsection{Functional Changes Downstream of Akt Activation}

Akt has over 100 substrates, including protein and lipid kinases, transcription factors, regulators of vesicle trafficking, ubiquitin ligases, and cell cycle regulators, over which Akt exerts important regulatory control. Akt preferentially recognizes the consensus sequence $\mathrm{RXRXXS/TØ} \mathrm{(where} \mathrm{X}$ is any amino acid, and $\varnothing$ is a large hydrophobic residue), however there are many context-dependent factors and secondary interactions that are often necessary to determine whether a target harboring a sequence consistent with this consensus is 
phosphorylated by Akt. I discuss some of the most critical substrates of Akt for the regulation of cell physiology next.

The first protein found to be modulated by Akt was GSK3 (Cross et al., 1995). Akt inhibits the activity of GSK by phosphorylating an amino-terminal serine residue (S21 on GSK3 $\alpha$, S9 on GSK3 $\beta$ ) that, when phosphorylated, acts as a pseudosubstrate for the substrate binding pocket of GSK3, preventing recruitment of substrates and thus the ability of GSK3 to access its substrates (Manning \& Toker, 2017). By blocking the activity of GSK3, Akt activation promotes the stability of proteins that are subject to proteasomal degradation upon GSK3 phosphorylation, such as the pro-survival B-cell lymphoma 2 (BCL-2) family member MCL-1 and the transcription factor c-Myc (Kaidanovich-Beilin \& Woodgett, 2011). Regulation of GSK3 by Akt also promotes the activity of the metabolic enzyme glycogen synthase, which is normally inhibited by phosphorylation by GSK3, and promotes more global regulation of cellular metabolism through promoting the activation of transcription factors HIF1 $\alpha$ and NRF2 (Kaidanovich-Beilin \& Woodgett, 2011).

Another notable substrate of Akt is the Forkhead Box O (FOXO) transcription factor family, which controls genes involved in fasting adaptation and insulin signaling (van der Vos \& Coffer, 2010). Activation of the PI3K/Akt pathway mediates translocation of Akt into the nucleus, where it is able to directly phosphorylate FOXO proteins, generating a recognition site for 14-3-3 proteins to bind and facilitate the exit of FOXO proteins from the nucleus and their sequestration in the cytosol (Brunet et al., 1999). By removing this transcription factor family from the nucleus, the suppression of target genes involved in induction of apoptosis, cell cycle arrest, catabolism, and growth inhibition is achieved through PI3K/Akt signaling (Manning \& Toker, 2017). In addition to FOXO, Akt phosphorylates TSC2 and inactivates it, which in turn 
leads to activation of mTORC1 thus stimulating the biosynthetic processes underlying cell, tissue, and organismal growth downstream of growth factor signaling (Manning \& Toker, 2017; Saxton \& Sabatini, 2017). Akt directly phosphorylates and inhibits TSC2, (Inoki et al., 2002) which normally acts as a GTPase activating protein (GAP) for Rheb, promoting its GDP bound (inactive) state. When inhibited however, Rheb exists in the GTP-bound state and is an essential activator of mTORC1 (Long et al., 2005).

One of the most important functions of Akt is the regulation of whole-body glucose homeostasis, mediated by Akt2. Following a meal and in response to the insulin released subsequent to a rise in blood glucose, insulin induces activation of Akt2, leading to the uptake of glucose into fat and skeletal muscle cells and inhibition of glucose production by hepatic cells (Whiteman et al., 2002). This action of insulin via activation of Akt2 results in the clearance of blood glucose to appropriate levels. To achieve this, Akt2 controls the redistribution of the Glucose transporter type 4 (GLUT4) from intracellular storage compartments to the plasma membrane, thereby increasing the flux of glucose into fat and muscle cells (Cong et al., 2014). Phosphorylation of the RabGAP AS160 by active Akt2 on at least 6 separate sites (Sano et al., 2003) inhibits its GAP activity towards Rab 8, Rab 10, and Rab 14 (Mîinea et al., 2005), preventing GTP from being hydrolyzed and rendering the Rab proteins active. These Rabs are then able to facilitate the translocation of GLUT4 to the plasma membrane where it transports glucose from the extracellular space to the cytosol for the cell to use.

From these studies, we now know that Akt in an essential kinase important for a multitude of cellular changes downstream of growth factor signaling. Thus, Akt must be robustly activated at the appropriate time, and its signaling properly controlled by negative regulation. 
There still remains much to be learned about factors that control Akt signaling, some of which will be explored in this thesis.

\subsection{Clathrin Mediated Endocytosis}

One of the most well-defined and extensively characterized methods by which cells internalize extracellular material, membrane proteins, signaling receptors, lipids, and solutes is CME. As mentioned above, CME serves to regulate EGFR and other signaling receptors by mediating internalization, which is part of a process that may lead to signal termination upon receptor degradation in the lysosome. Importantly, in addition to this, there is substantial evidence that internalization of signaling receptors such as EGFR by CME is part of a process involving endocytic membrane traffic to various endosomal compartments that in some cases potentiates or otherwise modulates receptor signaling (Schmid, 2017).

Cargo that the cell has destined for internalization is incorporated into clathrin coated pits (CCPs), small invaginating regions (80-200 $\mathrm{nM}$ in diameter) associated with the inner leaflet of the plasma membrane, enriched in the scaffolding protein clathrin and adaptor protein 2 (AP2) (Kirchhausen \& Harrison, 1981; McMahon \& Boucrot, 2011; Mettlen et al., 2018). Clathrin heavy chain $(\mathrm{CHC})$ forms a trimer called a triskelia, and these trimers self-assemble into a lattice-like structure that provides the organizational framework for vesicle formation with the help of AP2, which links clathrin to cargo and the plasma membrane via $\mathrm{PIP}_{2}$ binding. These clathrin assemblies function as protein scaffolds for recruitment of an additional 30-50 cytosolic proteins that regulate signaling and endocytosis. There are 4 main steps in CME: 1) the nucleation of a CCP by adaptor proteins and clathrin; 2) the recruitment of cargo as the CCP begins to invaginate and grow; 3 ) the maturation and eventual scission from the membrane to 
produce an intracellular $\mathrm{CCV}$; and 4) the co-ordinated disassembly of a $\mathrm{CCV}$ to ultimately deliver cargo to the early endosome (Figure 1.1).

Briefly, plasma membrane sites enriched in $\mathrm{PIP}_{2}$ and/or other endocytic cargo are able to accumulate and stabilize AP2, promoting the initial assembly of a CCP (Kaksonen \& Roux, 2018). Expression of mutants of AP2 that are deficient in either clathrin or cargo binding fail to form CCPs, or form CCPs that are unstable and rapidly abort prior to internalization (Aguet et al., 2013). This process is mediated in part by Bin/amphiphysin/Rvs (BAR) domain containing proteins, which influence membrane traffic by generating curvature at sites of their recruitment (Stanishneva-Konovalova et al., 2016). Following nucleation, adaptor proteins recognize and bind distinct sequences on the cytosolic domains of transmembrane CME cargo, recruiting them to the sites of CCP nucleation using various endocytic recognition sequences, discussed in more detail below (Traub \& Bonifacino, 2013). Following a poorly understood maturation process, the GTPase Dynamin 2 is recruited to the neck of some CCPs and is responsible for their scission from the plasma membrane, yielding an intracellular CCV. Using its PH domain and PRD, Dynamin 2 binds to $\mathrm{PIP}_{2}$ on the plasma membrane and SH3-domain containing endocytic accessory proteins found at the nucleating $\mathrm{CCP}$, such as amphiphysin and endophilin (Mousavi et al., 2004). Here, Dynamin self-assembles into a helicoidal structure that constricts the membrane, forming it into a tubular shape. Dynamin self-assembly activates the GTPase activity of dynamin, and GTP hydrolysis at this site induces a conformational change in Dynamin that results in local tightening of the helix surrounding the tubule, eventually causing scission of this tubule from the membrane (Antonny et al., 2016; Chappie et al., 2011; Kozlov, 1999).

The average lifetime of a $\mathrm{CCP}$ at the plasma membrane, from nucleation to scission, is approximately 20-60 seconds (Aguet et al., 2013; Ehrlich et al., 2004; Jaqaman et al., 2008; 
Loerke et al., 2009). As reflected by the heterogeneity in their lifetimes (which spans nucleation, cargo recruitment, maturation and scission, as outlined above), these structures are highly heterogeneous in accessory protein content, and we are still trying to elucidate the functional applications of this property. In addition to this heterogeneity of lifetimes and composition, there is also heterogeneity of fate of the CCPs after initiation, as some structures do not proceed to formation of CCVs. In normal mammalian cells, only about $40 \%$ of CCPs are able to successfully bud into the cell from the plasma membrane; the other $60 \%$ of clathrin structures represent abortive populations with lifetimes ranging from 5-15 seconds at the plasma membrane followed by disassembly of its recruited components (Loerke et al., 2009). It is not yet clear what exactly causes a clathrin structure to become abortive, and whether there is a size or cargorelated checkpoint that must be met in order to proceed to maturation and scission, however there are a number of factors known to contribute. Overexpression or clustering of the Transferrin Receptor (TfR) increases the proportion of productive CCPs relative to abortive events (Liu et al., 2010; D Loerke et al., 2009), however silencing using small interfering RNA (siRNA) of the endocytic accessory proteins clathrin assembly lymphoid myeloid leukemia protein (CALM) or epsin serves to increase the proportion of abortive CCPs (Mettlen et al., 2018; Mettlen et al., 2009).

Following internalization, the CCV is rapidly uncoated with the aid of the uncoating ATPase Heat shock cognate $71 \mathrm{kDa}$ protein (HSC70) and the J-domain containing proteins auxillin (nerve-specific) and its homolog GAK (ubiquitous), so that clathrin can be recycled and used in additional endocytic events in the cell (Morgan et al., 2001; Park et al., 2015). Auxillin or GAK first binds the vertex of CHC on a newly internalized CCV and then recruits HSC70 to the site (Fotin et al., 2004). HSC70 hydrolyzes ATP to ADP which leads to tightening of its 
"molecular clamp" domain that contacts the carboxy-terminal segment of a clathrin molecule, eventually leading to a local distortion in the clathrin coat that when accumulated at multiple vertices causes coat dissolution (Kirchhausen et al., 2014; Ungewickell et al., 1995).

Stoichiometric amounts of HSC70 are required to dissociate clathrin: three molecules of HSC70 per clathrin triskelia, and three molecules of ATP hydrolyzed (Barouch et al., 1994). In addition to the roles of HSC70 and auxillin, the inositol phosphatases Synaptojanin 1 and 2 (Sjn1, Sjn2) deplete CCVs of $\mathrm{PIP}_{2}$ which weakens the affinity of AP2 for the vesicle membrane, promoting dissociation and uncoating (Kirchhausen et al., 2014; Verstreken et al., 2003). Following uncoating, internalized cargo is trafficked through the endomembrane system, and it is targeted for either ubiquitin-dependent proteolytic degradation in the lysosome through trafficking to the late endosome, or it is targeted for recycling back to the plasma membrane depending on the current metabolic needs of the cell. Both of these processes occur approximately $30 \mathrm{~min}$ to hours following ligand stimulation.

\subsubsection{Clathrin Structure and Function}

A clathrin subunit is a trimeric structure, composed of three $\mathrm{CHCs}$ (required) and three clathrin light chains (CLCs) (regulatory) organized into a three-legged triskelia structure (Kirchhausen \& Harrison, 1981). Individual clathrin triskelia are able to self-assemble into polyhedral lattice-like cages on membranes, in a process regulated by a wide variety of clathrin binding and adaptor proteins. Purified clathrin is also able to self-assemble into empty cages at pH values below 6.8 (Kirchhausen \& Harrison, 1981). This cage-like structure is defined by a lattice of pentagons and hexagons formed by polymerization of individual clathrin triskelia in 
such a way that the center of the triskelia represents a vertex of the lattice. Differences in the number of pentagons and hexagons formed influences the curvature of a clathrin structure, and defines two broad, distinct populations: classic coated-pits, containing a mix of pentagonal and hexagonal shapes, and largely hexagonal flat clathrin lattices sometimes referred to as "plaques" (Brodsky, 2012). These clathrin plaques are ubiquitous, stable, heterogeneous structures that persist on the plasma membrane for hours, displaying little change in position or morphology, and exhibit sustained dynamin recruitment (Grove et al., 2014). The functional relevance of clathrin plaques remains unclear, however they may act as membrane scaffolds or microdomains for processes occurring at the plasma membrane (Leyton-Puig et al., 2017), which is a function of clathrin that I will discuss later.

There are two main isoforms of $\mathrm{CHC}$ : $\mathrm{CHC} 17$, which is ubiquitously expressed in all tissues and regulates processes including CME, lysosome biogenesis, and endosomal sorting, and CHC22, which mediates retrograde transport from endosomes to the trans-golgi network (TGN) in skeletal muscle (Royle, 2006). Both isoforms of CHC are structurally comprised of two well defined regions. First, a long repeating alpha helix region arranged in antiparallel helix-turn-helix motifs called the $\alpha$-solenoid region, makes up the ankle, distal leg, knee, and proximal leg segments of CHC, and also contains the trimerization domain (Fletterick et al., 1999). The second structural component of $\mathrm{CHC}$ is a single seven-bladed $\beta$-propeller domain, which is mainly responsible for binding adaptor and accessory proteins through four different key binding sites. One well characterized site, known as the clathrin box, has a consensus sequence of $\mathrm{L} \varnothing \mathrm{X} \varnothing[\mathrm{DE}]$ (Ø represents a hydrophobic residue, alternative residues in brackets) and alone is responsible for binding amphiphysin, $\beta$-arrestin 1 , and AP2, which are all required for CME. The well-studied small molecule inhibitors of clathrin mediated endocytosis, Pitstops 1 and 2, bind 
the clathrin box and interfere with normal adaptor and accessory protein interaction with $\mathrm{CHC}$ (Von Kleist et al., 2011). Another interaction site in the N-terminal domain binds W-box motifs (PWXXW) such as those found in amphiphysin (Miele et al., 2004). CHC22 is expressed mainly in muscle and fat where it is required for the intracellular trafficking of glucose transporters (Dannhauser et al., 2017; Vassilopoulos et al., 2009). In contrast, CHC17 triskelia mediate the canonical functions associated with clathrin, including receptor-mediated endocytosis and lysosome biogenesis (Dannhauser et al., 2017), which is why for the purpose of this thesis, I will be considering only this isoform.

There are two main isoforms of clathrin light chain as well: CLCa and CLCb, which both associate exclusively with $\mathrm{CHC} 17$ (Liu et al., 2001) with high affinity ( $\left.\mathrm{Kd}=10^{-9} \mathrm{M}\right)$. Both isoforms contain a 100\% conserved N-terminal region, an HSC70 binding site (CLCa only), a calcium binding domain, a heavy chain binding site, and a C-terminal calmodulin-binding domain (Royle, 2006). Unlike CHC, the functional relevance of CLC remains poorly understood, however it has been proposed to regulate assembly of clathrin triskelia though interaction with various factors such as calcium (Wu et al., 2016). CLC is only one of the many mechanisms by which clathrin assembly into CCPs is regulated. Additional mechanisms include many other endocytic accessory proteins that associate with clathrin, which will be examined below.

\subsubsection{Other Functions of clathrin}

Clathrin is one of three main coat proteins along with COPI and COPII used in the transport of select proteins and lipids between distinct cellular compartments of the cell. Each of these proteins self-assemble with associated adaptor proteins on a distinct subset of intracellular 
membranes and function in sequestering and sorting cargo to be transported through the formation of coated membrane patches. Clathrin functions in sorting proteins at the plasma membrane, endosomal membranes, and the trans-golgi network (TGN) which aids in vesicle budding to assist in processes such as organelle biogenesis, secretory granule formation, antigen presentation, glucose transport sequestration, and synaptic vesicle generation (Brodsky, 2012; Dell'Angelica, 2001). The clathrin coats that assemble on each of these distinct membranes share a single reservoir of soluble cytosolic clathrin, meaning that there are compartment-specific adaptors responsible for recruiting clathrin to different sites of action.

\subsubsection{Adaptor proteins and cargo selection in CME}

There are three main hubs of clathrin mediated endocytosis: CHC (as described above), $\mathrm{PIP}_{2}$ in the plasma membrane, and adaptor proteins. Importantly, adaptor proteins are critical for linking clathrin to the plasma membrane since clathrin cannot associate with membranes directly. AP1 and AP2 are members of this family, and mediate vesicle formation from the TGN and from the plasma membrane respectively. AP3 is also able to associate with clathrin, and is involved in signal-mediated protein sorting to endosomal-lysosomal organelles (Dell'Angelica et al., 1998; Kural et al., 2012). The final two family members, AP4 and AP5 do not appear to link to clathrin as AP1-3 do. Since my work will focus on the role of clathrin at the plasma membrane, I will focus on AP2 when discussing adaptor proteins and CCV formation. AP2 is a stable tetramer complex composed of four distinct subunits: $\alpha$ and $\beta 2$ adaptins, $\sigma 2$, and $\mu 2$ (Collins et al., 2002). Each of the two large subunits ( $\alpha$ and $\beta 2$ ) consist of an N-terminal trunk domain and a $\mathrm{C}$-terminal appendage domain connected by a flexible hinge. The $\alpha$ appendage 
domain allows AP2 to interact with AP180, amphiphysin, and other endocytic accessory proteins via a DPF or DPW motif (Mousavi et al., 2004). To directly associate with clathrin, there is a clathrin box motif found on the $\beta 2$ hinge region of AP2, as well as a second binding site in the $\beta 2$ appendage domain (Dell'Angelica et al., 1998). AP2 is the most abundant non-clathrin protein found in purified CCVs, and is the prototypical and most well understood adaptor protein of the family. As such, the ability of AP2 to regulate and trigger CCP formation must be tightly regulated, which is achieved by several mechanisms, as discussed next.

To be recruited into a budding CCP, a cargo protein must display a sorting signal that is preferentially bound by an adaptor protein that links the cargo to the clathrin machinery. The $\mu 2$ domain of AP2 recognizes cargo via a YXXØ motif (X is any amino acid and $\varnothing$ is a bulky hydrophobic amino acid) (Kadlecova et al., 2017; Ohno et al., 1995; Owen, 2002). The ability of $\mu 2$ to recognize cargo in this way however is dependent on its own phosphorylation at Thr156 by adaptor associated kinase 1 (AAK1) (Olusanya et al., 2001), and stabilization of its open binding conformation by simultaneous $\mathrm{PIP}_{2}$ binding on the plasma membrane. This motif recognizes such cargo as TfR, PAR1, LAMP1, Dishevelled 2, and GABA receptor $\gamma 2$ (Traub, 2009). Depletion of AP2 by siRNA leads to a complete reduction of TfR internalization (Motley et al., 2003). Another important sorting signal recognized by AP-2 is the dileucine motif [DE]XXXL[LIM], the interaction interface of which is found this time on the trunk domain of the $\beta 2$ subunit. This motif does not compete for entry with the YXXØ motif, and includes proteins such as CD4, LIMP2, and Nef (Traub, 2009).

Although for a long time AP2 was assumed to be absolutely required for clathrinmediated endocytosis of the vast majority of cargo proteins, it was surprising that depletion of the alpha subunit of AP2 does not affect the uptake of certain cargo into CCVs (Motley et al., 
2003). Importantly, clathrin itself remained essential under these conditions. This observation, coupled with the discovery that some accessory proteins that bind AP2 are also able to bind $\mathrm{PIP}_{2}$ and clathrin, led to the hypothesis that there are alternative adaptor proteins that aid in recruitment and internalization of cargo at the plasma membrane independently of, or in addition to, AP2. It remains unclear if depletion of individual AP2 subunits indeed generates CCPs that are devoid of AP2 entirely, as some have proposed that deleting subunits of AP2 permits the formation of hybrid adaptor protein complexes that resemble endogenous, complete AP2 but lack specific functions of AP2 (Gu et al., 2013). It is thus not clear if CCPs can form in the complete absence of AP2 or related adaptor proteins, or whether a small amount of partially functional AP2 is required to trigger initiation of all CCPs that may then mediate internalization of specific cargo. Indeed, some of the so-called alternative adaptor proteins (which can bind $\mathrm{PIP}_{2}$ and clathrin) also display unique cargo recognition and are often responsible for the uptake of one specific type of cargo over others, which I examine next.

Dab2, ARH, and NUMB are all members of the tyrosine-binding domain-containing adaptor proteins that recognize the $[\mathrm{FY}] \mathrm{XNPX}[\mathrm{YF}]$ motif on cargo, a motif that is not recognized by AP2 (Traub, 2009). Dab2 mediates the uptake of low density lipoprotein (LDL) and its receptor (LDLR) and the scavenger receptor Megalin in most cell types independently of ARH and AP2, however if Dab2 is depleted, LDL and LDLR are internalized via ARH in an AP2 dependent manner (Maurer \& Cooper, 2006).

Another group of alternative clathrin adaptors that may be particularly important for internalization of signaling receptors such as EGFR recognize ubiquitin as a sorting signal for CME. Many signaling receptors, ion channels and transporters that are not constitutively internalized use this signal, which requires a post-translational modification via ubiquitination to 
tag it for uptake. Epsin 1, epsin 2, and epidermal growth factor receptor substrate 15 (EPS15) are all members of this class of adaptor proteins, and recognize tandem ubiquitin interacting motifs (UIMs) on target proteins, which include EGFR and MHC class I (Hicke \& Dunn, 2003; Traub, 2009; Wendland, 2002). Additionally, the arrestin family of proteins has a well-established role in the internalization of many $\mathrm{G}$ protein-coupled receptors (GPCRs) following ligand stimulation and hyperphosphorylation of the $\mathrm{C}$-terminal domain (Marchese et al., 2007). $\beta$-arrestin is recruited to and binds phosphorylated GPCRs at the plasma membrane, destabilizing its basal inactive state and unmasking an AP2 interacting motif that links the GPCR to the CME machinery.

\subsubsection{Endocytic Accessory Proteins}

In addition to the alternate clathrin adaptors, there are well over 50 known cytosolic proteins that competitively bind clathrin or AP2, and control different aspects of endocytosis such as membrane bending, membrane scission, vesicle formation, cytoskeleton interaction, and vesicle uncoating (Dell'Angelica, 2001). A comprehensive discussion of all these so-called endocytic accessory proteins is beyond the scope of this thesis, but below (and in Table 1) I discuss some key functions mediated by specific accessory proteins relevant to this research:

(i) Regulation of membrane curvature. Some key accessory proteins that function in CME include AP180/CALM, which contains an AP180 N-terminal (ANTH) domain that mediates their binding to $\mathrm{PIP}_{2}$ containing membranes (Dell'Angelica, 2001). Additionally, epsin is an important curvature-inducing endocytic accessory protein which contains an Epsin N-Terminal 
Homology (ENTH) domain that binds PIP $_{2}$ at the plasma membrane (Ford et al., 2002). Upon $\mathrm{PIP}_{2}$ binding, the N-terminal helix of epsin's ENTH domain inserts into the plasma membrane and drives curvature by acting as a wedge that displaces lipids contained there (Horvath et al., 2007; Sochacki et al., 2017). Both AP180 and ENTH-domain containing proteins like epsin also contain disordered domains that lack three-dimensional structure, but potently induce curvature through membrane crowding producing steric pressure (Busch et al., 2015). BAR domaincontaining proteins such as amphiphysin and endophilin also induce membrane curvature during CME (Gallop et al., 2006; Mim \& Unger, 2012; Scott et al., 2018). That there are many different mechanisms of generating membrane curvature suggests there is the possibility of heterogeneity in how individual CCPs attain membrane curvature.

(ii) interaction with the cytoskeleton: Huntingtin-interacting Protein 1(HIP1) and its homolog HIP1R are able to directly bind CLC and actin (Chen \& Brodsky, 2005; Le Clainche et al., 2007; Legendre-Guillemin et al., 2005), and regulate actin dynamics during endocytosis.

Overexpression of HIP1 causes alterations in the actin cytoskeleton, generating short protrusions tipped with cortactin (Chen \& Brodsky, 2005), suggesting that disruption of HIP1 interactions with the cytoskeleton has detrimental effects on the cytoskeleton. In addition, HIP1R mediates the positioning of actin assemblies on budding vesicles (Le Clainche et al., 2007). Intersectin is thought to aid in linking the clathrin endocytic machinery to the actin cytoskeleton as well. Intersectin has been described to bind numerous components of the clathrin endocytic machinery including dynamin, AP2, FCHo1/2, and epsin (Henne et al., 2010; Sundborger \& Hinshaw, 2014; Wong et al., 2012), and also to stimulate actin filament nucleation and polymerization by activating N-WASP (Gryaznova et al., 2015). 
(iii) lipid enzymes: Sjn 1 and 2 are phosphoinositide phosphatases that are able to interact with a variety of endocytic accessory proteins including endophilin, amphiphysin, and intersectin (Cestra et al., 1999; De Heuvel et al., 1997; Slepnev \& De Camilli, 2000). Their N-terminal SacI domain primarily dephosphorylates phosphatidylinositol-3-phosphate (PI(3)P) and phosphatidylinositol-4-phosphate (PI(4)P), while the 5-phosphatase domain dephosphorylates $\mathrm{PIP}_{2}$ and $\mathrm{PIP}_{3}$ at the 5' position of the inositol ring, in turn regulating $\mathrm{CME}$ at the plasma membrane through lipid remodeling (Guo et al., 1999; McPherson et al., 1996; Wenk et al., 2001). Additionally, the 5-phosphatase Oculo-Cerebro-Renal syndrome of Lowe (OCRL) directly interacts with clathrin and regulates CME (Choudhury, 2005; A. Ungewickell et al., 2004). Cells lacking OCRL taken from patients with Lowe syndrome exhibit a 1.5 fold increase in steady state levels of $\mathrm{PIP}_{2}$, and exhibit defects in CME by an accumulation of late-stage Ushaped CCPs at the plasma membrane (Nández et al., 2014). Since the substrates of these lipid phosphatases are concentrated at the plasma membrane, and since $\mathrm{CME}$ requires $\mathrm{PIP}_{2}$, the actions of these enzymes here directly alter CME.

(iv) possible regulators of signaling: AAK1 directly binds and phosphorylates AP2 (Conner \& Schmid, 2002), which is required for TfR endocytosis (Olusanya et al., 2001). This phosphorylation event is promoted by clathrin assembly, indicating a positive feedback loop in which CCP maturation is facilitated (Conner et al., 2003). Its action at the site of CCP nucleation suggests that it may also regulate the phosphorylation of other signaling effectors found here. Ack1 is another kinase involved in CME, as it is required for degradation of multiple RTKs including EGFR (Shen et al., 2007), as well as modulation of RTK signaling processes such as 
cell migration, adhesion and spreading (Prieto-Echagüe \& Miller, 2011). Ack1 is able to directly bind clathrin and influence clathrin assembly (Taylor et al., 2011; Teo et al., 2001), and is also able to bind EGFR upon EGF stimulation (Shen et al., 2007).

Although the precise role and timing of many of these accessory proteins in CME remains poorly understood, one common feature shared by many of them is the ability to bind the core endocytic components clathrin, $\mathrm{PIP}_{2}$, or AP2. Since these proteins compete for a limited number of binding sites on clathrin and AP2, individual CCPs are expected to be compositionally distinct, each recruiting and binding different assortments of cytosolic proteins, which has indeed been observed for the composition of CCPs (Antonescu et al., 2011; Taylor et al., 2011). As discussed above, this compositional heterogeneity of CCPs may be reflected in the heterogeneity of CCP lifetimes and other parameters. One of the keys to understanding how clathrin functions as a signaling scaffold is to understand how EGFR and clathrin might coordinate the dynamic recruitment of specific cytosolic effectors (such as non-receptor tyrosine kinases (NRTKs)) to CCPs in order for signaling to occur.

\subsubsection{EGFR Internalization}

EGFR is often used as an example of a protein that can undergo ubiquitin-dependent internalization, however it also contains both a YXXØ and diLeucine sorting motifs in its cytosolic domain recognized by AP2 (Traub, 2009), and its overall method of recruitment and internalization through CME remains unclear. EGF stimulation also leads to receptor ubiquitination by the E3 ubiquitin ligase $\mathrm{Cbl}$, which has been shown to result in accelerated 
receptor down regulation through endocytosis (Levkowitz et al., 1999) and targeting of receptors for lysosomal degradation, though this may not be absolutely required for endocytosis.

Depletion of AP2 by siRNA gene silencing leads to a complete reduction of TfR internalization (Mettlen et al., 2010b), however, there are various studies that show differing results for EGFR endocytosis. Following AP2 depletion by siRNA in HeLa cells, small nonstoichiometric amounts of the $\alpha$ subunit alone were sufficient for normal internalization of EGFR in an EGF-stimulated and kinase-dependent manner (Johannessen et al., 2005). Collectively, these studies suggest that the mechanisms of recruitment of EGFR to CCPs by AP2 differs from that of other non-signaling receptors (e.g. TfR). This also suggests that given the ability of specific cargo receptors to regulate the properties of CCPs in which they are contained, that EGFR-containing CCPs are distinct from those containing other receptors, but this has not been resolved. The exact cocktail of proteins and their respective timing required for the internalization of EGFR has not yet been elucidated, and may differ depending on the cell type and needs of the cell.

\subsubsection{Clathrin Regulates Signaling and Endocytosis}

CME has most commonly been regarded as a means of signal attenuation for activated cell surface receptors, however this mechanism can also have signal-propagating functions. Receptors internalized from the plasma membrane are trafficked to the early endosome where those destined for desensitization are sorted to the late endosome and lysosome for degradation, and those for which the cell requires resensitization are shuttled back to the plasma membrane for sustained signaling (Platta \& Stenmark, 2011). Activated receptors do not stop signaling once 
they are internalized however, as signaling is not restricted to the plasma membrane and may take place actively while the receptor shuttles through the endo-lysosomal system. Unique signaling events may also occur preferentially on endosomes, as the membranes of these structures are functionally distinct from that of the plasma membrane and recruit its own complement of proteins (Lemmon, 2008). Early endosomes in particular are enriched in PI(3)P, and recruit proteins that contain PI(3)P-interacting domains, like FYVE or PX (Funderburk et al., 2010; Lemmon, 2008).

Trafficking of EGFR from the plasma membrane through the endomembrane system provides EGFR with a series of distinct platforms enriched with unique signaling effectors and lipid signatures that promote the activation of distinct signaling pathways. Indeed, we see that expression of a dominant negative form of dynamin blocks EGFR internalization from the plasma membrane, however signaling to MAPK and Akt is largely unperturbed (Sousa et al., 2012; Vieira et al., 1996). Activation of Akt and Erk at endosomes can also occur (Wang et al., 2002b), and many signaling effectors that are found at the plasma membrane with active EGFR are also co-internalized and localize to early endosomes where they sustain signaling there from (Di Guglielmo et al., 1994; Haugh et al., 1999; Wada et al., 1992). Together, these studies suggest that the view that endosomal signaling platforms are required for EGFR signaling, in particular for Akt phosphorylation, need to be revised, as these pathways are fully activated in the absence of endocytosis (Goh et al., 2010; Sousa et al., 2012). Together, this suggests that signaling to PI3K-Akt happens at the plasma membrane and any role that clathrin may have in this process could be separate from or precede the role of clathrin in endocytosis of EGFR.

Recent studies have shown that unique signaling events occur during receptor residence at the plasma membrane within CCPs, suggesting a role for clathrin in signaling and not just 
endocytosis (Eichel et al., 2016; Garay et al., 2015). Previous work from our lab has examined the effect of perturbation of clathrin with Pitstop, a small molecule drug that prevents the interaction of cytosolic proteins with the N-terminal domain of CHC. This inhibitor is well known to prevent the formation of new CCPs at the membrane and to ablate the recruitment of specific cytosolic proteins such as amphiphysin to CCPs (Von Kleist et al., 2011). Treatment of cells with Pitstop 2 results in decreased EGF-stimulated phosphorylation of Gab1 and Akt, but not phosphorylation of EGFR itself (Garay et al., 2015). In contrast, inhibition of dynamin 2, which allows for the formation of CCPs but not their internalization from the plasma membrane, increased the potentiation of EGF-stimulated Gab1 and Akt phosphorylation (Garay et al., 2015). This suggests that residence within CCPs and clathrin itself present the receptor with a unique signaling environment essential for signaling at the level of Gab1 phosphorylation downstream of EGFR. This study leaves a few important questions to be examined, including whether this role for clathrin can be confirmed by other more specific mechanisms (eg. siRNA), and what is the mechanism is by which clathrin controls Gab1-PI3K-Akt signaling.

It remains poorly understood which NRTKs are responsible for phosphorylating Gab1 leading to Akt phosphorylation, but it has been demonstrated that this phosphorylation event requires clathrin (Garay et al., 2015). Some specific NRTKs, like Ack1, are able to bind clathrin directly and therefore are of interest to study to further understand the regulation of Gab1 phosphorylation in the Akt pathway. If and how Ack1 may regulate clathrin-dependent signaling following EGF-stimulation is a topic that will be explored in this thesis. 


\section{$1.3 \quad$ Non-Receptor Tyrosine Kinases}

In humans there are more than 500 characterized protein kinases, with 90 of them specific for phosphorylation of tyrosine residues (Roskoski, 2014). The tyrosine kinases are further divided into two major categories; RTKs (such as EGFR) and NRTKs (such as Src or Ack1). Protein kinases in general play an important role in many aspects of cellular function including apoptosis, cell cycle progression, cytoskeletal rearrangement, and metabolism through amplification of signaling events and propagation of numerous signaling pathways. It is for this reason that their dysregulation can have severe consequences such as cancer and varieties of other disorders. There are 10 families of mammalian NRTKs including Src, Abl, Jak, Ack, Csk, Fak, Fes, Frk, Tec, and Syk (Prieto-Echagüe \& Miller, 2011). This thesis is focused on members of the Src and Ack family kinases, given what is known about the ability of specific members of these families to associate with EGFR and/or clathrin, and thus are important candidates for the mechanism by which clathrin may support EGFR signaling to Akt, as discussed below.

\subsubsection{Ack Family Kinases}

The Ack family of NRTKs consist Ack1/TNK2, Ack2 and Tnk1 in humans, along with the homologs DACK (drosophila), ARK-1 (c. elegans), DPR2 (drosophila), and Kos1 (murine). The Ack family of kinases are unique in the fact that they are the only family of NRTKs with SH3 domains located C-terminal to their kinase domain (Prieto-Echagüe \& Miller, 2011). They are also the only known tyrosine kinases to contain a CRIB (Cdc42/Rac Interactive Binding) domain. The seven kinases within the Ack family share similar core sequence beginning at the 
N-terminus and spanning the sterile alpha motif (SAM), kinase, and SH3 domains, but differ in sequence length and domain architecture past this point.

Ack1 was first isolated from a human hippocampal library by its ability to bind GTPbound Cdc42 specifically, but not Rho or Rac (Manser et al., 1993). It is ubiquitously expressed throughout the body, with highest expression levels detectable in the brain, spleen and thymus, and is constitutively tyrosine phosphorylated even in the absence of serum (Galisteo \& Yang, 2006). Stimulation of Ack1 with EGF has been demonstrated to result in a 2.5-3 fold overall increase in the phosphorylation of the kinase at Y284 (Chan et al., 2011; Galisteo \& Yang, 2006). Aside from this, Ack1 has been found to be detectably phosphorylated at Y284 in response to stimulation with bradykinin (Yang \& Cerione, 1997), platelet-derived growth factor (PDGF), and insulin (Galisteo \& Yang, 2006), as well as in response to integrin mediated cell adhesion (Galisteo \& Yang, 2006). Ack1 is also required for degradation of multiple RTKs, as well as modulation of processes such as cell migration, adhesion and spreading (Prieto-Echagüe \& Miller, 2011). Despite these studies, much about Ack1 remains to be determined including its contribution to RTK signaling from the plasma membrane, which will be part of the focus of this thesis.

\subsubsection{Ack1 Structure and Regulation}

Full length Ack1 is a 1038 amino acid long, $140 \mathrm{kDa}$ protein containing a wide variety of domains allowing it to interact with an assortment of different proteins, and therefore can be involved in many cellular processes and/or subject to many forms of regulation. Beginning from the N-terminus, Ack1 contains a SAM domain, kinase domain, SH3 domain, CRIB domain, a clathrin interacting region, a WW domain, a MIG6 homology region involved in binding EGFR, 
and a ubiquitin association domain (UBA) (Mahajan \& Mahajan, 2014). Along with these, Ack1 also has multiple proline rich regions centralized in its C-terminus which are capable of interacting with a number of effector proteins as well.

Unlike well characterized NRTKs like Src and Abl, the possible autoinhibitory mechanisms and activation mechanism of Ack1 remain unclear. It has been suggested that the SH3 domain of Ack1 forms an autoinhibitory interaction with the MIG6 homology region, as mutations in the SH3 domain show cell adhesion independent Ack1 activation (Lin et al., 2012). Another potential mechanism of autoinhibitory regulation involves one of Ack1's PRDs, as seen as a result of a S445P mutation causing constitutive activation of Ack1 (Lin et al., 2012).

\subsubsection{Ack1 Effects on Cell Physiology Mediated Through Clathrin Binding}

Of particular interest to this thesis is the ability of Ack1 to bind clathrin, and how this imparts on Ack1 unique functions in regulation of EGFR signaling. Overexpressed Ack1 has been found to co-localize with CCPs at the membrane, on intracellular CCVs, and on endosomes (Grøvdal et al., 2008; Shen et al., 2011). It has also been found in multiple studies to cause aggregation of clathrin when highly overexpressed, both at the plasma membrane and intracellularly (Grøvdal et al., 2008; Prieto-Echagüe et al., 2010; Teo et al., 2001). This aggregation has a distinct phenotype from that of moderately transfected cells, and the overexpression of Ack1 that causes aggregation also led to a reduction of the rate of EGF internalization (Grøvdal et al., 2008) as well as the rate of TfR endocytosis (Teo et al., 2001; Yang et al., 2001), consistent with broad impairment of CME. One potential explanation for these effects is that Ack1 shares similarity in its clathrin binding site with AP2. 
Immunoprecipitation of CHC pulls down less AP2 in conditions of Ack1 overexpression than in control cells (Yang et al., 2001), demonstrating the possibility that these two proteins compete for binding the same site, and overexpression of Ack1 can block AP2 interaction with clathrin, broadly affecting endocytic processes. This same binding site on clathrin is shared by a variety of other clathrin interacting proteins as well, adding to the complexity of signaling from CCPs at the plasma membrane. These studies show that Ack1 is intimately associated with clathrin and may be recruited to CCPs and participate in EGFR signaling, although the role of Ack1 in regulation of PI3K-Akt signaling in a possible clathrin-dependent manner remains to be examined.

\subsubsection{Aberrant Ack1 Associated with Cancer}

Recent data implies that Ack1 is involved in different types and stages of cancer. Amplification of the Ack1 gene correlates with metastasis and poor prognosis in lung and prostate cancer, and overexpression has been shown to increase invasiveness of cancer cells (Mahajan \& Mahajan, 2014). In addition to this, four identified missense mutations in the Ack1 sequence have been found to increase phosphorylation of key activation residue Y284 by up to 3-fold, as well as increase the overall kinase activity of Ack1 by 4-fold (Prieto-Echagüe et al., 2010). This finding is significant because the kinase activity of Ack1 has been shown to be required for anchorage independent growth and migration (Prieto-Echagüe et al., 2010). Therefore, there is an increased risk of tumor metastasis in tumors that contain cells expressing mutants of the NRTK as well as in those overexpressing WT Ack1. Ack1 is also of particular interest in studying EGFR as knockdown of Ack1 reduces cell surface expression of EGFR 
resulting in slower cell migration rates (Howlin et al., 2008). The mechanism by which Ack1 mediates these effects remains unclear, but it has been shown that p130Cas/BCAR1 (Modzelewska et al., 2006; Howlin et al., 2008), a well-known driver of cancer progression, acts downstream of Ack1 to mediate these effects. Thus, Ack is a very interesting potential candidate that may mediate clathrin-dependent signaling by controlling some aspects of EGFR signaling.

\subsubsection{Src Family Kinases}

The Src Family Kinases are the largest group of NRTKs in the cell (Gocek et al., 2014; Malarkey et al., 1995) . Their discovery played a critical role in early cancer research, as they were the first proto-oncogenes discovered which aided scientists in understanding that viruses carried mutated versions of normal cellular proteins (Parsons \& Parsons, 2004). There are 9 different genes encoding SFKs in humans: Src, Lck, Hck, Fyn, Yes, Fgr, Blk, Lyn, and Frk. While Src, Yes, and Fyn have been found to be ubiquitously expressed in all somatic cells, the other family members have restricted cell types in which they are found (in particular hematopoetic), as well as distinct sub-cellular localizations to which they are restricted (Abram \& Courtneidge, 2000). Given that the focus of my thesis will be on study of epithelial cells and in some cases epithelial-derived cancer cells, I will focus from henceforth on the ubiquitouslyexpressed Src, Yes and Fyn which are the major SFKs expressed in these cell types.

For some cellular functions, there is a high degree of functional redundancy between main members of the SFKs, as demonstrated by knockout mice experiments. Src ${ }^{-/}$knockout mice show very little change in phenotype, other than a minor osteoclast defect (Soriano et al., 1991), which is surprising considering the important role that the kinase plays intracellularly. For 
many cellular functions, it is only when two or more of the SFKs are knocked out in combination that there are visible changes in phenotype and cellular behaviours like growth and proliferation. Further studies in which Src/Fyn and Src/Yes double mutants were created resulted in perinatal death of the mice (Stein et al., 1994). Despite this redundancy seen in whole-body animal knockout studies, the functional redundancy of SFKs is not universal and specific roles of SKFs in specific cells and tissues have been reported (Gocek et al., 2014). For example, disruption of Lck alone in hematopoietic cells blocks T cell development altogether (Molina et al., 1992). Although studies like this using knockout mice show that various SFKs can compensate for loss of a specific SFK in the context of overall development, it remains unclear how redundant SFKs are at the molecular level. Additional specific examples of non-redundant functions of the SFK Fyn are discussed below.

Overall, SFKs share a highly conserved sequence and architecture with the exception of the first 50 amino acids in their Src Homology 4 (SH4) or "unique" domain. Preceding this is an N-terminal attachment site for saturated fatty acid addition (Gottlieb-Abraham et al., 2016). All SFKs are modified with the 14-carbon saturated fatty acid myristate (myristoylation) which is co-translationally and covalently attached to Glycine within a Met-Gly-Cys consensus sequence, mediated by N-Myristoyl Transferase (NMT) immediately following the translational start methionine residue (Ducker, 2005; Van't Hof \& Resh, 2000). Myristoylation allows for membrane targeting of SFKs by insertion of the lipid moiety into the inner leaflet of a membrane bilayer. In addition to myristoylation, all SFKs except for c-Src and Blk are palmitoylated. The 16-carbon saturated fatty acid palmitate is attached to cysteine 3 in most SFKs, and at either cysteine 5 (Lck) or cysteine 6 (Fyn) (Koegl et al., 1994; Paige et al., 1993; I. Sato et al., 2009). In 
addition to the membrane targeting by mysritoylation, the addition of palmitate specifically allows for SFK targeting to lipid rafts, discussed in further detail below.

\subsubsection{Unique cellular functions and localization of Src, Yes and Fyn.}

While c-Src and Yes are closely related homologs of the ubiquitously expressed SFKs, Fyn is more distantly related and therefore may be regulated differently (Hughes, 1996). Fyn has functions in somatic cells independently of Src and Yes. Specifically, Fyn is responsible for association with and phosphorylation of Focal Adhesion Kinase (FAK) within lipid rafts, which co-ordinates actin fiber and focal adhesion formation when bound to cell surface integrins, ultimately controlling cell shape and motility (Baillat et al., 2008; Saito et al., 2010). When coimmunoprecipitated in the presence or absence of SFK inhibitor PP2, Fyn but not c-Src or Yes, interacted with FAK in lipid raft fractions, and this interaction was not disturbed by treatment with PP2 (Baillat et al., 2008). Additionally, in $\mathrm{Src}^{(-/-)}, \mathrm{Yes}^{(--)}$and $\mathrm{Fyn}^{(-/-)}$fibroblasts, expression of Fyn restores FAK tyrosine phosphorylation on Y861, and expression of palmitoyl-modified mutants of Yes or Src which mimic palmitoylation of Fyn mirrors this effect on FAK phosphorylation (Sandilands et al., 2007). Recently Fyn has been found to directly phosphorylate Tau, a microtubule associated protein present abundantly in neurons and other cells of the central nervous system that when hyperphosphorylated collapses into aggregates, a hallmark of Alzheimer's disease (Iqbal et al., 2010; Nygaard, 2018). Studies using the SFK inhibitors Masitinib and Saracatinib have shown promising results in reversing Alzheimers disease symptoms to date, as a 50\% reduction in Fyn activity by sarcatinib treatment is sufficient to reduce insoluble phosphorylated tau and to reverse AD-like phenotypes (Kaufman et al., 2015). The initial signaling events proximal to the activated T-cell Receptor are also heavily dependent 
on Fyn activity specifically, and not any other SFKs (Palacios \& Weiss, 2004).

Since Src, Fyn and Yes are differentially required for biological processes in the cell, it is possible that their distinctiveness may come, at least in part, from differences in their spatial regulation and membrane targeting. Utilizing cells derived from Src-Yes-Fyn-deficient mice $\left(\mathrm{SYF}^{-/}\right)$(Klinghoffer et al., 1999), when individual SFKs were expressed they exhibited largely distinct cellular localizations. The vast majority of Src is retained in the perinuclear region in an inactive state in unstimulated cells, and upon PDGF stimulation, a proportion is targeted to membrane ruffles where it is active (Sandilands et al., 2007). Additionally, Fyn is found uniformly at the cell periphery in both stimulated and unstimulated cells, and Yes is found in discrete membrane patches containing the active form of the kinase (Sandilands et al., 2007). Fyn has also been described to reside within lipid rafts at the plasma membrane due to its dual fatty acylation, and to regulate signaling from here.

Another convincing piece of evidence that Fyn is regulated separately from other SFKs comes when looking at up-regulation of the kinases in cancer. While there is no significant difference in c-Src expression between normal epithelial and prostate cells compared to their cancerous counterparts, there is a 2.1-fold increase of Fyn expression in cancer cells relative to normal cells (Saito et al., 2010). This is also true for signaling partners of Fyn, including FAK and Paxillin (Saito et al., 2010). While there is much redundancy in some of the functions of SFKs, it is apparent that some functions of SKFs are kinase-specific as there are several examples of Fyn-specific functions in which c-Src and Yes are not able to compensate. 


\subsubsection{Fyn Structure and Regulation}

Fyn is a 536 amino acid long, $59 \mathrm{kDa}$ protein consisting of four main domains. At the Nterminus, there is an SH4 domain containing an N-terminal Met-Gly-Cys consensus sequence that mediates fatty acid acylation (Gottlieb-Abraham et al., 2016; Resh, 1994). As mentioned earlier and true for all SFKs, myristoylation allows for membrane targeting by insertion of the lipid moiety into the inner leaflet of a membrane bilayer. Although necessary, myristoylation is not sufficient for membrane anchorage of SFKs as they require a second signal in the form of palmitoylation (Gottlieb-Abraham et al., 2016). The addition of palmitate to Fyn specifically (of the ubiquitous Src, Yes, Fyn in somatic cells) allows for Fyn targeting to the plasma membrane, and specifically into lipid rafts (Filipp et al., 2003; Goulden et al., 2019; X. Liang et al., 2001; Wolven et al., 1997). Plasma membrane association of Fyn is more stable than Src due to this additional lipid modification, and Fyn mutants lacking their palmitoylation site (Fyn-C3S/C6S) are shifted in their membrane distribution from detergent-resistant microdomains to nondetergent resistant microdomains, where Src is found normally (Gottlieb-Abraham et al., 2016; Oneyama et al., 2009). Indeed, we see that in unstimulated $\mathrm{CD}^{+}{ }^{\mathrm{T}}$ cells, $>98 \%$ of Fyn is found localized to lipid rafts (Filipp et al., 2003). It is here in detergent-resistant microdomains that Fyn is required for T-cell receptor signaling (Cooke et al., 1991; Filipp \& Julius, 2004; Palacios \& Weiss, 2004). Given its near ubiquitous membrane association, Fyn localization may also be determined by membrane traffic and not through diffusion in the cytosol (Sato et al., 2009; Wolven et al., 1997), however this remains poorly understood.

Dysregulation of Fyn in cells has been shown to enhance cancer progression, therefore its activation is highly regulated. In the inactive state, Y527 in the C-terminal autoinhibitory region 
is phosphorylated and interacts with the kinase's own $\mathrm{SH} 2$ domain, an intramolecular interaction that induces a conformation that sequesters the ATP binding site and activation loop of the kinase domain (Parsons \& Parsons, 2004) (Figure 1.4). In this conformation, the SH3 domain also forms intramolecular interactions with the linker region located between the $\mathrm{SH} 2$ and the kinase domain. This inactivate state is promoted by the SFK negative regulator C-terminal Src kinase (Csk), responsible for phosphorylating Y527 (Thomas et al., 1991). Through either dephosphorylation of Y527 by the phosphatase CD45 (Burns et al., 1994), or interaction of the SH2 / SH3 domains with competing target proteins, the intrasteric inhibitory interactions are relieved, opening the conformation again. In order to achieve full activation and kinase activity, autophosphorylation at Y416 within the activation loop is required (Roskoski, 2004) (Figure 1.4). In this way, there is a balance between stimulatory and inhibitory phosphorylation events that control activity of Fyn. Given my focus on studying how clathrin and clathrin-associated proteins are required for regulation of EGFR signaling, studying specific clathrin-associated signaling adaptors that may bind and activate Fyn (or other SFKs) within these structures is of interest. One specific such protein that both binds clathrin and can associate with and activate Fyn is Target of Myb-like protein 1 (TOM1L1), as I discuss next.

\subsubsection{TOM1L1 and Fyn}

TOM1L1, also known as Src activating and signaling molecule (Srcasm), is a member of the TOM1 family of Vps27, Hrs and STAM (VHS) domain containing proteins comprised of TOM1, TOM1L1, AND TOM1L2. These proteins share N-terminal homology, including VHS and GGA and TOM1 (GAT) domains involved in vesicular trafficking and ubiquitin binding 
respectively (Liu et al., 2009). TOM1L1 is able to interact with a variety of effectors involved in the PI3K/Akt pathway via unique motifs within its C-terminus. These interactions include the SFKs Fyn, c-Src, and Yes (Li et al., 2005; Seykora et al., 2002), Grb2, the p85 regulatory subunit of PI3K (Seykora et al., 2002), and clathrin (Collin et al., 2007). These characteristics make TOM1L1 an attractive candidate protein for transduction of SFK signaling from the plasma membrane, and satisfy the requirements of a clathrin-binding effector protein.

Of particular interest to my work is the ability of TOM1L1 to bind and regulate the SFK Fyn. When phosphorylated at Y460, TOM1L1 acts as an activator of Fyn, increasing phosphorylation of Fyn 3 times compared to basal, a phenotype that is abolished in Y460F mutants of TOM1L1 (Seykora et al., 2002). Fyn's SH2 domain binds to pY460 on TOM1L1 and its $\mathrm{SH} 3$ domain associates with an $\mathrm{RLP}_{421}$ PLP motif, abolishing intramolecular interactions that would otherwise limit Fyn kinase activity (Li et al., 2005). Fyn has the ability to phosphorylate TOM1L1 on Y460 as well as other residues, and this phosphorylation by Fyn is specific to TOM1L1, as Fyn cannot phosphorylate TOM1 or TOM1L2 (Liu et al., 2009). The phosphorylation of Y460 on TOM1L1 is essential to mediate its interaction with Fyn, such that disruption of this site (e.g. by mutation), abolishes interaction of TOM1L1 with Fyn (Seykora et al., 2002). In addition, phosphorylation of TOM1L1 is necessary for its interaction with Fyn, the p85 regulatory subunit of PI3K, and Grb2 (Seykora et al., 2002), however it is not required for clathrin binding (Collin et al., 2007).

TOM1L1 interacts with clathrin directly using two distinct motifs found within TOM1L1's C-terminal region. The first is a leucine rich motif found at ${ }^{401}$ LQPVSL which resembles the canonical clathrin binding box in many other proteins that bind clathrin at the Nterminal domain of CHC (Collin et al., 2007). The second motif TOM1L1 uses to bind clathrin is 
${ }^{447}$ FDPL, a novel sequence that interacts with the C-terminus of CHC (Liu et al., 2009), important as this means TOM1L1 does not compete for binding clathrin with other clathrin endocytic accessory proteins that interact with the N-terminal protein interaction hub. This also suggests that artifacts of overexpression of TOM1L1 may occur to a lesser extent compared to other endocytic accessory proteins, as it will not compete for interaction with clathrin with other proteins and potentially exclude important endocytic regulators from CCPs.

\subsubsection{TOM1L1 is an Important Clathrin-Binding Signaling Regulator}

TOM1L1 becomes tyrosine phosphorylated in the presence of EGF, TGF- $\alpha$ (Li et al., 2005), PDGF, and fibroblast growth factor (FGF) (Liu et al., 2009). Previous work from the Antonescu lab suggests that TOM1L1 may be necessary for signaling events downstream of EGFR, as siRNA gene silencing of TOM1L1 decreases Gab1 and Akt phosphorylation following EGF stimulation (Judge, 2015). In addition, overexpression of TOM1L1 enhances activation of SFKs Fyn and Src (but not Yes), as well as enhances activation of the MAPK signaling pathway in the absence of EGF (Li et al., 2005). Additionally, TOM1L1 is important for endocytosis and degradation of EGFR (Liu et al., 2009). TOM1L1 is largely found within the cytoplasm in the basal state, but following EGF stimulation in cells, endogenous TOM1L1 is recruited to the plasma membrane (Liu et al., 2009).

In addition to recruitment of TOM1L1 to the plasma membrane where it may have roles in regulating signaling, TOM1L1 may also have a contribution to regulation of signals from intracellular compartments. Following CME, once a CCV is internalized it fuses with the early endosome approximately 10-20 minutes following initial ligand stimulation. TOM1L1 is found 
to localize to early endosomes also following this time line, as it co-localizes with the Early Endosome Antigen 1 (EEA1) puncta (Liu et al., 2009). Expression of TOM1L1 harboring mutations in its binding sites to Fyn or Grb2 results in a decreased rate of endocytosis of EGFR as measured by EGF internalization from the plasma membrane. Gene silencing of TOM1L1 by siRNA displays the same phenotype, and is not able to be rescued by transfection with TOM1L1 clathrin binding mutants (Liu et al., 2009). Importantly, TOM1L1 is also found to be overexpressed in many types of cancers. Taken together, this work suggests that TOM1L1 may be acting as a link between Fyn and CCPs, effectively bringing the kinase in proximity of EGFR and other signaling intermediates allowing phosphorylation and signal propagation. This aspect of TOM1L1 function has not yet been examined, and will be examined in this thesis. 


\subsection{Rationale and Hypothesis}

The ligand-dependent internalization of many RTKs at the plasma membrane represents a principal process regulating the duration and propagation of cell signaling, preventing overstimulation that could potentially lead to cellular transformation. Indeed, amplification, mutation, and overexpression of EGFR, which has key roles in cellular physiology, metabolism and survival, is a well-known driver of many forms of human cancers, most notably of the lung, breast, and colorectal subtypes (Roskoski, 2014). Given that disruptions in EGFR are a wellknown driver of tumor progression and cancer cell survival, it is targeted in many anticancer therapies to date. It is therefore important that we delineate the regulation of cellular signaling downstream of EGFR to identify potential new drug targets for cancer research.

Cell surface availability of EGFR is regulated by CME, and signaling downstream of EGFR is known to occur from endosomes (Murphy et al., 2009; Stasyk et al., 2007; Wang et al., 2002). However, several recent lines of evidence suggest that EGFR may instead signal primarily from the plasma membrane (Ibach et al., 2015; Offterdinger et al., 2008; RosselliMurai et al., 2018; Sousa et al., 2012). Our lab has demonstrated that pharmacological perturbation of clathrin with Pitstop 2, but not inhibition of receptor endocytosis by inhibition of Dynamin 2, impairs EGF-stimulated Akt activation (Garay et al., 2015). Moreover, we have found that some key EGFR signaling intermediates such as phosphorylated Gab1 are enriched with a subset of CCPs following EGF stimulation (Garay et al., 2015). We thus propose that in addition to their well-known function as endocytic portals, some CCPs have a direct role in controlling EGFR signaling at the plasma membrane prior to receptor internalization. How CCPs and clathrin may directly control EGFR signaling at the plasma membrane prior to receptor endocytosis had remained poorly understood, which I have now examined here. 
I therefore hypothesize that CCPs have a direct role in controlling EGFR signaling at the plasma membrane prior to receptor internalization, and that the selective recruitment of certain signaling proteins to a subset of CCPs containing EGFR imparts upon these structures the properties of signaling nanodomains required to control EGFR signaling.

To test this hypothesis, I will undertake the following three main objectives:

1. To determine the capacity in which clathrin is required for receptor signaling at the plasma membrane, which I examine in Chapter 3

2. To determine the role of non-receptor tyrosine kinases in EGFR signaling, which I examine in Chapter 4

3. To determine the spatial organization and regulation of EGFR-positive signaling nanodomains at the plasma membrane, which I examine in Chapter 5 


\subsection{Chapter 1 Figures}

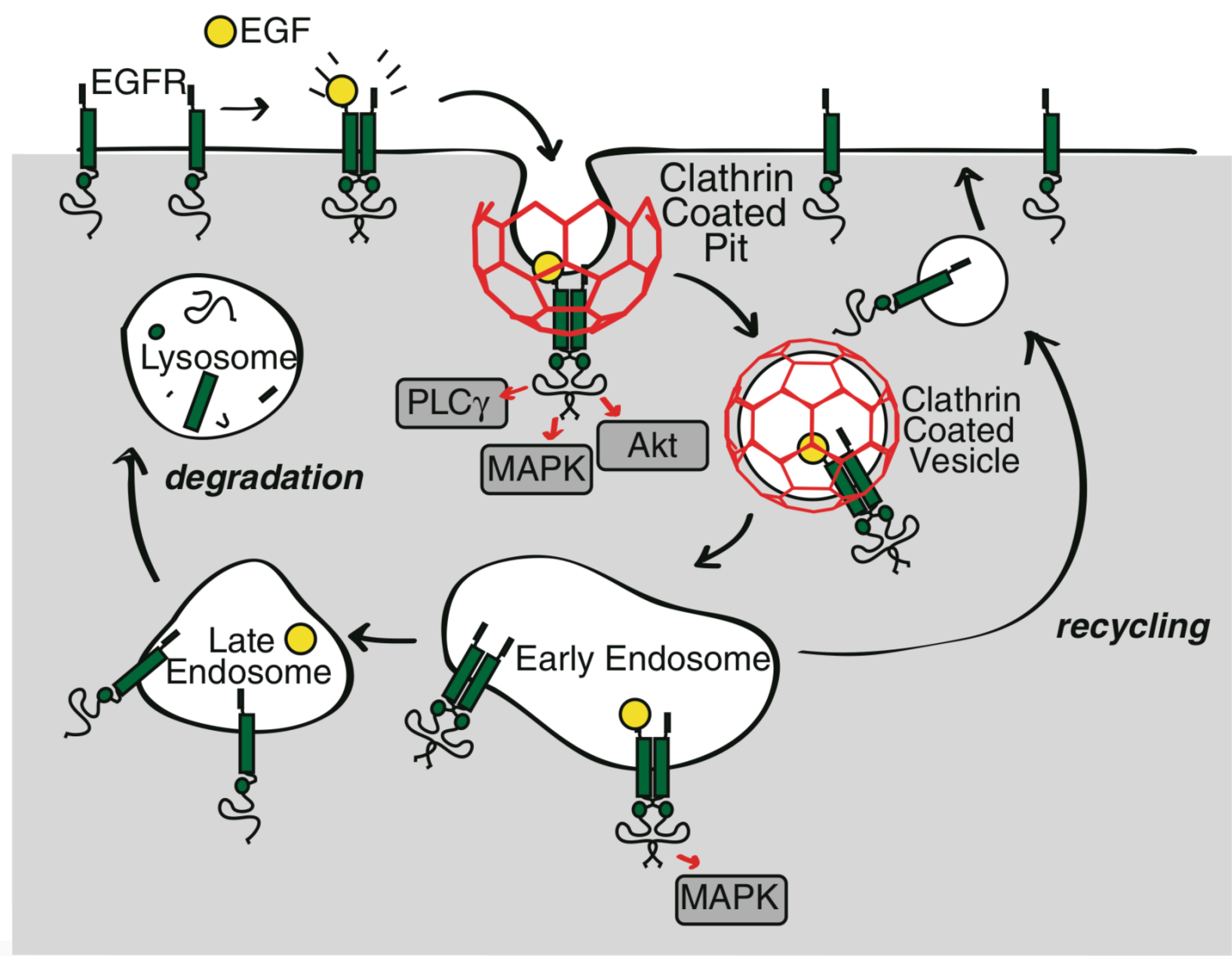

Figure 1.1 EGF stimulation regulates EGFR membrane traffic and signaling.

EGF binding to EGFR leads to the formation of asymmetric receptor dimers and triggers the activation of the EGFR kinase domain. As a result, ligand-bound EGFR at the plasma membrane elicits the activation of several signaling pathways including those leading to the activation of mitogen-activated protein kinase (MAPK), phospholipase $\mathrm{C} \gamma 1$ (PLC $\gamma 1$ ), and Akt, as well as recruitment to clathrin-coated pits (CCPs), eventually leading to receptor endocytosis. Following internalization, EGFR resident within clathrin-coated vesicles is routed to early endosomes, from which it may transit to late endosomes and lysosomes (leading to EGFR degradation and signal termination), undergo recycling (leading to continued EGFR signaling), or route to other cellular compartments such as the nucleus (not shown). The activation of specific EGFR signals 
can occur at the plasma membrane or in various endosomal compartments. (Lucarelli et al., 2017) 


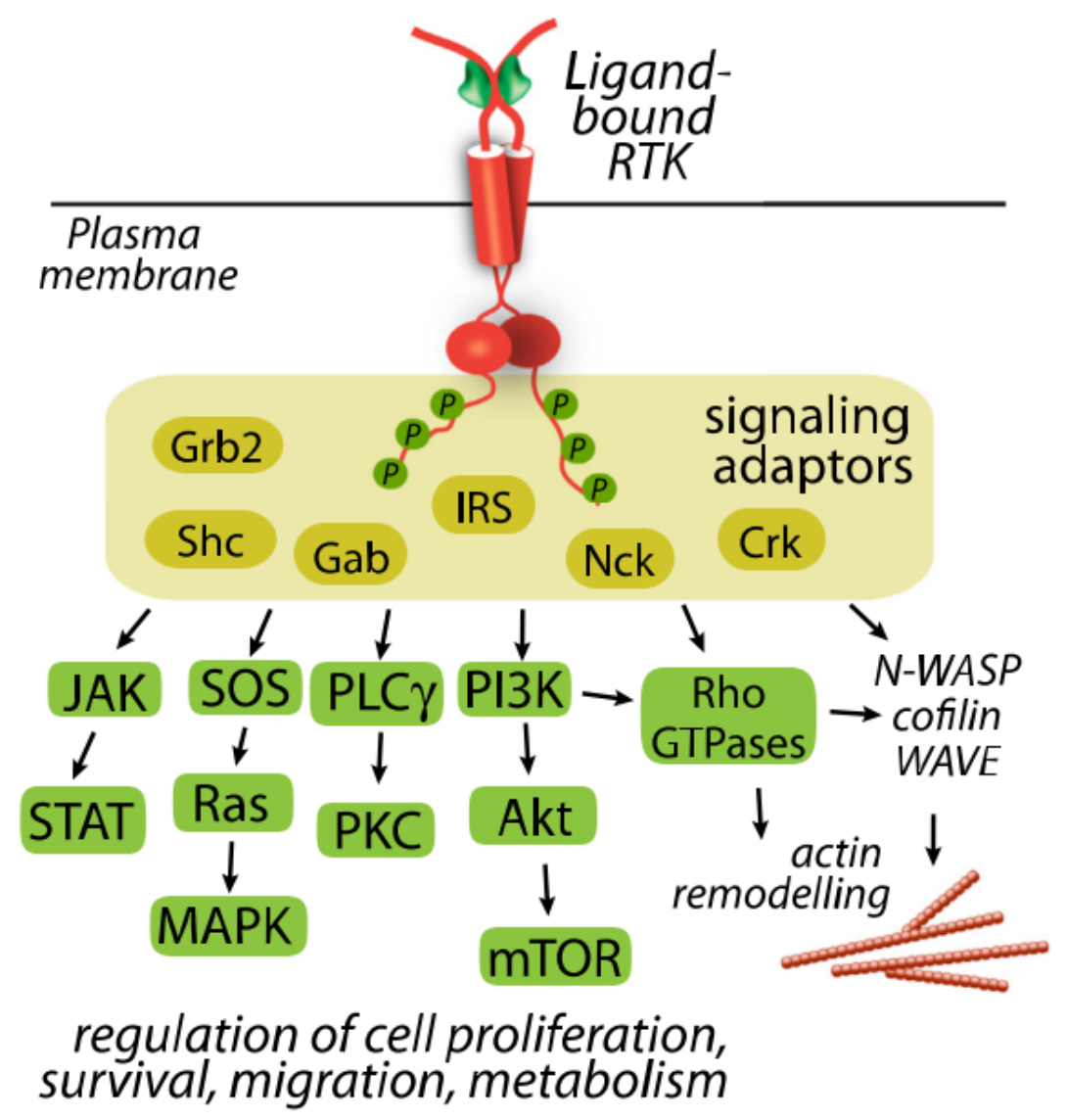

Figure 1.2 Signaling pathways downstream of EGFR activation.

Following ligand binding, EGFR phosphorylation results in the activation of numerous signaling cascades within the cell that regulate cell proliferation, survival, migration, and metabolism. Shown above are the major signaling cascades downstream of EGF stimulation of EGFR, which include (from left to right) the JAK/STAT, MAPK, PLC $\gamma$, PI3K/Akt, and actin remodeling pathways. 


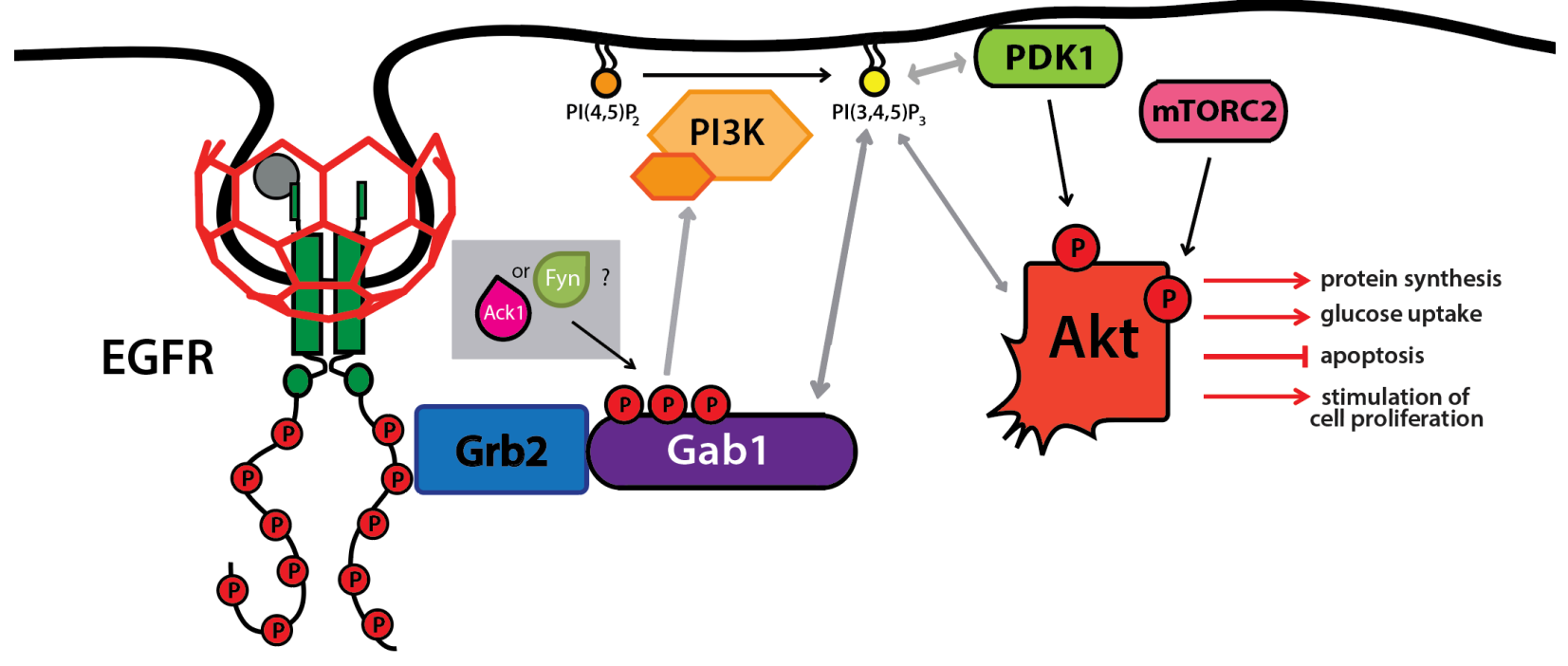

Figure 1.3 EGFR activation of the PI3K/Akt pathway.

EGF stimulation leads to phosphorylation of multiple tyrosine residues within the C-terminal tails, and recruitment of secondary signaling messengers Grb2 and Gab1. Gab1 becomes phosphorylated at multiple residues, however the mechanism by which this occurs remains unclear. Gab1 phosphorylation activates PI3K, leading to the production of $\mathrm{PIP}_{3}$ from $\mathrm{PIP}_{2}$ at the plasma membrane, and the activation of Akt downstream. 

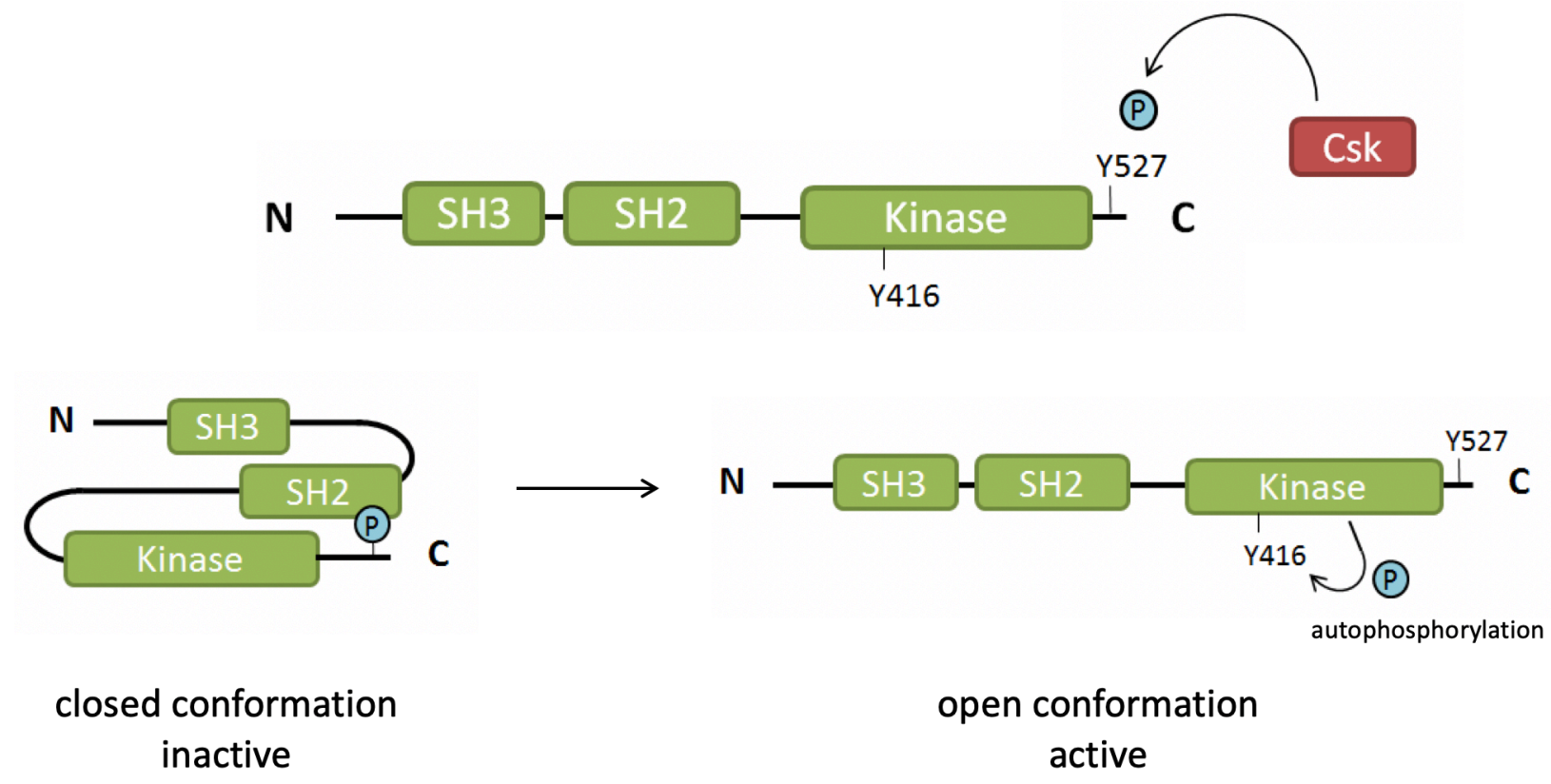

Figure 1.4 Mechanism of activation of Src Family Kinases.

In the inactive state, Y527 in the C-terminal autoinhibitory region is phosphorylated by Cterminal Src Kinase (Csk) and interacts with the kinase's own SH2 domain, an intramolecular interaction that induces a conformation that sequesters the ATP binding site and activation loop of the kinase domain. In this conformation, the SH3 domain also forms intramolecular interactions with the linker region located between the SH2 and the kinase domain. Through either dephosphorylation or interaction of the SH2 / SH3 domains with competing target proteins, the intrasteric inhibitory interactions are relieved, opening the conformation. In order to achieve full activation and kinase activity, autophosphorylation at Y416 within the activation loop is required. 
Table 1. Proteins that associate with clathrin and/or regulate Clathrin Mediated Endocytosis

\begin{tabular}{|c|c|c|c|}
\hline $\begin{array}{c}\text { Gene } \\
\text { Name }\end{array}$ & Protein Name & $\begin{array}{c}\text { Function in Clathrin Mediated } \\
\text { Endocytosis }\end{array}$ & Reference \\
\hline SNAP91 & AP180 & $\begin{array}{l}\text { Main structural protein of CCPs, stimulates } \\
\text { assembly of clathrin lattices at the plasma } \\
\text { membrane with AP2 and induces curvature }\end{array}$ & $\begin{array}{l}\text { (Res, Prasad, \& } \\
\text { Lippoldt, 1988) }\end{array}$ \\
\hline LCLAT1 & LYCAT & $\begin{array}{l}\text { Converts lysophosphatidic acid (LPA) into } \\
\text { phosphatidic acid (PA) and determines fatty } \\
\text { acids }\end{array}$ & $\begin{array}{l}\text { (Bone et al., } \\
\text { 2017; Imae et } \\
\text { al., 2012) }\end{array}$ \\
\hline MBOAT7 & $\begin{array}{l}\text { Lysophospholipid } \\
\text { Acyltransferase } 7\end{array}$ & $\begin{array}{l}\text { Converts lysophosphatidylinositol into } \\
\text { phosphatidylinositol (PI) }\end{array}$ & $\begin{array}{c}\text { (Anderson et al., } \\
\text { 2013) }\end{array}$ \\
\hline EPN1 & Epsin & $\begin{array}{l}\text { Binds } \mathrm{PIP}_{2} \text { and adds bending stress to the } \\
\mathrm{PM} \text {, inducing membrane curvature for CCPs }\end{array}$ & $\begin{array}{l}\text { (Chen et al., } \\
\text { 1998; Horvath } \\
\text { et al., 2007) }\end{array}$ \\
\hline PICALM & $\begin{array}{l}\text { Phosphatidylinositol } \\
\text { Binding Clathrin } \\
\text { Assembly Protein }\end{array}$ & $\begin{array}{l}\text { Recruits clathrin and AP2 to the PM, } \\
\text { regulates size of CCPs }\end{array}$ & $\begin{array}{l}\text { (Tebar, } \\
\text { Bohlander, \& } \\
\text { Sorkin, 1999) }\end{array}$ \\
\hline HIP1 & $\begin{array}{l}\text { Huntington } \\
\text { Interacting Protein }\end{array}$ & Coordinates actin remodeling during $\mathrm{CME}$ & $\begin{array}{l}\text { (Mishra et al., } \\
\text { 2001) }\end{array}$ \\
\hline SNX9 & Sorting Nexin 9 & $\begin{array}{l}\text { Suggested to regulate dynamin recruitment to } \\
\text { CCPs; binds } \mathrm{PIP}_{2} \text { rich membranes }\end{array}$ & $\begin{array}{l}\text { (reviewed in } \\
\text { Mousavi et al., } \\
\text { 2004) }\end{array}$ \\
\hline AMPH & Amphiphysin & $\begin{array}{l}\text { Dynamin recruitment to CCPs; BAR domain } \\
\text { containing and } \mathrm{m}\end{array}$ & $\begin{array}{l}\text { (McMahon, } \\
\text { Wigge, \& } \\
\text { Smith, 1997) }\end{array}$ \\
\hline ARRB1 & B-Arrestin & $\begin{array}{l}\text { Binds clathrin and AP2; cargo selection for } \\
\text { GPCRs }\end{array}$ & $\begin{array}{c}\text { (Zhang et al., } \\
1996)\end{array}$ \\
\hline DAB2 & Disabled Homolog 2 & $\begin{array}{l}\text { Cargo selection adaptor protein for LDLR } \\
\text { and integrins; also binds AP2 and } \mathrm{PIP}_{2}\end{array}$ & $\begin{array}{l}\text { (reviewed in } \\
\text { Mousavi et al., } \\
\text { 2004) }\end{array}$ \\
\hline SH3KBP1 & CIN85 & $\begin{array}{l}\text { Binds } \mathrm{Cbl} \text { and induces ubiquitination and } \\
\text { downregulation of EGFR and MET receptors }\end{array}$ & $\begin{array}{l}\text { (Take et al., } \\
\text { 2000) }\end{array}$ \\
\hline $\begin{array}{l}\text { SYNJ1 } \\
\text { SYNJ2 }\end{array}$ & $\begin{array}{l}\text { Synaptojanin-1 } \\
\text { Synaptojanin-2 }\end{array}$ & $\begin{array}{l}\text { Phosphoinositide phosphatases that are } \\
\text { involved in CCP formation (1) and } \\
\text { membrane trafficking (2) }\end{array}$ & $\begin{array}{l}\text { (reviewed in } \\
\text { Mousavi et al., } \\
\text { 2004) }\end{array}$ \\
\hline FCHO1 & $\begin{array}{l}\text { F-Bar Domain Only } \\
\text { Protein } 1\end{array}$ & Membrane binding nucleator of early CCPs & $\begin{array}{l}\text { (Henne et al., } \\
\text { 2010) }\end{array}$ \\
\hline DNAJC6 & Auxillin & Involved in $\mathrm{CCV}$ uncoating & $\begin{array}{l}\text { (E. Ungewickell } \\
\text { et al., 1995) }\end{array}$ \\
\hline AAK1 & $\begin{array}{l}\text { AP2-associated } \\
\text { protein kinase } 1\end{array}$ & $\begin{array}{l}\text { Ser/Thr kinase that phosphorylates AP } 2 \\
\text { promotes binding to RTKs and other cargo }\end{array}$ & $\begin{array}{c}\text { (Conner \& } \\
\text { Schmid, 2002) }\end{array}$ \\
\hline TNK2 & Ack1 & $\begin{array}{l}\text { Binds clathrin and activated EGFR; involved } \\
\text { in EGFR degradation and signaling }\end{array}$ & $\begin{array}{l}\text { (W Yang et al., } \\
\text { 2001) }\end{array}$ \\
\hline
\end{tabular}




\begin{tabular}{|c|c|c|c|}
\hline SCYL2 & CVAK104 & Ser/Thr kinase associated with CCVs & $\begin{array}{c}\text { (Conner \& } \\
\text { Schmid, 2005) }\end{array}$ \\
\hline MLK2 & MAP3K10 & $\begin{array}{l}\text { Influences CCV trafficking and signals the } \\
\text { JUN pathway }\end{array}$ & $\begin{array}{l}\text { (Akbarzadeh et } \\
\text { al., 2002) }\end{array}$ \\
\hline TOM1L1 & TOM1-like Protein 1 & $\begin{array}{l}\text { Adaptor protein that binds clathrin; promotes } \\
\text { Fyn activation }\end{array}$ & $\begin{array}{l}\text { (Collin et al., } \\
\text { 2007) }\end{array}$ \\
\hline PXN & Paxillin & $\begin{array}{l}\text { Signal transducing adaptor protein located at } \\
\text { focal adhesions, binds to } \beta \text {-integrin }\end{array}$ & $\begin{array}{l}\text { (reviewed in } \\
\text { Mousavi et al., } \\
\text { 2004) }\end{array}$ \\
\hline ITSN1 & Intersectin-1 & $\begin{array}{l}\text { GEF for Cdc } 42 \text {; provides a link between } \\
\mathrm{CME} \text { and actin assembly at CCPs }\end{array}$ & $\begin{array}{l}\text { (Martin et al., } \\
\text { 2006) }\end{array}$ \\
\hline
\end{tabular}




\section{Chapter 2. Materials and Methods}

\subsection{Materials and Antibodies}

DMEM/F12, fetal bovine serum (FBS), penicillin/streptomycin solution, and sterile HEPES buffer were obtained from Life Technologies (Carlsbad, CA). Human EGF (unlabeled), EGF (complexed to Alexa Fluor 555 [A555-EGF] or rhodamine [rhodamine-EGF]), and Tfn (complexed to Alexa Fluor 555 [A555-Tfn] or Alexa Fluor 647 [A647-Tfn]) were obtained from Life Technologies (Carlsbad, CA). HGF was obtained from Life Technologies (Carlsbad, CA). Superblock blocking buffer was obtained from Thermo Fisher (Rockford, IL).

Antibodies recognizing specific proteins were used as follows: anti-phospho-EGFR (pY1068), anti-phospho-Gab1 (pY307, 627), anti-phospho-Akt (pT308), anti-phospho-Src (Y416), anti-phospho-p44/42 MAPK, Fyn, Akt, Gab1, epsin, and endophilin antibodies were obtained from Cell Signaling Technology (Danvers, MA); anti-phospho-Akt (pS473) antibody was obtained from Life Technologies (Carlsbad, CA); AP2 (AP6) was obtained from Abcam (Cambridge, MA); amphiphysin was obtained from Synaptic Systems (Goettingen, Germany); anti-actin and anti-clathrin heavy chain (TD.1) used for immunoblotting were from Santa Cruz Biotechnology (Santa Cruz, CA). Fluorophore-conjugated secondary antibodies were from Jackson ImmunoResearch (West Grove, PA). Horseradish peroxidase (HRP)-conjugated secondary antibodies were obtained from Cell Signaling Technology (Danvers, MA). Rhodamine-EGF was generated in house. 


\subsection{Cell Culture and Cell Lines}

Wild-type human retinal pigment epithelial cells (ARPE-19; RPE cells herein), and ARPE- 19 cells stably expressing clathrin light chain fused to enhanced GFP (RPE-GFP-CLC) or fused to TagRFP-T (RPE-RFP-CLC) were previously described (Bone et al., 2017; Aguet et al., 2013; Delos Santos et al., 2017). Cells were cultured in DMEM/F12 (Life Technologies) supplemented with $10 \%$ fetal bovine serum (Life Technologies), $100 \mathrm{ug} / \mathrm{ml}$ penicillin, and 100 $\mu \mathrm{g} / \mathrm{ml}$ streptomycin (Life Technologies) at $37^{\circ} \mathrm{C}$ and $5 \% \mathrm{CO}_{2}$.

MCF10A cells were obtained from Dr. Vuk Stambolik's lab at the Princess Margaret Cancer Centre. Cells were cultured in DMEM/F12 (Life Technologies) supplemented with 10\% fetal bovine serum (Life Technologies), $20 \mathrm{ng} / \mathrm{ml} \mathrm{EGF} \mathrm{(Gibco),} 0.5 \mathrm{mg} / \mathrm{ml}$ hydrocortisone (Sigma Aldrich), $100 \mathrm{ng} / \mathrm{ml}$ cholera toxin, $10 \mathrm{ug} / \mathrm{ml}$ Insulin (Sigma Aldrich), $100 \mathrm{ug} / \mathrm{ml}$ penicillin, and $100 \mu \mathrm{g} / \mathrm{ml}$ streptomycin (Life Technologies) at $37^{\circ} \mathrm{C}$ and $5 \% \mathrm{CO}_{2}$.

\section{3 cDNA Constructs}

sfGFP-FYN-N-10 (sfGFP is superfolder GFP with enhanced stability and folding kinetics, derived from eGFP (Pédelacq et al., 2006); herein we refer to this construct as eGFPFyn) was a gift from Michael Davidson (Addgene plasmid \# 56294). eGFP-tagged TOM1L1 was generated by seamless cloning. Briefly, human TOM1L1 (pDNR-LIB, plasmid number 4612157, purchased from Dharmacon, Inc) was amplified by PCR using primers as follows ( $\left.5^{\prime}->3^{\prime}\right)$ : CGA GCT GTA CAA GGG ACT CAG ATC TAG ATC ACA GAT GGC GTT TGG and GCA GAA TTC GAA GCT CTA TTA TTT ACT TAA G, and eGFP-C1 (Clontech) was amplified using primers as follows(5'->3'): CCA AAC GCC ATC TGT GAT CTA GAT CTG AGT CCC TTG 
TAC AGC TCG and C TTA AGT AAA TAA TAG AGC TTC GAA TTC TGC. The resulting PCR products were mixed, subjected to digestion by Dpn1, and used to transform DH5 $\alpha$ competent E. coli cells, followed by selecting resistant colonies and verification of GFPTOM1L1 by sequencing. HA- or FLAG-tagged 21 WT, Fyn-binding defective (Y460F), and clathrin-binding defective (447FDPL450AAAA) TOM1L1 constructs were previously described (Liu et al., 2009b).

\subsection{Plasmid and siRNA Transfections}

cDNA transfections were performed using Lipofectamine 2000 (Life Technologies) as per manufacturer's instructions. Cells were washed in $2 \mathrm{X}$ warm PBS and media was replaced with Opti-MEM medium (Gibco, Life Technologies). Lipofectamine 2000 reagent ( $3 \mu \mathrm{L} /$ well) and cDNA $(1 \mu \mathrm{g} /$ well) were mixed separately in $50 \mu \mathrm{L}$ OptiMem, combined in a 1:1 ratio, vortexed, and incubated at room temperature for 10-15 min before pipetting the mixture dropwise onto plated cells in Opti-Mem. Experiments were performed 16- $20 \mathrm{~h}$ following transfection.

siRNA transfections were performed using custom-synthesized siRNAs using RNAiMAX transfection reagent (Life Technologies) as per manufacturer's instructions. Briefly, each siRNA was transfected at a concentration of $220 \mathrm{pmol} / 1$ with $3 \mathrm{uL} /$ well of transfection reagent in Opti-MEM Medium (Life Technologies). Cells were incubated with the siRNA complexes for $4 \mathrm{~h}$, after which cells were washed and replaced with regular growth medium. siRNA transfections were performed twice (72 and $48 \mathrm{~h}$ ) before each experiment. Sequences used were as follows (sense): control (nontargeting): CGU ACU GCU UGC GAU ACG GUU; 
Fyn: AGG AAG AGC UCU GAA AUU AUU; TOM1L1: GUG AGA AAC UGA AUG UAU

UUU; Epsin: ACU CAG AGG CGG AGA UCA AUU; Amphiphysin: GCG AGA ACU CCG AGG AUA UUU; Endophilin: UCG UCA AGC UCU AGA GAA AUU.

\subsection{Cell and Inhibitor Treatments}

All cells were serum deprived for $1 \mathrm{~h}$ before experimental assays unless otherwise stated. Cells were treated with Dasatinib (concentrations as indicated) or a corresponding volume of dimethyl sulfoxide (DMSO; vehicle control) for 20 min before stimulation with either $5 \mathrm{ng} / \mathrm{mL}$ or $20 \mathrm{ng} / \mathrm{mL}$ EGF (human; Life Technologies), or left unstimulated (basal) as indicated. All inhibitor treatments were performed subsequent to a $1 \mathrm{~h}$ serum deprivation.

\subsection{Whole-Cell Lysates and Western Blotting}

After transfection, treatment with inhibitors, and/or stimulation with EGF, whole-cell lysates were prepared in Laemmli sample buffer (LSB; 0.5 M Tris, pH 6.8, glycerol, 10\% SDS, $10 \% \beta$-mercaptoethanol, and 5\% bromophenol blue; all from BioShop, Burlington, Canada) supplemented with a protease and phosphatase inhibitor cocktail (1 $\mathrm{mM}$ sodium orthovanadate, $10 \mathrm{nM}$ okadaic acid, and $20 \mathrm{nM}$ Protease Inhibitor Cocktail, each obtained from BioShop). Lysates were then heated at $65^{\circ} \mathrm{C}$ for $15 \mathrm{~min}$ and passed through a 27.5 -gauge syringe 3 times. Proteins were resolved by glycine-Tris SDS-PAGE followed by transfer onto a polyvinylidene fluoride membrane; they were washed, blocked, and incubated with antibodies as previously described (Antonescu et al., 2011). Western blot signals to detect the intensity corresponding to 
phosphorylated proteins (e.g., pAkt) were obtained as previously described (Delos Santos et al., 2017) by signal integration in an area corresponding to the appropriate lane and band for each condition. This measurement is then normalized to the loading control (e.g., actin) signal, and subsequently normalized to the total Akt signal, obtained following reblotting. In each experiment, the resulting normalized pAkt/ total Akt signal in each condition was expressed as a fraction of the normalized pAkt/total Akt measurement in the control condition stimulated with EGF for $5 \mathrm{~min}$. Statistical analysis was performed by ANOVA followed by Tukey post-test, with $\mathrm{p}<0.05$ used as a threshold for establishing differences between experimental conditions.

Values shown in quantification of western blot experiments are normalized to a control condition to demonstrate variance of each condition relative to the control for which it is being compared to. This control condition that all values are normalized to represent the EGFstimulated condition of the least perturbed state of the cells (ie. control siRNA and/or vehicle control treated conditions) to best score the effect of the treatment relative to this condition, as has been previously done (Garay et al., 2015; Delos Santos et el., 2017; Lucarelli et al., 2015; Antonescu et al., 2011).

\subsection{Immunofluorescence Staining}

For detection of total cellular protein, after treatments as indicated cells were washed in ice cold PBS and fixed with 4\% paraformaldehyde for $20 \mathrm{~min}$ on ice, followed quenching of fixative in $100 \mathrm{mM}$ glycine for 20 mins, cell permeabilization in $100 \mathrm{mM}$ glycine $+0.1 \%$ Triton X-100 (all solutions made in PBS), and then blocking in Superblock Blocking Buffer (Thermo Fisher Scientific). Subsequently, cells were stained with primary and secondary antibodies for 1 hour each and 10 mins of washing in PBS between, and then mounted on glass slides in 
fluorescence mounting medium (Dako, Carpinteria, CA) or retained within aqueous medium (PBS) for imaging by TIRF microscopy.

Immunofluorescence staining of cell-surface EGFR was performed by first labeling intact cells at $4{ }^{\circ} \mathrm{C}$ with anti-EGFR antibodies, followed by fixation, quenching, and secondary antibody staining (Garay et al., 2015), using anti-EGFR (mAb108, collected in-house from a hybridoma cell line from the American Type Culture Collection, Manassas, VA). Briefly, following treatments, cells were rapidly washed with ice-cold PBS, and then incubated with primary antibody solution on ice, prior to cell permeabilization. Following washing of unbound primary antibodies, cells were then fixed and permeabilized, and stained with secondary antibodies as described above.

\subsection{Fluorescence Microscopy}

Wide-field epifluorescence microscopy experiments were performed on an Olympus IX83 Inverted Microscope with a 100x objective, coupled to a Hamamatsu ORCA-Flash4.0 digital camera (Olympus Canada, Richmond Hill, ON).

Total internal reflection fluorescence (TIRF) microscopy experiments were performed on a Quorum (Guelph, ON, Canada) Diskovery instrument, comprised of a Leica DMi8 microscope equipped with a $63 \mathrm{X} / 1.49 \mathrm{NA}$ TIRF objective with a $1.8 \mathrm{X}$ camera relay (total magnification 108X). Imaging was done using 405-, 488-, 561-, and 637-nm laser illumination and 450/55, 525/50, 620/60, and 700/75 emission filters and acquired using a Zyla 4.2Plus sCMOS camera (Hamamatsu). For live-cell imaging experiments, cells were maintained at constant $37^{\circ} \mathrm{C}$ during imaging, in phenol-free DMEM/F12 media (Gibco) supplemented with 20 mM HEPES and 20 
ng/mL EGF. Some TIRF experiments also involved acquisition of identical fields of view using widefield epifluorescence microscopy.

Structured illumination microscopy (SIM) was performed using a Zeiss Elyra PS.1 super resolution inverted microscope, as previously described (Bautista et al., 2018). Samples were imaged at an effective magnification of $101 x(63 x$ objective $+1.6 x$ optovar tube lens $)$ on an oil immersion objective. $488 \mathrm{~nm}, 561 \mathrm{~nm}$ and $643 \mathrm{~nm}$ laser lines were directed into the microscope optical train via a multimode fiber coupler. The lasers were passed through a diffraction grating, and a series of diffraction orders $(-1,0,+1)$ were projected onto the back focal plane of the objective. These wavefronts were collimated in the objective to create a three-dimensional sinusoidal illumination pattern on the sample. The diffraction grating was then rotated and translated throughout the acquisition to create patterned offset images containing encoded high spatial frequency information. Three lateral positions were acquired at each of five $\left(72^{\circ}\right)$ diffraction grating rotations for a total of 15 raw images. SIM imaging with all lasers was carried out at exposures varying from $50 \mathrm{~ms}$ to $250 \mathrm{~ms}$, with laser power varying between $3-10 \%$, and a gain level of $<100$. Imaging parameters were adjusted iteratively to achieve the best possible equalization of pixel intensity dynamic range across channels. Raw SIM image stacks were processed in Zen under the Structured Illumination toolbar. The noise filter for Wiener deconvolution was set to a value of $1.0 \times 10-4.5$ to maximize the recovery of high spatial frequency information while minimizing illumination pattern artifacts. Processed SIM images were then aligned via an affine transformation matrix of pre-defined values obtained using 100 nm multicolor Tetraspeck fluorescent microspheres (ThermoFisher Scientific).

\subsection{Analysis of Fixed-Cell Fluorescence}


Fluorescence intensity of Akt phosphorylation was determined following antibody labeling of phosphorylated Akt and widefield fluorescence microscopy was determined using ImageJ software (National Institutes of Health, Bethesda, MD) (Schneider et al., 2012) as previously described (Antonescu et al., 2008; Garay et al., 2015). Briefly, a cell outline was created manually, followed by measurement of the mean fluorescence intensity corresponding to pAkt in each cell. The non-specific signal, determined similarly in cells subjected to immunofluorescence labeling without primary antibody, was subtracted from all values. Cells transfected for various Fyn, Ack1, or TOM1L1 constructs were identified by significant fluorescence in the appropriate channel. Differences in the mean pAkt signal between different conditions were determined by one-way ANOVA with Tukey post-test, with a threshold of $p<$ 0.05 for statistically significant difference between conditions.

\subsubsection{CLS Detection and Quantification of Protein Fluorescence Intensity}

Systematic, unbiased detection and analysis of clathrin-labelled structures (CLSs) in fixed cells was done as previously described (Delos Santos et al., 2017; Lucarelli et al., 2017), using custom software developed in Matlab (Mathworks Corporation, Natick, MA), as described in (Aguet et al., 2013; Garay et al., 2015). Briefly, diffraction-limited clathrin structures were detected using a Gaussian-based model method to approximate the point-spread function of eGFP-CLCa, RFP-T-CLCa or AP2 puncta ('primary' channel). The TIRF intensity corresponding to various proteins in a 'secondary' (or 'tertiary') channel (eGFP-Fyn, eGFPTOM1L1, eGFP-Ack1, rhodamine-EGF, epsin, endophilin, or amphiphysin) within CLSs was determined by the amplitude of the Gaussian model for the appropriate fluorescence channel for 
each CLS structure detected in the 'primary' channel. As such, the measurements of fluorescently labelled proteins within CLSs represent their enrichment relative to the local background fluorescence in the immediate vicinity of the detected CLS. Similar measurements were done using widefield epifluorescence microscopy images (as the 'secondary' channel) following detection of CLSs in the corresponding TIRF channel (as the 'primary' channel) (Figure A5). For experiments involving transfection of eGFP-Fyn, eGFP Ack1, or eGFPTOM1L1, the intensity of each of TOM1L1, Ack1, or Fyn within CLSs is reported as the mean intensity within CLSs, normalized to the total intensity (of eGFP-Fyn, eGFP-Ack1 or eGFPTOM1L1) detected in the TIRF field (to normalize for variation in expression level), following selection of images that exhibited a restricted (low) level of eGFP-Fyn, eGFP-Ack1, or eGFPTOM1L1 expression level. Shown are the ensemble averages of various proteins within CLSs or frequency distribution of these values in individual structures, all were generated using GraphPad Prism.

\subsubsection{Identification of EGF-positive or eGFP-Fyn positive CLSs}

To identify a subpopulation of clathrin structures enriched in rhodamine-EGF or eGFPFyn we established a threshold of the 85th percentile of either EGF or Fyn fluorescence intensity within CLSs in the control (no inhibitor) condition in each experiment (Lucarelli et al., 2017; Delos Santos et al., 2017). Using this systematic threshold, we defined subsets of CLSs enriched in either fluorescent EGF or eGFP-Fyn each condition as those with ligand fluorescence intensity above this threshold. For the experiments in Figure 5.8, CLSs were first sorted by eGFP-Fyn 
status, then by rhodamine-EGF status. The fraction of eGFP-Fyn positive CLSs in each EGF status cohort is also reported.

Measurements (mean levels of various proteins within specified CLS subset for each cell) were subjected to either student's t-test or ANOVA followed by Tukey post-test with a threshold of $\mathrm{p}<0.05$ for statistically significant differences between conditions.

\subsection{Analysis of CCPs in Time-Lapse Image Series}

Automated detection, tracking and analysis of CCPs in time-lapse image series (as in (Figure 5.10 and Figure A4) was performed as previously described (Aguet et al., 2013; Mettlen and Danuser, 2014; Kadlecova et al., 2017) following TIRF microscopy of RPE cells stably expressing Tag-RFP-T-CLCa and transfected with the lowest detectable levels of eGFPFyn or eGFP-TOM1L1. All cells were treated with $20 \mathrm{ng} / \mathrm{mL}$ (unlabeled) EGF at the time of imaging. Diffraction limited clathrin structures were detected using a Gaussian-based model method to approximate the point-spread function (Aguet et al., 2013), and trajectories were determined from clathrin structure detections using the u-track software (Jaqaman et al., 2008). sCLSs were distinguished from bona fide CCPs as previously described, based on the quantitative and unbiased analysis of clathrin intensity progression in the early stages of structure formation (Aguet et al., 2013; Kadlecova et al., 2017). Both sCLSs and CCPs represent nucleation events, but only bona fide CCPs represent structures that undergo stabilization, maturation and in some cases scission to produce intracellular vesicles (Aguet et al., 2013; Kadlecova et al., 2017). Here, we report only CCP data. CCPs were sorted into eGFP-Fyn or eGFP-TOM1L1 positive cohorts, using methods for sorting CCP subpopulation by composition of a secondary channel established previously (Aguet et al., 2013), which effectively short CCPs 
based on Fyn or TOM1L1 content. We report the distribution of CCP lifetimes, as well as the 'plateau intensity' of eGFP-Fyn eGFP-TOM1L1 or RFP-CLC. CCPs exhibit several phases that can be described as initiation, growth/assembly, plateau and disassembly/scission (Loerke et al., 2011). Here we define the 'plateau intensity' of each marker as the mean fluorescence of that protein within each clathrin structure, measured within timepoints corresponding to $30 \%$ and $70 \%$ of the total lifetime of that structure, during which time CCPs exhibit minimal growth or disassembly. Because CCPs are diffraction-limited objects, the amplitude of the Gaussian model of the fluorescence intensity of RFP-CLC informs about CCP size. All measurements were subjected to ANOVA followed by Tukey post-test with a threshold of $\mathrm{p}<0.05$ for statistically significant differences between conditions. 


\section{Chapter 3. Determining the capacity in which clathrin is required for receptor signaling at the plasma membrane}

Concomitantly to receptor activation and signaling, EGFR is incorporated into small invaginating regions of the plasma membrane enriched in the scaffolding protein clathrin and the adaptor protein AP2. EGFR is responsible for the activation of several key intracellular signaling pathways following ligand engagement, including the Gab1/PI3K/Akt pathway, the PLC $\gamma$ pathway, and the mitogen-activated protein kinase (MAPK) pathway (Figure 1.2). While maximal activation of the Gab1/PI3K/Akt pathway occurs within 5 min of EGF stimulation, as majority of EGFR is resident within CCPs (Borisov et al., 2009), EGFR is able to sustain phosphorylation of the MAPK pathway downstream of it for hours following initial ligand interaction due to the ability of the receptor to also signal from intracellular compartments such as endosomes. (Pennock \& Wang, 2003; Platta \& Stenmark, 2011). Early studies of EGFR signaling using a dominant negative mutant of dynamin 2 revealed changes in EGF-stimulated signaling in cells in which dynamin was perturbed, which was one of the first clues that EGFR may exhibit distinct signaling properties at the plasma membrane compared to endosomes (Vieira et al., 1996). In addition, mutants of EGFR that exhibit negligible ubiquitination or that are deficient in AP2 binding do not become enriched within CCPs, and display defects in signaling including Akt phosphorylation (Goh et al., 2010). Collectively, these studies indicate that EGFR signaling is dependent on various proteins known to function in CME. This suggests that the process of receptor internalization may regulate EGFR signaling, or that these endocytosis proteins regulate EGFR signaling in a different manner.

In addition to evidence derived from perturbation of CME related proteins, there is also evidence that EGFR may exhibit distinct phosphorylation and protein binding when at the 
plasma membrane versus while resident on endosomes (Burke, Schooler, \& Wiley, 2001). Following internalization from the plasma membrane, phosphorylated EGFR accumulates on the endosomal membrane it is delivered to with its carboxyl terminus oriented to the cytoplasm so it can continue to signal to downstream effectors (Rush et al., 2012). Various signaling effectors involved in EGFR signaling are co-internalized with EGFR and remain associated with the receptor on the endosomal membrane, including Grb2, SHC, and Cb1 (Di Guglielmo et al., 1994; Levkowitz et al., 1998; Wang et al., 2002b). Additionally, EGFR residence at the endosome is required for EGFR-dependent apoptosis (Rush et al., 2012) and sustained MAPK signaling (Taub et al., 2007; Teis et al., 2006). Despite evidence that EGFR phosphorylation and protein interactions occur in endosomes and are distinct from that at the plasma membrane, the activation of Gab1/PI3K/Akt signaling is often rapid and transient, correlating largely with EGFR localization at the plasma membrane and within CCPs. Hence, how internalization of EGFR may regulate Gab1/PI3K/Akt signaling in particular is not well understood.

From this emerges the important question: how may clathrin regulate EGFR signaling at the plasma membrane? In addition, which aspects of the EGFR signal transduction mechanism, from receptor auto-phosphorylation to phosphorylation of Akt, may require clathrin? Providing an answer to these questions is the focus of this chapter.

For this chapter, my working hypothesis is that clathrin is required for receptor signaling from the plasma membrane, and specifically that its perturbation will disrupt EGF-stimulated Akt activation.

From this chapter, Figure 3.1, Figure 3.2, and Figure 3.3 are published in Garay et al., (2015). From this chapter, Figure 3.4 is published in Lucarelli et al., (2016). 


\section{1 siRNA Gene Silencing of CHC but not of Dynamin 2 Inhibits EGF-stimulated Akt Phosphorylation}

EGF stimulation of retinal pigment epithelial (ARPE-19, henceforth RPE) cells leads to the phosphorylation and subsequent activation of Akt, detectable following 5 minutes of stimulation with $20 \mathrm{ng} / \mathrm{mL}$ EGF and sustained up to 15 minutes following initial stimulation. To determine whether clathrin is required for EGF-stimulated Akt phosphorylation in RPE cells, I silenced $\mathrm{CHC}$ using siRNA. Cells subject to siRNA silencing of $\mathrm{CHC}$ were serum deprived for 1 hour prior to stimulation with $5 \mathrm{ng} / \mathrm{mL}$ EGF for 5, 10, or 15 minutes (as indictated), and immunoblotting whole cell lysates. Silencing of $\mathrm{CHC}$ led to a robust reduction in Akt phosphorylation at S473 following EGF stimulation compared to cells treated with control siRNA (Figure 3.1). These results are consistent with earlier studies that show either clathrin itself, or EGFR internalization by clathrin-mediated endocytosis, is required for EGF-stimulated Akt phosphorylation.

To resolve whether clathrin itself or CME is required for EGF-stimulated Akt phosphorylation, the GTPase responsible for scission of CCPs from the plasma membrane to yield intracellular CCVs, Dynamin 2, was also silenced using siRNA with an $89.9 \pm 3.0 \%(\mathrm{n}=4)$ reduction in total protein levels. Silencing of Dynamin 2 allows the formation of CCPs at the plasma membrane, however they remain resident here and do not internalize. In contrast to CHC silencing, Dynamin 2 silencing had no significant effect on EGF-stimulated Akt phosphorylation at S473 (Figure 3.1). Taken together, this result suggests a novel role for clathrin not only as an endocytic scaffolding protein critical for CME, but also a separate function, perhaps as a signaling regulator required for EGF-stimulated Akt phosphorylation that can be separated from the role of clathrin in endocytosis. Additionally, this reveals that internalization of EGFR into 
intracellular vesicles by CME is not required for EGF-stimulated Akt phosphorylation in RPE cells.

\subsection{Perturbation of Clathrin by Pitstop 2 or siRNA Gene Silencing does not alter EGFR Cell Surface Levels or Activation}

The reduction in Akt phosphorylation following silencing of $\mathrm{CHC}$ that I observed in Figure 3.1 could be due to a requirement for clathrin to support PI3K-Akt signaling subsequent to EGFR ligand binding and phosphorylation, or could instead be due to perturbation of EGFR itself, such as loss of cell surface EGFR or receptor ligand binding. In order to determine whether perturbation of clathrin affects EGFR activation upon EGF stimulation, I subjected RPE cells to siRNA silencing of CHC. RPE cells were serum starved for $1 \mathrm{~h}$ prior to stimulation with $5 \mathrm{ng} / \mathrm{mL}$ EGF for 5 or 10 mins (as indicated). Cells were then fixed and processed for immunofluorescent staining using an antibody that specifically recognizes EGFR only when phosphorylated on Y1068. Silencing of CHC exhibited no significant change in EGF-stimulated gain of EGFR phosphorylation at Y1068 compared to cells treated with control siRNA and stimulated with $5 \mathrm{ng} / \mathrm{mL}$ EGF for 5 minutes (Figure 3.2A). To complement siRNA, RPE cells were also treated with the pharmacological clathrin inhibitor, Pitstop 2, which prevents endocytic accessory proteins from binding to the $\mathrm{N}$-terminal domain of $\mathrm{CHC}$, therefore inhibiting the formation of new clathrin lattices upon addition of the drug, and thus inhibiting CME (Von Kleist et al., 2011). RPE cells were incubated with $10 \mu \mathrm{M}$ Pitstop 2 for 30 minutes following $1 \mathrm{~h}$ of serum deprivation and prior to stimulation with $20 \mathrm{ng} / \mathrm{mL}$ EGF. Next, whole cell lysates were prepared for immunoblotting. Compared to control cells treated with vehicle (DMSO), EGFstimulated phosphorylation of EGFR at key residues Y1068, Y1086, Y845, Y1173, and Y1045 
was unaffected by Pitstop 2 treatment (Figure 3.2B). Taken together, these results indicate that inhibition of Akt phosphorylation by perturbation of clathrin occurs at a later stage in the signaling pathway downstream of activation and autophosphorylation of EGFR.

One of the major ways by which the cell surface levels of EGFR are regulated is through $\mathrm{CME}$, for the purpose of signal attenuation or changes in the cell surface proteome in response to the varying needs of the cell. In order to determine whether cell surface protein levels of EGFR are affected by perturbation of CME, CHC and Dynamin 2 were again silenced in RPE cells using siRNA and total cell surface EGFR levels were probed immunofluorescently using an antiEGFR antibody in intact (non-permeabilized) cells. Notably, the anti-EGFR antibody used in this experiment recognizes an exofacial epitope on EGFR. Silencing of either CHC or Dynamin 2 did not significantly impact total cell surface EGFR levels compared for control siRNA treated cells (Figure 3.3). This confirms that the effect of clathrin perturbation on EGF-stimulated Akt phosphorylation is in fact due to the requirement for clathrin in signaling, and that clathrin perturbation did not lead to a reduction of Akt signal due to a reduction in EGFR cell surface levels.

\subsection{Clathrin is Required for HGF-Stimulated Akt Phosphorylation}

The requirement for clathrin in EGF-stimulated Akt phosphorylation does not reflect a requirement for EGFR internalization from the plasma membrane, as blocking EGFR endocytosis by perturbation of CHC or Dynamin 2 does not impact EGFR activation. Clathrin is also responsible for the endocytosis of a wide variety of other cell surface receptors, therefore I next aimed to determine whether this was an effect that is novel to EGFR by studying Met 
receptor signaling following stimulation with its ligand, Hepatocyte Growth Factor (HGF).

Met receptor is expressed in epithelial and endothelial tissues through development and into maturity, including in the breast epithelium, and is required for development of the placenta, liver, kidneys and skeletal muscle (Beviglia et al., 1997; Huh et al., 2004; Ponzo \& Park, 2010; Schmidt et al., 1995). Ligand binding by Met activates signaling downstream that induces epithelial cell dispersal, epithelial remodeling and invasive growth, all of which are all important during development and often involved in cellular transformation (Birchmeier et al., 2003). The Met receptor activates signaling pathways similar to EGFR downstream of its activation, also requiring recruitment and subsequent phosphorylation of the adaptor protein Gab1 (Organ \& Tsao, 2011; Rajadurai et al., 2012). Additionally and unlike EGFR, Met receptor is able to directly bind Gab1 following stimulation with HGF and phosphorylation of Y1349 on its Cterminus (Lock et al., 2000; Lock et al., 2003). Aberrant activation of Met leads to increased cancer cell invasion, a hallmark of aggressive tumors with poor prognosis (Cruz et al., 2003; Lengyel et al., 2005; Sawada et al., 2007). Additionally, amplification of Met has been identified as a mechanism of resistance to NSCLC treatment with EGFR kinase inhibitors erlotnib and gefitinib, implicating Met as an additional potential target in this disease (Bean et al., 2007; Engelman et al., 2007).

In order to determine whether Met receptor similarly requires clathrin for Akt phosphorylation, RPE cells were stimulated with $5 \mathrm{ng} / \mathrm{mL}$ of EGF or $50 \mathrm{ng} / \mathrm{mL}$ of HGF for 5 minutes following $1 \mathrm{~h}$ of serum deprivation, and whole cell lysates were prepared for immunoblotting. Compared to basal cells, HGF stimulation resulted in a similar increase in Gab1 (Y627) and Akt (S473) phosphorylation as EGF stimulation (Figure 3.4A). HGF stimulation did not elicit an increase in EGFR phosphorylation (Y1068) as expected, confirming that HGF is 
effective and specific to Met receptor in RPE cells (Figure 3.4A). To determine whether HGFstimulated Akt phosphorylation requires clathrin as EGF-stimulated Akt phosphorylation does, RPE cells were treated with Pitstop 2 and probed for phospho-Akt (S473) by immunoblotting whole cell lysates. Similarly to EGF-stimulated Akt phosphorylation, HGF-stimulated Akt phosphorylation was inhibited by treatment with Pitstop 2 (Figure 3.4B). These results suggest that the role of clathrin in supporting Gab1/PI3K/Akt signaling is not limited to EGFR, but also occurs for signaling by other RTKs, including HGF-stimulated activation of Met and subsequent Akt phosphorylation.

\subsection{Chapter 3 Summary}

This chapter outlines the role of clathrin in signaling and not just endocytosis. Using the pharmacological inhibitor Pitstop 2 complemented with siRNA gene silencing of $\mathrm{CHC}$ and Dynamin 2, I found clathrin to be necessary for PI3K/Akt signaling downstream of two distinct receptors in RPE cells, EGFR and Met, as quantified by levels of phospho-Akt (S473) following stimulation with EGF and HGF respectively. Furthermore, I found that perturbation of clathrin by siRNA gene silencing or treatment with Pitstop 2 did not alter cell surface levels of EGFR, and did not alter activation of the receptor following stimulation with EGF, as demonstrated both by western blot analysis and immunofluorescent staining of phospho-EGFR (Y1068). These experiments were done as part of a broader collaboration with other members of the Antonescu lab, resulting in the publication Garay et al., 2015. Here, we also find that EGF internalization is significantly impaired following perturbation of clathrin with siRNA or treatment with Pitstop 2, phosphorylated Gab1 becomes enriched within CCPs following EGF stimulation, and 
that expression of ErbB2 renders EGF-stimulated Akt phosphorylation insensitive to clathrin perturbation (Garay et al., 2015).

Together, these data demonstrate that clathrin may be regulating receptor signaling at the plasma membrane prior to CCP scission and vesicle formation, outlining a role for clathrin as a signaling scaffold and not just an endocytic scaffold protein. Clathrin may be responsible for coordinating the recruitment of specific cytosolic effectors and signaling regulators at the plasma membrane that are required for receptor signaling. 


\subsection{Chapter 3 Figures}
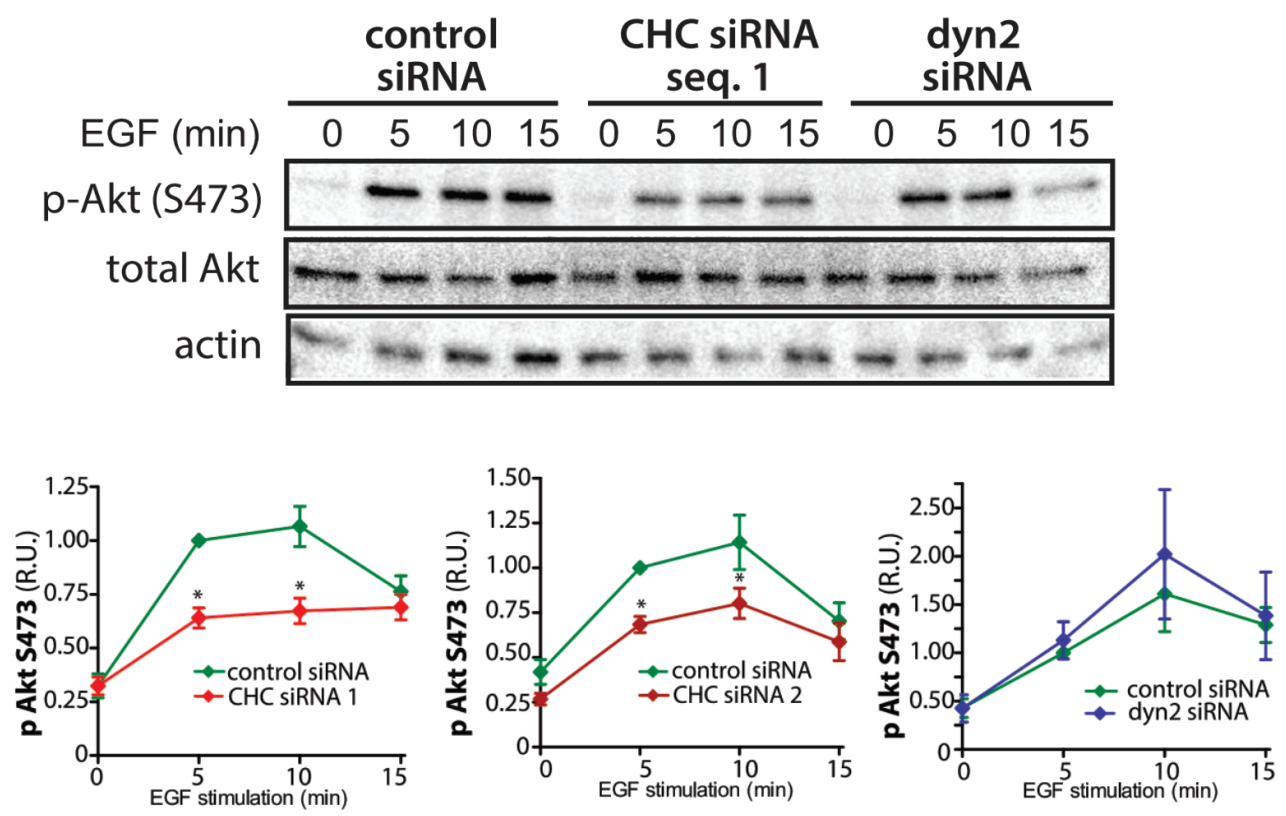

Figure 3.1 Silencing of clathrin but not Dynamin 2 reduces EGF-stimulated Akt phosphorylation.

ARPE-19 cells were transfected with siRNA targeting Dynamin 2 (dyn2), nontargeting (control), or one of two siRNA sequences targeting clathrin heavy chain (CHC siRNA 1, CHC siRNA 2). Following 1 hour of serum deprivation, cells were stimulated with $5 \mathrm{ng} / \mathrm{mL}$ EGF for 5, 10, or 15 mins, or left unstimulated (basal), and whole cell lysates were prepared. Lysates were immunoblotted and probed with anti-phospho-Akt (S473), anti-pan-Akt, or anti-pan-actin antibodies. Immunoblots were quantified as shown by mean $\pm \mathrm{SE}$ of $\mathrm{pS} 473$-Akt values, $\mathrm{p}<0.05$ relative to control EGF-stimulated condition. 


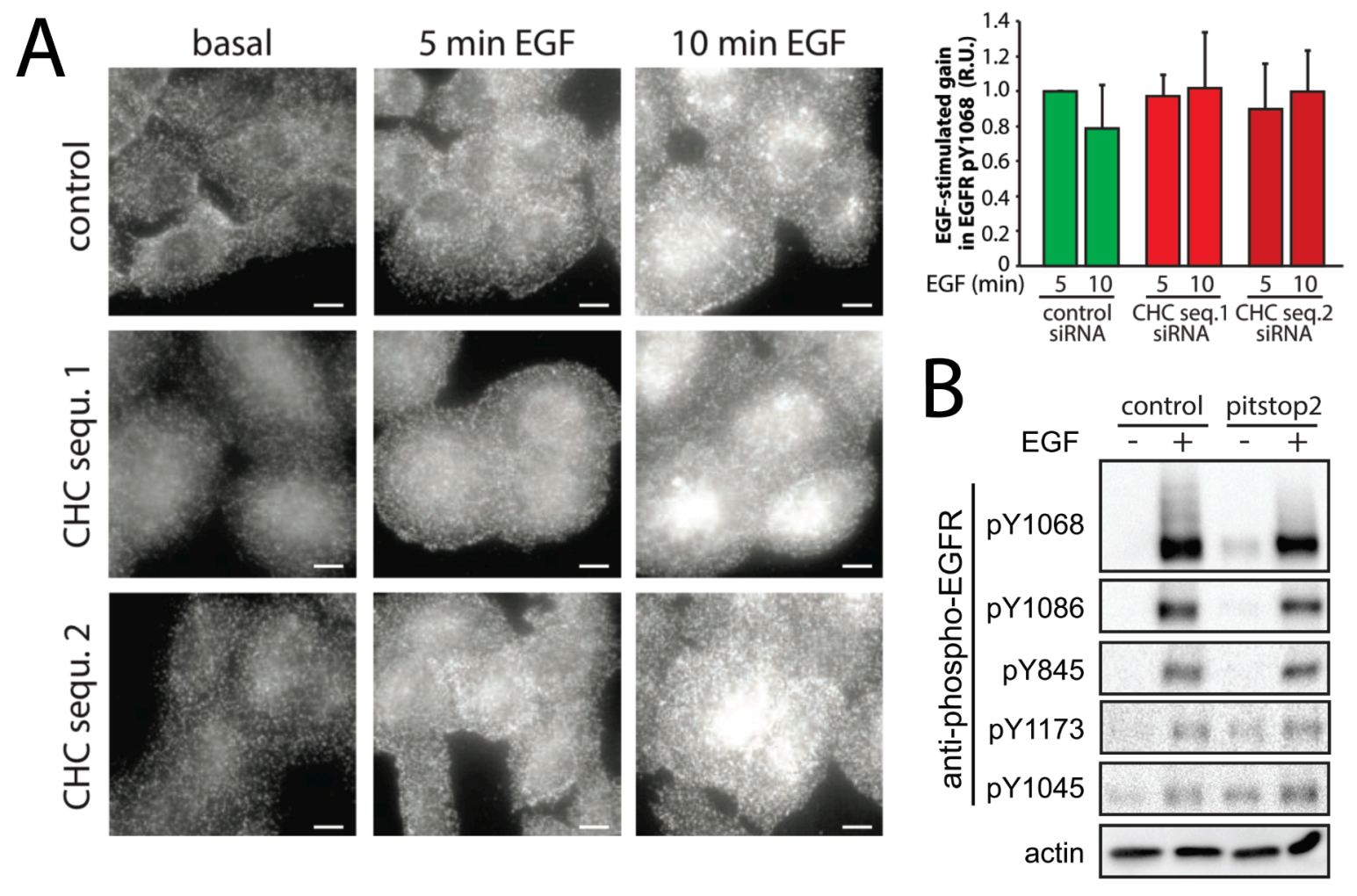

Figure 3.2 Perturbation of clathrin by pitstop2 treatment or siRNA gene silencing does not alter EGF-stimulated EGFR phosphorylation.

(A) RPE cells were transfected with one of two siRNA sequences targeting CHC (CHC siRNA 1, CHC siRNA 2) or nontargeting siRNA (control). Following $1 \mathrm{hr}$ serum deprivation, cells were stimulated with $5 \mathrm{ng} / \mathrm{mL}$ EGF for 5 or 10 minutes, fixed, and processed for immunofluorescent staining using an anti-phospho-EGFR (Y1068) antibody. Mean \pm SE of the EGF-stimulated gain in phospho-EGFR shown above ( $\mathrm{pY} 1068 ; \mathrm{n}=3$, minimum 20 cells per condition per experiment). Scale $=10 \mu \mathrm{M}$. (B) RPE cells were treated with vehicle control $(0.1 \%[\mathrm{vol} / \mathrm{vol}]$ DMSO) or $10 \mu \mathrm{M}$ Pitstop 2 for 30 minutes following $1 \mathrm{hr}$ serum deprivation and prior to stimulation with $5 \mathrm{ng} / \mathrm{mL}$ EGF for 5 minutes in the continued presence of the inhibitor. Whole cell lysates were prepared, resolved by immunoblotting, and probed with anti-phospho-EGFR antibodies recognizing pY1068, pY1086, pY845, pY1173, or pY1045 motifs, representative of three independent experiments. 


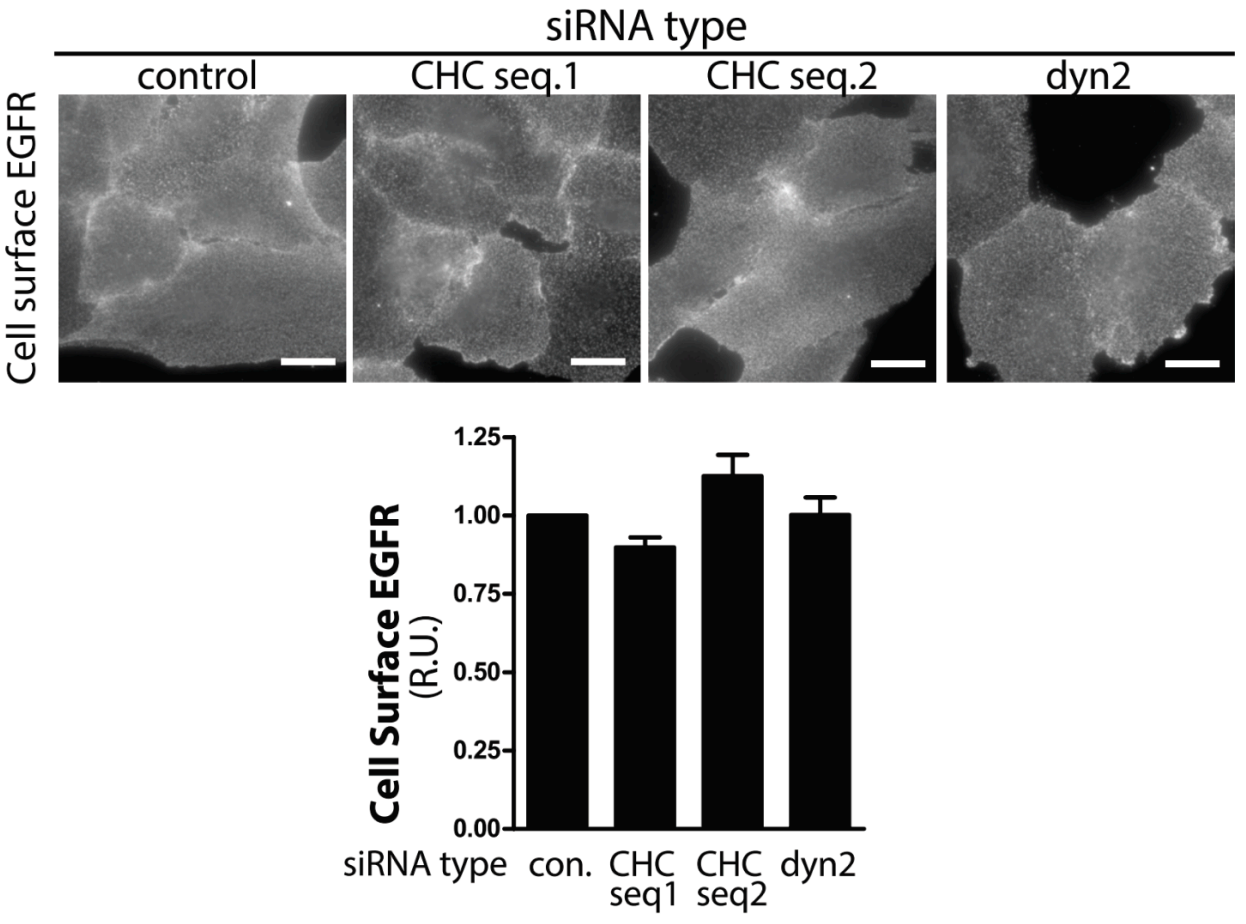

Figure 3.3 Silencing of clathrin or dynamin 2 does not affect cell surface EGFR.

RPE cells were transfected with Dynamin 2 siRNA (dyn2), one of two siRNA sequences targeting CHC (CHC siRNA 1, CHC siRNA 2) or nontargeting siRNA (control). Cells were left unstimulated and intact, fixed, processed for cell surface immunofluorescent staining using an anti-total-EGFR antibody. Relative fluorescence intensity of cell-surface EGFR (arbitrary units) was normalized to control siRNA treated cells and plotted for each treatment $(n=4)$. Representative fluorescence microscopy micrographs of cell surface EGFR staining. Scale, 20 $\mu \mathrm{m}$. 

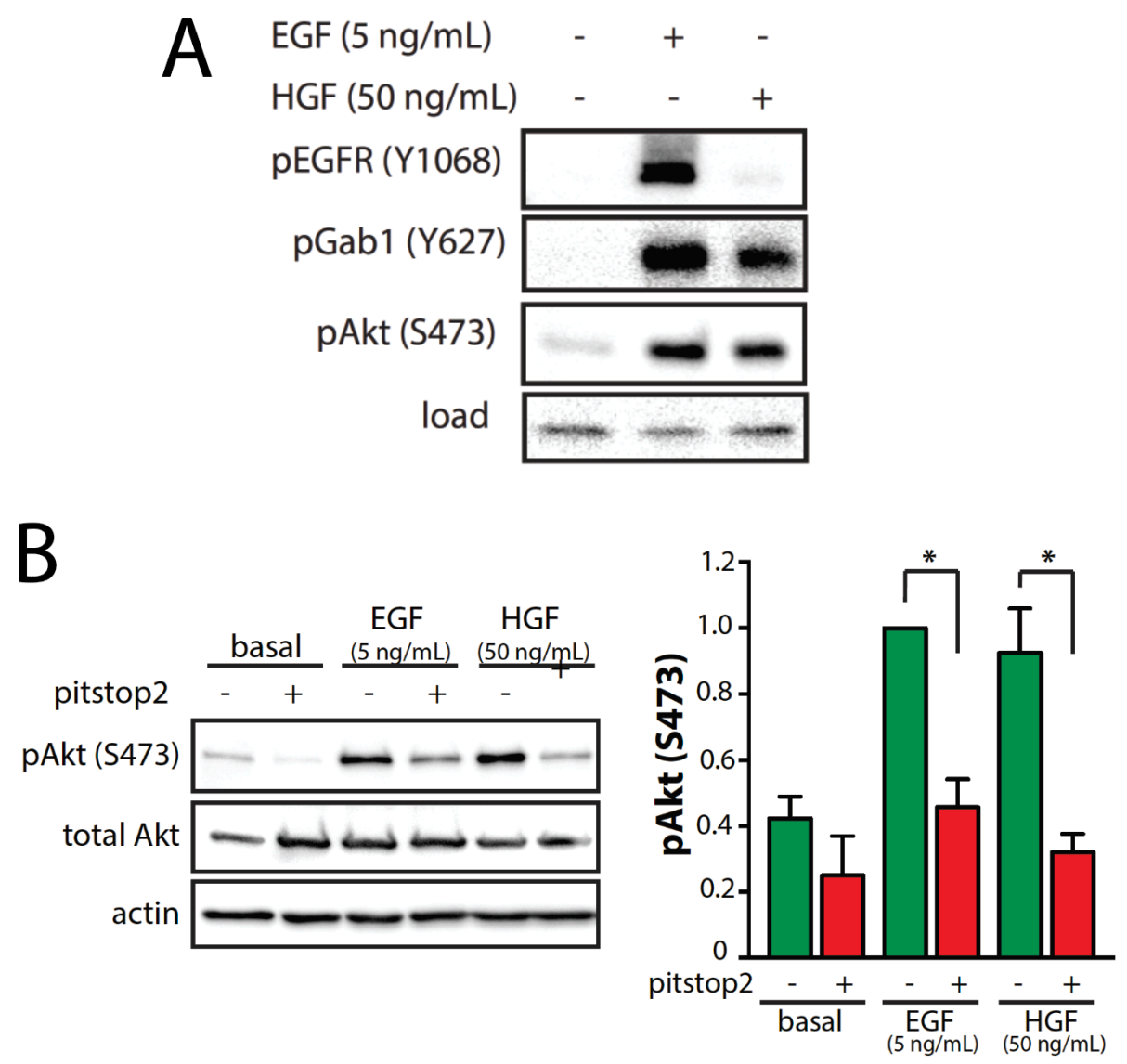

Figure 3.4 Clathrin is required for HGF-stimulated Gab1 and Akt phosphorylation.

(A) RPE cells were stimulated with $5 \mathrm{ng} / \mathrm{mL}$ EGF or $50 \mathrm{ng} / \mathrm{mL}$ HGF for 5 mins or left unstimulated (basal) following $1 \mathrm{hr}$ of serum deprivation. Whole cell lysates were prepared for immunoblotting and probed with anti-phospho-EGFR (Y1068), anti-phospho-Gab1 (Y627), antiphospho-Akt (S473), and pan-actin antibodies. (B) RPE cells were treated with $10 \mu \mathrm{M}$ Pitstop 2 or vehicle control $(0.1 \%$ DMSO) for 30 mins following $1 \mathrm{hr}$ of serum deprivation, prior to stimulation with $5 \mathrm{ng} / \mathrm{mL}$ EGF or $50 \mathrm{ng} / \mathrm{mL}$ HGF for 5 mins or left unstimulated (basal) in the continued presence of inhibitor. Whole cell lysates were prepared for immunoblotting and probed with anti-phospho-Akt (S473), anti-total-Akt and pan-actin antibodies. Quantified is the mean $\pm \mathrm{SE}$ of the pAkt values in each condition $(\mathrm{n}=4, *, \mathrm{p}<0.05)$ 


\section{Chapter 4. The impact of non-receptor tyrosine kinase perturbation on EGFR signaling}

EGFR transmits signals through the PI3K/Akt pathway, eventually leading to changes in cell metabolism, migration, proliferation, and survival (Lock et al., 2000; Kassenbrock et al., 2002). The most receptor proximal phosphorylation event that occurs downstream of EGFR activation is the recruitment of Gab1 to the receptor via interactions with Grb2, the latter of which directly binds phosphorylated EGFR (Lock et al., 2000). Gab1 becomes phosphorylated at multiple tyrosine residues downstream of EGF stimulation, however the mechanism by which this occurs and the kinases responsible for this remain unclear. Phosphorylation of Gab1 is a critical step in the PI3K/Akt pathway, as phosphorylation at Y447, Y472, and Y589 creates binding sites for the $\mathrm{p} 85$ regulatory subunit of PI3K, relieving its inhibitory action on the $\mathrm{p} 110$ catalytic subunit of the kinase and leading to a local increase in $\mathrm{PIP}_{3}$ production (HolgadoMadruga et al., 1996b; Mattoon et al., 2004a). Increased PIP $_{3}$ at the plasma membrane results in the translocation of Akt to the plasma membrane and its subsequent activation (Liu et al., 2018; Sugiyama et al., 2019). Given that clathrin is required for the EGF-stimulated phosphorylation of Gab1 and thus subsequent Akt phosphorylation, resolving the mechanism(s) that contribute to Gab1 phosphorylation, including identifying the kinase(s) responsible, is an important yet poorly understood phenomenon, which is the focus of Chapter 4 of this thesis.

SFKs have previously been implicated in the PI3K/Akt pathway; in vitro, the SFK c-Src phosphorylates EGFR on multiple tyrosine residues unique to the kinase, as well as aiding in the phosphorylation of autophosphorylation sites following EGF stimulation (Biscardi et al., 1999; Sato et al., 2013; Tice et al., 2002). However, previous studies of SFK signaling have relied on the relatively non-specific first generation TKIs PP1 and PP2, which have been shown to inhibit 
EGF-stimulated phosphorylation and activation of Akt at high concentrations (Kassenbrock et al., 2002). Treatment of cells with high concentrations of SFK inhibitors PP1 and PP2 block EGF-stimulated Akt phosphorylation, ubiquitination of EGFR, and phosphorylation of key proteins downstream of the receptor like Cbl (Kassenbrock et al., 2002). Both PP1 and PP2 have also been shown to have off-target effects that inhibit EGFR phosphorylation at concentrations as low as $1 \mu \mathrm{M}$ (Sorkina et al., 2002). Treatment of cells with PP1 and PP2 also inhibits EGF internalization by $30-40 \%$ in COS-1 and NIH $3 \mathrm{~T} 3$ cells, and in fibroblasts lacking Src, Yes, and Fyn, suggesting that at micromolar concentrations these inhibitors impose effects on endocytosis by inhibiting kinases other than SFKs (Sorkina et al., 2002). Since previous studies on SFK signaling have relied on inhibition of SFKs using high concentrations of PP1 or PP2 which have demonstrated off target effects, whether any SFKs are actually involved in clathrin-dependent signaling remains largely unknown.

Interestingly, the SFK Fyn is more distantly related to the other canonical endogenous SFKs c-Src and Yes, and is not able to directly bind EGFR. Instead, Fyn is dually lipidated and found within membrane rafts at the plasma membrane, and is also found associated with its activating and signaling adaptor, TOM1L1, which binds both Fyn and clathrin directly ( $\mathrm{Li}$ et al., 2005; Seykora et al., 2002; Collin et al., 2007). Given that TOM1L1 binds both Fyn and clathrin, Fyn is thus an interesting candidate to mediate clathrin-dependent control of PI3K-Akt signaling, but this has not been examined. In addition to Fyn, other cytosolic kinases could also potentially play a role in Gab1 phosphorylation and activation of PI3K/Akt signaling downstream of EGFR, such as Ack1. This kinase can directly bind clathrin (Shen et al., 2011), and has been found to be required for degradation of multiple RTKs, as well as modulation of processes such as cell migration, adhesion and spreading (Prieto-Echagüe \& Miller, 2011). 
In this chapter, I explore how SFKs are involved in EGF-stimulated activation of Akt, and whether a Src activating and signaling adaptor protein (TOM1L1), or other NRTKs that interact with clathrin (Ack1) play a role in the PI3K/Akt pathway.

\subsection{Fyn is required for EGF-stimulated Gab1 and Akt phosphorylation}

\subsubsection{Perturbation of SFKs by high dose Dasatinib inhibits Akt and Gab1 phosphorylation}

In order to determine whether and how SFKs may contribute to EGF-stimulated Akt phosphorylation, I chose to use the SFK inhibitor Dasatinib (N-(2-chloro-6-methyl- phenyl)-2(6-(4-(2-hydroxyethyl)- piperazin-1-yl)-2-methylpyrimidin-4- ylamino)thiazole-5-carboxamide); BMS-354825, Sprycel). Dasatinib is a second generation FDA-approved selective TKI that strongly targets $\mathrm{SFKs}\left(\mathrm{IC}_{50} 0.5 \mathrm{nmol} / \mathrm{L}\right)$, and also inhibits BCR-ABL fusion protein, stem cell factor receptor (c-KIT), and platelet-derived growth factor receptor (PDGFR) at higher concentrations. Dasatinib is currently used in the treatment of patients with Chronic Myelogenous Leukemia and Philadelphia chromosome-positive acute lymphoblastic leukemia (Gnoni et al., 2011).

Dasatinib has been found to be a more selective inhibitor of SFK signaling with less offtarget effects than PP1/PP2, therefore I aimed to determine if Dasatinib treatment also resulted in an inhibition of EGF-stimulated Akt phosphorylation as previous studies with PP1 and PP2 have. As an initial approach, RPE cells were incubated with $1 \mu \mathrm{M}$ Dasatinib for $30 \mathrm{mins}$ following $1 \mathrm{hr}$ of serum deprivation. Cells were then stimulated with $20 \mathrm{ng} / \mathrm{mL}$ EGF for $5 \mathrm{mins}$, whole cell lysates were prepared and subject to immunoblotting to detect the EGF-stimulated 
phosphorylation of SFKs, Gab1, and Akt. Compared to basal (non-EGF treated) cells, EGF stimulation caused a robust increase in phosphorylation at Y627 on Gab1 and S473 on Akt, and contributed to enhanced phosphorylation of Y307 on Gab1 as well (Figure 4.1A). In contrast, cells treated with $1 \mu \mathrm{M}$ Dasatinib exhibited a significant and dramatic inhibition of EGFstimulated phosphorylation of Akt and Gabl at both residues probed (Figure 4.1A). Importantly, $1 \mu \mathrm{M}$ Dasatinib did not significantly reduce phosphorylation of EGFR at Y1068, however we do see a loss of higher molecular weight species of EGFR following Dasatinib treatment compared to control and vehicle control treated conditions (Figure 4.1B).

Furthermore, while EGF stimulation did not yield an increase in SFK phosphorylation, treatment of cells with $1 \mu \mathrm{M}$ Dasatinib resulted in a loss of SFK phosphorylation in both basal and EGF-stimulated cells. Quantification of phosphorylation intensity shows approximately an $80 \%$ reduction $\left(\mathrm{p}<0.05^{* *}\right)$ of EGF-stimulated Akt phosphorylation in cells treated with $1 \mu \mathrm{M}$ Dasatinib as well as a $60 \%$ reduction $\left(\mathrm{p}<0.05^{*}\right)$ of SFK phosphorylation at activating residue Y416.

There are three binding sites present on Gab1 that mediate association with the $\mathrm{p} 85$ regulatory subunit of PI3K; Y447, Y472 and Y589 (Mattoon et al., 2004). There are, however, no phospho-specific antibodies available for probing these sites, and therefore surrogate phosphorylation sites on the protein such as Y627 and Y308 have been used to infer activity. These represent potential binding sites for Shp2 and PLC $\gamma$ respectively (Chan et al., 2010). By probing these sites, overall activation of Gab1 can be inferred for its ability to bind and activate PI3K. Compared to basal cells, EGF stimulation causes a robust increase in phosphorylation at Y627 on Gab1 and contributed to enhanced phosphorylation of Y307 on Gab1 as well. Quantification of phosphorylation intensity shows approximately a $70 \%$ reduction $\left(\mathrm{p}<0.05^{* *}\right)$ of 
EGF-stimulated Gab1 phosphorylation at Y627 in cells treated with $1 \mu \mathrm{M}$ Dasatinib (Figure 4.1A, C), and a similar reduction in phosphorylation of Y308. This data suggests that following EGF stimulation, Dasatinib is able to inhibit PI3K/Akt signaling upstream of Akt activation at the level of Gab1 phosphorylation. Whether the kinase responsible for phosphorylating Gab1 is directly being inhibited by Dasatinib, or whether the Dasatinib-inhibited kinase acts further upstream in the pathway remains to be determined.

\subsubsection{Perturbation of SFKs by low dose Dasatinib inhibits Src phosphorylation, but not} Akt or Fyn

Using a relatively high dose of Dasatinib $(1 \mu \mathrm{M})$, I was able to confirm that phosphorylation of SFKs on a conserved motif (Y416 in c-Src) and activation of Akt downstream of EGF stimulation were inhibited. However, although $1 \mu \mathrm{M}$ is the standard concentration of Dasatinib used in previous reports (Nautiyal et al., 2009), the IC 50 of c-Src for Dasatinib has been found to be $0.5 \mathrm{nM}$ (Lombardo et al., 2004), and inhibition of c-Src using Dasatinib has been previously achieved at concentrations as low as $50 \mathrm{nM}$ (Nam et al., 2005). Hence, I next aimed to investigate whether a dose curve of Dasatinib can allow me to determine if the sensitivity of SFKs and Akt phosphorylation to Dasatinib treatment is similar. If their sensitivities are similar, this may suggest that SFKs are involved in Akt activation.

To do this, RPE cells were incubated with various lower concentrations of Dasatinib (ranging from $50 \mathrm{nM}$ up to $250 \mathrm{nM}$ ) for 30 minutes following $1 \mathrm{~h}$ of serum deprivation. Following this and in the continued presence of inhibitor, cells were stimulated with $20 \mathrm{ng} / \mathrm{mL}$ 
EGF for 5 mins or left unstimulated (basal). Whole cell lysates were subjected to immunoblotting to detect the EGF-stimulated phosphorylation of SFKs and Akt.

If the SFK(s) detectable by the phospho-Src (Y416) antibody is required for Akt activation downstream of EGF stimulation, inhibition of the SFK(s) by Dasatinib would result in inhibition of Akt and SFK phosphorylation. Treatment of cells with a range of concentrations of Dasatinib revealed different effects of the drug on the EGF-stimulated phosphorylation of SFKs and Akt, despite previous research distinguishing certain members of the SFKs as the primary mediators of Akt signaling in response to EGF (Kassenbrock et al., 2002). For instance, following treatment of cells with the lowest dose of Dasatinib tested (50 nM), EGF-stimulated Akt phosphorylation was not significantly affected, whereas phosphorylation of SFKs was nearly completely abrogated (Figure 4.2). This is the first time a disconnect in the functional requirements for EGF-stimulated Akt activation and SFKs has been shown. Quantification of the phosphorylation intensity of both proteins further highlights their differences. At the critical 50 nM Dasatinib concentration, there is no significant reduction in Akt phosphorylation at S473, whereas the phosphorylation of SFKs (detected by the phospho-Src Y416 antibody) show a greater than $60 \%$ reduction $(\mathrm{p}<0.05 *)$ at the same concentration (Figure 4.2).

Immunoblotting with the phospho-Src (Y416) antibody often exhibited only one visible band on immunoblots that likely corresponds to phospho-c-Src, the canonical SFK, despite the fact that the antibody is reported by the manufacturer to also recognize the phosphorylated forms of other SFKs. The phosphorylation of other SFKs is detectable by examining lower molecular weights, such as $59 \mathrm{kDa}$ for Fyn, since c-Src is noticeably larger at approximately $65 \mathrm{kDa}$. That this canonical antibody for detection of phosphorylated forms of SFKs (from Cell Signaling) only detects a single band despite the fact that various SFKs have molecular weights that differ 
sufficiently to allow distinct bands to be resolved by western blotting suggests that this antibody only recognizes a single specific phosphorylated SFK in these whole-cell lysates. By referencing molecular weight markers on immunoblotted membranes, it was determined that the visible band being recognized appeared at $65 \mathrm{kDa}$ and therefore may only be representative of phospho-c-Src and not other SFKs, suggesting that the phosphorylated forms of other SFKs cannot simply be determined by immunoblotting with this antibody. Additionally, Fyn has been previously shown to be phosphorylated in response to EGF stimulation, however, the immunoblots in Figure 4.1 show no detectable EGF-stimulated gain in SFK phosphorylation. Hence, how lower concentrations (50 nM) of Dasatinib impact the activity of other SFKs in the cell, in particular that of Fyn, remains unknown. Together, these results show that c-Src may not be required for the activation of Akt upon EGFR activation, and suggests that another member of the SFKs could instead potentially be functioning in the PI3K/Akt pathway.

The lack of effect of Dasatinib treatment on EGF-stimulated Akt phosphorylation at lower Dasatinib doses which effectively block the activity of some SFKs could be due to either a role for a Dasatinib-insensitive SFK, or a requirement for a different cytosolic kinase. I next aimed to determine whether Dasatinib treatment inhibited the phosphorylation of SFK Fyn by using an antibody specific to the phosphorylated form of this kinase and not other SFKs (Githaka et al., 2016). If Fyn is as sensitive to Dasatinib as c-Src, I would expect to see an inhibition of Fyn phosphorylation following treatment with similar concentrations of Dasatinib to that in Figure 4.2. $\mathrm{RPE}$ cells were incubated with either the lowest $(50 \mathrm{nM})$ or the highest $(250 \mathrm{nM})$ concentration of Dasatinib used in the previous dose curve for 30 minutes following $1 \mathrm{hr}$ of serum deprivation, and stimulated with $20 \mathrm{ng} / \mathrm{mL}$ EGF for 5 minutes in the continued presence of inhibitor. Whole cell lysates were then prepared and subjected to immunoblotting with an 
antibody to detect phospho-Fyn (Y420), which corresponds to the Y416 motif on c-Src, or Actin (loading control). In contrast to the Cell Signaling anti-phospho-SFK antibody used previously (Figure 4.1 and Figure 4.2), the anti-phospho-Fyn (Y420) antibody was able to detect an EGFstimulated gain in phosphorylated Fyn, consistent with the literature (Figure 4.3A).

Additionally, following treatment with either $50 \mathrm{nM}$ or $250 \mathrm{nM}$ Dasatinib, there is no significant change in phosphorylation of Fyn compared to control cells. These results suggest that Fyn may be less sensitive to inhibition by Dasatinib than other SFKs detected by the phospho-Src (Y416) antibody, and that Fyn may be required for EGF-stimulated Akt activation.

\subsubsection{Fyn siRNA inhibits EGF-stimulated Gab1 and Akt phosphorylation}

In determining how SFKs may regulate EGF-stimulated Akt activation, I complemented pharmacological inhibition of SFKs using Dasatinib with siRNA gene silencing of specific SFKs, in particular, of Fyn. Since I was able to determine that Fyn but not other SFKs such as cSrc may be a Dasatinib-insensitive and activated in response to EGF stimulation, I next aimed to determine the specific role of Fyn in the activation of the PI3K/Akt pathway upon EGF stimulation. To do this, I used siRNA gene silencing of Fyn and measured the EGF-stimulated phosphorylation of PI3K/Akt pathway intermediates by western blotting.

The effectiveness of the siRNA sequence designed to target Fyn was first assessed. RPE cells were first treated with siRNA targeting Fyn (or control siRNA), and whole cell lysates were prepared for western blotting and immunostaining with antibodies recognizing both total Fyn protein and phospho-Fyn (Y420). Silencing of Fyn by siRNA resulted in an $87 \%$ decrease in total Fyn protein levels in the cell, and a significant reduction in EGF-stimulated levels of 
phosphorylated Fyn as detected by the anti-phospho-Fyn (Y420) antibody. This result indicates that the siRNA sequence designed was specific and capable of knocking down endogenous Fyn protein levels, and also supports that the anti-phospho-Fyn antibody indeed preferentially detects phosphorylated Fyn (Y420) (Figure 4.3B).

Following this, I subjected whole cell lysates to immunoblotting with antibodies targeting phospho-Gab1 (Y627), phospho-Akt (S473) and total Akt to determine how Fyn may control EGFR signaling. RPE cells subject to siRNA knockdown of Fyn displayed a substantially impaired ability to phosphorylate Gab1 (Y627) and Akt (S473) in response to EGF stimulation. These results highlighting a role for Fyn in EGF-stimulated activation of PI3K/Akt signaling (Figure 4.3C). These results also indicate that EGF stimulation may preferentially engage Fyn, and that Fyn may be less Dasatinib-sensitive than other SFKs.

\subsection{TOM1L1 is required for EGF-stimulated Akt phosphorylation}

\subsubsection{TOM1L1 siRNA inhibits EGF-stimulated Gab1 and Akt phosphorylation}

Since Fyn silencing has a similar phenotype to clathrin silencing or inhibition in the context of EGFR signaling, I next aimed to determine how the clathrin-binding and Fyn adaptor protein TOM1L1 contributes to EGFR signaling. Importantly, TOM1L1 is able to bind Fyn, EGFR, and also interacts directly with clathrin using two separate motifs within its C-terminal region (Li et al., 2005; Seykora et al., 2002; Collin et al., 2007). Since Fyn is not known to directly bind to clathrin or EGFR, TOM1L1 may be effectively bridging the gap between EGFR resident within CCPs and Fyn at the plasma membrane, allowing Fyn to trigger PI3K/Akt signaling by localized phosphorylation of Gab1. TOM1L1 becomes tyrosine phosphorylated 
following EGF stimulation; however this effect is ablated when Fyn is inhibited (Li et al., 2005; Liu et al., 2009), suggesting that in addition to binding and activating Fyn, TOM1L1 may also be subject to reciprocal phosphorylation by Fyn.

In order to determine the contribution of TOM1L1 to EGFR signaling, I sought to silence TOM1L1 using siRNA and assess its effect on the activation of the PI3K/Akt pathway upon EGF stimulation. First, the effectiveness of the siRNA designed to silence TOM1L1 was tested by treating RPE cells with TOM1L1 siRNA and processing whole cell lysates for immunoblotting using an antibody specific for TOM1L1. TOM1L1 silencing resulted in an $88 \%$ reduction in total endogenous TOM1L1 as detected by the antibody, supporting both the effectiveness of the siRNA and the specificity of the antibody for TOM1L1 (Figure 4.4A). Silencing of TOM1L1 significantly impaired EGF-stimulated Akt phosphorylation (S473) compared to cells treated with control siRNA, similar to that of Fyn and clathrin silencing (Garay et al., 2015) (Figure 4.4B). Perturbation of Fyn and TOM1L1 by siRNA display a similar phenotype in Akt phosphorylation to that of clathrin siRNA, suggesting that these proteins may be working in concert with plasma membrane clathrin structures to control EGFR signaling leading to Akt activation.

\subsubsection{Fyn and clathrin binding by TOM1L1 are required for EGF-stimulated Akt phosphorylation}

In order to further probe the mechanism by which TOM1L1 influences EGF-stimulated Akt phosphorylation, I next aimed to determine whether the ability of TOM1L1 to bind clathrin or Fyn is required for its the activation of PI3K/Akt signaling upon EGF stimulation. Mutants of 
TOM1L1 that are defective in their ability to bind clathrin (clathrin-binding mutant: ${ }^{447} \mathrm{FDPL}^{450}$ to ${ }^{447} \mathrm{AAAA}^{450}$ ) and bind Fyn (Y460F) were obtained (Liu et al., 2009), and transfected into RPE cells, with the aim that these overexpressed proteins would act in a dominant-interfering manner with regards to endogenous TOM1L1. The cells were stimulated with $20 \mathrm{ng} / \mathrm{mL}$ EGF following $1 \mathrm{hr}$ of serum deprivation, then fixed and subjected to immunofluorescent staining to detect phospho-Akt (S473) as well as transfected HA-tagged TOM1L1 (using an anti-HA antibody). Cells overexpressing wildtype TOM1L1 exhibited an increase in Akt phosphorylation following EGF stimulation, similar to that seen in untransfected cells (Figure 4.5). In contrast, cells overexpressing either clathrin-binding or Fyn-binding mutants of TOM1L1 failed to elicit an increase in Akt phosphorylation upon EGF stimulation, demonstrating that these interactions are necessary for propagation of PI3K/Akt signaling downstream of EGFR activation, and confirming that the mutants were in fact acting in a dominant interfering fashion (Figure 4.5). Taken together, I have determined that the ability of TOM1L1 to directly bind Fyn and clathrin is required for EGF-stimulated Akt phosphorylation, outlining a mechanism by which TOM1L1 may be eliciting these effects via interacting with endocytic adaptor and accessory proteins at the level of the clathrin coated pit.

\subsection{TOM1L1 or Fyn siRNA do not affect phospho-EGFR or total cell surface EGFR}

In Chapter 3, I examined how clathrin perturbation impacts EGF-stimulated Akt phosphorylation. In doing so, I resolved that the loss of clathrin function did not affect EGFstimulated EGFR phosphorylation, but instead impacted a stage subsequent to EGFR phosphorylation, of which the most upstream perturbation detected was Gab1 phosphorylation. 
Given that the ability of TOM1L1 to bind clathrin was required to sustain EGF-stimulated Akt phosphorylation (Figure 4.5), I next sought to examine if the reduction in Akt and Gab1 phosphorylation by silencing Fyn or TOM1L1 occurred due to loss of signal transduction following normal EGFR phosphorylation (as is the case for clathrin perturbation), or if this instead could be due to an alteration in EGFR activation or phosphorylation itself.

In order to determine whether perturbation of Fyn or TOM1L1 affect EGF-stimulated EGFR activation, Fyn or TOM1L1 were silenced in RPE cells using siRNA, followed by stimulation with $5 \mathrm{ng} / \mathrm{mL}$ EGF for 3 or 5 minutes (as indicated in Figure 4.6). Whole cell lysates were prepared and probed with an anti-phospho-EGFR (Y1068) antibody to assess phosphorylation at this key residue involved in EGF-stimulated activation of PI3K/Akt signaling. Compared to cells treated with a control siRNA sequence, EGF-stimulated phosphorylation of EGFR Y1068 was not significantly affected by either Fyn or TOM1L1 silencing (Figure 4.6AB). Additionally, in order to determine whether internalization or recycling of EGFR were altered by either Fyn or TOM1L1 perturbation, total cell surface EGFR levels were probed following immunofluorescence staining of intact (unpermeabilized) cells in the basal (non-EGF stimulated) condition using an anti-EGFR antibody that selectively recognizes the EGFR ectodomain. Silencing of either Fyn or TOM1L1 did not significantly impact total cell surface EGFR levels compared for control siRNA treated cells (Figure 4.6C-D). Taken together, these results indicate that the effect of Fyn or TOM1L1 perturbation on EGF-stimulated Akt and Gab1 phosphorylation is likely due to the requirement for these proteins in signaling downstream of normal EGFR activation and phosphorylation upon EGF binding, and not due to changes in the receptor itself. 


\subsection{Ack1 is required for EGF-stimulated Gab1 and Akt phosphorylation}

In determining candidates for a kinase able to potentially regulate Gab1 phosphorylation and Akt activation in the PI3K/Akt pathway downstream of EGFR, it is important to consider kinases that in some way are able to interact with clathrin, other endocytic proteins, or EGFR itself. Thus far, Fyn and TOM1L1 have met these requirements and perturbation of either protein results in a significant reduction in EGF-stimulated Akt phosphorylation, however this effect was not complete. I observed approximately a $60 \%$ reduction in relative phosphorylation of EGFstimulated Akt and Gab1 for each of TOM1L1 and Fyn silencing. It is important therefore to explore the possibility that Fyn and its adaptor protein TOM1L1 may not be the only NRTK functioning in activation of the PI3K/Akt pathway upon EGF stimulation, and that there may be more than one kinase responsible for regulating phosphorylation of Gab1 downstream of EGFR activation. Ack1 is a NRTK known to regulate EGFR signaling and endocytosis and is able to bind clathrin as well as other intermediates of the PI3K/Akt pathway directly (Prieto-Echagüe \& Miller, 2011; Grovdal et al., 2008). The exact nature of the involvement of Ack1 in EGFstimulated Akt phosphorylation however remains largely unexplored. I therefore next aimed to determine if Ack1 is involved in EGF-stimulated Akt phosphorylation by performing siRNA gene silencing of Ack1 in RPE cells similarly to what was done for Fyn and TOM1L1. Ack1 was

silenced, cells were stimulated with $5 \mathrm{ng} / \mathrm{mL}$ EGF for 3 or 5 mins (as indicated) following $1 \mathrm{hr}$ of serum deprivation, and whole cell lysates were prepared for immunoblotting using anti-phosphoAkt (S473) and anti-total Akt antibodies. Quantification of three separate experiments confirmed that there was a significant reduction (50\%) of Akt phosphorylation at S473 following EGF stimulation in Ack1 siRNA treated cells compared to cells treated with control siRNA (Figure 
4.7A), similar to that of Fyn, TOM1L1, or clathrin silencing. This confirms that there is a functional role for Ack1 in clathrin-dependent EGF-stimulated Akt phosphorylation.

Since both Fyn and Ack1 were found to be required for EGF-stimulated Akt phosphorylation, this raises the questions of whether these NRTKs are acting in parallel pathways to activate Akt, or potentially acting downstream of one another in the same pathway. In order to differentiate between these two possibilities, Ack1 and Fyn were both silenced in RPE cells and the effect on EGF-stimulated Akt activation downstream was probed. Ack1 and Fyn were silenced, cells were stimulated with $5 \mathrm{ng} / \mathrm{mL}$ EGF for 3 or 5 mins (as indicated), and whole cell lysates were prepared for immunoblotting using anti-phospho-Akt (S473) and antitotal Akt antibodies. Following siRNA, we see that the effect of perturbing Fyn and Ack1 together did not have an additive effect on EGF-stimulated Akt phosphorylation, as perturbation of either kinase alone exhibited a similar inhibition as that of both together (Figure 4.7B). This suggests that Fyn and Ack1 may be acting in the same pathway, rather than in separate parallel pathways to activate Akt.

\subsection{Fyn and TOM1L1 are required for EGF-stimulated Akt phosphorylation in MCF10A cells}

To determine whether these effects observed in RPE cells are apparent in other cell lines, I chose to examine EGF-stimulated Akt phosphorylation in three additional cell types: a nontransformed, mammary gland epithelial cell line called MCF10A, and two breast cancer cell lines, T47D and SUM149PT. I sought to silence Fyn and TOM1L1 using siRNA in each cell line and assess its effect on the activation of the PI3K/Akt pathway upon EGF stimulation. Silencing of either Fyn or TOM1L1 significantly impaired EGF-stimulated Akt phosphorylation (S473) in 
MCF10A cells compared to cells treated with control siRNA (Figure 4.8A), similar to that of clathrin, Fyn, or TOM1L1 silencing in RPE cells (Figure 4.3, Figure 4.4) (Garay et al., 2015). However, silencing of Fyn or TOM1L1 in the breast cancer cell lines T47D and SUM149PT did not significantly alter EGF-stimulated Akt phosphorylation (Figure 4.8B-C). This suggests that while some cell lines require Fyn and TOM1L1 for EGF-stimulated Akt phosphorylation, this is not a ubiquitous requirement across all cell types, suggesting that they utilize alternative mechanisms of activating Akt.

\subsection{Chapter 4 Summary}

This chapter outlines the role of two NRTKs, Fyn and Ack1, and that of the clathrinbinding Fyn adaptor protein TOM1L1 in EGF-stimulated Akt phosphorylation. Since canonical SFK inhibitors PP1 and PP2 have off target effects at higher concentrations, I explored the effect of low concentrations of Dasatinib on EGFR signaling and determined that there is no clear evidence for a role of SFKs in EGF-stimulated Akt phosphorylation. Activation of the SFK(s) recognized by the phospho-Src (Y416) antibody was abolished at concentrations as low as 50 $\mathrm{nM}$. In contrast however, Akt phosphorylation persisted in treatments up to $250 \mathrm{nM}$ of Dasatinib, revealing a functional disconnect between activation of Akt and the activity of certain SFKs that up until now has not been demonstrated. I then determined that the antibody being used to detect phosphorylated SFKs was likely preferentially representative of phospho-c-Src and not of other SFKs such as Fyn that have lower molecular weights than c-Src, and therefore an antibody specific for the phosphorylated SFK Fyn was used in subsequent experiments. Following treatment with $50 \mathrm{nM}$ or $250 \mathrm{nM}$ Dasatinib and stimulation with EGF, Fyn phosphorylation was not significantly affected by Dasatinib and was also found to be phosphorylated in response to 
EGF. Silencing of Fyn as well as of the clathrin-binding Fyn adaptor protein TOM1L1 using siRNA revealed a functional requirement for each in EGF-stimulated Gab1 and Akt phosphorylation. Additional cytosolic kinases may also play a role in regulating clathrindependent EGFR signaling, therefore siRNA silencing of Ack1 was also performed to assess how this perturbation impacted Akt phosphorylation. Ack1 silencing by siRNA resulted in a significant reduction in Akt phosphorylation downstream of EGF stimulation, phenocopying the requirement of clathrin, Fyn, and TOM1L1 in this pathway. Taken together, these results indicate that non-receptor tyrosine kinases Fyn and Ack1, and the adaptor protein TOM1L1 all play novel roles in regulating PI3K/Akt signaling downstream of EGFR, and that there may be more than one kinase acting in the pathway in a clathrin-dependent manner. 


\subsection{Chapter 4 Figures}

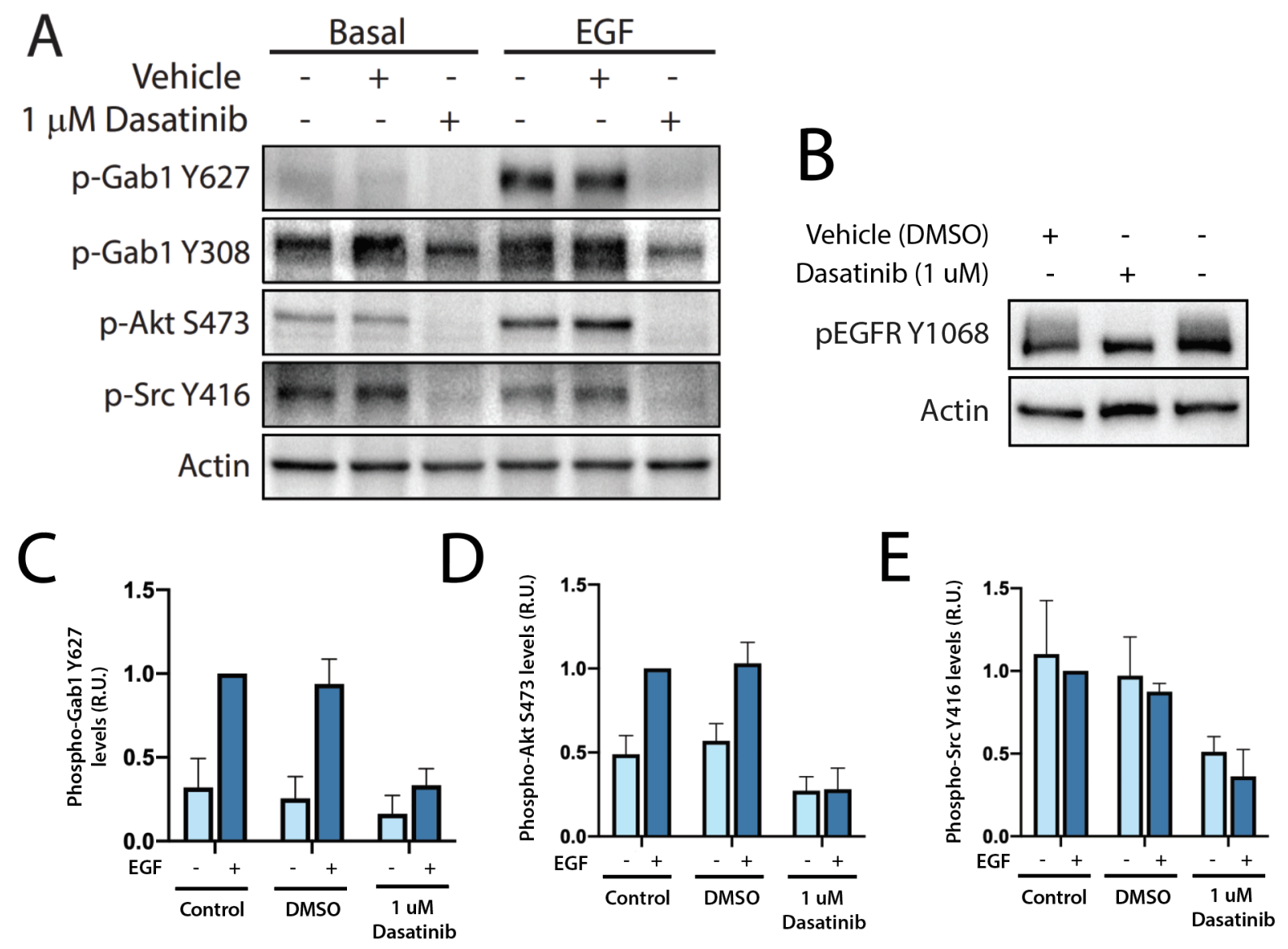

Figure 4.1 High-dose Dasatinib inhibits SFKs and activation of the PI3K/Akt pathway.

RPE cells were treated with Dasatinib $(1 \mu \mathrm{M})$ or vehicle control $(0.1 \%$ [vol/vol] DMSO $)$ for 30

minutes following 1 hour of serum deprivation. Cells were then either stimulated with $20 \mathrm{ng} / \mathrm{mL}$ EGF for 5 mins or left unstimulated (basal). Whole cell lysates were prepared for immunoblotting and probed with (A) anti-phospho-Gab1 (Y308 and Y627), anti-phospho-Akt (S473), anti-phospho-Src (Y416), (B) anti-phospho-EGFR (Y1068) and anti-actin antibodies. Shown are representative immunoblots and the mean $\pm \mathrm{SE}$ signal intensity (from $\mathrm{n}>3$ independent experiments; ${ }^{*}, \mathrm{p}<0.05$ relative to control (no vehicle) EGF-stimulated condition). (C-E) Immunoblots were quantified as shown by mean \pm SE of respective antibody values, $\mathrm{p}<$ 0.05 relative to control (no vehicle) EGF-stimulated condition. 

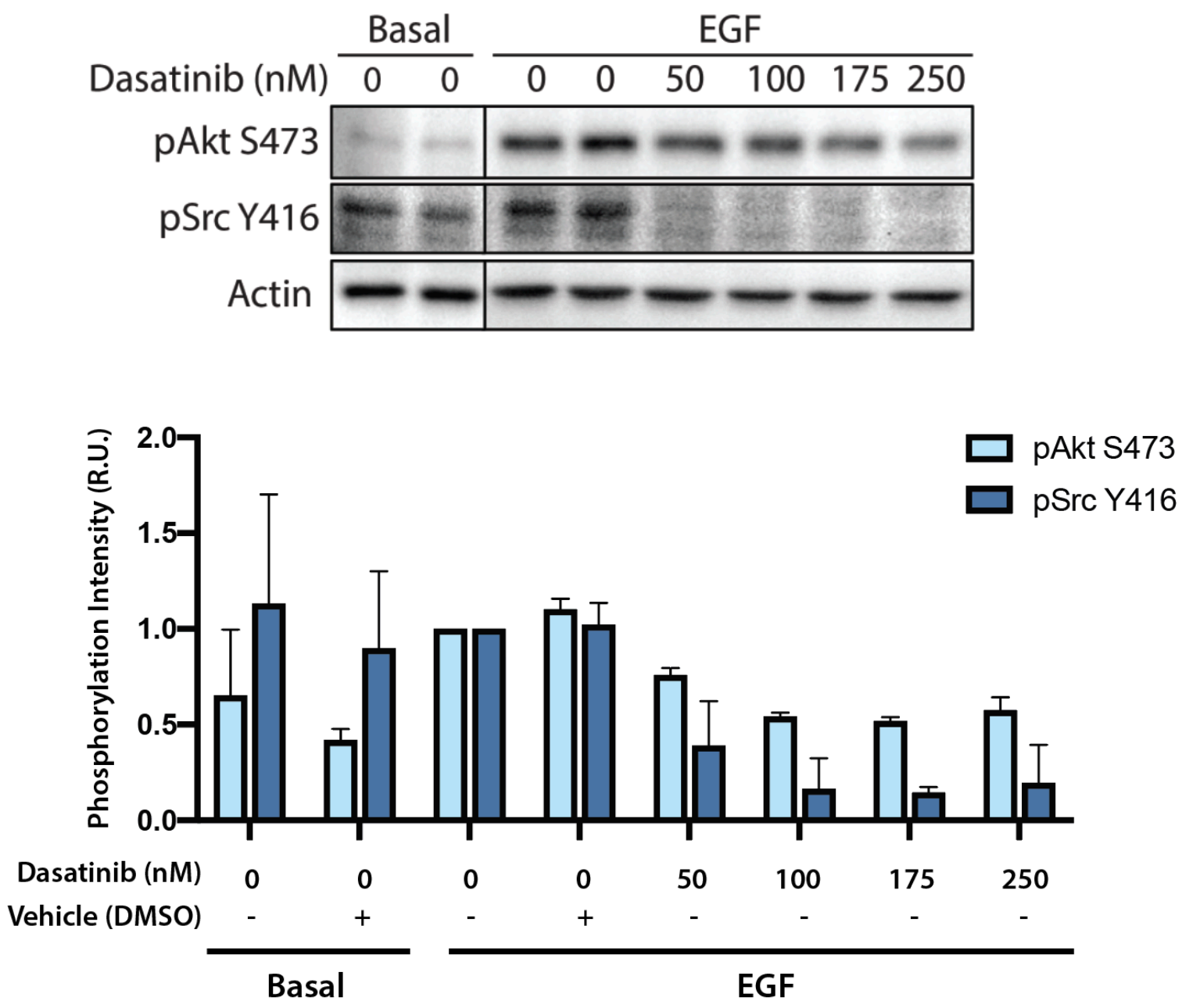

Figure 4.2 Akt phosphorylation is less sensitive to low-dose Dasatinib than c-Src.

RPE cells were treated with Dasatinib $(50 \mathrm{nM}-250 \mathrm{nM})$ or vehicle control $(0.1 \%$ [vol/vol] DMSO) for 30 minutes following 1 hour of serum deprivation. Cells were then either stimulated with $20 \mathrm{ng} / \mathrm{mL}$ EGF for 5 mins or left unstimulated (basal). Whole cell lysates were prepared for immunoblotting and probed with anti-phospho-Akt (S473) or anti-phospho-Src (Y416), and antiactin antibodies. Immunoblots were quantified as shown by mean $\pm \mathrm{SD}$ of respective antibody values, $\mathrm{p}<0.05$ relative to control (no vehicle) EGF-stimulated condition. 

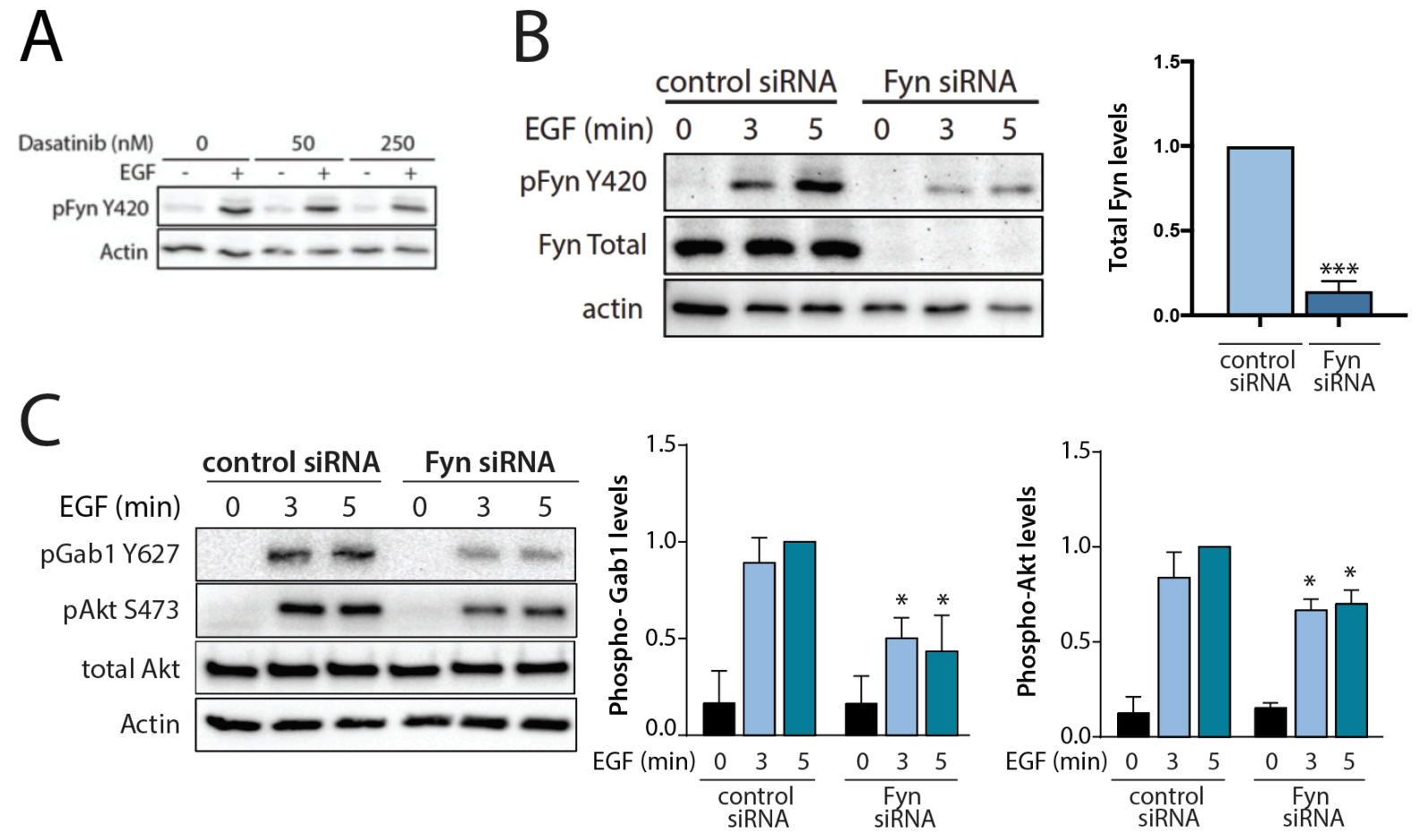

Figure 4.3 Fyn is required for EGF-stimulated Gab1 and Akt phosphorylation.

RPE cells were transfected with siRNA targeting Fyn or nontargeting siRNA (control), followed by serum deprivation for 1 hour and stimulation with $5 \mathrm{ng} / \mathrm{ml} \mathrm{EGF}$ for the time indicated (3-5 mins). Whole-cell lysates were resolved by immunoblotting and probed with antibodies as indicated. Shown are representative immunoblots of (A) phospho-Fyn (Y420), (B) phospho-Fyn (Y420) and total Fyn, (C) phospho-Gab1 (Y627), phospho-Akt (Y473), total Akt, and loading control (Actin) with respective quantification of phosphorylation intensity $\pm \mathrm{SE}$ (right panels); $\mathrm{n}$ $>3, * \mathrm{p}<0.05$ relative to the control siRNA-treated EGF stimulated conditions at each timepoint. 

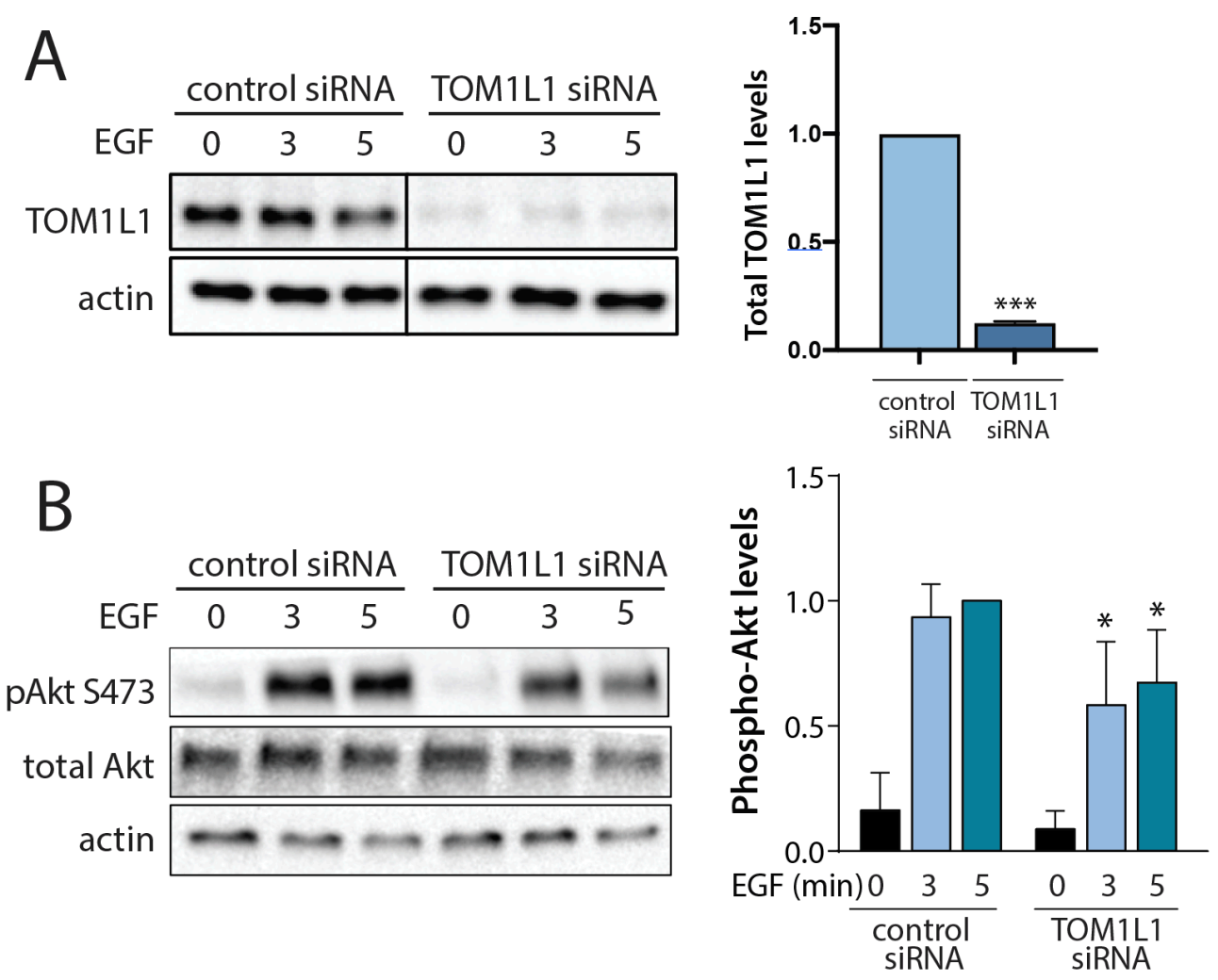

Figure 4.4 TOM1L1 is required for EGF-stimulated Akt phosphorylation.

RPE cells were transfected with siRNA targeting TOM1L1 or non-targeting siRNA (control). After transfection, cells were serum deprived for 1 hour followed by stimulation with $5 \mathrm{ng} / \mathrm{mL}$ EGF for 3 or 5 mins, as indicated. Whole-cell lysates were resolved by immunoblotting and probed with antibodies targeting (A) total TOM1L1, (B) phospho-Akt (S473), total Akt, or loading control (Actin). Shown are representative immunoblots (left panel) and mean phosphorylated Akt (pS473) $\pm \mathrm{SE}$ (right panels). $\mathrm{n}=3,{ }^{*} \mathrm{p}<0.05$ relative to the control siRNAtreated EGF-stimulated conditions at each timepoint. 


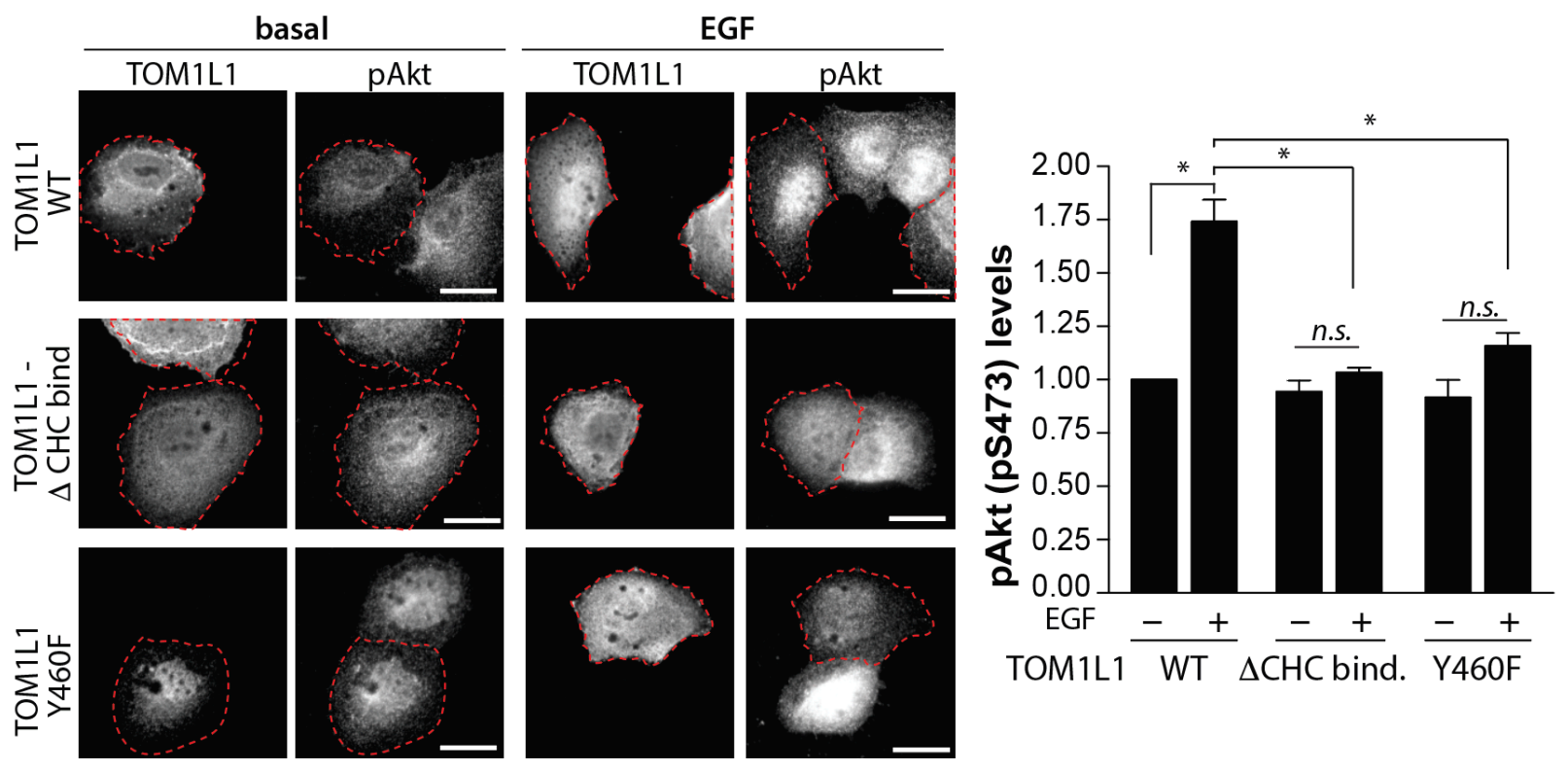

Figure 4.5 Fyn and clathrin binding by TOM1L1 is required for EGF-stimulated Akt phosphorylation.

RPE cells were transfected with either wildtype, clathrin-binding defective, or Y460F Fynbinding defective mutants of TOM1L1, followed by stimulation with $20 \mathrm{ng} / \mathrm{mL}$ EGF for 5 mins. Cells were then fixed and subjected to immunofluorescent staining of phosphorylated Akt (S473) and transfected TOM1L1 using an anti-HA antibody. Shown are representative micrographs (left panel), as well as the mean levels of phosphorylated Akt $\pm \mathrm{SE}$ in each condition (right panel). $\mathrm{n}>$ 60 cells from 3 independent experiments, $* \mathrm{p}<0.05$. Scale bar $=20 \mu \mathrm{m}$. 

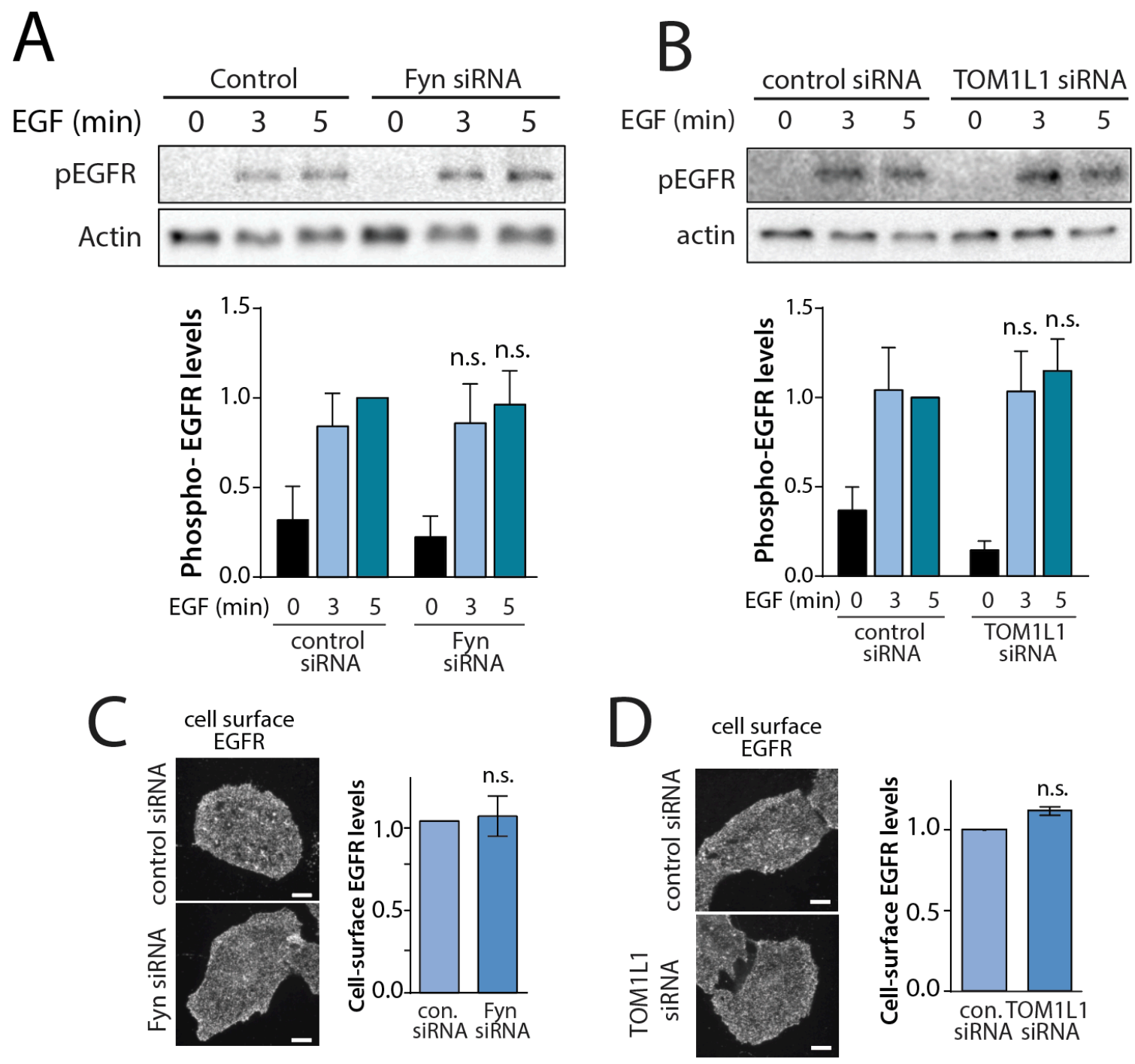

\section{Figure 4.6 EGFR is unaffected by Fyn or TOM1L1 siRNA.}

RPE cells were transfected with siRNA targeting Fyn, TOM1L1 or non-targeting siRNA (control). (A-B) After transfection, cells were serum deprived for $1 \mathrm{hr}$ followed by stimulation with $5 \mathrm{ng} / \mathrm{mL}$ EGF for 3 or 5 mins, or left unstimulated (basal). Whole-cell lysates prepared and resolved by immunoblotting and probed with anti-phospho-EGFR (Y1068) and anti-actin antibodies. Shown in (A-B) are representative immunoblots (top panels) and mean phosphorylated EGFR (Y1068) $\pm \mathrm{SE}$ (bottom panels). $\mathrm{n}=3,{ }^{*} \mathrm{p}<0.05$ relative to the control siRNA-treated EGF-stimulated conditions at each timepoint. (C-D) Following siRNA transfection, intact cells were subjected to immunofluorescence microscopy with antibodies that 
selectively recognize the EGFR ectodomain. Representative fluorescence microscopy micrographs of cell surface EGFR immunostaining are shown. Scale $=10 \mu \mathrm{m}$. Also shown are mean fluorescence intensity of cell surface EGFR \pm S.E. $(n=3)$. 


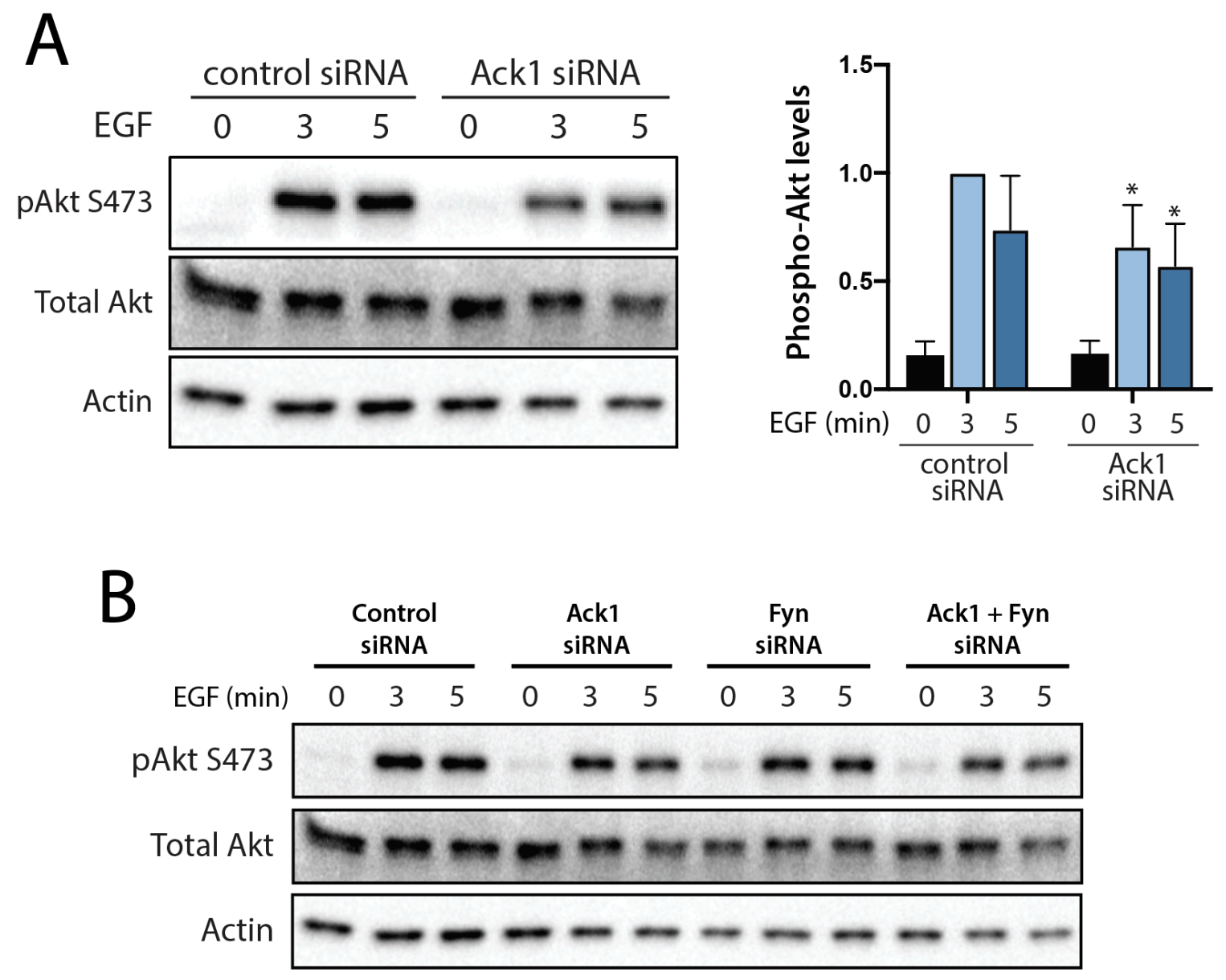

Figure 4.7 Ack1 is required for EGF-stimulated Akt phosphorylation.

(A) RPE cells were transfected with siRNA targeting Ack1 or non-targeting siRNA (control). After transfection, cells were serum deprived for $1 \mathrm{hr}$ followed by stimulation with $5 \mathrm{ng} / \mathrm{mL}$ EGF for 3 or 5 mins, as indicated. Whole-cell lysates were resolved by immunoblotting and probed with antibodies targeting phospho-Akt (S473), total Akt, or loading control (Actin). Shown are representative immunoblots (left panel) and mean phosphorylated Akt (pS473) $\pm \mathrm{SE}$ (right panels). $n=4, * p<0.05$ relative to the control siRNA-treated EGF-stimulated conditions at each timepoint. (B) RPE cells were transfected with siRNA targeting Ack1, Fyn, Ack1 and Fyn, or non-targeting siRNA (control). After transfection, cells were serum deprived for $1 \mathrm{hr}$ followed by stimulation with $5 \mathrm{ng} / \mathrm{mL}$ EGF for 3 or 5 mins, as indicated. Whole-cell lysates were resolved by immunoblotting and probed with antibodies targeting phospho-Akt (S473), total Akt, or loading control (Actin). 

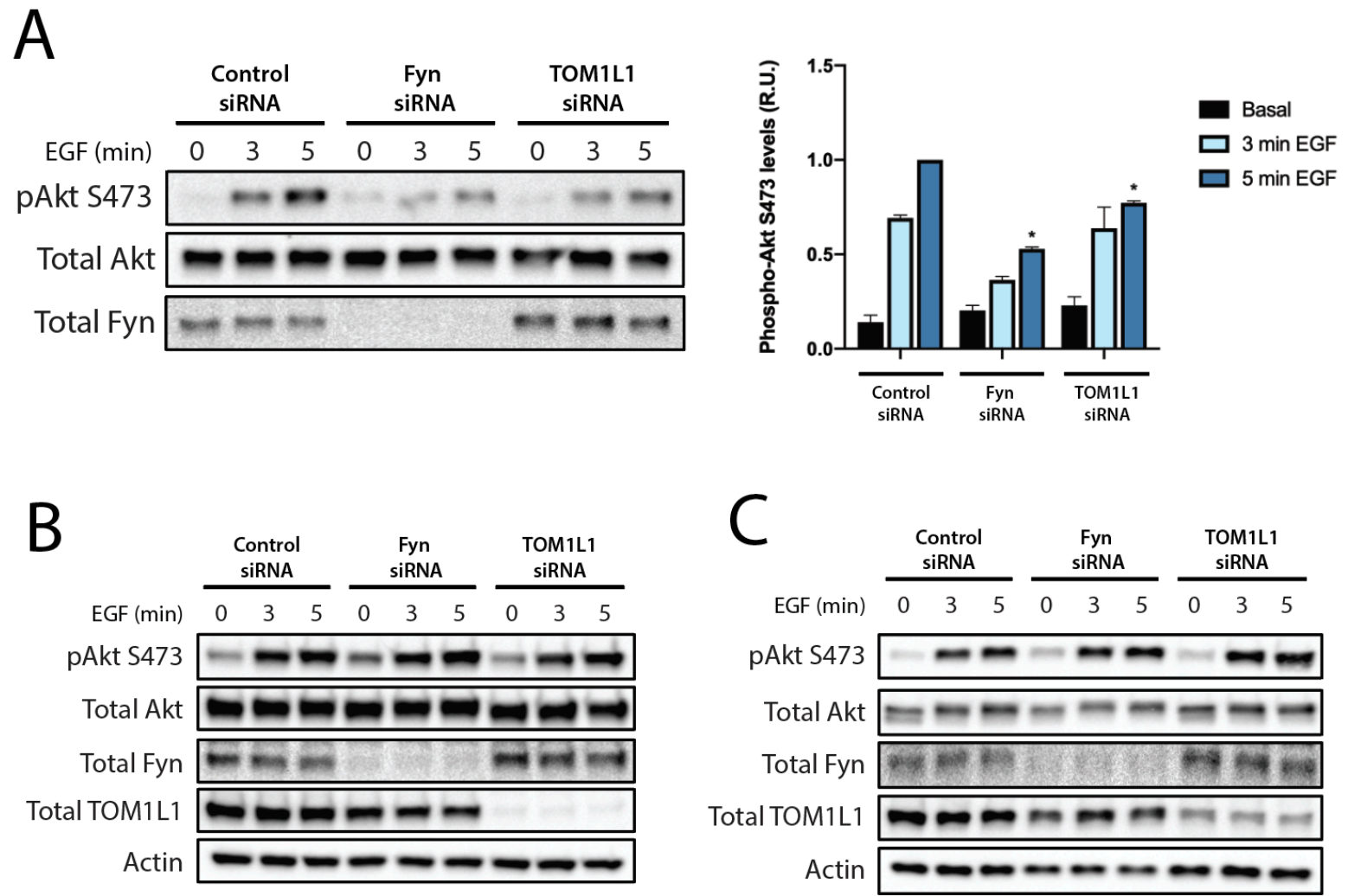

Figure 4.8 Fyn and TOM1L1 are required for MCF10A, but not SUM149PT or T47D cell EGF-stimulated Akt phosphorylation.

(A) MCF10A, (B) SUM149PT, or (C) T47D cells were transfected with siRNA targeting Fyn or TOM1L1, or non-targeting siRNA (control). After transfection, cells were serum deprived for 1 hr followed by stimulation with $5 \mathrm{ng} / \mathrm{mL}$ EGF for 3 or $5 \mathrm{mins}$, as indicated. Whole-cell lysates were resolved by immunoblotting and probed with antibodies targeting phospho-Akt (S473), total Akt, total TOM1L1, total Fyn, or loading control (actin). (A) Shown are representative immunoblots (left panel) and mean phosphorylated Akt (pS473) $\pm \mathrm{SE}$ (right panels). $\mathrm{n}=3, * \mathrm{p}<$ 0.05 relative to the control siRNA-treated EGF-stimulated conditions at each timepoint. 


\section{Chapter 5. A subset of clathrin-coated pits are unique signaling nanodomains required for EGFR signaling}

The plasma membrane is compartmentalized into a number of distinct nanodomains that allow context-specific control of cellular processes such as metabolism, migration, growth, and proliferation through spatial and temporal regulation of RTKs resident here. RTKs are recruited to and enriched within various plasma membrane nanodomains under diverse conditions, and facilitate the activation of many intracellular signal transduction pathways from here, including PI3K/Akt, Ras-MAPK, PLC, JAK-STAT, and Rho-family GTPases (Lemmon \& Schlessinger, 2010). In addition to EGFR, RTKs that undergo internalization via CME include TfR, Insulin receptor (Martins et al., 2011), RET receptor (Crupi et al., 2014), and the Met receptor (Hammond et al., 2001; Li et al., 2007). The CCPs that contain these receptors are as heterogeneous as the proteins found on the cell membrane, and these differences in cargo content also reflects heterogeneity in CCP behavior (Sousa et al., 2012; Merrifield et al., 2005; Mettlen et al., 2010b; Rappoport et al., 2005). Given this growing pool of literature defining CCPs as heterogeneous structures, the question still remains whether the signaling proteins activated downstream of RTK activation are recruited to CCPs at the plasma membrane, whether these CCPs recruit the same complement of proteins, and whether the CCPs that recruit these proteins are unique in any way. As I have shown that clathrin is required for signaling downstream of EGFR and Met receptor (Figure 3.1), acquired differences in recruited proteins or CCP dynamics may reflect the existence of a signaling-specific CCP subpopulation required to support RTK signaling.

In order to understand CME of RTKs quantitatively, we must track a large number of particles formed at the plasma membrane corresponding to a large number of CCPs in the cell at 
any given time (Liang et al., 2010). Manual tracking for large datasets like this is infeasible, therefore automatic tracking and analysis is important for quantitative studies. Over the past decade, the development of methods for the systematic detection, tracking and analysis of CCPs has allowed a new appreciation that most CCPs do not proceed along the textbook model of assembly that says each structure can lead only to the production of an intracellular vesicle (Aguet et al., 2013; Kadlecova et al., 2017; Mettlen \& Danuser, 2014). CCPs are generally smaller than $200 \mathrm{nM}$ in diameter, making them diffraction-limited objects that are below the limit of resolution of the light microscope (200 nM) (Lucarelli et al., 2017). Objects smaller than $200 \mathrm{nM}$ give rise to a light intensity distribution on camera known as an Airy pattern: a diffuse, delocalized and symmetric ring pattern of light with a radius of approximately $200 \mathrm{nM}$ (Zhang et al., 2007). The physical distance that a microscope requires to distinguish the individual light intensity pattern of each airy disk, or point spread function (PSF), is thus referred to as the limit of resolution in light microscopy (Zhang et al., 2007).

In order to obtain information about the diffraction limited object being imaged, modeling its PSF mathematically is required, which has been well described to be accurately approximated to a Gaussian function (Zhang et al., 2007). We can therefore detect CLSs based on the assumption that the fluorescent signal measured can be described by a Gaussian model of the PSF, where the amplitude of the Gaussian function reflects the fluorescence intensity of a CCP, which in turn informs about its size (Aguet et al., 2013; Garay et al., 2015; Lucarelli et al., 2017; Mettlen \& Danuser, 2014). From this analysis, we have learned that clathrin structures can be dynamically unstable, particularly at early stages of assembly, and that CCPs undergo abortive turnover at various stages in maturation resulting in short-lived subpopulations of nonproductive CCPs (Aguet et al., 2013; Kadlecova et al., 2017; Mettlen \& Danuser, 2014). In 
Chapter 5, I will couple Gaussian PSF modeling to both fixed and live cell imaging using Total Internal Reflection Fluorescence Microscopy (TIRF-M) to study CCPs enriched with RTKs and signaling regulators to delineate the formation of clathrin signaling nanodomains at the plasma membrane.

From this chapter, Figure 5.1 is published in Delos Santos et al., 2017.

From this chapter, Figure 5.4 is published in Lucarelli et al., 2017.

From this chapter, Figure $\mathbf{5 . 5}$ is published in Lucarelli et al., 2016.

\subsection{EGF and Transferrin are recruited to largely distinct CLSs}

EGFR and TfR are both internalized from the plasma membrane via CME, but unlike EGFR, internalization of TfR is largely unaffected by perturbation of AP2 (Motley et al., 2003) or changes in phospholipase $\mathrm{C} \gamma 1$ (PLC $\gamma 1)$, intracellular $\mathrm{Ca}^{2+}$, or protein kinase $\mathrm{C}$ (PKC) (R. Delos Santos et al., 2017). These differential requirements for endocytosis lead us to the hypothesis that these receptors reside in distinct populations of CCPs at the plasma membrane, which are able to recruit their own specific set of endocytic adaptor and accessory proteins. In order to directly and systematically determine if EGFR and TfR are indeed recruited to distinct CCPs, thus revealing the heterogeneity of specific clathrin structures, RPE cells stably expressing CLC fused to enhanced green fluorescent protein (eGFP) (eGFP-CLCa) were simultaneously labelled with fluorescently conjugated ligands of TfR and EGFR, A674Transferrin (Tfn) and rhodamine-EGF respectively. Ligands were added for 5 mins at various concentrations (as indicated in Figure 5.1), followed by fixation and then imaging by TIRF-M. 
This procedure allows the observation and quantification of diffraction-limited clathrin structures at the plasma membrane and their respective receptor content.

Here the term clathrin-labeled structures (CLSs) is used to describe clathrin structures observed or detected within single frame images in fixed samples using TIRF-M. Identification of bona fide CCPs requires additional analysis of CLS behavior over time that is only possible when examining live-cell images (Aguet et al., 2013; Kadlecova et al., 2017). CLSs can include CCPs as well as other clathrin structures that are sub-threshold which do not eventually give rise to bona fide CCPs, and may represent short-lived abortive structures that undergo rapid turnover (Aguet et al., 2013a; Kadlecova et al., 2017; Mettlen \& Danuser, 2014). As is visually apparent from the TIRF-M images in Figure 5.1A, CLSs harboring either EGFR (white circles) or TfR (purple circles) can be readily observed, while CLSs harboring both receptors at the same time are rarer, suggesting that TfR and EGFR are largely localized to distinct endocytic structures.

To quantitatively analyze the relationship between EGFR- or TfR-positive CLSs, TIRF$\mathrm{M}$ images were subject to automated detection and analysis, as has been established and thoroughly validated previously (Aguet et al., 2013b; Antonescu, et al., 2011; Garay et al., 2015; Jaqaman et al., 2008; Liu et al., 2010; Loerke et al., 2009; Mettlen et al., 2010). Importantly, this analysis allows the systematic, unbiased identification of all CLSs and measurement of fluorescence signal from receptors contained within them. A typical experiment examining $>15$ 20 cells per condition thus allows analysis and study of $>5000$ CLSs per condition, in turn allowing for a very robust study of differences in CLSs based on receptor content. The data of the intensity of each receptor type within CLSs was first plotted on a two-dimensional histogram as the distribution of fluorescent EGF versus fluorescent Tfn within all CLSs detected, which revealed an apparent mutually exclusive relationship between the receptor content of CLSs 
(Figure 5.1B). While the intensity of EGF or Tfn detected increases horizontally on each respective axis, the two populations of CLSs do not overlap, as would be exemplified by a linear relationship between the two ligand intensities on the histogram.

To directly quantify the proportion of CLSs that contain EGFR, TfR, or both receptors, the detected CLSs were sorted into cohorts by receptor content using an arbitrary but systematic cutoff value for classification of a CLS as positive for a receptor (Garay et al., 2015). This method of quantification showed that CLSs harboring EGF or Tfn were threefold more abundant than CLSs harboring both receptors when cells were treated with $20 \mathrm{ng} / \mathrm{mL}$ rhodamine-EGF and $10 \mathrm{ug} / \mathrm{mL}$ A647 Tfn (Figure 5.1C). This suggests that EGFR and TfR are indeed preferentially localized to distinct subpopulations of CLSs. However, this apparent separation of signals could be due to the incomplete engagement of EGFR with EGF at low, sub-saturating concentrations, such as the $20 \mathrm{ng} / \mathrm{mL}$ concentration used in these experiments. When cells were incubated with a higher concentration of EGF $(50 \mathrm{ng} / \mathrm{mL})$ while $\mathrm{Tfn}$ concentration was kept constant (10 $\mathrm{ug} / \mathrm{mL}$ ), the percentage of CLSs harboring EGF alone significantly increased while the percentage of CLSs containing both EGF and Tfn was unaltered (Figure 5.1C). This indicates that the preferential separation of EGFR and TfR into distinct subpopulations of CCPs is not due to incomplete engagement of the cell surface population of EGFR by EGF. Taken together, these data indicate that CLSs are heterogenous in nature, and that EGFR and TfR are found largely in distinct populations and thus may internalize through distinct classes or types of CCPs.

Imaging by TIRF-M takes advantage of the cell's attachment to the surface of a glass coverslip, allowing even illumination of fluorophores positioned at or near the plasma membrane on the ventral surface of the cell. Due to the polarized nature of most cells, events that occur on one side (i.e. the ventral side) may not be the same on the other side (i.e. the dorsal side). It has 
been reported that TfR internalization occurs largely on the ventral surface and EGFR internalization largely on the dorsal side (Grossier et al., 2014), although we can readily observe EGF labeling co-incident with CLSs on the ventral side as well, indicating that at least some EGFR internalizes on the ventral side. In order to determine if EGFR and TfR localization to distinct populations of CLSs may be specific to the ventral side of cell, EGFR and TfR labelling was repeated using their respective fluorescent ligands, this time with imaging by confocal microscopy, which allows separate imaging of the ventral and dorsal sides of the cell. In addition, since EGFR internalization is enhanced 10x by ligand binding, the potential overlap of EGFR and TfR into distinct CLS cohorts may be affected by the time component of EGF addition. In order to address this possibility, when this experiment was repeated, the EGF and TfR ligands were added for various time points ranging from 1 minute to 15 minutes of incubation (as indicated in Figure 5.1D-F), prior to imaging using spinning-disk confocal microscopy. These images were also subject to the same unbiased and systematic analysis of ligand recruitment to CLSs, this time as detected by AP2 staining (to allow detection of only cell surface clathrin structures) and across various z-sections of the cell.

As can be seen in representative micrographs, it is apparent that CLSs harboring either EGFR or TfR are readily observed across the ventral, middle, and dorsal sections (corresponding to the TIRF-M field, middle, and top of the cell), and that in each section, CLSs harboring both receptors are much less frequently observed (Figure 5.1D-F). CLSs were again also sorted into cohorts by ligand through automated detection and analysis, and quantification of the proportion of CLSs containing either receptor (but not both) revealed that they were threefold more abundant than CLSs harboring both receptors together on the ventral side of the cell (Figure 5.1D), consistent with the TIRF-M imaging and analysis done in Figure 5.1A-C. Analysis of the 
middle and top sections of cell revealed a substantial increase in the percentage of CLSs harboring EGFR, along with a decrease in the abundance of TfR CLSs. Additionally, CLSs containing both TfR and EGFR were virtually absent from these dorsal sections (Figure 5.1EF).

Taken together, this result indicates that TfR and EGFR reside largely in distinct populations of CLSs on the plasma membrane, and that this phenomenon is not dependent on receptor location on the plasma membrane of the cell. These observations reinforce the notion that CCPs at the cell surface are heterogeneous, and that distinct subpopulations of clathrin structures may have specific functions and are subject to specific forms of regulation. Given my results from Chapter 4 showing that various signaling proteins that can directly or indirectly bind clathrin (Fyn, Ack1) are required for clathrin-dependent signaling leading to Akt activation, this in turn raises several important questions: (i) are these signaling regulators indeed recruited to CLSs as the functional analysis may suggest and (ii) are the CLSs that recruit signaling regulators unique structures that form novel platforms for signal transduction?

\subsection{Endocytic accessory proteins and kinases are enriched within a subset of plasma membrane clathrin structures}

As determined in Chapter 4 through pharmacological inhibition and siRNA gene silencing, Fyn, TOM1L1, and Ack1 are all required for EGF-stimulated Akt phosphorylation, and this requirement phenocopies that of clathrin in EGFR signaling (Garay et al., 2015). In order to connect the activity of these proteins to signaling events downstream of EGFR activation, it is important to next determine their localization in the cell following EGF 
stimulation. Specifically, I here aim to determine whether Fyn, TOM1L1, Ack1, and Gab1 are located within or recruited to CCPs at the plasma membrane following activation of EGFR.

\subsubsection{Fyn is enriched within a subset of clathrin structures at the plasma membrane}

Fyn and all other SFKs are post-translationally myristoylated on a conserved glycine residue within the first 6 amino acid residues at the C-terminus, which aids in plasma membrane targeting as the lipid moiety is able to insert into the inner leaflet of the plasma membrane. In addition to this and relevant to RPE cells which only express three members of the SFKs (Src, Yes, and Fyn), Fyn alone is additionally palmitoylated on Cysteine 6 (Koegl et al., 1994; Paige et al., 1993) which can specifically target this kinase to membrane rafts. As a result of this, Fyn is found primarily in the membrane raft fraction in T-cells, and this localization has been proposed to be required for signaling downstream of the T-cell receptor aiding in T-cell activation (Ilangumaran et al., 1999; Palacios \& Weiss, 2004; Saito et al., 2010). Hence, Fyn may localize to either membrane rafts or clathrin structures in RPE cells.

To determine Fyn's localization in RPE cells, a plasmid encoding eGFP fused to Fyn (eGFP-Fyn) was transfected into RPE cells stably expressing Tag-RFP-T-tagged CLC (RPERFP-CLC) and stimulated with $20 \mathrm{ng} / \mathrm{mL}$ EGF for 5 minutes or left unstimulated (basal). Cells were then subject to imaging by TIRF-M to selectively illuminate the plasma membrane coupled to automated detection and analysis of CLSs. eGFP-Fyn exhibited little recruitment to clathrin structures in basal cells, however following stimulation with EGF, there was a noticeable enrichment of eGFP-Fyn within a subset of clathrin structures at the plasma membrane (Figure 5.2A). Automated detection and analysis of clathrin-labelled structures using the same Gaussian- 
based PSF modeling approach used in Chapter 5.1 to detect EGF and Tfn within CLSs (Aguet et al., 2013) revealed a statistically significant increase in eGFP-Fyn enrichment within CLSs following EGF stimulation (Figure 5.2A, right panel). Importantly, in any pairs of images representing two channels in which the detected structures are punctate and dense, it is expected that some overlap between the two signals may occur due to random proximity of the structures. To address this possibility, the analysis was repeated in pairs of images in which the position of the Fyn fluorescence channel relative to the clathrin channel was scrambled to randomize the position of Fyn structures relative to clathrin structures. Importantly, this randomization abolished the EGF-stimulated increase in eGFP-Fyn detected within CLSs, but not the apparent 'signal' of EGF within CLSs in the basal (non-EGF stimulated) state (Figure 5.2A, right panel). This indicates that the recruitment of eGFP-Fyn to CLSs upon EGF stimulation was specific and not an artifact of random overlap of unrelated dense objects within the image pairs. Given the similar levels of apparent eGFP-Fyn detected within CLSs in the basal state in the actual and randomized image pairs, it appears that there is little Fyn recruited to CLSs in the absence of EGF, and that most of the eGFP-Fyn signal in CLSs in the basal state is likely due to nonspecific signal overlap of eGFP-Fyn and CLSs.

Despite the comparison of real and randomized image pairs that show selective recruitment of Fyn to CLS, it remains possible that Fyn is specifically recruited to structures that are systematically adjacent to CLS and not specifically to CLS themselves. Distinguishing between these possibilities requires microscopy that can obtain better resolution of fluorescence distribution of Fyn and clathrin within these structures. Hence, to complement the results that I obtained with TIRF-M, we also performed structured illumination microscopy (SIM) to observe the localization of eGFP-Fyn and clathrin labelled structures at a higher resolution, since clathrin 
coated pits are in fact diffraction-limited structures and therefore below the resolution of the light microscope and TIRF-M. Briefly, SIM uses patterned illumination to excite the sample, where the position and orientation of the illumination light is changed multiple times, and the emitted sample fluorescence is recorded for each position (Gustafsson, 2000; Sochacki et al., 2017). Specialized algorithms are then able to use the raw data from each recorded position to reconstruct a final image that has a resolution of $100 \mathrm{nM}$ (as opposed to $200 \mathrm{nM}$ in conventional light microscopy such as TIRF-M) (Gustafsson, 2000). Using SIM, we observed that clathrin structures again exhibited partial overlap with eGFP-Fyn (Figure 5.2B). Line scan analysis of individual clathrin and Fyn structures revealed similar intensity profiles with similar peak fluorescence centers (Figure 5.2B, bottom panel), further supporting the specific recruitment of eGFP-Fyn to a subset of clathrin structures as seen using TIRF-M (Figure 5.2A). We therefore can conclude with confidence that Fyn is found within CLSs at the plasma membrane.

\subsubsection{TOM1L1 is enriched within a subset of clathrin structures at the plasma membrane}

TOM1L1 resides primarily within the cytoplasm in basal cells, and has been shown to become rapidly recruited to the plasma membrane following EGF stimulation, although the possible localization of TOM1L1 to clathrin structures at the plasma membrane had not been previously examined (Liu et al., 2009). Hence, it remains unclear where specifically within the plasma membrane TOM1L1 is recruited upon EGF stimulation. To determine the nanoscale localization of TOM1L1 within the plasma membrane of RPE cells, I again used a strategy of transfection with plasmid encoding eGFP fused to TOM1L1 (eGFP-TOM1L1) in RPE cells stably expressing RFP-CLC, followed by stimulation of samples with $20 \mathrm{ng} / \mathrm{mL}$ EGF for 5 
minutes, while others were left unstimulated (basal). These cells were fixed and imaged by TIRF-M followed by automated detection and analysis of CLSs. Using this approach, I

specifically selected the cells with the lowest detectable expression of TOM1L1 for imaging and analysis to ensure minimal potential for artifacts from overexpressed proteins. TOM1L1 was detected within CLSs in both the basal and EGF-stimulated conditions (Figure 5.3). Importantly, as was observed for the enrichment of Fyn within CLSs, the enrichment of TOM1L1 within CLSs was lost upon scrambling the relative position of the fluorescent channels, indicating that like Fyn, TOM1L1 enrichment within CLSs is specific and not an artifact of dense structures positive for clathrin and TOM1L1 within the plasma membrane. Taken together, these results indicate that both Fyn and TOM1L1 are recruited to CLSs, and that Fyn recruitment to CLSs is enhanced by EGF stimulation.

\subsubsection{Gab1 is enriched within a subset of clathrin structures at the plasma membrane}

Many EGFR-proximal signals such as phosphorylated Gab1 and Akt reach their peak activation within 1-3 minutes of ligand stimulation, a timeframe that coincides with receptor residence in CCPs (Kiyatkin et al., 2006; Sorkin \& Goh, 2009). I uncovered that phosphorylation of Gab1 and Akt requires clathrin but not receptor endocytosis, and that perturbation of Fyn, TOM1L1, or Ack1 significantly reduces phosphorylation of Akt following EGF stimulation (Figure 4.3, Figure 4.4, and Figure 4.7). The functional requirements for clathrin and clathrin structure localized proteins such as TOM1L1 and Fyn suggest that clathrin structures may be platforms for localized enrichment of signal transduction, or that clathrin and associated proteins may regulate signaling in an alternative manner. If the former, then I expect that phosphorylation 
of Gab1 may selectively occur within certain clathrin structures at the plasma membrane. However, it remains unknown whether phosphorylated Gabl is enriched within clathrin coated pits following ligand stimulation within this time frame.

To determine whether EGF stimulation elicits an enrichment of Gab1 within clathrin structures, RPE-RFP-CLC cells were stimulated with $20 \mathrm{ng} / \mathrm{mL}$ EGF for either 1, 3, or 5 minutes, or left unstimulated (basal) following $1 \mathrm{hr}$ of serum deprivation. Cells were then fixed and subject to immunofluorescence staining to detect phospho-Gab1 (Y627) and imaged using TIRF-M. As visualized in Figure 5.4, EGF stimulation elicits a robust increase in phosphorylated Gab1 visible within a subset of CCPs at the plasma membrane compared to basal cells. After just 1 minute of EGF stimulation, there is a maximal enrichment of Gab1 within CCPs; subsequent time points exhibit a subsequent decrease in phospho-Gab1 enrichment within CCPs.

Since HGF stimulation elicits a similar clathrin-dependent increase in Gab1 phosphorylation as does EGF (Figure 3.4), I next aimed to determine whether HGF stimulation also elicits enrichment of phospho-Gab1 (Y627) in a subset of CCPs at the plasma membrane. RPE cells stably expressing RFP-CLC were stimulated with $50 \mathrm{ng} / \mathrm{mL}$ HGF and subject to immunofluorescence staining to detect phospho-Gab1 (Y627), as I had done for experiments shown in Figure 5.4 in EGF-stimulated cells. Cells were then imaged using TIRF-M followed by detection and analysis of CLSs, which allowed measurement of phospho-Gab1 within these structures. Similarly to what I observed upon EGF stimulation, HGF stimulation elicited an increase in phospho-Gab1 (Y627) enrichment within CLSs at the plasma membrane (Figure 5.5). Importantly, randomizing the position of the clathrin and phospho-Gab1 images ablated the HGF-stimulated increase in phospho-Gab1 enrichment within CLSs. This shows that the increase 
detected by automated detection and analysis was specific, and not due to random overlap of puncta in either image set. Taken together, this data indicates that phospho-Gab1 is enriched within a subset of clathrin structures at the plasma membrane following stimulation with either EGF or HGF, and exhibits its maximal enrichment peak quite early (1 minute) following ligand stimulation with EGF.

\subsubsection{Ack1 is enriched within a subset of clathrin structures at the plasma membrane}

Ack1 is able to directly interact with clathrin, and when overexpressed has previously been found within CCPs, on intracellular CCVs, and on endosomes colocalized with clathrin (Grøvdal et al., 2008; Shen et al., 2011). In order to determine if Ack1 specifically localizes to clathrin coated pits on the plasma membrane, RPE cells stably expressing RFP-CLC were transfected with plasmid encoding eGFP tagged to Ack1 (eGFP-Ack1) and stimulated with 20 ng/mL EGF or left unstimulated (basal). As with Fyn and TOM1L1 (Figure 5.2 and Figure 5.3), cells expressing the lowest possible detectable level of GFP-Ack1 were chosen for imaging using TIRF-M, and this was coupled to automated detection and analysis of CLSs. Stimulation of RPE cells with EGF resulted in a significant enrichment of eGFP-Ack1 within CLSs at the plasma membrane compared to basal (unstimulated) (Figure 5.6). This suggests that along with Fyn, TOM1L1 and Gab1, Ack1 is recruited to a subset of CLSs that may be specialized for signaling functions. 


\subsection{TOM1L1 is required for recruitment of Fyn to clathrin structures}

As previously mentioned, TOM1L1 is a signaling regulator and adaptor protein that is able to bind and activate Fyn (Seykora et al., 2002). TOM1L1 interacts with the SH2 domain of Fyn via a motif that includes phosphorylated Y460 (on TOM1L1), and is also able to bind the SH3 domain of Fyn via a RLP421 PLP motif (Li et al., 2005). Perturbation of either of these motifs on TOM1L1 substantially impaired the interaction of TOM1L1 with Fyn (Li et al., 2005). Through these interactions as well as the ability of TOM1L1 to bind clathrin and its enrichment within CLSs at the plasma membrane, TOM1L1 may be responsible for Fyn's recruitment to clathrin structures since Fyn is unable to directly bind clathrin or EGFR. To determine the possible relationship between recruitment of TOM1L1 and Fyn to CLSs, TOM1L1 was silenced using siRNA and RPE cells were transfected with a plasmid encoding eGFP-Fyn. Following this, cells were stimulated with $5 \mathrm{ng} / \mathrm{mL}$ EGF for 5 mins, fixed, and imaged using TIRF-M coupled to automated detection and analysis of CLSs. As I did previously, cells expressing the lowest possible detectable levels of eGFP-Fyn were selected for imaging and analysis to limit possible artifacts from protein overexpression. In cells treated with non-targeting siRNA, stimulation with EGF resulted in a significant increase in eGFP-Fyn detected within CLSs at the plasma membrane (Figure 5.7). In contrast, in cells treated with TOM1L1 siRNA, EGF stimulation was unable to elicit an increase in eGFP-Fyn enrichment within CLSs. These results indicate that TOM1L1 is required for the EGF-stimulated recruitment of Fyn to plasma membrane CLSs. 


\subsection{Fyn and Ack1 are recruited to a subset of EGF-positive clathrin structures}

While the enrichment of Fyn within CLSs following EGF-stimulation was specific and significant, the overall gain in recruitment to clathrin structures was only increased by approximately $50 \%$ when examining overall ensemble average (Figure 5.2A, Figure 5.7). This suggests that either low levels of Fyn are recruited broadly to a large fraction of CLSs, or that they may be selectively and more potently recruited to a smaller subset of CLSs. EGFR is recruited to a subset of CLSs that is largely distinct from those that harbor TfR (Figure 5.1). This suggests that CCPs are heterogeneous in nature, and that Fyn may be preferentially enriched within a subset of CCPs that harbor EGFR, allowing them to regulate receptor-proximal signaling.

To examine this possibility, RPE cells were again transfected with the lowest detectable levels of eGFP-Fyn, followed by stimulation with rhodamine-conjugated EGF for 5 mins and detection of CLSs by immunofluorescence staining to detect the clathrin adaptor AP2 (Figure 5.8), followed by imaging using TIRF-M. Automated detection and analysis of the images obtained was used to determine the distribution of eGFP-Fyn within CLSs in the EGF-stimulated condition. As observed in Figure 5.2 and Figure 5.7, EGF-stimulation caused a significant increase in eGFP-Fyn within CLSs (Figure 5.8B). The frequency distribution of eGFP-Fyn intensities detected within CLSs revealed that the majority of CLSs had little or no eGFP-Fyn enrichment in both the basal and EGF-stimulated conditions, and that EGF-stimulation elicited a specific increase in the appearance of CLSs with higher levels of eGFP-Fyn enrichment (Figure 5.8C). This suggests that EGF stimulation causes the recruitment and enrichment of eGFP-Fyn to a specific subset of CLSs at the plasma membrane. 
To determine if eGFP-Fyn recruitment occurs preferentially to the subset of CLSs that also contain EGFR, CLSs were sorted into EGF-positive and EGF-negative cohorts based on rhodamine-EGF residence with an arbitrary but systematic threshold for intensity, as previously done (Delos Santos et al., 2017; Lucarelli et al., 2017). The cohort of EGF-positive CLSs exhibited significantly more eGFP-Fyn enrichment than did the EGF-negative CLS cohort (Figure 5.8D). The frequency distribution plot revealed that CLSs with higher levels of eGFPFyn were more often detected in the EGF-positive cohort over the EGF-negative cohort (Figure 5.8E). CLSs were then also sorted and classified by Fyn status, as was done for EGF. This analysis revealed that EGF-positive/Fyn-positive CLSs were significantly more abundant following EGF stimulation than EGF-negative/Fyn-positive CLSs (Figure 5.8F). Together these results show that Fyn is recruited to a subset of CLSs at the plasma membrane, that Fyn recruitment occurs preferentially to the subset of CLSs that also harbor EGFR, and that following EGF stimulation, there are more EGF-positive CLSs that contain Fyn than those that do not.

Since Ack1 was also found to be required for EGF-stimulated Akt phosphorylation, and localizes to a subset of CLSs at the plasma membrane following EGF-stimulation, I next aimed to determine whether similarly to Fyn, Ack1 becomes preferentially recruited to CLSs containing EGFR. RPE cells were transfected with the lowest detectable levels of eGFP-Ack1, followed by stimulation with Alexa555-conjugated EGF or Alexa555-conjugated Tfn for 3 mins to label EGFR and TfR respectively. Cells were then fixed and immunofluorescently labelled with an anti-clathrin antibody to detect all CLSs, and images using TIRF-M. Images were subjected to automated detection of clathrin structures (as per Garay et al. 2015) followed by calculation of a correlation score between Ack1-EGF or Ack1-Tfn fluorescence within each structure. As 
quantified in Figure 5.9, Ack1 was found to localize preferentially to EGF-labelled CLSs and not Tfn-labelled CLSs. This finding demonstrates that, along with Fyn, Ack1 recruitment occurs preferentially to the subset of CLSs that also harbor EGFR, and may therefore be regulating signaling downstream of the receptor here.

\subsection{Fyn- or TOM1L1-positive CCPs have unique lifetimes and size.}

Thus far in Chapter 5, I have examined the recruitment of TOM1L1, Fyn and Ack1 to a specific subset of clathrin structures at the plasma membrane. The selective recruitment of Fyn to specific clathrin structures may reflect the random recruitment of Fyn to a small number of clathrin structures or may instead reflect the selective recruitment of Fyn to defined clathrin structures with defined properties and composition. To further understand how Fyn positive CCPs may be unique clathrin structures specialized in regulating signaling from the plasma membrane, I next examined the lifetime and size of Fyn-positive versus Fyn-negative structures. CCPs exhibit substantial heterogeneity in lifetimes and size (Aguet et al., 2013; Mettlen and Danuser, 2014; Kadlecova et al., 2017), as well as protein composition. eGFP-Fyn was transfected into RPE cells stably expressing Tag-RFP-T-tagged clathrin light chain (RPE-RFPCLC), and cells expressing the lowest possible detectable level of eGFP-Fyn were chosen for live cell imaging using TIRF-M following EGF stimulation. Representative single image frames taken from a sample time lapse image series of Fyn are shown in Figure 5.10A. A representative time lapse of a Fyn positive CCP showing fluorescence images corresponding to clathrin and Fyn centered at the detected object (top panels), as well as quantification of eGFP-Fyn and RFPCLC within the object (bottom panels) is shown in Figure 5.10B. 
Fyn was detected in CCPs over a broad range of lifetimes, and strikingly, the lifetime distribution of Fyn-positive CCPs is heavily skewed towards longer lifetimes compared to that of Fyn-negative CCPs (Figure 5.10C). The mean level of eGFP-Fyn detected over the entire lifetime of relatively short-lived (10-20s and 20-40s) CCPs was significantly higher than that recruited to longer-lived CCPs (Figure 5.10D). Hence, in contrast to clathrin and AP2, which exhibit recruitment to CCPs in a manner that scales with CCP lifetime (Loerke et al., 2011), the average Fyn recruitment level to CCPs does not scale with CCP lifetime and instead occurs to a higher extent in short-lived CCPs. Of note is the efficient sorting of Fyn-positive and Fynnegative CCPs, as determined by the mean eGFP-Fyn levels detected in each CCP cohort (Figure 5.10D). These data collectively indicate that Fyn is recruited to a unique subset of CCPs with distinct, elevated lifetimes, and that the extent of Fyn recruitment to CCPs is not a direct reflection of the levels of structural proteins such as clathrin or AP2. Fyn may thus be recruited throughout the lifetime of specific CCPs, raising the question of whether Fyn positive CCPs exhibit unique properties at the earliest stages of CCP assembly.

To determine if the unique lifetime distribution of Fyn-positive CCPs indeed reflects distinct assembly properties, we examined the size of CCPs, which depends on the rate of assembly of clathrin and occurs within the early stages of CCP formation (Loerke et al., 2011). We examined the mean plateau level of clathrin (clathrin intensity spanning the time following the initial burst of assembly to that preceding disassembly/fission) in cohorts of CCPs grouped by similar lifetimes, since CCP size scales with lifetime (Loerke et al., 2011). We found that across all lifetime cohort groupings, Fyn-positive CCPs exhibit higher levels of mean clathrin fluorescence intensity and were thus larger compared to Fyn-negative CCPs (Figure 5.10E). Importantly, the longer-lived and larger CCPs that are eGFP-Fyn positive were unlikely a 
consequence of expression of exogenous eGFP-Fyn, as the lifetimes and size of all CCPs (i.e. not sorted by Fyn status) were identical in cells expressing eGFP-Fyn as in untransfected cells imaged in parallel (Figure A5). This is likely a result of selecting cells expressing the lowest possible detectable level of eGFP-Fyn for these experiments. Taken together, these results indicate that Fyn is recruited to a subset of CCPs that exhibit unique lifetime and clathrin assembly (the latter being reflected as larger CCP size), which further indicates that Fyn becomes enriched in a specific subset of CCPs with distinct properties.

Using a similar live cell imaging approach, we observed that eGFP-TOM1L1 was similarly recruited to a subset $(8.4 \pm 0.6 \%)$ of CCPs (Figure 5.10F-G). Importantly, the CCPs positive for eGFP-TOM1L1 exhibited higher lifetimes than eGFP-TOM1L1-negative CCPs (Figure 5.10H), eGFP-TOM1L1 was detected at higher levels on average in short lived CCPs (Figure 5.10I), and CCPs that contained eGFP-TOM1L1 were larger than those without eGFPTOM1L1 (Figure 5.10J), similarly as was observed for CCPs that exhibit Fyn recruitment (Figure 5.10C-D). As with Fyn, these results indicate that TOM1L1 is recruited to a subset of CCPs that exhibit unique lifetime and clathrin assembly which again indicates that TOM1L1 is enriched within a subset of CCPs following EGF stimulation, and that this subset of CCPs exhibits distinct properties from TOM1L1-negative CCPs.

\subsection{Fyn-positive clathrin structures contain a unique profile of endocytic accessory proteins.}

The recruitment of Fyn and TOM1L1 to subsets of CCPs with distinct dynamic behavior suggests that Fyn recruitment to CCPs may be a novel component of the compositional 
heterogeneity of CCPs. Fyn-positive, signaling-capable clathrin structures may either (i) have a dramatically distinct compositional profile, such that Fyn positive CCPs could be devoid of or over-endowed with specific endocytic accessory proteins, (ii) have modest alterations in recruitment of specific endocytic proteins, indicating a combinatorial impact of the unique recruitment of certain proteins on the unique lifetime and size of signaling-capable CCPs, or (iii) be indistinguishable from other CCPs in terms of endocytic protein recruitment, suggesting that other factors drive the unique CCP lifetime, size and function of these signaling structures. To determine whether the endocytic protein composition of CCPs differs in Fyn positive signalingcapable CCPs, we quantified the recruitment of three curvature-generating proteins involved in CME to Fyn-positive versus Fyn-negative clathrin structures: epsin, endophilin and amphiphysin.

To do so, we used immunofluorescence staining and TIRF-M to detect the endogenous localization of each of these endocytic accessory proteins to CLSs in cells expressing RFP-CLC and the lowest detectable levels of transfected eGFP-Fyn (Figure 5.11). As expected, epsin, endophilin and amphiphysin were readily detected within a subset of CLSs at the plasma membrane, including in structures that were also positive for eGFP-Fyn. Following imaging, automated detection and analysis of these CLSs to sort detected structures into Fyn-positive and Fyn-negative cohorts was performed. Since the extent of recruitment of endocytic proteins may depend on size and lifetime of clathrin structures, the level of each of epsin, endophilin or amphiphysin was measured as a ratio to the level of AP2 detected in each structure. Using this approach, epsin was modestly but significantly less abundant in Fyn-positive than in Fynnegative CLSs (Figure 5.11A). In contrast, endophilin was equally recruited to Fyn-positive and Fyn-negative CLSs (Figure 5.11B), and amphiphysin was recruited modestly but significantly 
more to Fyn-positive than Fyn-negative CLSs (Figure 5.11C). The unique recruitment of curvature-generating proteins to structures harboring eGFP-Fyn did not result in gross changes in curvature generation in these structures (Figure A5C-D). These results indicate that Fyn-positive CCPs may exhibit a collection of modest but discernable differences in endocytic protein recruitment compared to Fyn-negative structures. As such, Fyn-positive, signaling-capable clathrin structures are not only distinct in terms of size and lifetime, but also exhibit unique compositional profile(s) relative to Fyn-negative clathrin structures.

\subsection{Chapter 5 Summary}

This chapter outlines a role for CCPs as a type of signaling nanodomain required for the potentiation of EGFR signaling downstream of ligand activation, adding to a growing pool of literature that is radically shifting our understanding of CCPs from the endocytic portals they were once solely thought to be. Using TIRF-M coupled to automated detection and analysis of clathrin structures, I determined that EGFR and TfR are largely resident within distinct pools of CCPs at the plasma membrane, and that only a small percentage of CCPs contain both receptors at the same time. It next followed to examine the recruitment of signaling intermediates downstream of EGFR to CCPs, and determine whether these proteins were broadly recruited to all CCPs or whether their enrichment was concentrated in a subset of structures. I found that EGF stimulation elicits the enrichment of Fyn within a subset of clathrin structures at the plasma membrane in a TOM1L1-depdent manner, as perturbation of TOM1L1 using siRNA ablated Fyn's enrichment within CCPs. I also determined that phospho-Gablis enriched within a subset of clathrin structures at the plasma membrane following stimulation with either EGF or HGF. Additionally, Ack1 is found within a subset of clathrin structures that preferentially contain 
EGFR following EGF-stimulation, and TOM1L1 is also found within a subset of clathrin structures, however this enrichment is independent of EGF stimulation.

I hence propose that the term "clathrin signalosome" be used to describe Fyn, Ack1 and/or TOM1L1 positive CCPs that are required for EGFR signaling to activate the PI3K/Akt pathway. To further characterize these clathrin signalosomes, I examined the unique endocytic accessory protein composition of clathrin structures that contain Fyn. The curvature-inducing protein Epsin was found to be significantly less abundant in Fyn-positive clathrin structures, and an additional curvature-inducing endocytic protein Amphiphysin was found to be significantly more abundant in Fyn-positive clathrin structures. I also find that Fyn or TOM1L1 positive clathrin structures have unique lifetimes and size compared to clathrin structures devoid of either.

These unique characteristics of clathrin structures that contain Fyn or TOM1L1 when taken together suggest that clathrin may be coordinating the recruitment of a specific set of endocytic effector proteins to create signaling nanodomains required for the propagation of EGFR signaling downstream. Clathrin may therefore be acting as a signaling scaffolding protein, coordinating the signaling downstream of receptors resident within a subset of CCPs at the plasma membrane. I propose that TOM1L1 and Fyn define a unique subset of signaling-capable plasma membrane clathrin structures required for specific facets of EGFR signaling leading to Akt activation. 


\subsection{Chapter 5 Figures}

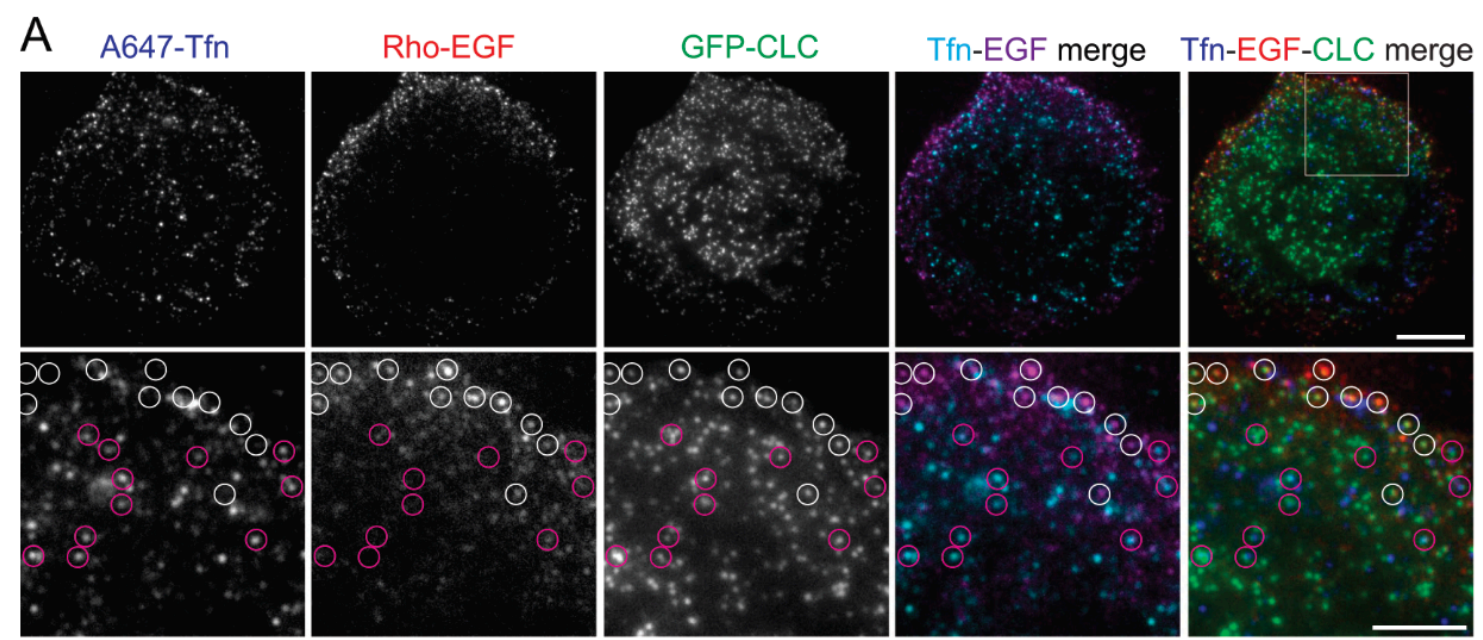

B

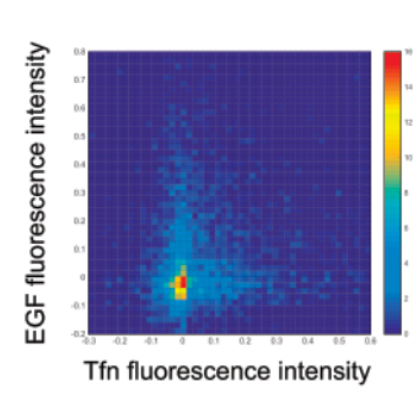

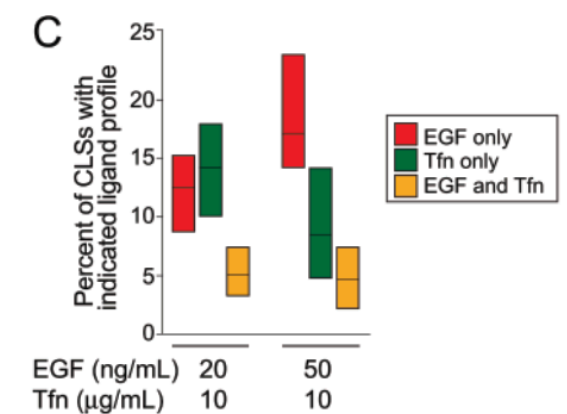

E

$\mathrm{F}$
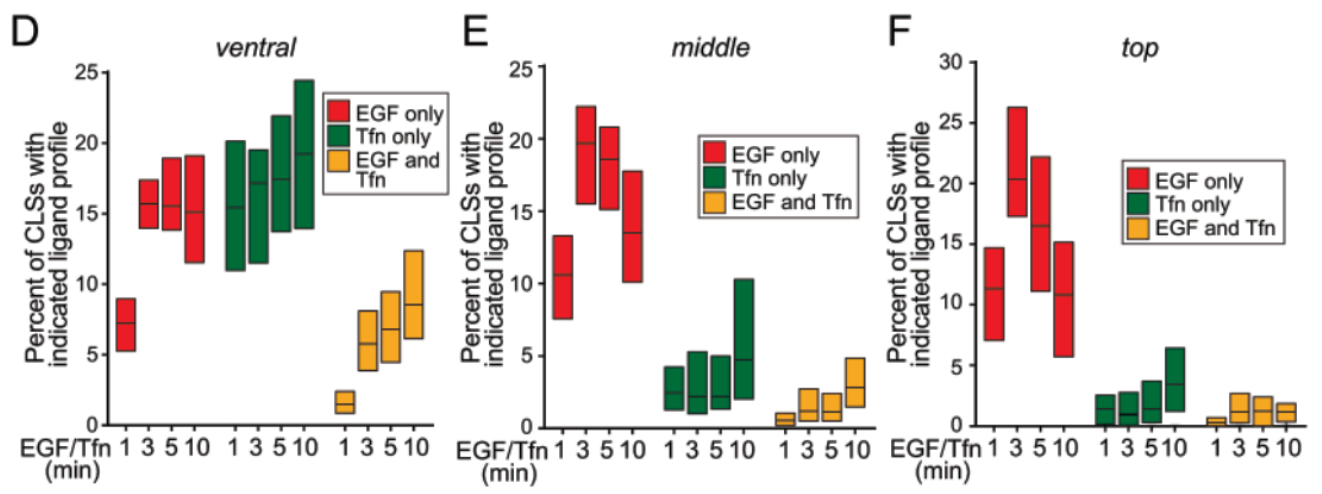

Figure 5.1 EGFR and Tfn are recruited largely to distinct clathrin structures.

RPE cells stably expressing clathrin light chain fused to eGFP (eGFP-CLCa) were treated with 20 $\mathrm{ng} / \mathrm{ml}$ rhodamine-EGF (Rho-EGF) and $10 \mu \mathrm{g} / \mathrm{ml} \mathrm{A647-Tfn} \mathrm{for} 5 \mathrm{~min}$ or the indicated time, followed by immediate fixation. (A) Shown are representative images obtained by TIRF-M. Scale bars: $10 \mu \mathrm{m}$ (top row); $5 \mu \mathrm{m}$ (bottom row, corresponding to enlarged images of the region 
shown in the merged image of the top row). White circles depict CLSs that are positive for EGF but devoid of Tfn, and purple circles depict CLSs that are positive for Tfn but not EGF. (B, C) CLSs were subjected to automated detection and analysis as described in Materials and Methods. Shown in (B) is a two-dimensional histogram of the normalized EGF and Tfn fluorescence intensities in each CLS cohort. Shown in (C) are median (bar) and 25th/75th percentiles (boxes) of the proportions of CLSs that are positive for EGF (but not Tfn), Tfn (but not EGF), or both EGF and Tfn. The number of CLSs and cells analyzed, respectively, for each condition are as follows: $20 \mathrm{ng} / \mathrm{ml}$ rhodamine-EGF and $10 \mu \mathrm{g} / \mathrm{ml} \mathrm{A647-Tfn:} \mathrm{70,124} \mathrm{and} \mathrm{57;} \mathrm{and} 50 \mathrm{ng} / \mathrm{ml}$ rhodamine-EGF and $10 \mu \mathrm{g} / \mathrm{ml}$ A647-Tfn: 46, 240 and 37; from a minimum of three independent experiments in each condition. (D-F) Images obtained by spinning-disk confocal microscopy, corresponding to ventral, middle, and top z-sections of cells were subjected to automated detection and analysis as described in Materials and Methods. Shown are median (bar) and 25th/75th percentiles (boxes) of the proportions of CLSs in the ventral (D), middle (E), and top (F) z-sections that are positive for EGF (but not Tfn), Tfn (but not EGF), or both EGF and Tfn. 

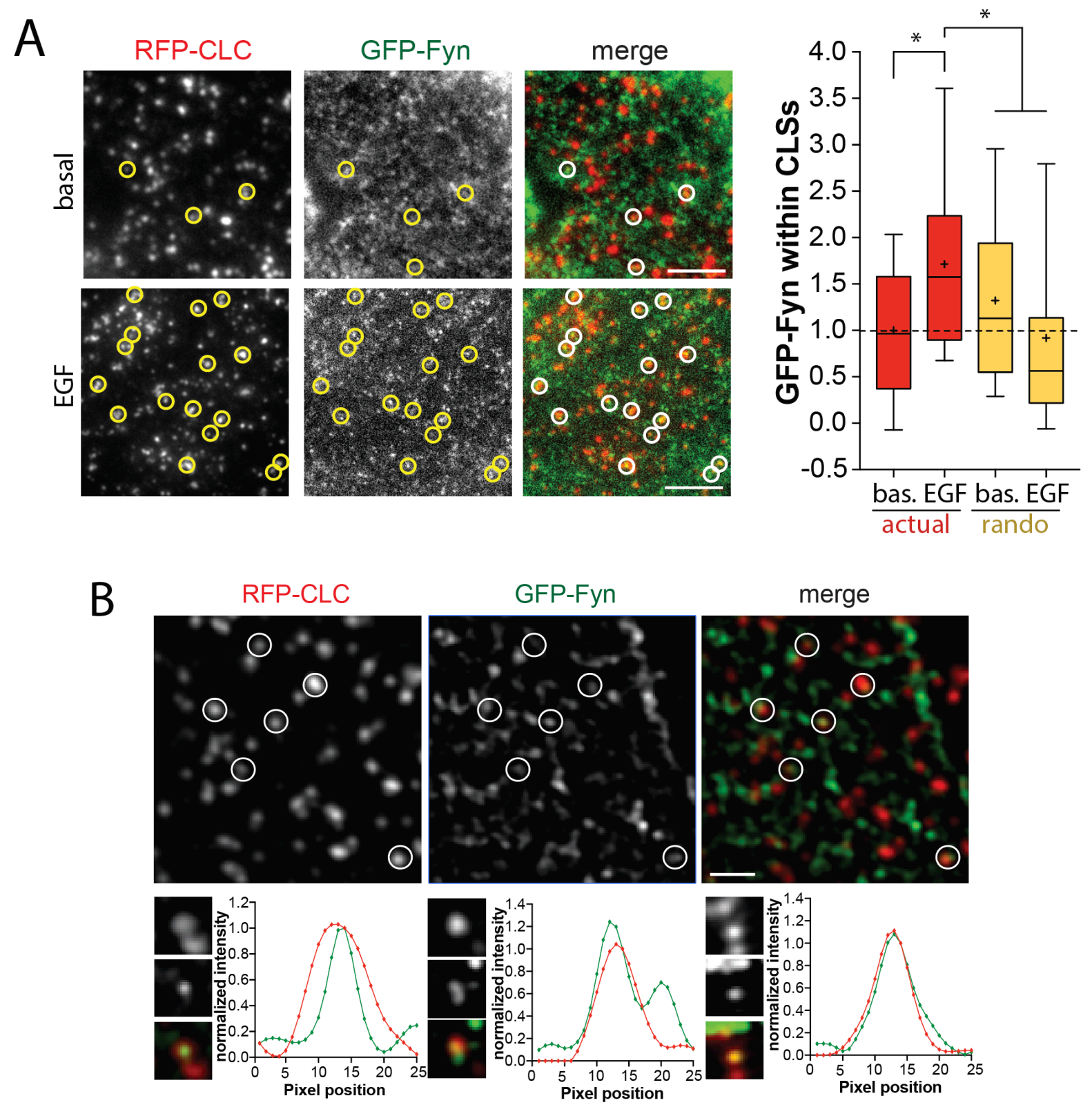

Figure 5.2 Fyn is enriched within a subset of plasma membrane clathrin structures.

RPE cells stably expressing RFP fused to clathrin light chain (Tag-RFP-T-CLC) were transfected eGFP fused to Fyn (GFP-Fyn). (A) Cells were stimulated with 20 ng/mL EGF for 5 min or left unstimulated (basal), then fixed and imaged using TIRF-M. Shown (left panel) are representative micrographs depicting Fyn-positive clathrin structures (circles), identified manually. Scale $=5$ 
$\mu \mathrm{m}$. Full size image panels obtained by TIRF-M are available in Figure A2A. Micrographs obtained by TIRF-M were subjected to automated detection and analysis of clathrin structures, allowing quantification of eGFP-Fyn and RFP-CLC in each detected object. Shown (right panel) is the measurements of eGFP-Fyn fluorescence intensity within CLSs, depicted as median (bar) 25 th $/ 75^{\text {th }}$ percentiles (boxes) and whiskers (full range). We report measurements performed in actual Fyn and CLC image pairs ('actual'), as well as in the same image pairs in which one of the channels was rotated 180 degrees to randomize the position of Fyn structures relative to clathrin structures ('rando'). The number of CLSs and cells analyzed are as follows: eGFP-Fyn expression, basal (44 cells, 6641 CLSs), EGF stimulated (31 cells, 6300 CLSs), *, $p<0.05$. (B) RPE cells transfected to express eGFP-Fyn were subject to Structured Illumination Microscopy (SIM). Shown are representative micrographs (top panels) and line scan analysis of AP2 and eGFP-Fyn in individual clathrin structures (bottom panels). scale $=1 \mu \mathrm{m}$. Full size image panels obtained by SIM are available in Figure A3. This was done in collaboration with the Kim lab at University of Toronto / Peter Gilgan Center for Research and Learning. 

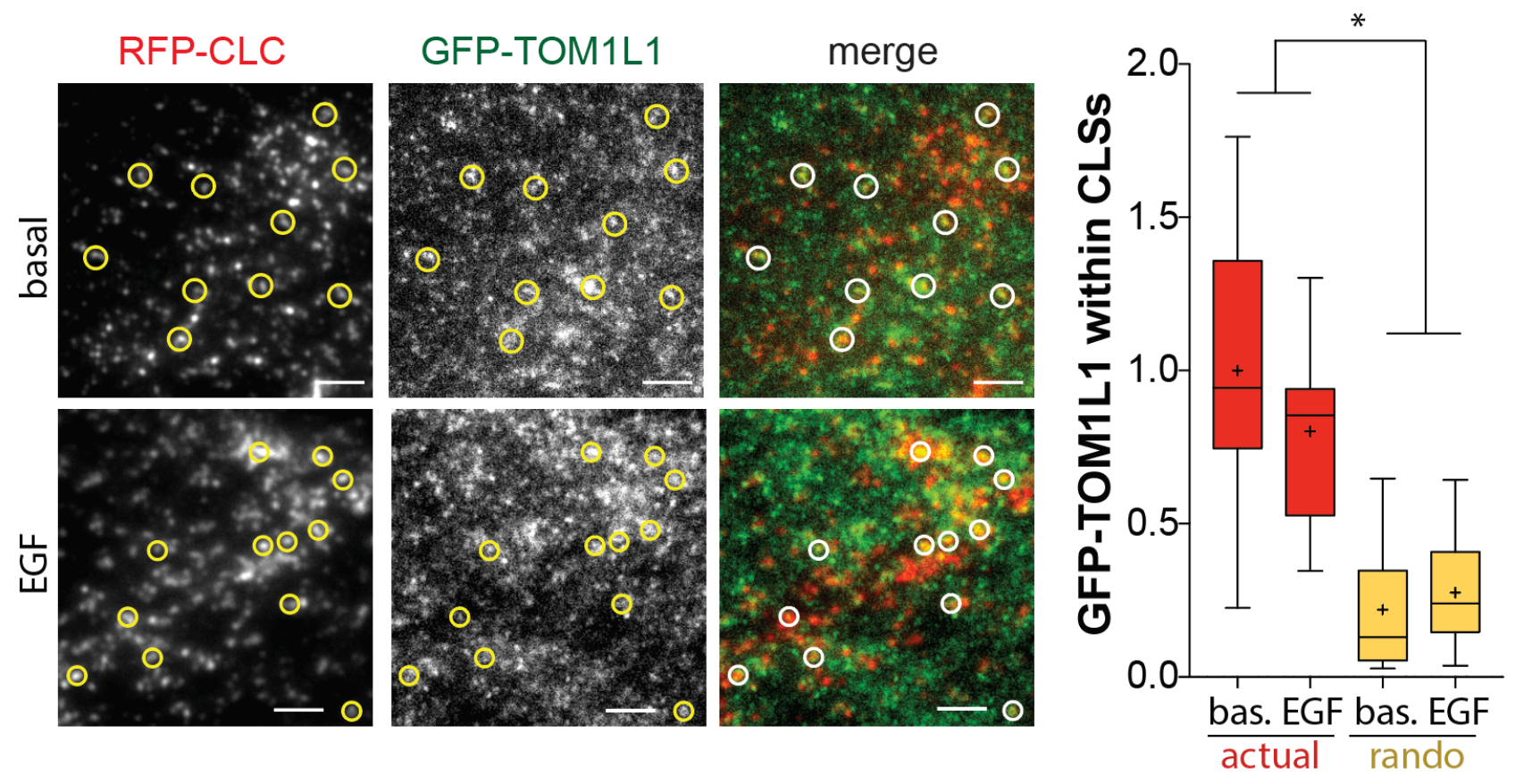

Figure 5.3 TOM1L1 is enriched within a subset of plasma membrane clathrin structures.

RPE cells stably expressing RFP fused to clathrin light chain (Tag-RFP-T-CLC) were transfected with a plasmid encoding eGFP fused to TOM1L1 (GFP-TOM1L1). Cells were stimulated with $20 \mathrm{ng} / \mathrm{mL}$ EGF for $5 \mathrm{~min}$ or left unstimulated (basal), then fixed and imaged using TIRF-M. Shown (left panel) are representative micrographs depicting TOM1L1-positive clathrin structures (circles), identified manually. Scale $=5 \mu \mathrm{m}$. Full size image panels obtained by TIRFM are available in Figure A2B. Micrographs obtained by TIRF-M were subjected to automated detection and analysis of clathrin structures, allowing quantification of eGFP-TOM1L1 and RFPCLC in each detected object. Shown (right panel) is the measurements of eGFP-TOM1L1 fluorescence intensity within CLSs, depicted as median (bar) $25 \mathrm{th} / 75^{\text {th }}$ percentiles (boxes) and whiskers (full range). We report measurements performed in actual TOM1L1 and CLC image pairs ('actual') as well as in the same image pairs in which one of the channels was rotated 180 degrees to randomize the position of TOM1L1 structures relative to clathrin structures ('rando'). The number of CLSs and cells analyzed are as follows: eGFP-TOM1L1 expression, basal (31 cells, 6574 CLSs), EGF stimulated (37 cells, 8064 CLSs). * ${ }^{*}$ p $<0.05$. 

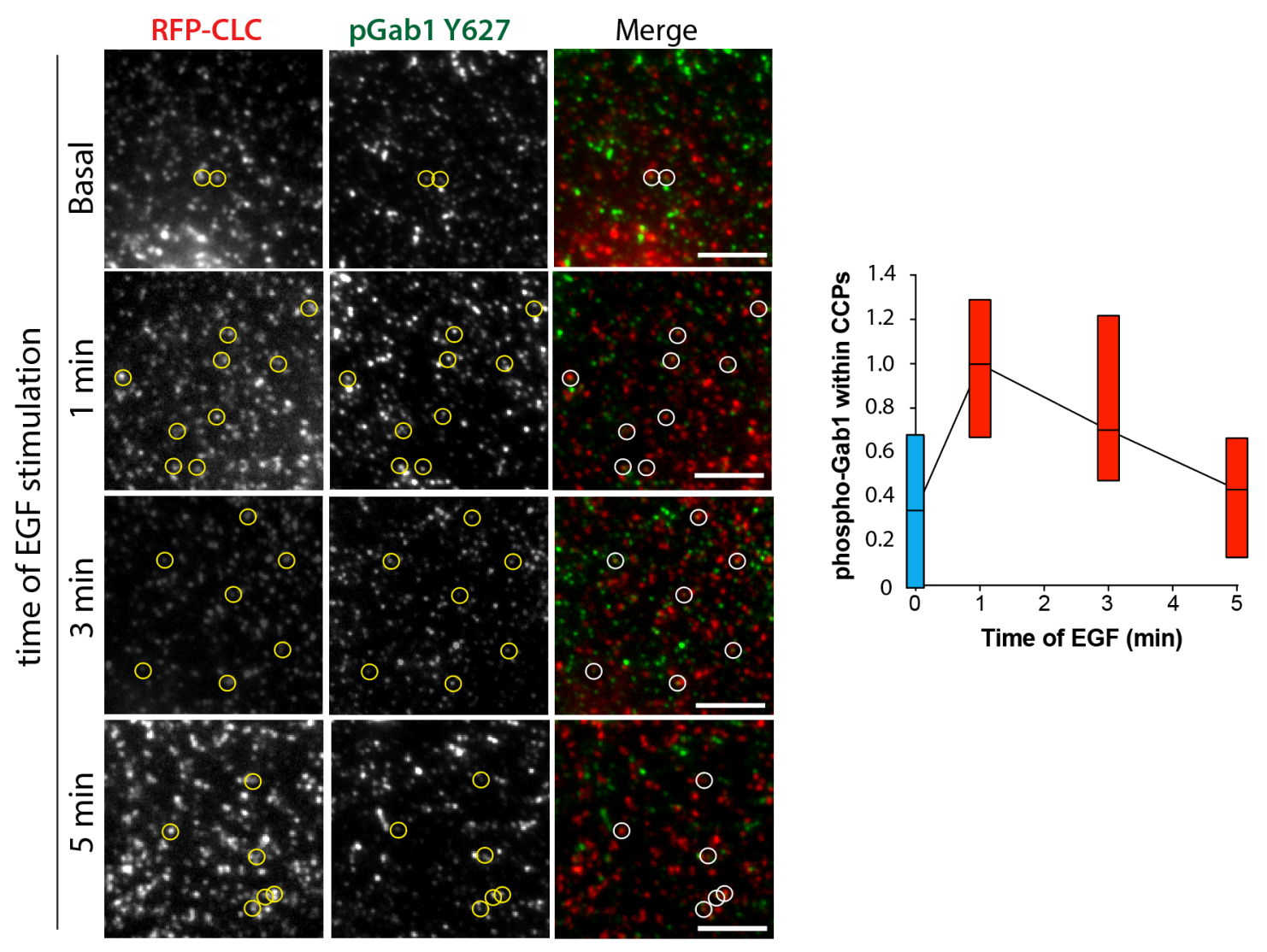

\section{Figure 5.4 Phosphorylated Gab1 is enriched within CCPs upon EGF stimulation.}

RPE cells stably expressing RFP-tagged clathrin light chain (RFP-CLCa) were stimulated with $20 \mathrm{ng} / \mathrm{mL}$ EGF for the indicated times, fixed, and subjected to immunofluorescent staining to detect phosphorylated Gab1 (pGab1) Y627, followed by imaging by TIRF-M. Shown in (A) are representative fluorescence micrographs showing RFP-LCa and A488 stained phospho-Gab1 Y627 channels separately and as a merged image. Circles depict CCPs that exhibit overlap with phosphorylated Gab1, identified manually. Scale $=5 \mu \mathrm{m}$. (B) Images in (A) were subjected to automated detection and analysis of CCPs. Shown are the values for the cellular median (horizontal line) and $75^{\text {th }}$ and $25^{\text {th }}$ percentile values (boxes) for phospho-Gab1 fluorescence intensities within all detected CCPs within each EGF-stimulated condition. The number of CCP (n) and cells $(\mathrm{k})$ for each condition are basal: $\mathrm{n}=49,838, \mathrm{k}=46 ; 1$ min EGF: $\mathrm{n}=59,185, \mathrm{k}=45 ; 3$ min EGF: $n=69,413, k=46 ; 5$ min EGF: $n=57,003, k=46 ; 10 \min E G F: n=54,341, k=46$; 15 min EGF: $\mathrm{n}=49,245, \mathrm{k}=46$. 

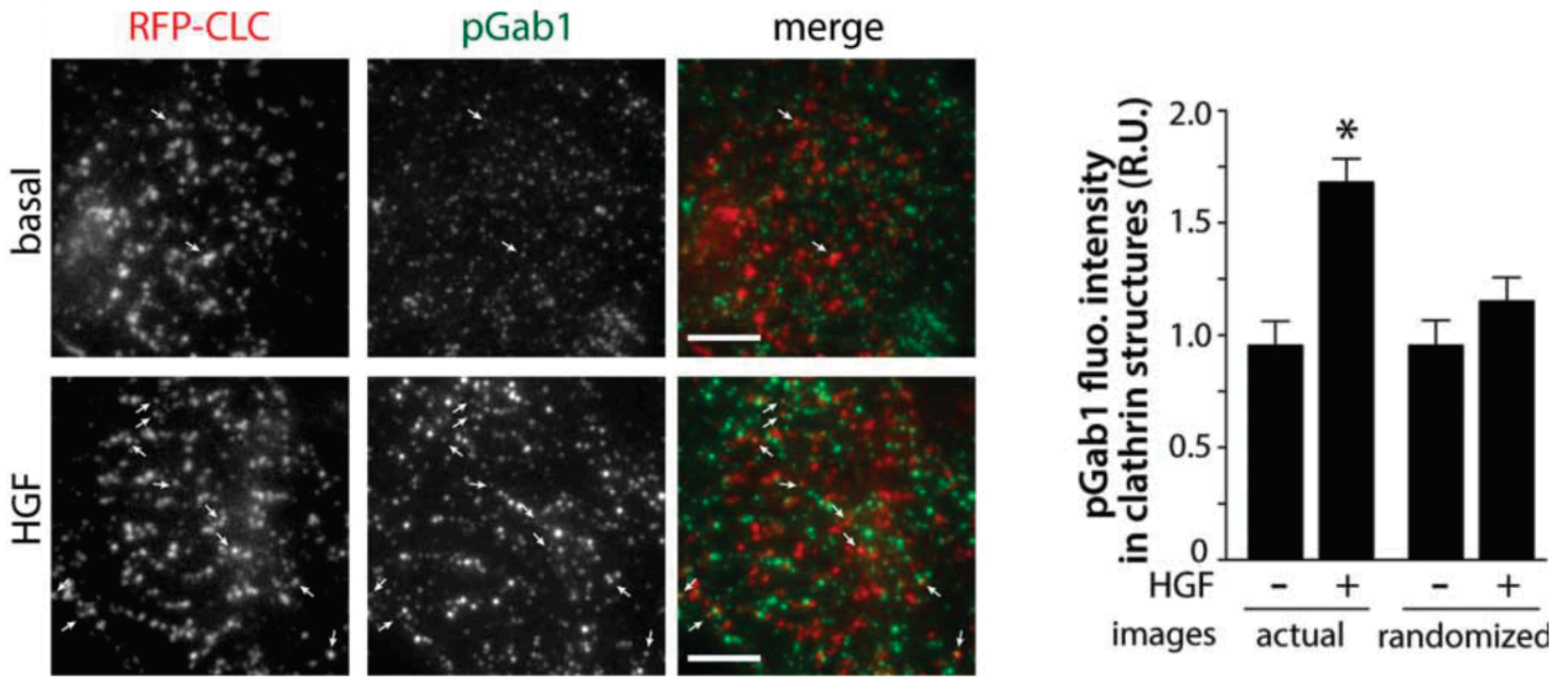

Figure 5.5 HGF stimulation leads to an enrichment of pGab1 within CCPs.

RPE cells stably expressing Tag-RFP-T fused to clathrin light chain (RFP-CLCa) were stimulated with $50 \mathrm{ng} / \mathrm{mL} \mathrm{HGF}$ and immunofluorescently stained for phospho-Gab1 using an antibody that recognizes phospho-Y627. Shown on the left panel are representative micrographs obtained by TIRF-M (scale $5 \mu \mathrm{m}$, arrowheads indicate pGab1-positive CCPs, selected manually). Images obtained by TIRF-M were subjected to automated detection of clathrin structures followed by quantification of pGab1 and RFP-CLC in each detected object. Shown on the right is the mean pGab1 fluorescence intensity detected within clathrin structures in the presence and absence of HGF compared to randomized control ( $>35$ cells per condition from 3 independent experiments). 

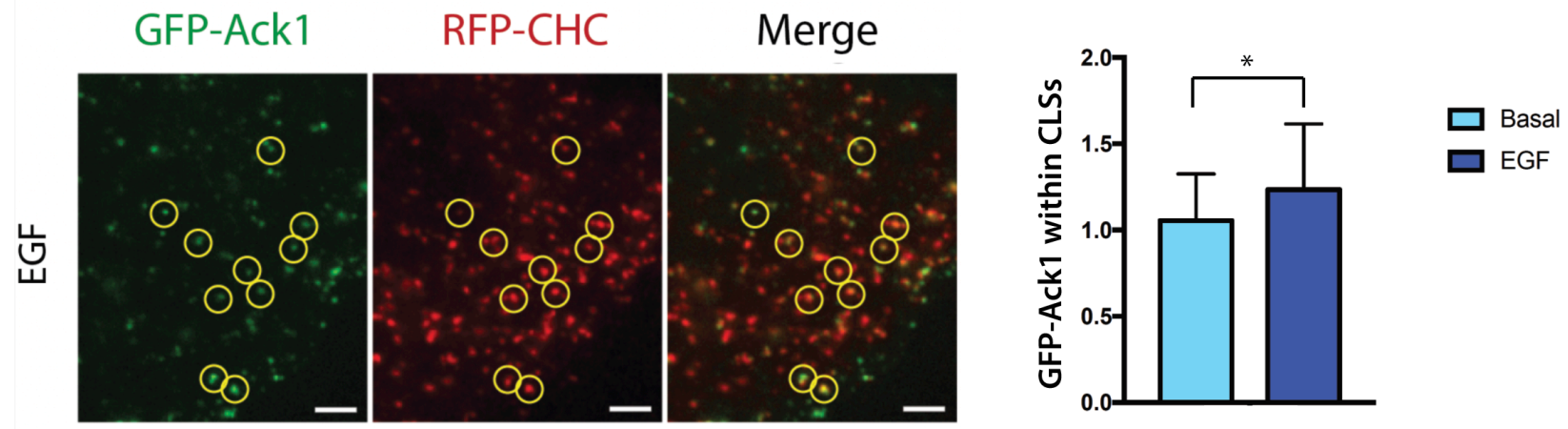

Figure 5.6 Ack1 is enriched within a subset of plasma membrane clathrin structures

RPE cells stably expressing RFP fused to clathrin light chain (Tag-RFP-T-CLC) were transfected with a plasmid encoding eGFP fused to Ack1 (GFP-Ack1). Cells were stimulated with $20 \mathrm{ng} / \mathrm{mL}$ EGF for 5 min or left unstimulated (basal), then fixed and imaged using TIRF-M. Shown (left panel) are representative micrographs depicting Ack1-positive clathrin structures (circles), identified manually. Scale $=5 \mu \mathrm{m}$. Micrographs obtained by TIRF-M were subjected to automated detection and analysis of clathrin structures, allowing quantification of eGFPTOM1L1 and RFP-CLC in each detected object. 


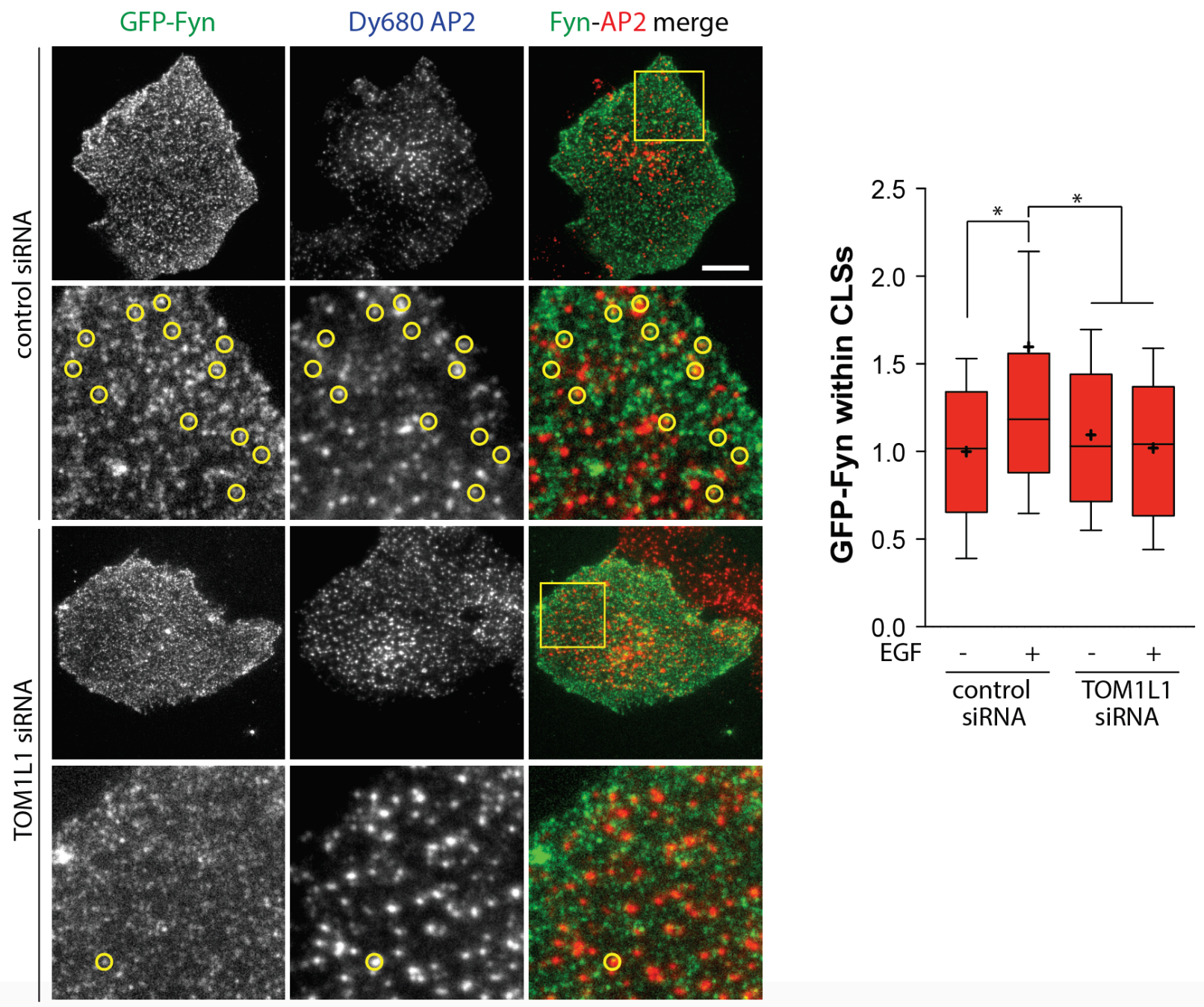

Figure 5.7 TOM1L1 is required for Fyn recruitment to clathrin structures.

RPE cells were transfected with siRNA targeting TOM1L1 or nontargeting siRNA (control), followed by transfection of a plasmid encoding eGFP-tagged Fyn (eGFP-Fyn). Cells were then stimulated with $5 \mathrm{ng} / \mathrm{mL}$ EGF for $5 \mathrm{~min}$, fixed, subjected to antibody staining to fluorescently label AP2, and imaged using TIRF-M. Shown (left panel) are representative micrographs, scale = $5 \mu \mathrm{m}$. Micrographs obtained by TIRF-M were subjected to automated detection and analysis of clathrin structures, allowing quantification of eGFP-Fyn and RFP-CLC in each detected object. Shown (right panel) are the measurements of eGFP-Fyn fluorescence intensity within CLSs, depicted as median (bar) 25th/75th percentiles (boxes) and whiskers (full range). The number of CLSs and cells analyzed, respectively, for each condition are as follows: control siRNA, basal 
(54 cells, 6923 CLSs), EGF-stimulated (39 cells, 5414 CLSs); eGFP-TOM1L1 expression, basal (58 cells, 6640 CLSs), EGF-stimulated (590 cells, 7702 CLSs). 
A GFP-Fyn
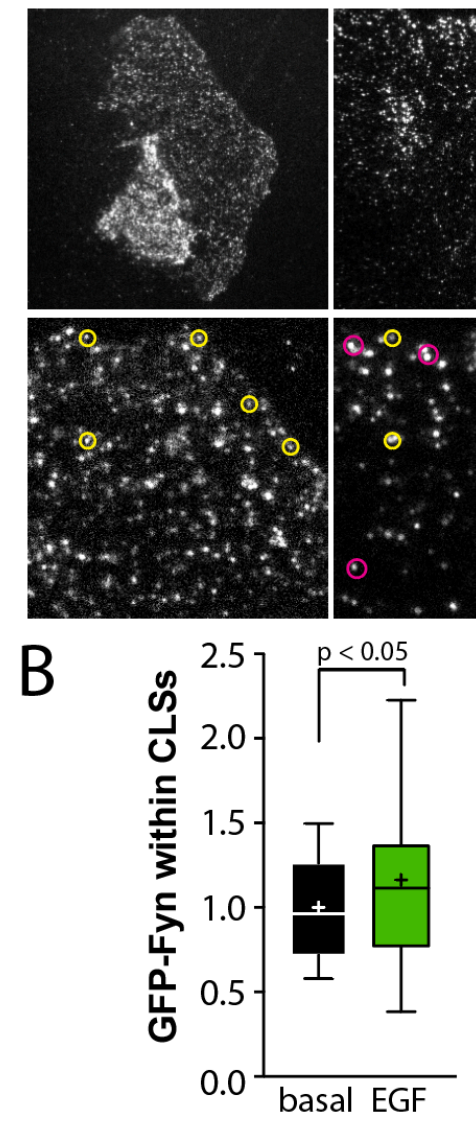

Dy680 AP2
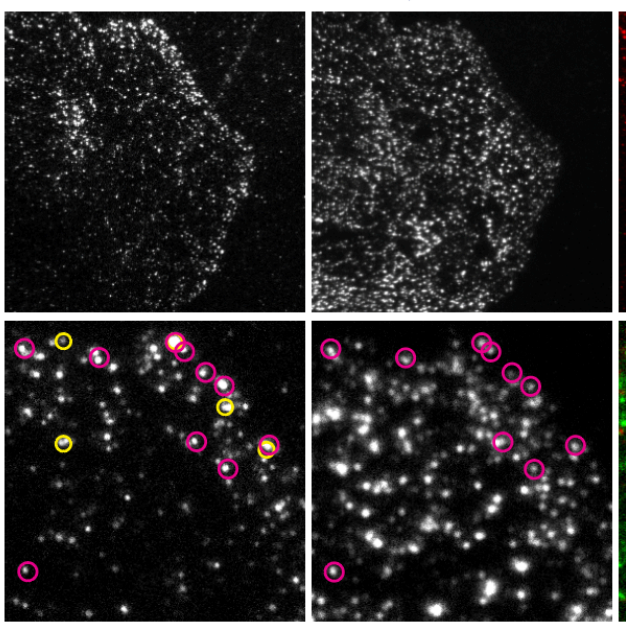

Fyn-EGF merge
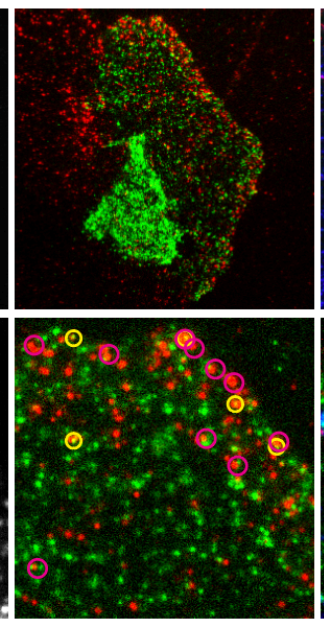

C

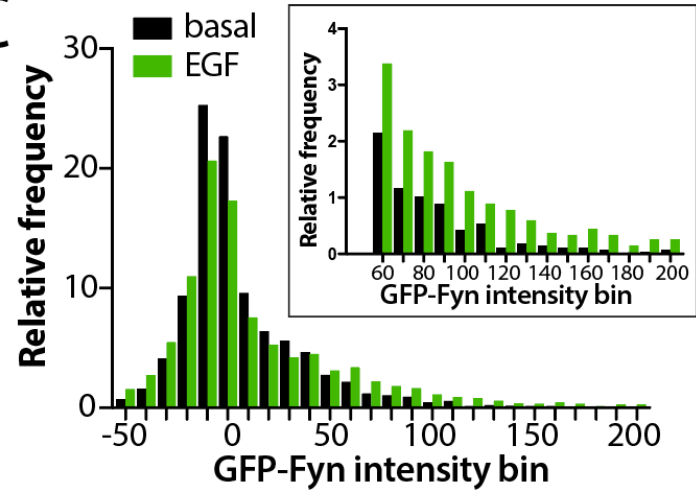

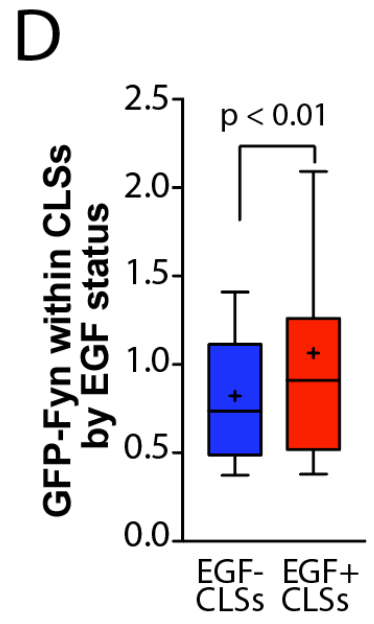

E CLS type
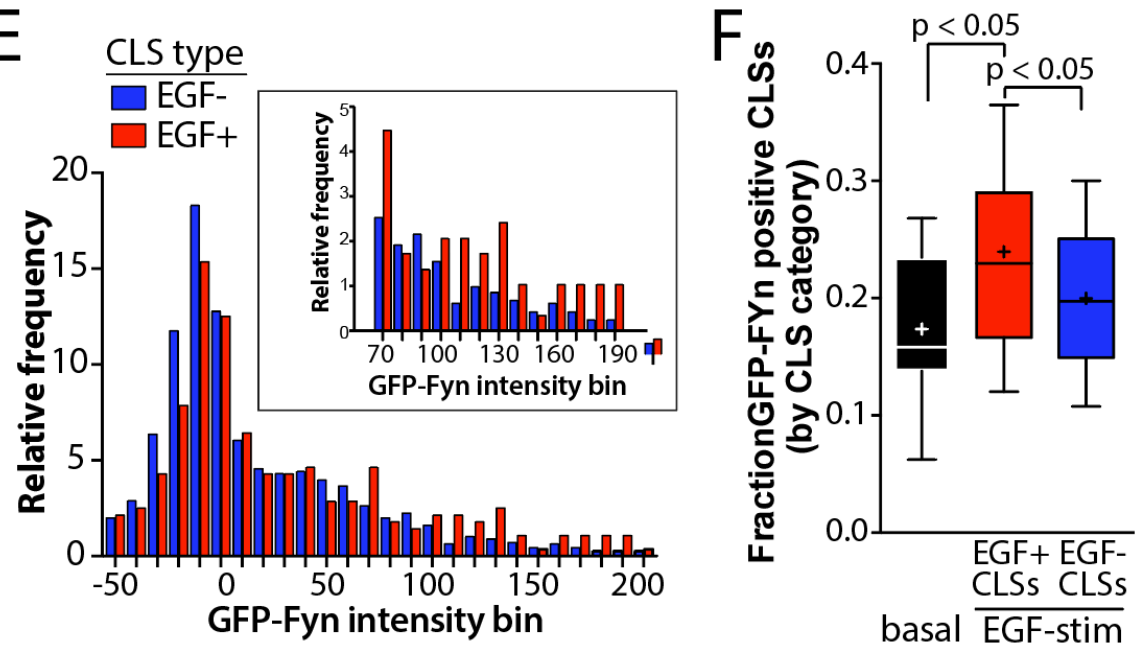

Figure 5.8 Fyn is recruited to a subset of EGF-positive clathrin structures at the plasma membrane.

RPE cells were transfected with a plasmid encoding eGFP-Fyn, stimulated with $20 \mathrm{ng} / \mathrm{mL}$ 
rhodamine-conjugated EGF (Rho-EGF) for $5 \mathrm{~min}$, then subjected to immunofluorescence staining to detect AP2 and finally imaging by TIRF-M. Shown in (A) are representative micrographs, scale $=10 \mu \mathrm{m}$ (top panel) or $5 \mu \mathrm{m}$ (bottom panel). Micrographs obtained by TIRF$M$ were subjected to automated detection and analysis of CLSs, allowing quantification of eGFPFyn, AP2 or Rho-EGF in each detected object. (B) The ensemble average of the levels of eGFPFyn fluorescence within CLSs (detected via AP2), depicted as median (bar) 25th/75th percentiles (boxes) and whiskers (full range). (C) The frequency distribution of eGFP-Fyn within CLSs (detected via AP2) from a representative experiment. (D-F) CLSs (detected via AP2) were subjected to sorting by EGF status (as described in Materials and Methods). Shown in (D) is the ensemble average of eGFP-Fyn within CLSs by EGF status, depicted as median (bar) 25th/75th percentiles (boxes) and whiskers (full range). Shown in (F) is the frequency distribution of eGFP-Fyn within CLSs (detected via AP2) by EGF status from a representative experiment. (F) CLSs (detected via AP2) were subjected to sorting by EGF and eGFP-Fyn status (as described in Materials and Methods). Shown are the mean fraction of Fyn+ CLSs (detected via AP2) in each of the EGF+ and EGF- cohorts, compared to that in basal cells (no EGF). The number of CLSs and cells analyzed, respectively, were 10474 and 44 (from 3 independent experiments). 

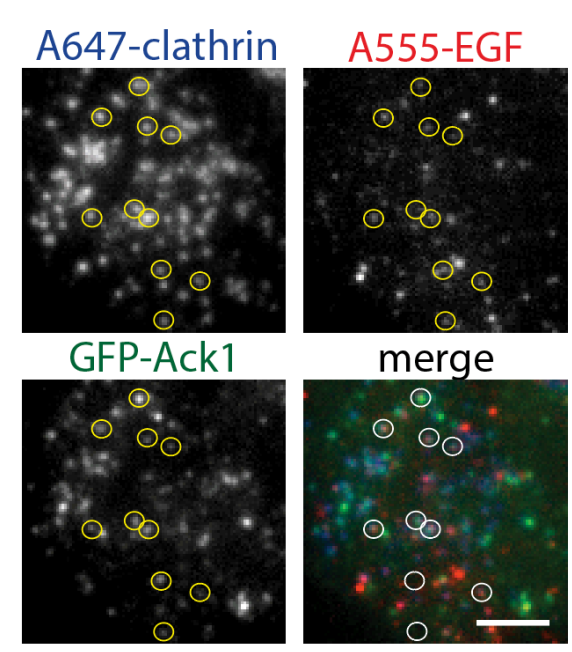
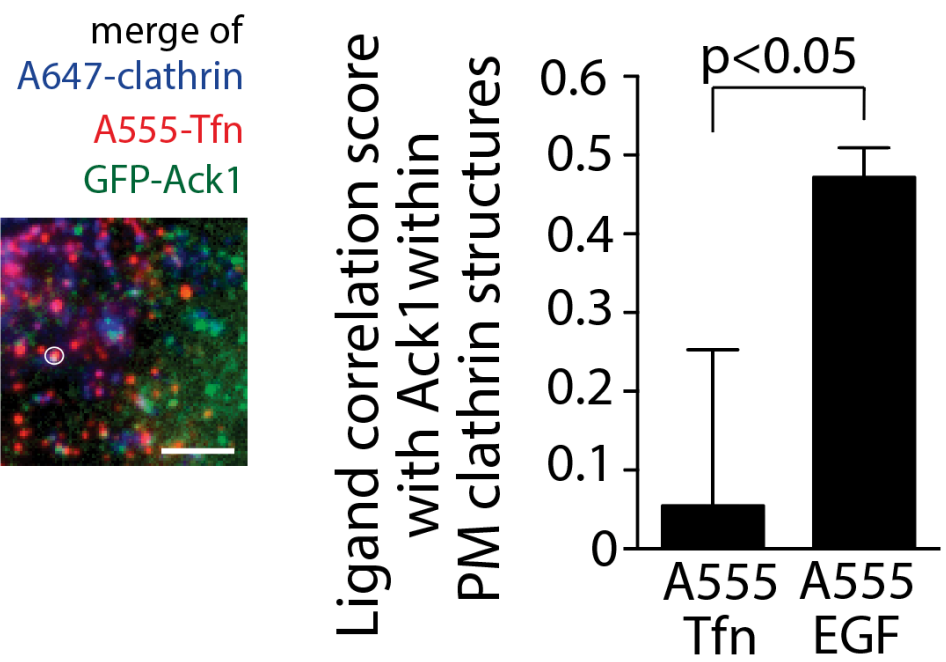

Figure 5.9 Ack1 is detected within EGFR-positive but not TfR-positive PM clathrin structures.

RPE cells were transfected with GFP-Ack1, treated with Alexa555-conj.EGF or Tfn for 3 min (to label EGFR and TfR, respectively), followed by fixation \& labelling with anti-clathrin antibodies (conjugated to A647). Cells were imaged by 3-channel TIRF using a Zeiss Elyra system (at the Hospital for Sick Children). Shown are representative micrographs, showing manual delineation of PM clathrin structures positive for all three markers (EGF/Tfn, Ack1, clathrin) in each cell. EGFR localization with Ack1 in PM clathrin structures is evident, while $\mathrm{TfR} / \mathrm{Ack}$ 1/clathrin overlap is poor. Images were subjected to automated detection of clathrin structures (as per Garay et al. 2015) followed by calculation of a correlation score between Ack1-EGF or Ack1-Tfn fluorescence within each structure. Mean Ack1-ligand correlation scores $\pm \mathrm{SE}$ are also shown. 
A
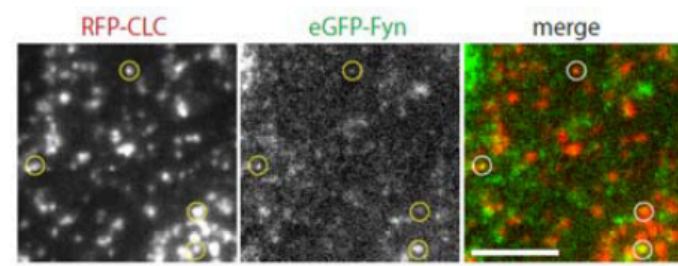

B

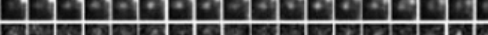

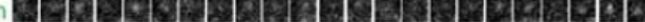

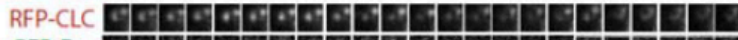

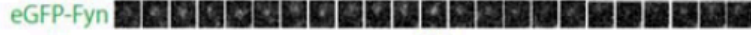

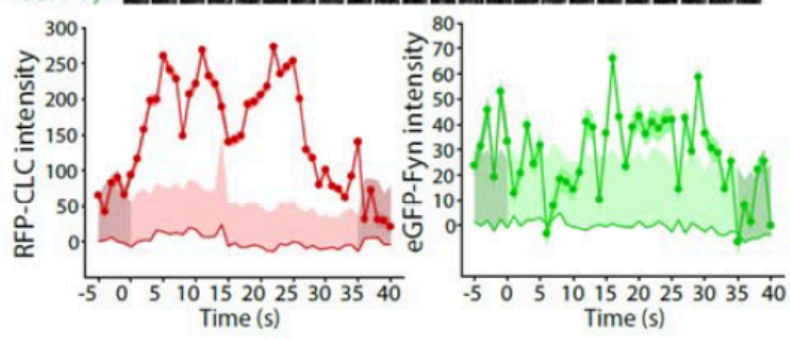

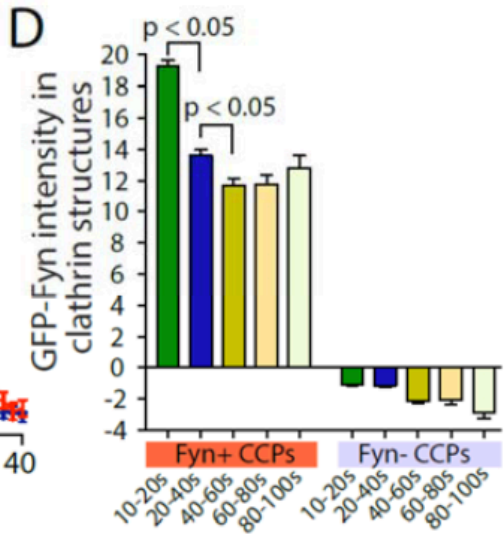

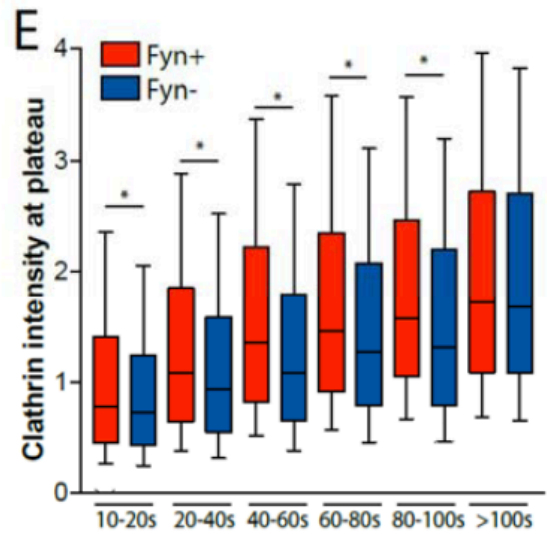

$\mathrm{F}$

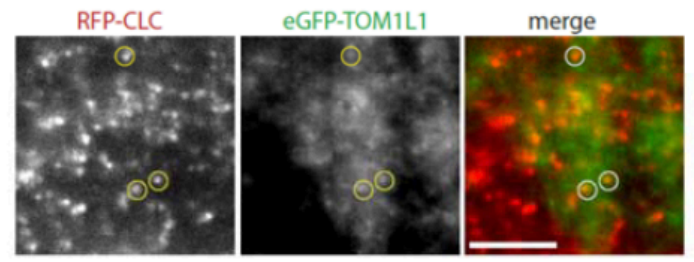

\section{G}

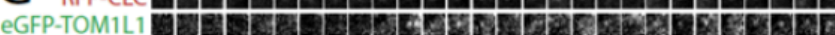

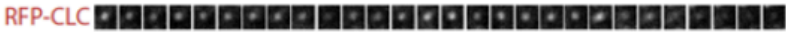

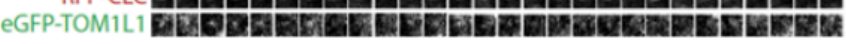
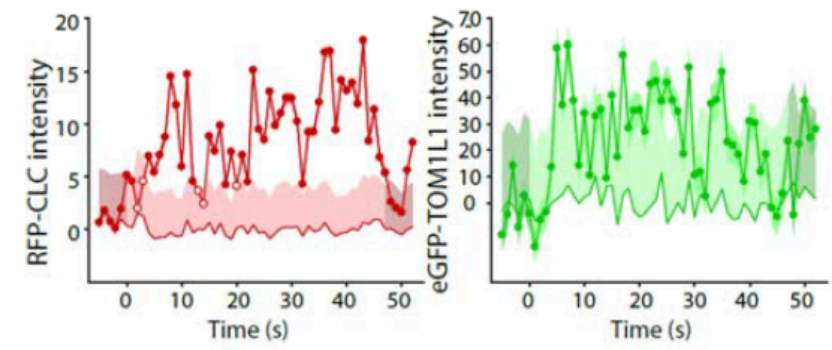
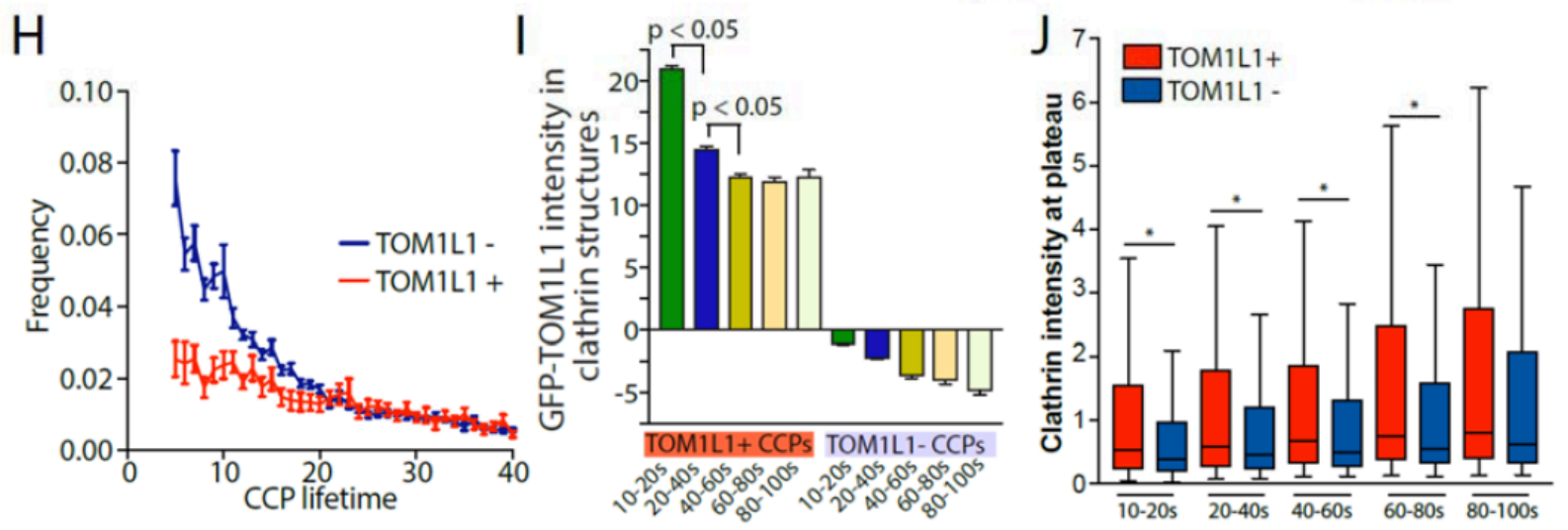
Figure 5.10 Fyn- or TOM1L1-positive CCPs have unique lifetimes and size.

RPE cells stably expressing RFP fused to clathrin light chain (Tag-RFP-T-CLC) were transfected with either eGFP-Fyn (A-E) or eGFP-TOM1L1 (F-J). (A, F) Representative single image frames taken from a sample time lapse. Time-lapse image series were subjected to automated detection, tracking and analysis of CCPs, which allows sorting of CCPs by Fyn status, as described in Materials and Methods. (B, G) Representative Fyn+ (B) or TOM1L1 (G) CCPs showing fluorescence images corresponding to clathrin and Fyn/TOM1L1 centered at the detected object (top panels), as well as quantification of eGFP-Fyn (B) or eGFP-TOM1L1 (G) and RFP-CLC within the object (bottom panels). Additional representative CCPs of each type are available in Figure A4. (C, H) Frequency distribution of CCP lifetimes by Fyn (C) or TOM1L1 (H) status. (D, I) Mean intensity of eGFP-Fyn (D) or eGFP-TOM1L1 (I) within each type of CCP (sorted by automated analysis first by Fyn/TOM1L1 status, then by CCP lifetime). (E, J), Mean intensity of RFP-CLC within each type of CCP (sorted by automated analysis first by Fyn/TOM1L1 status, then by CCP lifetime). *, $\mathrm{p}<0.05$. The mean intensity of eGFP-Fyn, eGFP-TOM1L1, or RFP-CLC within CCPs is determined by the mean plateau intensity of each fluorescently-labelled protein in each detected object, as described in Materials and Methods. The number of CCPs and cells analyzed, respectively, for each condition are as follows eGFPFyn expressing cells (18383 CCPs, 17 cells) and eGFP-TOM1L1 expressing cells (28234 CCPs and 19 cells). 

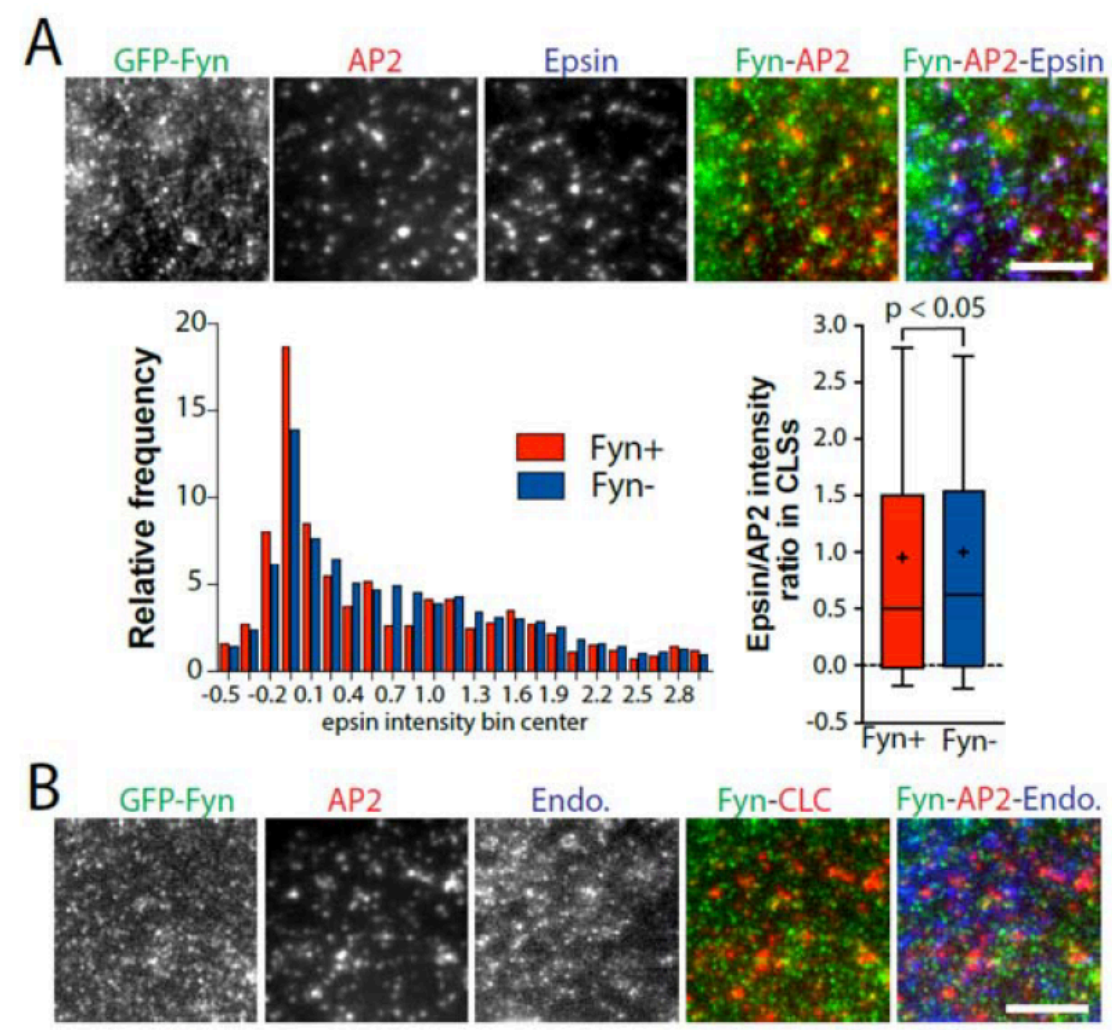

Endo.

Fyn-CLC Fyn-AP2-Endo.
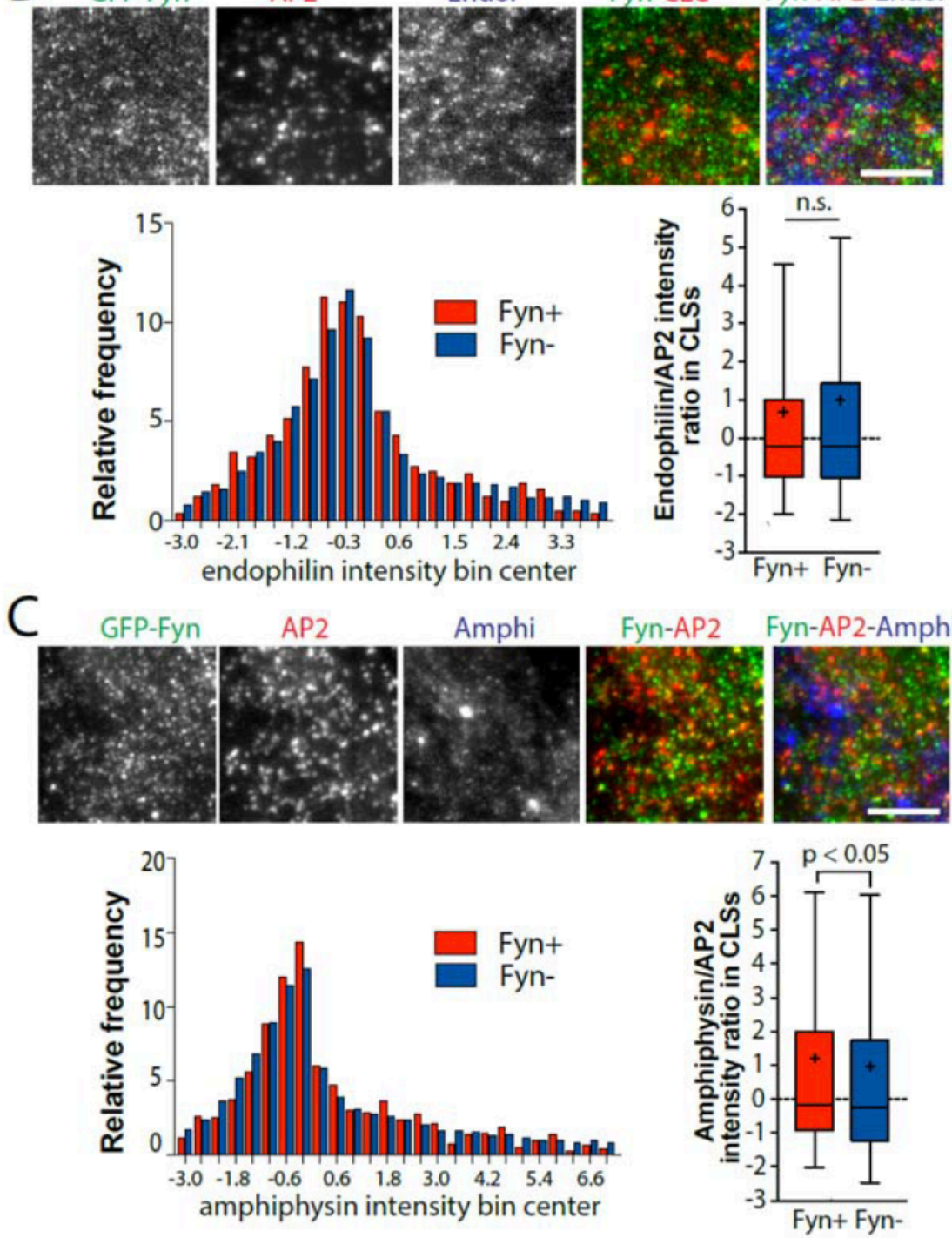
Figure 5.11 GFP-Fyn positive clathrin structures contain a unique profile of endocytic accessory proteins.

RPE cells were transfected with eGFP-Fyn, stimulated with $20 \mathrm{ng} / \mathrm{mL}$ EGF for $5 \mathrm{~min}$, then fixed and subjected to immunofluorescence staining of AP2 and either Epsin (A), Endophilin (B), or Amphiphysin (C). Shown for each (top panels) are representative micrographs depicting Fynpositive clathrin structures. scale $=5 \mu \mathrm{m}$. Micrographs obtained by TIRF-M were subjected to automated detection and analysis of clathrin structures, allowing quantification of each protein within each CLS (detected via AP2), as well as sorting of CLS by Fyn status (as described in Materials and Methods). Shown (bottom left panels) is the ensemble average of the ration of epsin (A), endophilin (B) or amphiphysin (C) levels to that of AP2 within Fyn+ or Fyn- CLSs, depicted as median (bar) 25th/75th percentiles (boxes) and whiskers (full range). Also shown for each (bottom right panels) is the frequency distribution of the ratio of epsin (A), endophilin (B) or amphiphysin (C) to AP2 within CLSs from a representative experiment. The number of CLSs and cells analyzed, respectively, for each condition are as follows: endophilin-labelled cells (20266 CLSs, 33 cells), epsin-labelled cells (21110 CLSs, 40 cells), and amphiphysin-labelled cells (24545 CLSs, 42 cells). 


\subsection{Additional Contributions}

Foreign materials in the body are engulfed and degraded by professional phagocytic cells, and used for the establishment of the body's immunological response and in tissue homeostasis (Prashar et al., 2013). These phagocytic cells use phagocytic receptors and F-actin dependent formation of pseudopodia to bind target particles and engulf them into a phagocytic cup that eventually internalizes the material and targets it for degradation in the lysosome (Viera et al., 2002). Through studying filamentous Legionella pneumophila (Lp), we now know that the morphology of the pathogen affects the outcome of phagocytosis, and the internalization of filamentous bacteria deviates from the canonical phagocytic pathway that has been delineated for spherical phagocytic targets. Internalization of filamentous $L p$ occurs through a long-lasting tubular phagocytic cup that occurs in an orientation-dependent manner, and internalized filaments undergo fragmentation to produce infectious progeny in the cell (Prashar et al., 2013).

$L p$ is a human pathogenic bacterium that causes Legionnaires' disease, an atypical form of pneumonia treated with antibiotics. $L p$ has been shown to infect lung epithelial cells (LECs) by first binding and engaging the host cell's $\beta 1$ integrin and E-Cadherin receptors, followed by a still unknown signaling event that leads to the actin-dependent formation of filopodial and lamellar membrane hooks and wraps across the length of the Lp, respectively (Prashar et al., 2013). The membrane wraps engulf the filamentous bacteria over time and leads to the internalization of the bacterial filament into a pre-vacuolar compartment of the LEC called a Legionella containing vacuole (LCV). It has previously been shown that SFKs, class IA PI3K, and Rho family GTPases are among host target proteins exploited by the bacteria in order to achieve actin-dependent uptake in non-phagocytic cells (Heasman \& Ridley, 2008). I was able to engage in a collaboration with the lab of Dr. Mauricio Terebiznik at the University of Toronto 
Scarborough to study the signaling pathways activated by $L p$ upon binding to LEC. In Prashar et al., 2018, I contributed to the investigation of the molecular mechanism responsible for actindependent rearrangement needed for the formation and elongation of these membrane wraps and bacterial internalization. Specifically, I explored the contribution of SFK signaling to $L p$ infection in LECs and probed activation of the PI3K pathway by investigating phosphorylation of its downstream target Akt following $L p$ infection. These targets were chosen as they have previously been shown to be critical regulators of actin polymerization downstream of $\beta 1$ integrin and E-Cadherin activation (McLachlan et al., 2007; Pang et al., 2005).

There was a significant increase in activation of Src as probed by an antibody recognizing phosphorylation of Y416, and activation increased as the $L p$ infection period length increased (see figure below). There was also a significant increase in Akt phosphorylation at S473, the robustness of which also coincided and increased with length of infection period. Additional experiments done by other authors of the paper demonstrated that the SFK inhibitor PP2 and the PI3K inhibitor LY294002 both caused a marked reduction in attachment of filamentous $L p$ to host cells, together demonstrating a role for Src and PI3K in the attachment of $L p$ to LECs. 

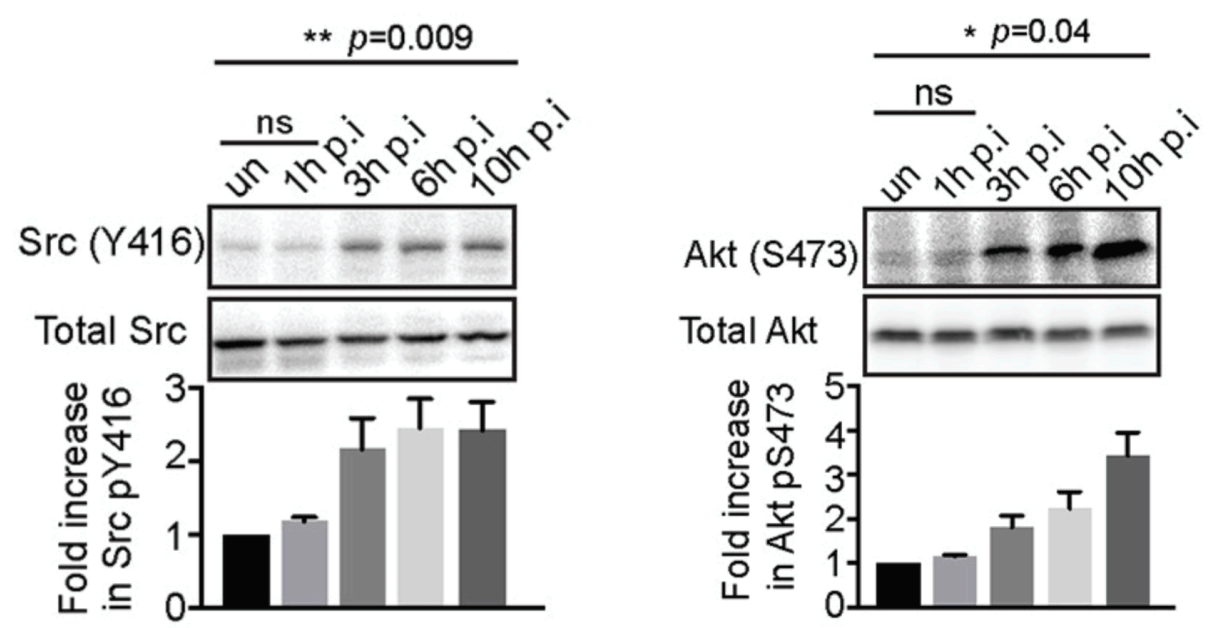

Figure A1 Attachment of filamentous Legionella pneumophila to lung epithelial cells requires Src and PI3K activity.

LECs were infected with Lp for the times indicated above, and whole cell lysates were prepared for immunoblotting. Levels of Src (Y416) and Akt (S473) phosphorylation in cells infected with $\mathrm{Lp}$ for the indicated times were probed using antibodies. Blot shown for Src (Y416) is representative from 9 independent experiments, and blot shown for Akt (S473) is representative from 4 different experiments. Statistical analysis was performed using multiple comparisons oneway ANOVA. 


\section{Chapter 6. Discussion}

It is evident that the plasma membrane is a highly heterogeneous environment-facing organelle, containing a diverse complement of proteins that the cell uses to sense, respond, and adapt to changing environmental conditions. In addition to its heterogeneous complement of proteins found there, the plasma membrane is compartmentalized into a number of distinct microdomains that allow context-specific control of cellular processes such as metabolism, migration, growth, and proliferation through spatial and temporal regulation of RTKs resident herein. Internalization and turnover of integral membrane proteins and RTKs following ligand engagement or changes in the needs of the cell are facilitated primarily by CME, making this process a key regulator of the cell surface proteome. RTKs are recruited to and enriched within various plasma membrane microdomains under diverse conditions, and facilitate the activation of many intracellular signal transduction pathways from here, including PI3K/Akt, Ras-MAPK, PLC, JAK-STAT, and Rho-family GTPases (Lemmon \& Schlessinger, 2010).

To date, membrane rafts, caveolae, tetraspanin-enriched domains, and actin-dependent membrane protrusions have all been characterized as bona-fide microdomains within the plasma membrane, with evidence that each can regulate the signaling of certain receptors under specific contexts (Delos Santos, Garay, \& Antonescu, 2015). In this thesis, I define a role for plasma membrane clathrin nanodomains in the control of RTK signaling and propose that the preferential recruitment of a specific subset of endocytic accessory proteins and signaling effectors to a subset of CCPs imparts upon these structures the properties of a signaling nanodomain. Specifically, I found that i) clathrin is required for activation of the PI3K/Akt pathway downstream of both EGFR and Met receptor, and that perturbation of clathrin does not affect activation of EGFR itself upon ligand binding. Additionally, I explored the contribution of 
a complement of endocytic accessory proteins and non-receptor tyrosine kinases to EGFR signaling and found that ii) Fyn, TOM1L1 and Ack1 are required for phosphorylation of key PI3K/Akt pathway intermediate Gab1, and downstream phosphorylation of Akt. Lastly, using advanced imaging techniques including TIRF-M and SIM, I have iii) characterized CCPs as heterogenous structures containing unique combinations of receptors and endocytic accessory proteins, such that a specific subset of CCPs exhibit distinct dynamic properties and harbor key signaling regulators. Collectively, these findings reveal a mechanism by which clathrin controls RTK signaling at the plasma membrane through formation of a clathrin-labeled signaling nanodomain, to be discussed in further detail below.

\subsection{Regulation of PI3K/Akt signaling by clathrin}

As described in Section 1.1.3, one of the pathways activated downstream of EGFR is the PI3K/Akt signaling axis, which controls cell proliferation, metabolism, and inhibition of apoptosis. Peak phosphorylation of Akt has been reported to occur 1-3 mins following stimulation of EGFR with its ligand, EGF (Borisov et al., 2009; Kiyatkin et al., 2006; Sorkin \& Goh, 2009), which coincides with recruitment of EGFR to CCPs and receptor activation. Given that EGFR residence within CCPs occurs concomitantly to receptor activation, I first examined the requirement for clathrin in controlling signaling downstream of EGFR in RPE cells. Perturbation of clathrin by siRNA (Figure 3.1) or pharmacological inhibition using Pitstop2 (Figure 3.4) significantly reduced EGF-stimulated Akt phosphorylation, however, perturbation of Dynamin 2 by siRNA (Figure 3.1) did not significantly alter EGF-stimulated Akt phosphorylation. These findings indicate that clathrin plays a direct role in regulating EGFR signaling at the plasma membrane, and consistently that perturbation of Dynamin 2, which allows the formation of CCPs at the plasma membrane but not their eventual scission to create 
intracellular CCVs, does not affect signaling leading to Akt activation. In addition to these findings, perturbation of clathrin using siRNA or pharmacological inhibition with Pitstop2 is also required for EGF-stimulated Gab1 phosphorylation in RPE cells (Garay et al., 2015). It is possible that perturbation of clathrin through siRNA gene silencing may have off-target cellular effects, which is why two distinct sequences targeting different sites on $\mathrm{CHC}$ were created to mitigate this. Indeed, the two sequences had a similar effect on EGF-stimulated Akt phosphorylation (Figure 3.1).

Moreover, it is also possible that perturbation of clathrin and inhibition of Akt phosphorylation downstream of EGFR is a result of alteration of the receptor itself upon clathrin perturbation. This possibility was addressed using $\mathrm{CHC}$ silencing as well as treatment with Pitstop 2 followed by measuring both activation of EGFR and its total levels on the cell surface. Perturbation of clathrin using siRNA or Pitstop 2 was without effect on i) EGF-stimulated EGFR phosphorylation as measured by immunofluorescence microscopy using an antibody that recognizes phospho-EGFR Y1068 (Figure 3.2A), ii) EGF-stimulated EGFR phosphorylation measured by immunoblotting whole cell lysates with antibodies that recognize various known phosphorylation sites on EGFR's C-terminus (Figure 3.2B), and iii) cell-surface EGFR levels, measured by an antibody that selectively recognizes the EGFR ectodomain in intact cells (Figure 3.3). Taken together, the results in Chapter 3 of this thesis as well as the additional experiments published in Garay et al. (2015) provide strong evidence that clathrin is required for EGF-stimulated phosphorylation of Akt in a manner independent of or subsequent to receptor activation.

CME is responsible for the down-regulation of numerous receptors from the plasma membrane, which include EGFR as well as many others such as TfR, Insulin (Martins et al., 
2011), RET (Crupi et al., 2014), and Met (Hammond et al., 2001; Li et al., 2007) receptors. To determine whether this novel function of clathrin in control of receptor signaling at the plasma membrane extends to other RTKs, I next chose to examine the HGF receptor, Met. Upon binding its ligand HGF, the Met receptor homodimerizes and phosphorylates tyrosine residues within its C-terminus which recruit various signaling intermediates, just as with EGFR binding EGF (Organ \& Tsao, 2011). Unlike EGFR however, Met receptor is able to directly bind to Gab1 without the aid of Grb2 (Weidner et al., 1996). HGF stimulation in RPE cells results in robust phosphorylation of Gab1 at Y627, comparable to that of EGF stimulation, however does not elicit phosphorylation of EGFR (Figure 3.4A), demonstrating that the cells respond effectively and specifically to HGF. To examine the role of clathrin in Met signaling, RPE cells were treated with Pitstop2 to inhibit clathrin mediated endocytosis and stimulated with either EGF or HGF. Similarly to the effect of clathrin perturbation in Figure 3.1, treatment with Pitstop2 significantly reduced HGF-stimulated Akt phosphorylation as well as EGF-stimulated Akt phosphorylation (Figure 3.4). We can therefore infer that there is a similar requirement for clathrin in EGF- and HGF-stimulated Akt phosphorylation, and that the function of clathrin as a signaling regulator extends to receptors beyond EGFR.

Notably, this function of clathrin is not required by all RTKs internalized through CME, as its perturbation using Pitstop2 has no effect on EGF-stimulated Akt phosphorylation in cells expressing ErbB2 and thus signaling from EGFR-ErbB2 heterodimers rather than EGFR-EGFR homodimers (Garay et al., 2015). ErbB2 is a member of the ErbB family of receptors along with EGFR, ErbB3 and ErbB4, however in contrast to its other family members, ErbB2 has no known ligands and only becomes activated through heterodimerization with other ligand-bound receptor family members (Hynes \& MacDonald, 2009). ErbB2 is found to be overexpressed in 20-30\% of 
all human breast cancer cases and 40-60\% of all ductal carcinomas (Espina \& Liotta, 2011). The first approved therapy that targeted ErbB2 positive breast cancers was Trastuzumab (Herceptin), a monoclonal antibody that binds the extracellular domain of ErbB2 and inhibits proliferation (Harari \& Yarden, 2000; Rimawi et al., 2015). Trastuzumab has had some success in treating ErbB2 positive breast cancers, however resistance to it has been reported to occur (Pohlmann et al., 2009). Overall, ErbB2 positive cancers are correlated with poor prognosis (Espina \& Liotta, 2011), and it is therefore important that we study and delineate the signaling events activated downstream of this receptor as an ongoing part of cancer research.

At the cellular level, ErbB2 is 100 -fold more potent in its transforming ability than EGFR when activated, despite the two receptors sharing over $85 \%$ sequence homology and sharing activation of similar signaling pathways downstream of activation (Di Fiore et al., 1987; Hynes \& MacDonald, 2009). One difference in signaling downstream of these receptors however is in their ability to bind SFKs, specifically c-Src. c-Src is able to directly bind the kinase domain of ErbB2 at Y877 independently of its own activation; however, c-Src cannot bind EGFR directly (Marcotte et al., 2009). The SFKs are important signaling proteins downstream of the ErbB receptor family as phosphorylation of Y845, Y891, Y920, and Y1101 on EGFR are dependent on their activity (Biscardi et al., 1999). While c-Src is not able to bind EGFR directly, it is nonetheless essential for EGFR function. Cells expressing kinase deficient c-Src or phospho-null point mutations (Y845F) are unable to undergo EGFR phosphorylation at the aforementioned sites, resulting in decreased overall cell proliferation and tumorigenesis, and an impairment in their ability to synthesize DNA in response to EGF treatment (Tice et al., 2002; Biscardi et al., 1999). 
Two mutations in EGFR, L835R and $\Delta 722-726$, account for approximately $90 \%$ of all EGFR lung cancer mutations and confer increased sensitivity to two cancer drugs that specifically inhibit EGFR kinase activity, gefitinib and erlotinib (Marcotte et al., 2009). Unfortunately, more than $50 \%$ of lung cancer patients with these mutations that respond well to the kinase inhibitors eventually acquire additional somatic mutations in EGFR that render them resistant to the two drugs, highlighting the need for new cellular drug targets for treatment of resistant forms of EGFR-driven cancers (Brewer et al., 2013; Yatabe \& Mitsudomi, 2007). Interestingly, L835R and $\Delta 722-726$ gain the capacity to associate with c-Src directly just as ErbB2 does, and transformation of cells harboring these mutations become sensitive to SFK inhibitors regardless of their sensitivity or resistance to gefitinib and erlotinib (Marcotte et al., 2009). The possibility that clathrin acts as a signaling scaffold, coordinating the recruitment and activity of endocytic accessory and signaling proteins at the plasma membrane following EGF stimulation is therefore a likely explanation for the role of clathrin in EGFR but not ErbB2 signaling. Since ErbB2 can bind such important signaling regulators as c-Src directly, it forgoes the requirement for a signaling scaffold to bring these proteins into proximity of the receptor, and becomes insensitive to clathrin perturbation. Lung cancer mutations in EGFR that allow it to bind c-Src may also forego the requirement for clathrin as a signaling scaffold, as these mutated receptors exhibit 24-fold increased transformation potential compared to wild type EGFR (Marcotte et al., 2009). Not only do these studies highlight the importance of SFKs in ErbB receptor signaling, it also represents an important future direction for SFK research in EGFR driven cancers.

From this, it becomes clear that the future directions of this research include a broader study of ErbB2-positive breast and lung cancers, as understanding the signaling downstream of 
these receptors is necessary to understand how signaling from the ErbB family is dysregulated. Since c-Src binds ErbB2 directly, it would be of interest to obtain siRNA to c-Src directly and determine whether gene silencing of this SFK inhibits the ability of signaling in these ErbB2 driven cell lines to activate Akt downstream. In addition to siRNA, I would complement this experiment with pharmacological inhibition of SFKs using Dasatinib, as I have done in RPE cells expressing EGFR and not ErbB2. Since these cells are not sensitive to perturbation of clathrin and forego the requirement of a signaling nanodomain, the direct recruitment of c-Src to ErbB2 could be mediating signaling downstream of this receptor. If this is true, then perturbation of c-Src directly by gene silencing or Dasatinib treatment would inhibit signaling downstream.

In summary, we have revealed a novel function of clathrin in regulating EGFR and Met signaling from the plasma membrane leading to Akt phosphorylation, in addition to its wellknown function in receptor-mediated endocytosis. This novel function of clathrin does not extend to all RTKs however, as cells expressing ErbB2 and therefore signaling via EGFR-ErbB2 heterodimers forego the requirement of clathrin in EGF-stimulated Akt phosphorylation. Context-specific signaling and spatial organization of RTKs may therefore be regulated by clathrin nanodomains at the plasma membrane, extending our knowledge of known RTK signaling effectors.

\subsection{Regulation of PI3K/Akt signaling by Fyn and TOM1L1}

In order to study the role of SFKs on clathrin dependent EGF-stimulated Akt phosphorylation, I used the SFK specific inhibitor Dasatinib, a more selective inhibitor of SFK signaling with fewer off target effects than first generation SFK inhibitors PP1 and PP2 that have conventionally been used in studies of SFK signaling (Fu et al., 2008; Kassenbrock et al., 2002; Sorkina et al., 2002). Using Dasatinib first at high concentrations $(1 \mu \mathrm{M})$, treatment of RPE cells 
with the inhibitor resulted in a significant reduction of signal detected by an antibody that recognizes the phosphorylated form of a conserved motif on Src-family kinases (Y416 on c-Src)

(Figure 4.1). Treatment with $1 \mu \mathrm{M}$ Dasatinib also inhibited EGF-stimulated Akt and Gab1 phosphorylation, as expected (Figure 4.1), however this effect did not reflect a change in EGFR activation or phosphorylation at key residue Y1068 (Figure 4.1).

Interestingly, although the intensity of signal detected in phospho-EGFR Y1068 was unaffected by treatment with high concentrations of Dasatinib, the detection of higher molecular weight species of EGFR was reduced by $1 \mu \mathrm{M}$ Dasatinib treatment. This can be seen in Figure 4.1, as the band detected in control lanes of the immunoblot appear smeared above the main intense band recognizing phospho-Y1068. EGFR is dynamically altered by post-translational modifications including phosphorylation of its $\mathrm{C}$-terminus following receptor activation and ubiquitination by the $\mathrm{E} 3$ ubiquitin ligase $\mathrm{Cbl}$ influencing degradation (Carpentersllil, 1984; Kaszuba et al., 2015; Soubeyran et al., 2002; Tong et al., 2014). Each of these modifications therefore may alter the molecular weight of EGFR.

On why treatment with $1 \mu \mathrm{M}$ Dasatinib reduced the detection of these higher molecular weight EGFR species, there are several possible explanations. Since SFKs are required for the direct phosphorylation of EGFR at multiple residues (Bromann et al., 2004; Lu et al., 2009), the loss of detectable higher molecular weight EGFR species could reflect a loss of SFK activity in the cells and a decrease in phosphorylation at sites other than Y1068. In addition, c-Src is known to influence phosphorylation of Y1045 on EGFR (Ravid et al., 2002), which is also the site at which $\mathrm{Cbl}$ binds EGFR directly, a critical step that subsequently allows $\mathrm{Cbl}$ to mediate ubiquitination leading to lysosomal sorting and degradation (de Melker et al., 2001; Grøvdal et al., 2004; Soubeyran et al., 2002). A loss of higher molecular weight EGFR species upon 
treatment with Dasatinib may therefore be a reflection of a loss of ubiquitination of the receptor as well. Taken together, this result demonstrates that although ligand-dependent activation of the receptor leading to auto-phosphorylation at Y1068 (required for PI3K/Akt pathway activation) is unaffected by treatment with $1 \mu \mathrm{M}$ Dasatinib, there may be off target effects at this high concentration or changes in receptor trafficking. This in turn suggests that this concentration of Dasatinib is potentially altering other receptor modifications, such as other phosphorylation sites or receptor ubiquitination. This highlights the need for more studies using lower, more selective concentrations of this inhibitor, providing a major topic of investigation for this thesis.

I next aimed to inhibit SFKs in RPE cells using a lower, more specific dose of Dasatinib $(50 \mathrm{nM})$ as previously reported (Nam et al., 2005). Following treatment with $50 \mathrm{nM}$ Dasatinib, phosphorylated SFK signal was again virtually completely lost, however Akt phosphorylation was largely unaffected (Figure 4.2). This is the first time that a disconnect between the EGFstimulated activation of Akt and the functional requirement for SFKs has been shown, as most previous studies using SFK inhibitors report a loss of Akt activation following Src inhibition (Kassenbrock et al., 2002; Ueda et al., 2009). However, these previous studies didn't investigate low dose treatment of SFK inhibitors, therefore the effects they observed with SFK inhibitor may have potentially been driven by off-target effects of the drug including inhibition of EGFR phosphorylation or membrane traffic dynamics (Nautiyal et al., 2009; Sorkina et al., 2002). On why $50 \mathrm{nM}$ Dasatinib treatment results in inhibition of Src activation but not Akt (Figure 4.2), this finding may indicate the role of a Dasatinib-insensitive SFK or the requirement of a different cytosolic kinase in EGF-stimulated Akt phosphorylation.

To explore the possibility that a Dasatinib-insensitive SFK may play a role on EGFstimulated Akt phosphorylation, I next looked to determine whether Dasatinib had an effect on 
SFKs other than the species being detected by the phospho-Src Y416 antibody in Figure 4.1 and Figure 4.2. Despite claims from its manufacturer that the antibody is able to recognize phosphorylated forms of all SFKs, immunoblotting with phospho-Src Y416 often resulted in only one visible band that corresponds to the molecular weight of c-Src, which is distinct from that of other SFKs such as Fyn (as discussed in Chapter 4). This suggests that while anti-SFK antibodies may be capable of recognition of the phosphorylated forms of many or all SFKs, each antibody may preferentially recognize a specific SFK. The antibody also may be detecting higher levels of certain SFKs dependent on relative expression level of each SFK in RPE cells. As quantified in Figure 4.1, phosphorylation of the SFK detected by the phospho-Src Y416 antibody following $1 \mu \mathrm{M}$ Dasatinib treatment in three separate experiments resulted in only a $60 \%$ decrease in overall phospho-SFK signal, and phosphorylation of the SFK(s) detected was not enhanced in response to EGF as has previously been reported in some contexts (Wilde et al., 1999). The remaining signal detected on these immunoblots may therefore be due to the presence of a more Dasatinib-insensitive SFK, and not due to incomplete inhibition of all SFKs.

Of the ubiquitously expressed SFKs in RPE cells (Src, Yes, and Fyn), Fyn is the most divergent in terms of sequence, which may potentially account for a reduced sensitivity to Dasatinib compared to c-Src and/or Yes (Huang et al., 2016). To examine the effect of Dasatinib on Fyn specifically, a distinct antibody that has been previously reported to preferentially recognize phospho-Fyn Y420, homologous to Y416 on c-Src, was obtained (Githaka et al., 2016). Using this antibody, I found that Fyn auto-phosphorylation was less sensitive to Dasatinib treatment than other SFKs (Figure 4.3A). In particular, this effect was most prominent when comparing treatment of RPE cells with the lowest dose $(50 \mathrm{nM})$ of Dasatinib, which resulted in the inhibition of other SFKs detected by phospho-Src Y416 (Figure 4.2), but not of phospho-Fyn 
Y420. The specificity of this antibody was confirmed through siRNA silencing of Fyn, which significantly reduced the signal detected by immunoblotting with this putative anti-phospho-Fyn antibody. Importantly, using this anti-phospho-Fyn antibody, I also found Fyn to be phosphorylated in response to EGF (Figure 4.3B). From this we can infer that Fyn is selectively phosphorylated in response to EGF stimulation and that Fyn may be less sensitive to Dasatinib than other SFKs. Therefore, Fyn could be the SFK required for EGF-stimulated Akt phosphorylation in RPE cells.

To determine whether this is indeed the case and if Fyn is required for EGF-stimulated Akt phosphorylation, Fyn was silenced using siRNA in RPE cells and signaling downstream of EGFR was assessed. Fyn knockdown significantly reduced phosphorylation of Akt and Gab1 following EGF stimulation (Figure 4.3C), confirming a requirement for this SFK in EGFR signaling. I also determined that knockdown of the clathrin-binding Fyn adaptor protein TOM1L1, which is able to associate with EGFR and bind clathrin directly, significantly reduces Akt phosphorylation following EGF stimulation, outlining a role for TOM1L1 in EGFR signaling as well (Figure 4.4). Phosphorylation of TOM1L1 following EGF stimulation has been reported to exhibit a similar dose-dependent response to that of Akt phosphorylation (Liu et al., 2009), however this is the first time a functional link between TOM1L1 and Akt phosphorylation has been demonstrated. Importantly, the effects of Fyn or TOM1L1 knockdown were not due to changes in the receptor's activation as EGF-stimulated phosphorylation of EGFR Y1068 was unaffected (Figure 4.6A-B), or due to alterations in receptor availability for ligand, as there was no change in total cell surface EGFR as probed using an antibody that selectively recognizes the EGFR ectodomain (Figure 4.6C). From this we can infer that Fyn and TOM1L1 are required for EGF-stimulated Akt phosphorylation at a step in the PI3K/Akt pathway downstream of receptor 
activation. Since phosphorylation of Gab1 is decreased following Fyn siRNA, it can also be inferred that Gab1 phosphorylation is the most receptor proximal signaling event that demonstrates Fyn dependence in the PI3K/Akt pathway (Figure 4.3B), mirroring that of Gab1's dependence on clathrin previously reported (Garay et al., 2015). Together, perturbation of Fyn, TOM1L1, and clathrin have a similar effect on EGF-stimulated Akt phosphorylation, suggesting that these proteins may be working together at plasma membrane clathrin structures to control EGFR signaling.

Given that TOM1L1, Fyn, and clathrin are required for EGF-stimulated Akt phosphorylation, and that TOM1L1 interacts with Fyn and clathrin directly, it was next important to ask whether these interactions are required for Akt phosphorylation downstream of EGFR activation. Mutants of TOM1L1 defective in clathrin-binding (clathrin-binding mutant: ${ }^{447} \mathrm{FDPL}^{450}$ to ${ }^{447} \mathrm{AAAA}^{450}$ ) and Fyn-binding (Y460F) were used (Liu et al., 2009) in a dominantinterfering manner in RPE cells. Cells transfected with wild-type TOM1L1 exhibited an increase in EGF-stimulated Akt phosphorylation, however cells similarly transfected with clathrinbinding or Fyn-binding mutants of TOM1L1 failed to elicit an increase in Akt phosphorylation following EGF-stimulation (Figure 4.5). Taken together, TOM1L1 and its recruitment of Fyn into CCPs is required for EGF-stimulated Akt phosphorylation, demonstrating a role for TOM1L1 in bridging the clathrin endocytic machinery and EGFR to the kinase activity of Fyn.

\subsection{Regulation of PI3K/Akt signaling by Ack1}

Although these findings suggest a role for Fyn as a more Dasatinib insensitive kinase than other SFKs, and implicates a role for Fyn and TOM1L1 in Akt phosphorylation, this does not rule out the possibility that another cytosolic kinase may play a role in the pathway as well. In 
determining additional cytosolic kinases that may regulate clathrin-dependent Akt phosphorylation, I required the candidate protein to be able to associate with clathrin or the endocytic machinery directly and furthermore to have been previously implicated in RTK signaling. With this criteria, the NRTK Ack1 was identified as a strong candidate. Briefly, Ack1 was first identified as a Cdc42 effector protein (Manser et al., 2011), has since been found to form a complex with Grb2 to regulate EGFR signaling (Galisteo \& Yang, 2006; Shen et al., 2007), and binds the $\beta$-propeller domain of $\mathrm{CHC}$ directly using two unique motifs (Shen et al., 2011). Similarly to TOM1L1 and Fyn, I determined using siRNA gene silencing that Ack1 is required for EGF-stimulated Akt phosphorylation in RPE cells (Figure 4.7A). That both a requirement for Fyn and Ack1 in EGF-stimulated phosphorylation of Akt exists in RPE cells suggests two possible mechanisms of action, where i) Fyn and Ack1 are acting in parallel pathways, reflecting partial redundancy of these two kinases in Akt activation or ii) Fyn and Ack1 are acting within the same pathway, perhaps sequentially, to exert regulation on Akt. In order to differentiate between these two possibilities, I performed siRNA gene silencing of Fyn and Ack1 in combination and probed Akt activation downstream (Figure 4.7B). Ultimately, perturbation of both Fyn and Ack1 in RPE cells did not have an additive effect on inhibiting EGF-stimulated Akt phosphorylation, as quantification of Akt phosphorylation exhibited a similar inhibition as that of either Fyn or Ack1 perturbation alone. This suggests that Ack1 and Fyn may work sequentially as part of the same pathway downstream of EGFR that activates Akt, rather than working in separate pathways and both contributing to activation separately. If the latter were the case, siRNA knockdown of both proteins would inhibit Akt activation equivalently to inhibition of either alone added together. Consistently, the $\mathrm{SH} 2$ and SH3 domains of c-Src have been found to interact with Y518 and residues 623-652 on Ack1, 
respectively (Chan et al., 2011). Through this interaction Src was found to directly phosphorylate Y284 in the activation loop of Ack1, ultimately controlling its activity (Chan et al., 2011). Although Src was found to bind Ack1 best, other SFK members Lyn, Hck1, and Yes also coimmunoprecipitated with Ack1, suggesting that other SFKs than the ones examined here could also interact with Ack1, such as Fyn (Chan et al., 2011). A potential mechanism for Ack1 and Fyn working as part of the same pathway therefore requires that Fyn is activated in response to EGF stimulation (Figure 4.3), and in turn phosphorylates and activates Ack1 downstream, leading to downstream control of EGF-stimulated Akt phosphorylation.

In the continuation of this work in the future, it is important to delineate the signaling events leading to phosphorylation of Fyn and Ack1, as I have shown that they are both important signaling regulators downstream of EGFR activation. Since Fyn and Ack1 may be working in the same pathway, and since other SFKs have been demonstrated to phosphorylate the activation loop of Ack1, determining if and how Fyn phosphorylates Ack1 is of interest. To do this, first of all an antibody that recognizes the Y284 site of Ack1 would have to be obtained to probe endogenous activation of Ack1 following perturbation of Fyn. To determine whether Fyn works upstream of Ack1 in the EGF-stimulated Akt pathway, I would first perturb Fyn in RPE cells using siRNA and probe the phosphorylation of Ack1 following EGF stimulation. In addition to this, it would be useful to obtain a mutant construct of Fyn in which the kinase domain has been modified and inactivated (or "kinase dead"), or a mutant of Ack1 where Y518 and residues 623652 that have been previously shown to bind to Src are altered (ex. Y518F). Following siRNA silencing of endogenous Fyn or endogenous Ack1, I would transfect RPE cells with either the kinase dead Fyn, or mutants of Ack1 unable to bind Src and probe phosphorylation of i) Ack1 and ii) Akt. 
Following this, and if Fyn is responsible for phosphorylating and activating Ack1, it would next be important to probe the localization of both of these proteins following EGF stimulation. To do this, and similarly to the experiments presented in this thesis, I would either transfect RPE cells with the lowest detectable levels of eGFP-Fyn or eGFP-Ack1, or endogenously immunofluorescently stain these two proteins and determine whether they are localized to the same structures on the plasma membrane using TIRF-M. Lastly, an alternative technique to directly quantify the potential interaction of these two proteins would be using the technique of fluorescence resonance energy transfer (FRET), which can measure the proximity of two proteins within 8-10 $\mathrm{nM}$ of separation sensitively and more effectively than co-localization microscopy (De Angelis, 2004; Ouyang et al., 2019). Briefly, FRET relies on energy transfer between a donor and an acceptor fluorophore located on two different proteins in close proximity. If the two proteins of interest are found within 8-10 nM apart following initial excitation of the donor fluorophore, the acceptor fluorophore will be close enough to the donor fluorophore to accept an electron transfer that excites the acceptor fluorophore as well.

Just because these two proteins may be determined to interact, however, does not necessarily mean that a phosphorylation event is occurring, therefore it is important that these experiments described above are used in combination to complement one another and ultimately determine whether Fyn may act upstream of Ack1 in EGFR signaling.

In summary, I have elucidated a mechanism by which the SFK Fyn specifically may regulate EGFR signaling and Akt phosphorylation in a TOM1L1-dependent manner following EGF stimulation. Additionally, I have explored the possibility that additional cytosolic kinases play a role in clathrin-dependent signaling and determined that the clathrin-binding EGFRregulating kinase Ack1 is similarly required for EGF-stimulated Akt phosphorylation. This 
however doesn't exclude the possibility that there are other cytosolic kinases that influence EGFstimulated Akt phosphorylation, and that may be responsible for phosphorylation of Gab1 other than the ones tested here. The future directions of this research therefore include larger scale screens of clathrin-interacting proteins involved in endocytosis and signaling therefrom.

\subsection{Heterogeneity of TOM1L1/Fyn function in EGFR signaling for different cell types}

To determine whether the requirement for Fyn and TOM1L1 in EGFR signaling is reflected in cell lines other than RPE cells, I looked to several breast cancer cells lines (luminalA breast cancer subtype cells T47D, triple negative breast cancer cells BT-20, and Basal-B breast cancer subtype cells SUM149PT) as well as one additional non-transformed epithelial cell line MCF10A, a widely used in vitro model for studying normal breast cell function and transformation (Barnabas \& Cohen, 2013; Qu et al., 2015). Perturbation of Fyn or TOM1L1 in T47D, BT-20, and SUM149PT cells did not significantly reduce Akt phosphorylation, suggesting that in these various cancer cell lines, Akt activation downstream of EGFR occurs by some alternative mechanism that does not require TOM1L1 or Fyn. This is consistent with findings that both HeLa cells and RPE cells stably expressing ErbB2 are insensitive to clathrin perturbation and activate EGFR downstream of Akt readily following EGF stimulation (Garay et al., 2015). SUM149PT cells express ErbB2, providing a possible explanation as to why in these cells, and similarly to RPE cells expressing ErbB2, clathrin is not required for EGF-stimulated Akt phosphorylation (Barnabas \& Cohen, 2013).

In contrast to this however, EGF-stimulated Akt phosphorylation was significantly reduced following TOM1L1 or Fyn knockdown in the normal (non-malignant) mammary gland epithelial cell line MCF10A. It is possible then that the requirement of clathrin as a signaling scaffold may 
be a requirement in normal, non-transformed cells, while transformed cells forgoe this mechanism of Akt activation, potentially through mutations they have gained through transformation. An important outcome of this research in the future, and as discussed in section 6.1, therefore becomes determining how changes in these transformed cells allow them to activate signaling downstream of EGFR without the requirement for clathrin, Fyn, or TOM1L1, and whether these changes are innate or gained.

\subsection{A subset of clathrin structures contain EGFR, phospho-Gab1, Ack1, Fyn, and TOM1L1}

There are several lines of evidence that suggest the internalization of receptors from the plasma membrane via $\mathrm{CME}$ requires functionally distinct endocytic adaptor proteins that link them to the clathrin endocytic machinery. The uptake of TfR and low density lipoprotein receptor (LDLR) occurs constitutively even in the absence of ligand, and is dependent on sorting signals in their cytoplasmic tails that allow them to bind AP2, or the endocytic adaptor proteins Dab2 and ARH, respectively (Brodsky, 1988; Kirchhausen et al., 2014; Kirchhausen, Bonifacino, \& Riezman, 1997; Mettlen et al., 2010a). The GPCR family of receptors require $\beta$ arrestin proteins as adaptors for CME (Kirchhausen et al., 2014). Additionally, different receptors display different requirements for AP2 in CME; AP2 is seemingly dispensable for internalization of EGFR but is required for internalization of TfR (Motley et al., 2003; Pascolutti et al., 2019). In contrast, changes in phospholipase $\mathrm{C} \gamma 1$ (PLC $\gamma 1)$, intracellular $\mathrm{Ca}^{2+}$, or protein kinase C (PKC) inhibit EGFR internalization but not TfR (Delos Santos et al., 2017). It is therefore likely that distinct subsets of CCPs are specialized for the internalization of certain receptors. It follows from this that either specific sets of adaptor proteins and signaling effectors 
are enriched in specific CCPs following receptor recruitment, or that alternatively a heterogeneous initial formation of CCPs creates functionally distinct structures that can then each recruit a restricted subset of receptors following their engagement of ligand.

By labeling TfR and EGFR with fluorescently conjugated ligands, I have demonstrated that these two receptors localize largely to distinct populations of CCPs within the plasma membrane, and this segregation is not dependent on ligand availability as an increase in EGF concentration does not alter this finding (Figure 5.1). We find that CCPs harboring either receptor on their own are detected three times as frequently as CCPs harboring both receptors together, and this phenomenon is consistent across the ventral, middle, and dorsal areas of the cell as imaged using spinning-disk confocal microscopy (Figure 5.1D-F). This finding is consistent with previous studies which employed similar methods of imaging CCPs using fluorescently conjugated EGF and Tfn (Tosoni et al., 2005), however the findings of this study were not quantitatively assessed as were the experiments in this thesis. Therefore, our finding represents a quantitative, novel finding that whereby EGFR and TfR are found largely in distinct CCP populations that potentially support endocytosis of different receptors through distinct mechanisms.

In addition to $\mathrm{CCP}$ heterogeneity in regards to receptor content, $\mathrm{CCPs}$ are heterogeneous in their endocytic accessory and signaling effector content. I determined that Fyn is found within a subset of clathrin structures at the plasma membrane, and its enrichment is enhanced upon EGF stimulation (Figure 5.2, Figure 5.7, Figure 5.8). Importantly, Fyn's enrichment was not due to random overlap in the images as the EGF-stimulated gain in enrichment was lost upon scrambling the orientation of the channels to randomize the relative position of Fyn and clathrin channels (Figure 5.2A and Figure 5.7). Additionally, silencing of TOM1L1 abolished the EGF- 
stimulated gain in Fyn enrichment within clathrin structures (Figure 5.7). Furthermore, Fyn enrichment within clathrin structures was also observed at higher resolution using SIM (Figure 5.2B), which also further revealed that the centre of mass of Fyn and clathrin objects precicely overlap, suggesting that these are part of the same structure and not just adjacent to one another.

Despite this significant and specific enrichment of Fyn in CCPs following EGFstimulation, the quantified gain in Fyn recruitment to clathrin structures was only 50\% when examining ensemble averages across all CCPs in the EGF-stimulated cells (Figure 5.2A, Figure 5.7), suggesting either that low levels of Fyn are recruited broadly to all CCPs on the plasma membrane following EGF-stimulation or that Fyn is selectively and potently recruited to a subset of CCPs. Given that I found EGFR to be enriched within a subset of CCPs distinct from CCPs that house TfR, and that Fyn is required for EGF-stimulated Akt phosphorylation downstream of EGFR, it is possible that to mediate signaling downstream of EGFR, Fyn is specifically recruited to the population of CCPs housing EGFR. To examine whether the population of CCPs that contain EGFR also preferentially contain Fyn, clathrin structures were immunofluorescently labelled in cells transfected with the lowest detectable levels of eGFP-Fyn and stimulated with rhodamine-conjugated EGF. CCPs were sorted into cohorts dependent on whether they contained EGF or not (EGF+ or EGF-) based on an arbitrary but systematic threshold for EGF, as we have done previously (Delos Santos et al., 2017; Lucarelli et al., 2017). The cohort of CCPs containing EGF exhibited significantly more Fyn enrichment than those that did not contain EGF (Figure 5.8D-F). Thus, the CCPs that harbor EGFR are different than those that contain other receptors such as TfR, and part of the reason for these differences is that they preferentially recruit Fyn to them following EGF stimulation. 
Also using TIRF-M coupled to automated detection and analysis, TOM1L1 (Figure 5.3), phospho-Gab1 (Figure 5.4, Figure 5.5), and Ack1 (Figure 5.6) were found to be enriched within a subset of CCPs at the plasma membrane, further demonstrating the heterogeneity of CCPs. Interestingly, the enrichment of phospho-Gab1 within a subset of CCPs was found following stimulation with either EGF or HGF, supporting the finding from Chapter 3 that clathrin is required for signaling by each of these receptors and suggesting that clathrin acts as a signaling scaffold, forming a signaling nanodomain enriched with signaling regulators required by both EGFR and Met receptor. Given that Gab1 is able to directly bind Met receptor (Weidner et al., 1996), and is a key signaling intermediate in pathways downstream of receptor activation, it is not surprising that Gab1 is found localized in a subset of CCPs. Similarly to that of Fyn, I also aimed to determine whether the subset of CCPs that contained EGFR also preferentially contained Ack1. Following stimulation with A555-EGF or A647 Tfn to label EGFR and TfR respectively, cells transfected with eGFP-Ack1 were found to be robustly and significantly enriched within EGFR positive CCPs. These results together further support the heterogeneity of CCPs and suggest a concentration of signaling effectors in CCPs that contain EGFR.

While these findings provide evidence that these proteins are enriched within a subset of clathrin structures, and that Fyn and Ack1 are preferentially found within CCPs that contain EGFR, an important future direction of this research becomes identifying whether Fyn, TOM1L1, Ack1, and/or phospho-Gab1 are recruited to the same clathrin structures at the plasma membrane following ligand stimulation, and whether these structures are unique from those devoid of these proteins. To do this, the enrichment of each protein would ideally be fluorescently determined using endogenous levels of each protein tagged with different fluorophores and coupled to imaging by TIRF-M. I expect that since TOM1L1 and Fyn are 
known interactors, and since Fyn binding and clathrin binding by TOM1L1 are required for EGF-stimulated Akt activity, I would see Fyn and TOM1L1 localized to the same clathrin structures on the plasma membrane. If Ack1 is acting downstream of Fyn in the same pathway leading to EGF-stimulated Akt phosphorylation, I would expect that Ack1 is also found localized to the same clathrin structures as Fyn and TOM1L1.

Interestingly, TOM1L1 is found within a subset of clathrin structures on the plasma membrane in both the presence and absence of EGF (Figure 5.3), however, Fyn only becomes significantly enriched within clathrin structures following EGF stimulation (Figure 5.2) in a TOM1L1-dependent manner (Figure 5.7). This suggests that TOM1L1 is resident within a subset of clathrin structures constitutively, and Fyn is recruited into CCPs in a TOM1L1dependent manner following EGF stimulation. Consistent with this, TOM1L1 is phosphorylated by Fyn at Y460 following EGF stimulation, an effect which is abolished when cells are treated with the SFK inhibitor PP2 or when a Y460F mutant of TOM1L1 is expressed (Liu et al., 2009; Seykora et al., 2002b). This phosphorylation site is also part of a motif responsible for then binding the SH2 domain of Fyn only once phosphorylated, highlighting the reciprocal regulatory relationship between TOM1L1 and Fyn (Seykora et al., 2002). Phosphorylation of TOM1L1 is also required for its interaction with the $\mathrm{p} 85$ regulatory subunit of PI3K and with Grb2 (Seykora et al., 2002), however it is not required for binding clathrin (Collin et al., 2007; Katoh et al., 2006). This suggests that in the absence of EGF stimulation, TOM1L1 may still associate with clathrin. In addition to its requirement in recruiting Fyn to a subset of clathrin structures, TOM1L1 binding enhances the activity of Fyn (Li et al., 2005). Consistent with this, I have found that Fyn (Figure 4.3A), but not c-Src (Figure 4.1), is phosphorylated and active in response to EGF stimulation in RPE cells, supporting a potential mechanism by which TOM1L1 
in clathrin structures becomes phosphorylated by Fyn at Y460 following EGF stimulation, which reciprocally creates a binding site for Fyn, recruiting it into CCPs and enhancing its kinase activity.

Notably, Fyn is unable to associate directly with clathrin or EGFR, therefore TOM1L1 is effectively bridging their interaction by binding clathrin and allowing Fyn to participate in receptor proximal signaling at the level of the CCP. This is further supported by results in Figure 4.5 which show that both clathrin and Fyn binding mutants of TOM1L1 fail to elicit EGFstimulated Akt phosphorylation. Fyn may thus regulate clathrin-dependent signaling in two general ways: 1) direct phosphorylation of signaling intermediates in PI3K signaling, such as Gab1, effecting clathrin-dependent control over this signaling pathway or 2) by phosphorylating other structural components of CCPs, which in turn leads to formation of signaling-competent clathrin signaling structures that can regulate PI3K activation by other kinase(s). I discuss these possibilities next.

Due to its dual lipidation, Fyn is found primarily at the plasma membrane within detergent-insoluble lipid rafts (Gottlieb-Abraham et al., 2016; Resh, 1994; Davy et al., 1999; Pereira \& Chao, 2007). Recently, these lipid rafts have been found to provide a suppressive microenvironment for Fyn regulation, as its localization outside of lipid rafts leads to hyperphosphorylation and activation of Fyn in mouse embryonic fibroblasts (Ouyang et al., 2019). Given that my findings indicate that Fyn is enriched within a subset of CCPs following EGF stimulation, and that there is little Fyn found enriched within CCPs in unstimulated cells, it follows that this could be due to Fyn suppression within lipid rafts. Disruption of lipid rafts has been reported to enhance EGFR and MAPK phosphorylation (Mineo et al., 1999; Ringerike et al., 2002; Roepstorff et al., 2002), indicating that control of EGFR signaling by cholesterol- 
enriched membrane microdomains may be due to suppressed activity of signaling effectors contained there in. In the future to test this hypothesis, disruption of lipid rafts at the plasma membrane can be achieved using drugs such as Nystatin (Baek et al., 2013). By sequestering cholesterol at the plasma membrane, Nystatin can effectively disrupt interactions keeping Fyn within this microdomain potentially leading to its hyperactivation in RPE cells. To test this, an antibody fit for immunofluorescent staining that recognizes endogenous phosphorylated Fyn can be used to probe the activation and localization of Fyn following cholesterol sequestration. It would also be interesting to determine whether Nystatin treatment led to an increase in the enrichment of Fyn within CCPs, both in the presence or absence of ligand (EGF). Additionally, it would be of interest to treat RPE cells with Nystatin or a similar agent and probe the downstream activation of signaling pathways following EGFR phosphorylation.

SFKs have been shown to phosphorylate clathrin, AP2, and Dynamin in response to EGF stimulation, however the mechanism and role of some of these phosphorylation events remains unclear (Wilde et al., 1999; Zimmerman et al., 2009). SFK-mediated phosphorylation of CHC results in longer-lived, more stable CCPs at the plasma membrane (Bonazzi et al., 2011). The SFK-mediated phosphorylation of the $\beta$-subunit of AP2 has been demonstrated to occur following EGFR activation, and to be required for the clathrin-mediated internalization of many GPCRs including the Angiotensin Type 1, $\beta 2$-adrenergic, and endothelin A receptors as it promotes association of AP2 with the GPCR endocytic adaptor protein $\beta$-arrestin (Zimmerman et al., 2009). In addition, EGFR activation also stimulates Src-dependent phosphorylation of CHC, and cells lacking c-Src and therefore clathrin phosphorylation display modest impairment of EGF internalization (Wilde et al., 1999). Another study also demonstrating the role of SFKs in phosphorylation of CHC finds that this effect is abrogated by depletion of TOM1L1 (Collin et 
al., 2007). Since SFKs share often redundant function in cells, the modest impairment of EGF internalization in cells lacking c-Src in this study may be due to compensation by other SFKs in the cell, including Fyn. This is consistent with a role for Fyn, but likely not c-Src (given the effects of Dasatinib treatment) that I observed in the control of EGFR signaling leading to PI3KAkt signaling. As such, in RPE cells, Fyn may be influencing the phosphorylation of clathrin and AP2 following TOM1L1-dependent recruitment to CCPs, thus controlling clathrin structure dynamics, which in turn may impact PI3K activation. Given the complexity of receptor-mediated endocytosis, understanding the mechanism by which Fyn and TOM1L1 regulate clathrin and AP2 phosphorylation and influence CCP internalization is beyond the scope of this study; however, this represents an important area of future research.

Examining phosphorylation of CHC and AP2 in cells lacking Fyn and TOM1L1, or in cells expressing the clathrin and Fyn binding mutants of TOM1L1 would be of interest in future studies. Since SFKs have been shown to phosphorylate CHC directly, I would expect that inhibition of SFKs in RPE cells would decrease detectable CHC phosphorylation and influence clathrin assembly on the plasma membrane. Additionally, measuring the influence of Fyn or TOM1L1 perturbation on EGF internalization to determine if it exhibits the same influence as Src should be examined.

In summary, I have shown that both clathrin and Fyn binding mutants of TOM1L1 block EGF-stimulated Akt phosphorylation (Figure 4.5). From this, we can infer that Fyn recruitment to clathrin structures is dependent on TOM1L1, and that recruitment of TOM1L1 and Fyn to clathrin structures is critical for EGF-simulated Akt phosphorylation. Additionally, I find that Fyn and Ack1 are preferentially recruited to CCPs that contain EGFR following EGF stimulation. This evidence together further supports a model in which a subset of clathrin 
structures at the plasma membrane contain TOM1L1 that upon EGF stimulation recruit and bind Fyn leading to phosphorylation of Akt in a clathrin-dependent manner. Abolishing TOM1L1's interaction with Fyn or clathrin fails to recruit Fyn to CCPs and fails to elicit phosphorylation of Akt, suggesting that TOM1L1, Fyn, and clathrin together are creating a signaling capable plasma membrane structure distinct from CCPs that do not contain Fyn or TOM1L1.

\subsection{Clathrin structures that recruit Fyn or TOM1L1 have distinct properties}

The dynamics, size, composition and other properties of CCPs on the plasma membrane are highly heterogeneous. For instance, CCPs lifetimes range from 20 seconds to 2 minutes to form a cytosolic coated vehicle (Dinah Loerke et al., 2011). What ultimately determines the length of this process? CME and CCP dynamics can be regulated by cargo (Puthenveedu \& von Zastrow, 2006), cargo-adaptor interactions (Marcel Mettlen et al., 2010a), and even receptor clustering (Liu et al., 2010). CCPs can be categorized into three kinetically distinct subpopulations based on unique time constants representing their average time resident on the plasma membrane. There are two short-lived populations, both of which demonstrate time constants of less than 15 seconds of residency at the plasma membrane, are deemed early abortive and late abortive CCPs, and one long-lived subpopulation deemed the productive CCPs with a mean lifetime of approximately 120 seconds (Loerke et al., 2009; Mettlen et al., 2010, 2009). In BSC1 cells, productive CCPs constitute only $38 \%$ of total CCPs at the plasma membrane meaning that more than half of all CCPs at any given time are short-lived and abortive structures (Dinah Loerke et al., 2011, 2009). It is thought that aberrant structures not suited for completion become abortive, whereas productive CCPs stabilize and complete maturation and scission to form an endocytic CCV by completing some sort of endocytic 
checkpoint that may depend on endocytic accessory proteins, membrane curvature, and cargo recruitment. Although the majority of CCPs at a given time on the plasma membrane are shortlived and abortive, it remains to be determined whether these structures have any specialized function, or if instead these reflect only unsuccessful attempts at coat formation. Nonetheless, the presence of abortive structures, and perhaps of an active process to turn over abortive CCPs indicates that while the initial stages of CCP formation may be unregulated and have stochastic inputs, the formation of stable CCPs requires specific aspects of initial assembly to occur. Moreover, these aspects of initial CCP assembly provide interesting insight into the heterogeneity of CCPs themselves, including of lifetime, size and composition.

Through live cell imaging using TIRF-M coupled to automated detection and analysis, I have demonstrated that CCPs containing Fyn or TOM1L1 display unique lifetime, size, dynamic activity, and composition (Figure 5.11, Figure 5.11). The mean GFP-Fyn and GFP-TOM1L1 intensity was significantly higher in short-lived CCPs (10-40 seconds) (Figure 5.10D,I), and Fyn or TOM1L1 positive CCPs exhibited longer lifetimes (Figure 5.10C,H) and were significantly larger (in terms of clathrin recruitment) than those that did not contain either (Figure 5.10E,J). As the presence of Fyn or TOM1L1 resulted in larger CCPs and longer CCP lifetimes, directly relating to residence time at the plasma membrane, Fyn or TOM1L1 may be influencing the ability of a CCP to become productive and long-lived rather than abortive and/or short-lived. The presence of Fyn and TOM1L1 may be acting to stabilize CCPs that contain them, allowing their advancement through some still unknown checkpoint that allows a CCP to become productive. This finding adds to a pool of literature supporting the hypothesis that heterogeneity in CCP behavior reflects differences in their cargo and adaptor content (Merrifield et al., 2005; Mettlen et al., 2010b; Rappoport et al., 2005). 
Recently, phospho-Akt and the tumor suppressor PTEN have been found to be localized to and regulate dynamics of a subset of short-lived signaling capable CCPs that also contain EGFR (Rosselli-Murai et al., 2018). Through its lipid phosphatase activity, PTEN exerts these effects by hydrolyzing $\mathrm{PIP}_{3}$ to $\mathrm{PIP}_{2}$ following recruitment to the plasma membrane. In addition, loss of PTEN results in an accumulation of PIP 3 at the plasma membrane (Stambolic et al., 1998) and a higher proportion of short-lived CCPs with a higher initiation density (Rosselli-Murai et al., 2018). Activation of EGFR following ligand binding results in receptor phosphorylation leading to recruitment and phosphorylation of Gab1 (Holgado-Madruga et al., 1996; Lisa S. Lock et al., 2000; Mattoon et al., 2004b), and activation of PI3K. The Gab1-mediated PI3K activation causes a local increase in concentration of $\mathrm{PIP}_{3}$ following phosphorylation of plasma membrane $\mathrm{PIP}_{2}$ required for Akt phosphorylation downstream (Mattoon et al., 2004b). Gab1 also participates in a positive feedback loop here: the activation of PI3K and production of $\mathrm{PIP}_{3}$ at the plasma membrane itself recruits more Gab1 to the plasma membrane via its $\mathrm{PH}$ domain, in turn activating more PI3K leading to more $\mathrm{PIP}_{3}$ production and eventual Akt phosphorylation (Kiyatkin et al., 2006; Rodrigues et al., 2000; Vanhaesebroeck \& Alessi, 2000). In Figure 5.10, I demonstrate that the mean fluorescence intensity of Fyn and TOM1L1 is significantly higher in the 10-20 second cohort of CCPs grouped by lifetime than in longer lived CCPs. In line with the recruitment of PTEN and Akt to a subset of short-lived CCPs that are signaling-capable (Rosselli-Murai et al., 2018), Fyn and TOM1L1 are readily and significantly detected in shortlived structures following EGF stimulation (as well as longer-lived structures), suggesting that these proteins may be working in concert to regulate signaling from the plasma membrane in short-lived structures as well. 
Notably, SFKs have been shown to phosphorylate CHC directly in NIH 3 T3 cells, an effect abrogated by depletion of TOM1L1 (Collin et al., 2007). In T cells, which express SFKs other than the ubiquitous Src, Yes and Fyn, the SFK Lyn is responsible for CHC phosphorylation which is ultimately required for ligand-induced internalization of the T-cell receptor (Crotzer et al., 2004). This requirement has also been demonstrated for B-Cell receptor colocalization with and regulated internalization by clathrin (Stoddart et al., 2002). Phosphorylation of clathrin by SFKs ultimately may be controlling receptor internalization by modifying clathrin structural dynamics, as both Src and Fyn phosphorylate Y1477 within a CHC hub fragment that has been shown to interact with clathrin light chain and is generally thought to control clathrin assembly (Fletterick et al., 1999; Näthke et al., 1992; Wilde et al., 1999). Therefore, the unique behavior displayed by Fyn and TOM1L1 positive CCPs (Figure 5.10) and therefore signaling capable structures may be a result of Fyn-dependent phosphorylation of CHC following EGF-stimulated and TOM1L1-dependent recruitment of Fyn to CCPs that preferentially contain EGFR. This potential phosphorylation event may act to stabilize clathrin and promote assembly, leading to the production of the longer-lived, productive CCPs that contain Fyn and TOM1L1 at the plasma membrane that we see in Figure 5.10.

In addition to the possibility that Fyn phosphorylates clathrin directly and influences CCP stability and dynamics, Fyn-proximity and phosphorylation of clathrin at CCPs may promote the recruitment of a unique complement of endocytic accessory proteins to CCPs containing Fyn, or alternatively, could influence the phosphorylation of recruited endocytic accessory proteins. Indeed, we see altered protein composition in Fyn positive CCPs: clathrin structures that contain Fyn exhibit enhanced amphiphysin recruitment, and slightly decreased epsin recruitment compared to Fyn negative clathrin structures (Figure 5.11), suggesting a link between the 
recruitment of a unique complement of endocytic accessory proteins and unique lifetime and size of Fyn positive clathrin structures.

Overall, I have found here that CCPs that contain either Fyn or TOM1L1 exhibit unique life time, size, dynamic activity, and protein composition compared to CCPs devoid of these signaling regulators. The detected presence of Fyn and TOM1L1 in both short-lived, abortive CCPs and in long-lived, productive CCPs highlights a role for these proteins in potentially regulating clathrin dynamics through phosphorylation, or in altering recruitment of additional endocytic signaling effectors, as demonstrated with changes in epsin and amphiphysin. Together, these results confirm that clathrin structures that preferentially contain Fyn, TOM1L1, EGFR and other signaling effectors (Ack1, Gab1, PTEN, Akt) create a nanodomain from which clathrin acts a signaling scaffold, coordinating the activation of the PI3K/Akt pathway downstream of EGFR.

\subsection{Conclusion}

This thesis investigated the mechanisms by which clathrin regulates receptor signaling from the plasma membrane through the formation of clathrin signaling nanodomains. CCPs are highly dynamic and heterogeneous structures regulated by the cargo and adaptor proteins they recruit (Loerke et al., 2011; Mettlen et al., 2010a; Puthenveedu , 2006; Sorkin \& Puthenveedu, 2013). The ligand-dependent internalization of many RTKs at the plasma membrane represents a principal process regulating the duration and propagation of signaling, thereby preventing overstimulation that could potentially lead to cellular transformation. Indeed, amplification, mutation, and overexpression of ErbB family receptors are well known drivers of many forms of human cancers, most notably of the lung, breast, and colorectal subtypes, and for this reason this receptor family is targeted in many anticancer therapies to date (Roskoski, 2014). It is therefore 
important that we delineate the regulation of cellular signaling downstream of these receptors and generate a clear picture of signaling regulators to identify potential new drug targets for cancer research.

Previous findings from our lab have demonstrated that perturbation of clathrin, but not Dynamin 2, inhibits EGF-simulated Gab1 and Akt phosphorylation, outlining a novel role for clathrin in EGFR signaling, and not just endocytosis (Garay et al., 2015). Thus, clathrin scaffolds may represent unique plasma membrane signaling nanodomains required for signaling by certain receptors, a function that can be separated from vesicle formation. The mechanism by which clathrin may exert these effects on receptor signaling remains unclear, providing a major topic of investigation for my research.

My work elucidated that clathrin exerts control over signaling downstream of the Met receptor in addition to its regulation of EGFR signaling (Garay et al., 2015). Importantly, clathrin was not found to be required for Akt phosphorylation downstream of EGF stimulation in several breast cancer cell lines, as well as in RPE cells expressing ErbB2 as previous studies from our lab have shown, suggesting that these cells have developed alternative mechanisms of signaling downstream of EGFR. The requirement for clathrin however was replicated in the endothelial non-malignant cell line MCF10A. From this, we can infer that either these transformed cells have alternative mechanisms of stabilizing signaling-capable CCPs other than requiring TOM1L1/Fyn as RPE and MCF10A cells do, or that these cells forego the requirement for clathrin as a signaling regulator all together.

My work has also revealed new insights into CCP heterogeneity at the plasma membrane. Specifically, I find that EGFR and TfR are found largely in distinct populations of CCPs, and a select subset of endocytic signaling regulators (phospho-Gab1, Fyn, TOM1L1, and Ack1) are 
enriched within a subset of CCPs following EGF stimulation. Importantly, the subset of CCPs that contain EGFR are preferentially enriched with Fyn in an EGF- and TOM1L1-dependent manner, and also enriched in Ack1, and these structures exhibit unique lifetimes, size, dynamic activity, and protein composition.

Through this work, I have highlighted another way in which the plasma membrane and membrane nanodomains compartmentalize growth factor signaling leading to Akt activation. The cell may employ this mechanism as a way to filter noise and prevent aberrant activation of receptors contained here. As such, I propose that the selective recruitment of certain signaling proteins to a subset of clathrin coated pits imparts upon these structures the unique properties of a signaling nanodomain required to control receptor signaling downstream. 


\section{Appendix}
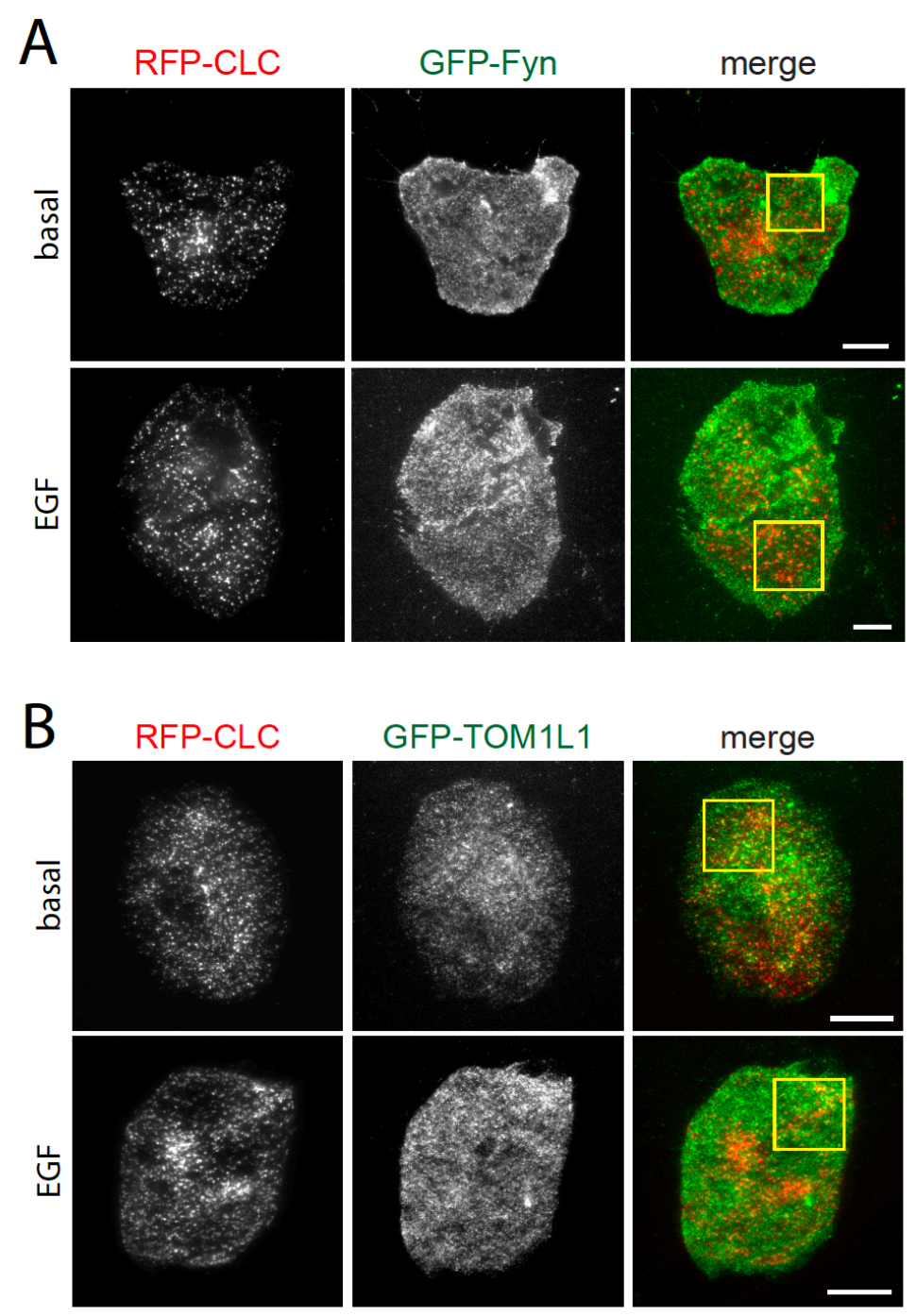

Figure A2 Full image panels of TIRFM fluorescence micrographs shown in Figure 5.2A and Figure 5.3.

Cell samples treated and prepared as in Figures 5.2A and 5.3 were subjected to TIRF-M. Shown here are the full-sized images of the representative images shown in Figured 5.2A and 5.3, with a yellow box showing the area enlarged, scale $=20 \mu \mathrm{m}$. 

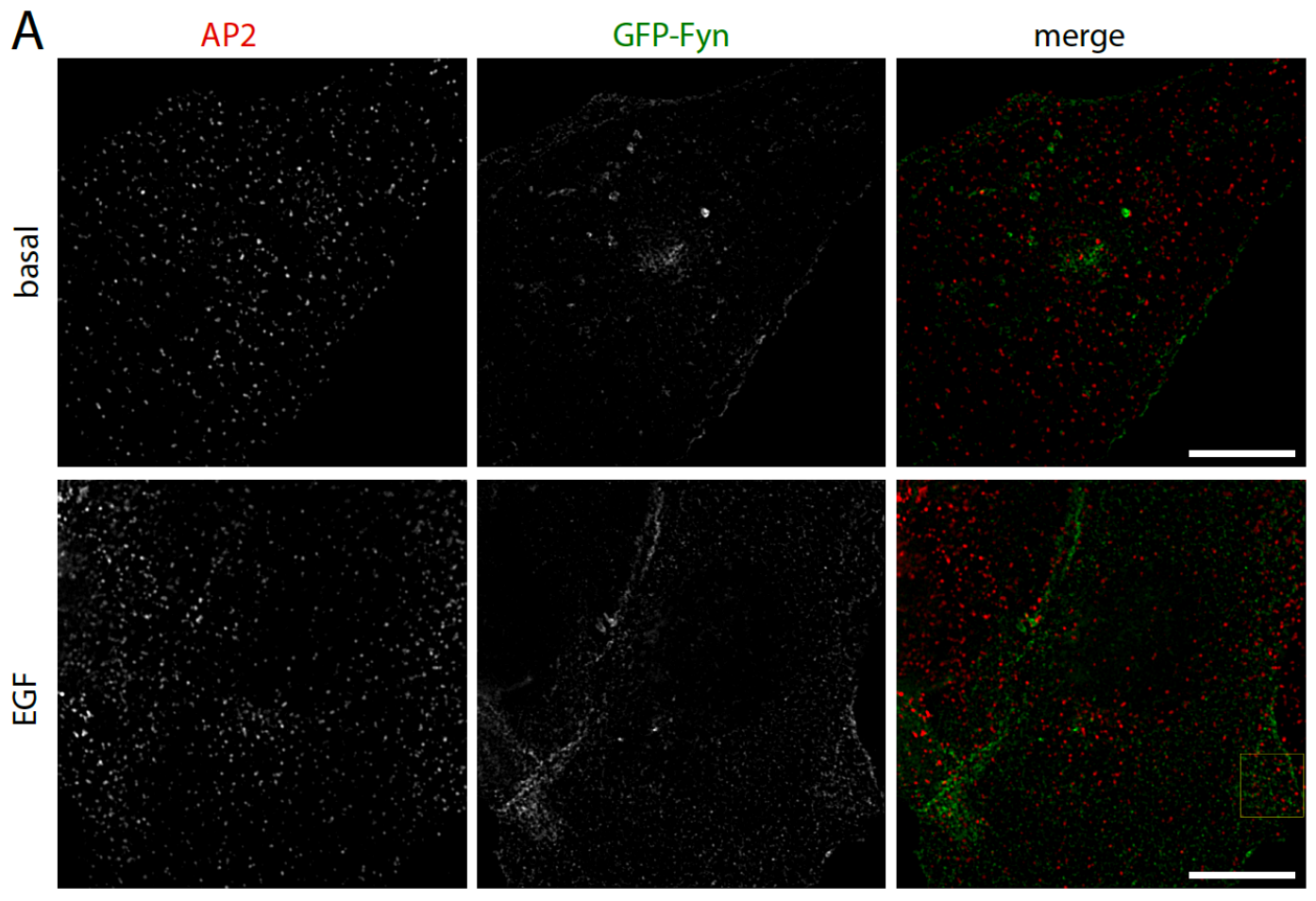

B
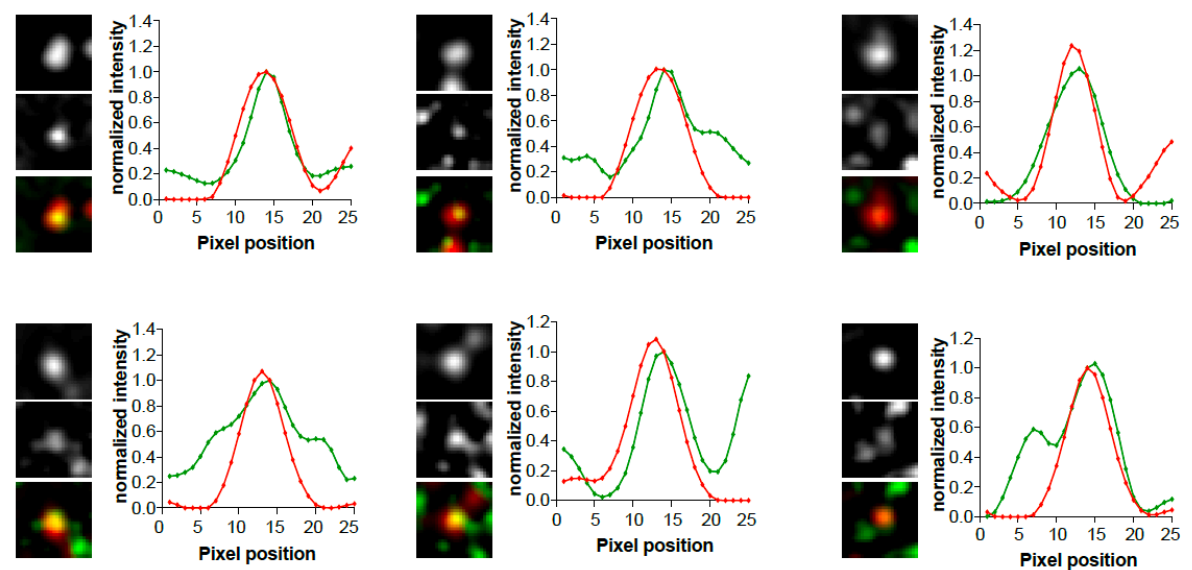

Figure A3 Full image panels of SIM fluorescence micrographs shown in Figure 5.2B.

Cell samples treated and prepared as in Figure 5.2B were subjected to SIM. (A) Shown here are the full-sized images of the representative images shown in Figure 5.2B. Scale $=10 \mu \mathrm{m}$. (B) Shown are line scan analysis of AP2 and eGFPFyn in individual clathrin structures (bottom panels). 

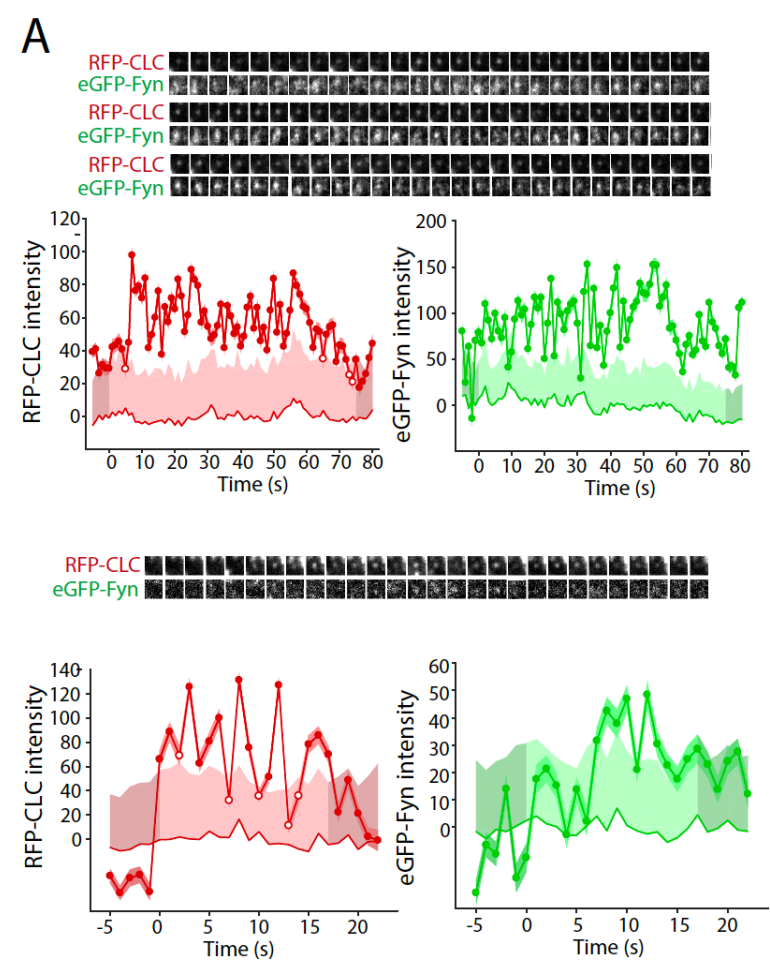

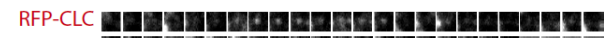

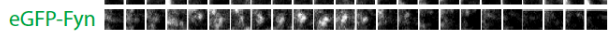
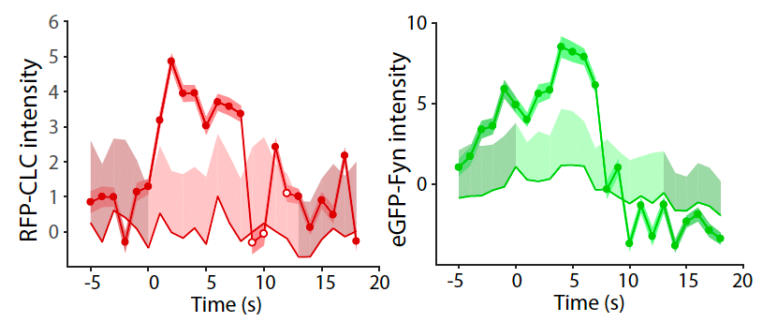

B
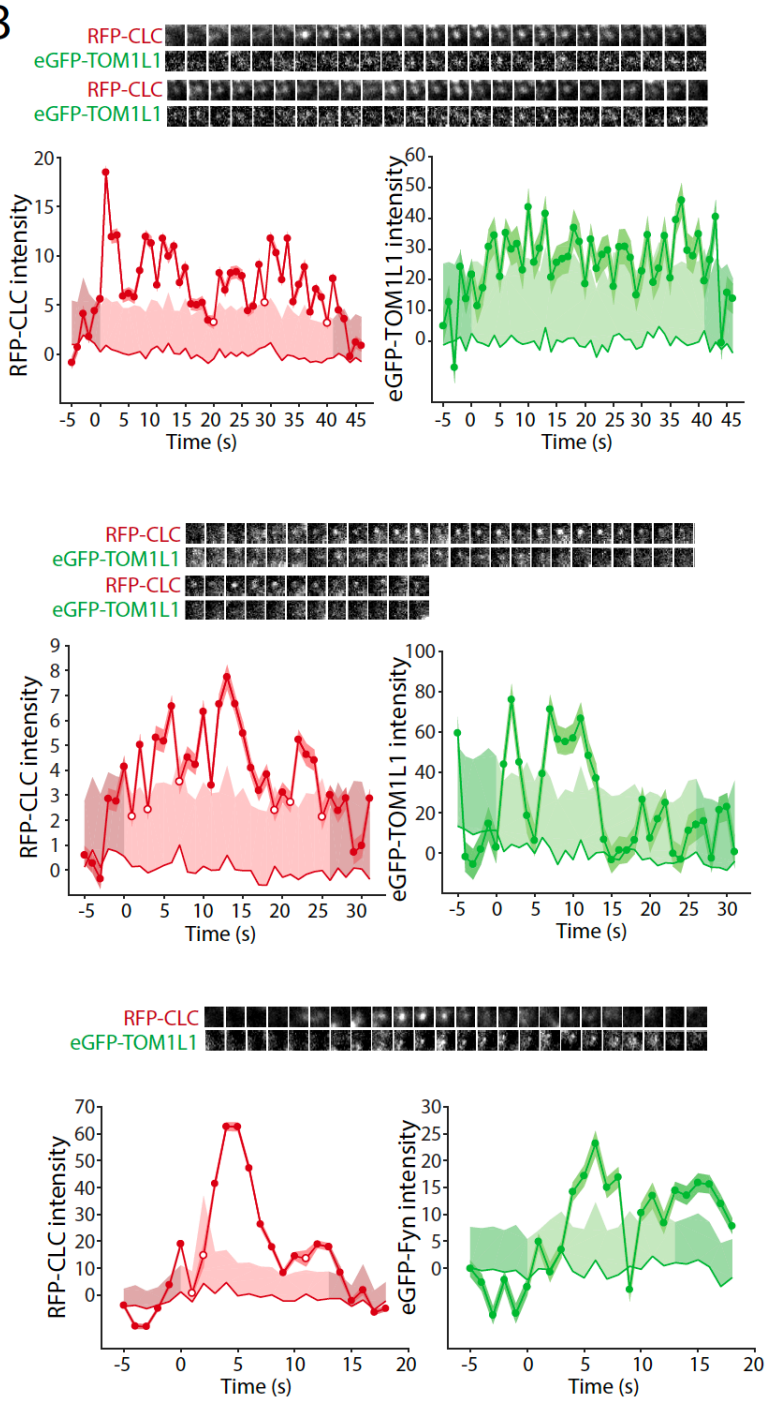

Figure A4 Additional representative traces of individual Fyn+ and TOM1L1+ CCPs.

Cell samples were treated, prepared and subjected to time-lapse TIRFM imaging as per Figure 6. Shown are additional representative Fyn+ (A) or TOM1L1+ (B) CCPs showing fluorescence images corresponding to clathrin and Fyn/TOM1L1 centered at the detected object (top panels), as well as quantification of eGFP-Fyn (A) or eGFP-TOM1L1 (B) and RFP-CLC within the object (bottom panels). 

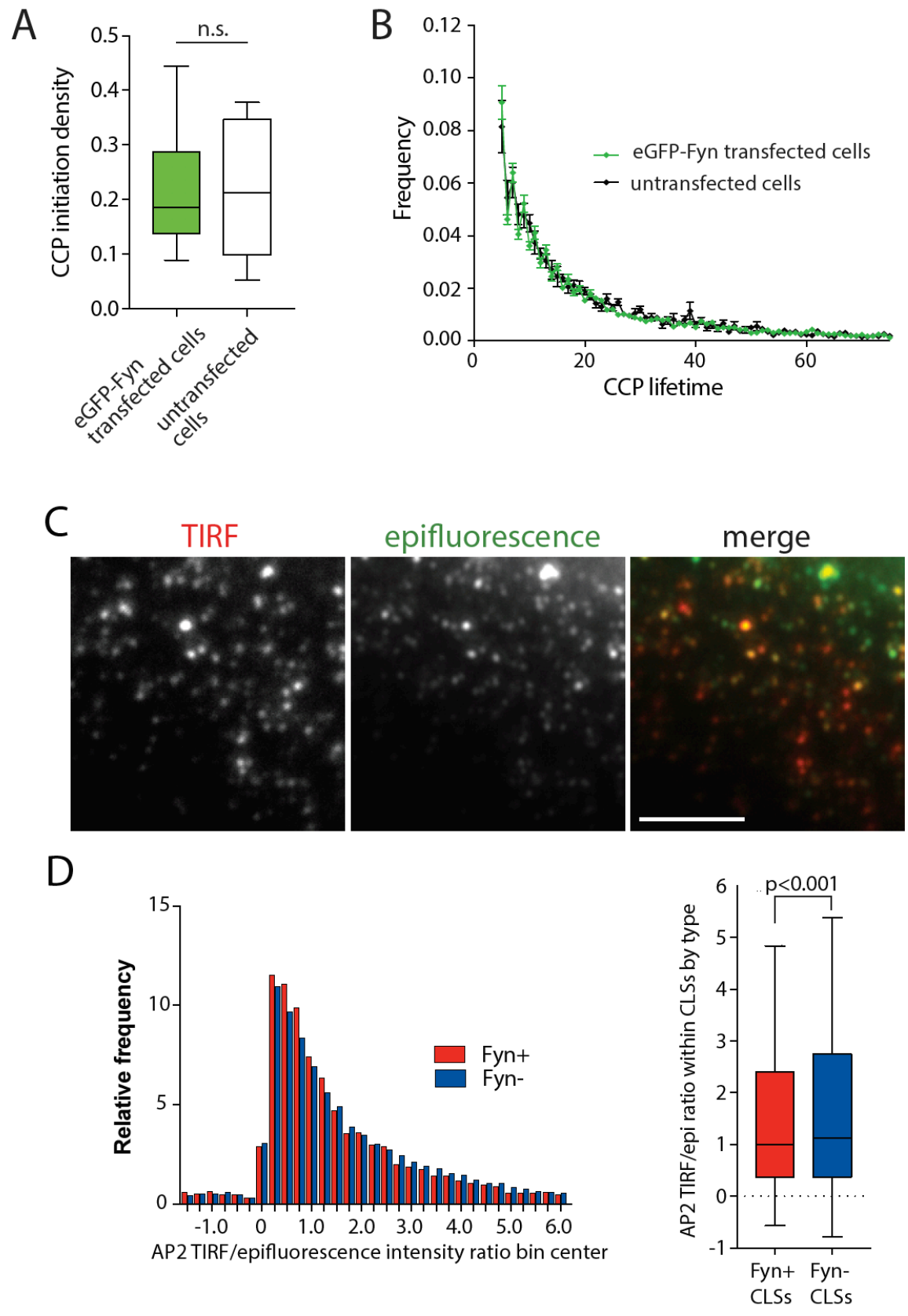

Figure A5 Expression of eGFP-Fyn under the conditions examined does not alter CCP population dynamics and analysis of curvature in Fyn+ clathrin-labelled structures.

(A-B) The TIRFM time-lapse image series of RPE cells stably expressing RFP fused to clathrin light chain (Tag-RFP-T-CLC) transfected with eGFP-Fyn (as in Figure 6A-E) was acquired alongside similar image series in RPE cells expressing only Tag-RFP-T-CLC (stably) but not eGFP-Fyn. Each time-lapse TIRFM dataset of cells expressing eGFP-Fyn or not transfected with eGFP-Fyn ('untransfected cells') was subjected to automated detection, tracking and analysis of 
CCPs. (A) The CCP initiation density, depicting median (bar) interquartile range (boxes) and full range (whiskers). (B) The frequency distribution of CCP lifetimes. The expression of eGFP-Fyn does not grossly alter the initiation density or lifetime distribution of the total cellular population of CCPs. The number of CCPs and cells analyzed, respectively, for each condition are as follows eGFP-Fyn expressing cells (18383 CCPs, 17 cells) and cells not expressing eGFP-Fyn (16412 CCPs and 6 cells). (C-D) Clathrin-labelled structures that recruit Fyn do not exhibit defects in curvature generation. RPE cells were transfected with eGFP-Fyn, stimulated with $20 \mathrm{ng} / \mathrm{mL}$ EGF for $5 \mathrm{~min}$, then fixed and subjected to immunofluorescence staining of AP2. Scale $5 \mu \mathrm{m}$. (A) Shown are representative images of AP2 staining detected by TIRF and widefield epifluorescence microscopy. Micrographs obtained by TIRFM were subjected to automated detection and analysis of clathrin structures, allowing quantification of each protein within each CLS (detected via AP2). (B) Shown (left panel) is the frequency distribution of the ratio TIRF to widefield epifluorescence signal intensity of each CLS within a representative experiment, sorted into Fyn+ or Fyn- CLSs cohorts. Also shown (right panel) is the ensemble average of the ratio TIRF to widefield epifluorescence signal intensity of each CLS, sorted into Fyn+ or Fyn- CLSs cohorts. These results are depicted as median (bar) 25th/75th percentiles (boxes) and whiskers (full range). The number of CLSs and cells analyzed, respectively, is 65,921 and 115 cells, taken from 3 independent experiments. 


\section{References}

Abram, C. L., \& Courtneidge, S. a. (2000). Src family tyrosine kinases and growth factor signaling. Experimental Cell Research, 254(1), 1-13. https://doi.org/10.1006/excr.1999.4732

Aguet, F., Antonescu, C. N., Mettlen, M., Schmid, S. L., \& Danuser, G. (2013a). Advances in analysis of low signal-to-noise images link dynamin and AP2 to the functions of an endocytic checkpoint. Developmental Cell, 26(3), 279-291. https://doi.org/10.1016/j.devcel.2013.06.019

Aguet, F., Antonescu, C. N., Mettlen, M., Schmid, S. L., \& Danuser, G. (2013b). Advances in Analysis of Low Signal-to-Noise Images Link Dynamin and AP2 to the Functions of an Endocytic Checkpoint. Developmental Cell, 26(3), 279-291. https://doi.org/10.1016/j.devcel.2013.06.019

Akbarzadeh, S., Ji, H., Frecklington, D., Marmy-Conus, N., Mok, Y. F., Bowes, L., ... Dorow, D. S. (2002). Mixed lineage kinase 2 interacts with clathrin and influences clathrin-coated vesicle trafficking. Journal of Biological Chemistry, 277(39), 36280-36287. https://doi.org/10.1074/jbc.M204626200

Alessi, D. R., \& Cohen, P. (1998). Mechanism of activation and function of protein kinase B. Current Opinion in Genetics and Development. https://doi.org/10.1016/S0959-437X(98)80062-2

Alvarado, D., Klein, D. E., \& Lemmon, M. A. (2010). Structural Basis for negative cooperativity in growth factor binding to an EGF receptor. Cell. https://doi.org/10.1016/j.cell.2010.07.015

Anderson, K. E., Kielkowska, A., Durrant, T. N., Juvin, V., Clark, J., Stephens, L. R., \& Hawkins, P. T. (2013). Lysophosphatidylinositol-Acyltransferase-1 (LPIAT1) Is Required to Maintain Physiological Levels of PtdIns and PtdInsP2 in the Mouse. PLoS ONE, 8(3), 1-14. https://doi.org/10.1371/journal.pone.0058425

Antonescu, C. N., Aguet, F., Danuser, G., \& Schmid, S. L. (2011). Phosphatidylinositol-(4,5)bisphosphate regulates clathrin-coated pit initiation, stabilization, and size. Molecular Biology of the Cell, 22(14), 2588-2600. https://doi.org/10.1091/mbc.e11-04-0362

Antonny, B., Burd, C., De Camilli, P., Chen, E., Daumke, O., Faelber, K., ... Schmid, S. (2016). Membrane fission by dynamin: what we know and what we need to know. The EMBO Journal. https://doi.org/10.15252/embj.201694613

Bae, Y. S., Kang, S. W., Seo, M. S., Baines, I. C., Tekle, E., Chock, P. B., \& Rhee, S. G. (1997). Epidermal Growth Factor (EGF)-induced Generation of Hydrogen Peroxide. Journal of Biological Chemistry. https://doi.org/10.1074/jbc.272.1.217

Baek, S., Kim, S. M., Lee, S. a, Rhim, B. Y., Eo, S. K., \& Kim, K. (2013). The Cholesterol-Binding Antibiotic Nystatin Induces Expression of Macrophage Inflammatory Protein-1 in Macrophages. Biomolecules \& Therapeutics, 21(1), 42-48. https://doi.org/DOI 10.4062/biomolther.2012.082

Baillat, G., Siret, C., Delamarre, E., \& Luis, J. (2008). Early adhesion induces interaction of FAK and Fyn in lipid domains and activates raft-dependent Akt signaling in SW480 colon cancer cells. Biochimica et Biophysica Acta - Molecular Cell Research, 1783(12), 2323-2331. https://doi.org/10.1016/j.bbamcr.2008.08.008 
Barnabas, N., \& Cohen, D. (2013). Phenotypic and Molecular Characterization of MCF10DCIS and SUM Breast Cancer Cell Lines. International Journal of Breast Cancer. https://doi.org/10.1155/2013/872743

Barouch, W., Prasad, K., Greene, L. E., \& Eisenberg, E. (1994). ATPase activity associated with the uncoating of clathrin baskets by Hsp70. Journal of Biological Chemistry, 269(46), 28563-28568.

Bautista, S. J., Boras, I., Vissa, A., Mecica, N., Yip, C. M., Kim, P. K., \& Antonescu, C. N. (2018). mTOR complex 1 controls the nuclear localization and function of glycogen synthase kinase $3 \beta$. Journal of Biological Chemistry. https://doi.org/10.1074/jbc.RA118.002800

Bean, J., Brennan, C., Shih, J.-Y., Riely, G., Viale, A., Wang, L., ... Pao, W. (2007). MET amplification occurs with or without T790M mutations in EGFR mutant lung tumors with acquired resistance to gefitinib or erlotinib. Proceedings of the National Academy of Sciences. https://doi.org/10.1073/pnas.0710370104

Beviglia, L., Matsumoto, K., Lin, C. S., Ziober, B. L., \& Kramer, R. H. (1997). Expression of the cMet/HGF receptor in human breast carcinoma: Correlation with tumor progression. International Journal of Cancer. https://doi.org/10.1002/(SICI)1097-0215(19970620)74:3<301::AIDIJC12>3.0.CO;2-E

Biscardi, J. S., Maa, M.-C., Tice, D. a., Cox, M. E., Leu, T.-H., \& Parsons, S. J. (1999). c-Src-mediated Phosphorylation of the Epidermal Growth Factor Receptor on Tyr845 and Tyr1101 Is Associated with Modulation of Receptor Function. Journal of Biological Chemistry, 274(12), 8335-8343. https://doi.org/10.1074/jbc.274.12.8335

Bonazzi, M., Vasudevan, L., Mallet, A., Sachse, M., Sartori, A., Prevost, M.-C., ... Cossart, P. (2011). Clathrin phosphorylation is required for actin recruitment at sites of bacterial adhesion and internalization. The Journal of Cell Biology, 195(3), 525-536. https://doi.org/10.1083/jcb.201105152

Bone, L. N., Dayam, R. M., Lee, M., Kono, N., Fairn, G. D., Arai, H., ... Antonescu, C. N. (2017). The acyltransferase LYCAT controls specific phosphoinositides and related membrane traffic. Molecular Biology of the Cell, 28(1). https://doi.org/10.1091/mbc.E16-09-0668

Borisov, N., Aksamitiene, E., Kiyatkin, A., Legewie, S., Berkhout, J., Maiwald, T., ... Kholodenko, B. N. (2009). Systems-level interactions between insulin-EGF networks amplify mitogenic signaling. Molecular Systems Biology. https://doi.org/10.1038/msb.2009.19

Brodsky, F. M. (1988). Living with clathrin: Its role in intracellular membrane traffic. Science. https://doi.org/10.1126/science.2904698

Brodsky, F. M. (2012). Diversity of Clathrin Function: New Tricks for an Old Protein. Annual Review of Cell and Developmental Biology, 28(1), 309-336. https://doi.org/10.1146/annurev-cellbio-101011155716

Bromann, P. A., Korkaya, H., \& Courtneidge, S. A. (2004). The interplay between Src family kinases and receptor tyrosine kinases, 7957-7968. https://doi.org/10.1038/sj.onc.1208079 
Brunet, A., Bonni, A., Zigmond, M. J., Lin, M. Z., Juo, P., Hu, L. S., .. Greenberg, M. E. (1999). Akt promotes cell survival by phosphorylating and inhibiting a forkhead transcription factor. Cell. https://doi.org/10.1016/S0092-8674(00)80595-4

Burke, P., Schooler, K., \& Wiley, H. S. (2001). Regulation of Epidermal Growth Factor Receptor Signaling by Endocytosis and Intracellular Trafficking. Molecular Biology of the Cell. https://doi.org/10.1091/mbc.12.6.1897

Burns, C. M., Sakaguchi, K., Appella, E., \& Ashwell, J. D. (1994). CD45 regulation of tyrosine phosphorylation and enzyme activity of src family kinases. Journal of Biological Chemistry.

Busch, D. J., Houser, J. R., Hayden, C. C., Sherman, M. B., Lafer, E. M., \& Stachowiak, J. C. (2015). Intrinsically disordered proteins drive membrane curvature. Nature Communications. https://doi.org/10.1038/ncomms8875

Carpentersllil, G. (1984). Glycosylation of the Epidermal Growth Factor Receptor in A-431 Cells, 259(20), 12586-12594.

Cataldo, V. D., Gibbons, D. L., Pérez-Soler, R., \& Quintás-Cardama, A. (2011). Treatment of NonSmall-Cell Lung Cancer with Erlotinib or Gefitinib. New England Journal of Medicine. https://doi.org/10.1056/nejmct0807960

Cestra, G., Castagnoli, L., Dente, L., Minenkova, O., Petrelli, A., Migone, N., ... Cesareni, G. (1999). The SH3 domains of endophilin and amphiphysin bind to the proline-rich region of synaptojanin 1 at distinct sites that display an unconventional binding specificity. Journal of Biological Chemistry. https://doi.org/10.1074/jbc.274.45.32001

Chan, P.-C., Sudhakar, J. N., Lai, C.-C., \& Chen, H.-C. (2010). Differential phosphorylation of the docking protein Gab1 by c-Src and the hepatocyte growth factor receptor regulates different aspects of cell functions. Oncogene, 29(5), 698-710. https://doi.org/10.1038/onc.2009.363

Chan, W., Sit, S.-T., \& Manser, E. (2011). The Cdc42-associated kinase ACK1 is not autoinhibited but requires Src for activation. The Biochemical Journal, 435, 355-364. https://doi.org/10.1042/BJ20102156

Chan, W., Tian, R., Lee, Y. F., Sit, S. T., Lim, L., \& Manser, E. (2009). Down-regulation of active ACK1 is mediated by association with the E3 ubiquitin ligase Nedd4-2. Journal of Biological Chemistry, 284(12), 8185-8194. https://doi.org/10.1074/jbc.M806877200

Chappie, J. S., Mears, J. A., Fang, S., Leonard, M., Schmid, S. L., Milligan, R. A., ... Dyda, F. (2011). A pseudoatomic model of the dynamin polymer identifies a hydrolysis-dependent powerstroke. Cell. https://doi.org/10.1016/j.cell.2011.09.003

Chen, C. Y., \& Brodsky, F. M. (2005). Huntingtin-interacting protein 1 (Hip1) and Hip1-related protein (Hip1R) bind the conserved sequence of clathrin light chains and thereby influence clathrin assembly in vitro and actin distribution in vivo. Journal of Biological Chemistry. https://doi.org/10.1074/jbc.M408454200

Chen, H., Fre, S., Slepnev, V. I., Capua, M. R., Takei, K., Butler, M. H., ... De Camilli, P. (1998). Epsin is an EH-domain-binding protein implicated in clathrin-mediated endocytosis. Nature, 394(6695), 793-797. https://doi.org/10.1038/29555 
Chen, W. S., Xu, P. Z., Gottlob, K., Chen, M. L., Sokol, K., Shiyanova, T., ... Hay, N. (2001). Growth retardation and increased apoptosis in mice with homozygous disruption of the akt1 gene. Genes and Development. https://doi.org/10.1101/gad.913901

Cheng, J. Q., Ruggeri, B., Klein, W. M., Sonoda, G., Altomare, D. A., Watson, D. K., \& Testa, J. R. (2002). Amplification of AKT2 in human pancreatic cells and inhibition of AKT2 expression and tumorigenicity by antisense RNA. Proceedings of the National Academy of Sciences. https://doi.org/10.1073/pnas.93.8.3636

Cho, H., Thorvaldsen, J. L., Chu, Q., Feng, F., \& Birnbaum, M. J. (2001). Akt1/PKB $\alpha$ Is Required for Normal Growth but Dispensable for Maintenance of Glucose Homeostasis in Mice. Journal of Biological Chemistry. https://doi.org/10.1074/jbc.C100462200

Choudhury, R. (2005). Lowe Syndrome Protein OCRL1 Interacts with Clathrin and Regulates Protein Trafficking between Endosomes and the Trans-Golgi Network. Molecular Biology of the Cell. https://doi.org/10.1091/mbc.e05-02-0120

Cohen, M. H. (2005). FDA Drug Approval Summary: Erlotinib (Tarceva(R)) Tablets. The Oncologist. https://doi.org/10.1634/theoncologist.10-7-461

Cohen, Martin H., Williams, G. A., Sridhara, R., Chen, G., McGuinn, W. D., Morse, D., ... Pazdur, R. (2004). United States Food and Drug Administration Drug Approval Summary: Gefitinib (ZD1839; Iressa) Tablets. Clinical Cancer Research. https://doi.org/10.1158/1078-0432.CCR-03-0564

Cohen, S. (1983). The Epidermal Growth Factor (EGF) Family. Cancer, 51, 1787-1791.

Cohen, S., Ushiro, H., Stoscheck, C., \& Chinkers, M. (1982). A native 170,000 epidermal growth factor receptor-kinase complex from shed plasma membrane vesicles. Journal of Biological Chemistry.

Cohen, Stanley, \& Carpenter, G. (1990). Epidermal Growth Factor. Biochemistry, 265(14), 7709-7712. Retrieved from http://www.springerlink.com/index/122465U384255839.pdf

Collin, G., Franco, M., Simon, V., Bénistant, C., \& Roche, S. (2007). The Tom1L1-clathrin heavy chain complex regulates membrane partitioning of the tyrosine kinase Src required for mitogenic and transforming activities. Molecular and Cellular Biology, 27(21), 7631-7640. https://doi.org/10.1128/MCB.00543-07

Collins, B. M., McCoy, A. J., Kent, H. M., Evans, P. R., \& Owen, D. J. (2002). Molecular architecture and functional model of the endocytic AP2 complex. Cell, 109(4), 523-535. https://doi.org/10.1016/S0092-8674(02)00735-3

Cong, L.-N., Chen, H., Li, Y., Zhou, L., McGibbon, M. A., Taylor, S. I., \& Quon, M. J. (2014). Physiological Role of Akt in Insulin-Stimulated Translocation of GLUT4 in Transfected Rat Adipose Cells. Molecular Endocrinology, 11(13), 1881-1890. https://doi.org/10.1210/mend.11.13.0027

Conner, S. D., \& Schmid, S. L. (2002). Identification of an adaptor-associated kinase, AAK1, as a regulator of clathrin-mediated endocytosis. Journal of Cell Biology, 156(5), 921-929. https://doi.org/10.1083/jcb.200108123 
Conner, S. D., \& Schmid, S. L. (2005). CVAK104 is a novel poly-L-lysine-stimulated kinase that targets the ??2-subunit of AP2. Journal of Biological Chemistry, 280(22), 21539-21544.

https://doi.org/10.1074/jbc.M502462200

Conner, S. D., Schröter, T., \& Schmid, S. L. (2003). AAK1-mediated $\mu 2$ phosphorylation is stimulated by assembled clathrin. Traffic. https://doi.org/10.1046/j.1398-9219.2003.0142.x

Cooke, M. P., Abraham, K. M., Forbush, K. A., \& Perimutter, R. M. (1991). Regulation of T cell receptor signaling by a src family protein-tyrosine kinase (p59fyn). Cell. https://doi.org/10.1016/00928674(91)90162-R

Cross, D. A. E., Alessi, D. R., Cohen, P., Andjelkovich, M., \& Hemmings, B. A. (1995). Inhibition of glycogen synthase kinase-3 by insulin mediated by protein kinase B. Nature. https://doi.org/10.1038/378785a0

Crotzer, V. L., Mabardy, A. S., Weiss, A., \& Brodsky, F. M. (2004). T Cell Receptor Engagement Leads to Phosphorylation of Clathrin Heavy Chain during Receptor Internalization. The Journal of Experimental Medicine, 199(7), 981-991. https://doi.org/10.1084/jem.20031105

Crupi, M. J. F., Richardson, D. S., \& Mulligan, L. M. (2014). Abstract 3450: Internalization and recycling of RET receptor tyrosine kinase isoforms. https://doi.org/10.1158/1538-7445.am2014-3450

Cruz, J., Reis-Filho, J. S., Silva, P., \& Lopes, J. M. (2003). Expression of c-met tyrosine kinase receptor is biologically and prognostically relevant for primary cutaneous malignant melanomas. Oncology. https://doi.org/10.1159/000071207

Dannhauser, P. N., Camus, S. M., Sakamoto, K., Sadacca, L. A., Torres, J. A., Camus, M. D., ... Brodsky, F. M. (2017). CHC22 and CHC17 clathrins have distinct biochemical properties and display differential regulation and function. Journal of Biological Chemistry. https://doi.org/10.1074/jbc.M117.816256

De Angelis, D. A. (2004). Fluorescence Resonance Energy Transfer Fret. In Encyclopedia of Medical Genomics and Proteomics. https://doi.org/10.3109/9780203997352.101

De Heuvel, E., Bell, A. W., Ramjaun, A. R., Wong, K., Sossin, W. S., \& McPherson, P. S. (1997). Identification of the major synaptojanin-binding proteins in brain. Journal of Biological Chemistry. https://doi.org/10.1074/jbc.272.13.8710

de Melker, A. A., van der Horst, G., Calafat, J., Jansen, H., \& Borst, J. (2001). c-Cbl ubiquitinates the EGF receptor at the plasma membrane and remains receptor associated throughout the endocytic route. Journal of Cell Science.

deGraffenried, L. A., Fulcher, L., Friedrichs, W. E., Grünwald, V., Ray, R. B., \& Hidalgo, M. (2004). Reduced PTEN expression in breast cancer cells confers susceptibility to inhibitors of the PI3 kinase/Akt pathway. Annals of Oncology, 15(10), 1510-1516. https://doi.org/10.1093/annonc/mdh388

Dell'Angelica, E. C. (2001). Clathrin-binding proteins: Got a motif? Join the network! Trends in Cell Biology, 11(8), 315-318. https://doi.org/10.1016/S0962-8924(01)02043-8 
Dell'Angelica, Esteban C., Klumperman, J., Stoorvogel, W., \& Bonifacino, J. S. (1998). Association of the AP-3 adaptor complex with clathrin. Science. https://doi.org/10.1126/science.280.5362.431

Delos Santos, R., Bautista, S., Bone, L., Lucarelli, S., Dayam, R., Botelho, R., \& Antonescu, C. (2017). Selective control of clathrin- mediated endocytosis and clathrin-dependent signaling by phospholipase C and Ca2+ signals. Molecular Biology of the Cell, 28, 2802-2818

Delos Santos, R. C., Garay, C., \& Antonescu, C. N. (2015). Charming neighborhoods on the cell surface: Plasma membrane microdomains regulate receptor tyrosine kinase signaling. Cellular Signalling, 27(10), 1963-1976. https://doi.org/10.1016/j.cellsig.2015.07.004

Di Fiore, P. P., Pierce, J. H., Kraus, M. H., Segatto, O., King, C. R., \& Aaronson, S. A. (1987). ErbB-2 is a potent oncogene when overexpressed in NIH/3T3 cells. Science. https://doi.org/10.1126/science.2885917

Di Guglielmo, G. M., Baass, P. C., Ou, W. J., Posner, B. I., \& Bergeron, J. J. (1994). Compartmentalization of SHC, GRB2 and mSOS, and hyperphosphorylation of Raf-1 by EGF but not insulin in liver parenchyma. The EMBO Journal. https://doi.org/10.1002/j.14602075.1994.tb06747.x

Ducker, C. E. (2005). Two N-Myristoyltransferase Isozymes Play Unique Roles in Protein Myristoylation, Proliferation, and Apoptosis. Molecular Cancer Research. https://doi.org/10.1158/1541-7786.mcr-05-0037

Ebner, M., Lučić, I., Leonard, T. A., \& Yudushkin, I. (2017). PI(3,4,5)P3 Engagement Restricts Akt Activity to Cellular Membranes. Molecular Cell, 65(3), 416-431.e6. https://doi.org/10.1016/j.molcel.2016.12.028

Eden, E. R., White, I. J., Tsapara, A., \& Futter, C. E. (2010). Membrane contacts between endosomes and ER provide sites for PTP1B-epidermal growth factor receptor interaction. Nature Cell Biology. https://doi.org/10.1038/ncb2026

Ehrlich, M., Boll, W., Van Oijen, A., Hariharan, R., Chandran, K., Nibert, M. L., \& Kirchhausen, T. (2004). Endocytosis by random initiation and stabilization of clathrin-coated pits. Cell. https://doi.org/10.1016/j.cell.2004.08.017

Eichel, K., Jullié, D., \& von Zastrow, M. (2016). $\beta$-Arrestin drives MAP kinase signalling from clathrincoated structures after GPCR dissociation. Nature Cell Biology, 18(3). https://doi.org/10.1038/ncb3307

Engelman, J. A., Zejnullahu, K., Mitsudomi, T., Song, Y., Hyland, C., Joon, O. P., ... Jänne, P. A. (2007). MET amplification leads to gefitinib resistance in lung cancer by activating ERBB3 signaling. Science. https://doi.org/10.1126/science.1141478

Espina, V., \& Liotta, L. A. (2011). What is the malignant nature of human ductal carcinoma in situ? Nature Reviews Cancer. https://doi.org/10.1038/nrc2950

Filipp, D., \& Julius, M. (2004). Lipid rafts: resolution of the "fyn problem"? Molecular Immunology, 41(6-7), 645-656. https://doi.org/10.1016/j.molimm.2004.04.011 
Filipp, D., Zhang, J., Leung, B. L., Shaw, A., Levin, S. D., Veillette, A., \& Julius, M. (2003). Regulation of Fyn through translocation of activated Lck into lipid rafts. The Journal of Experimental Medicine, 197(9), 1221-1227. https://doi.org/10.1084/jem.20022112

FISHER, D. A., \& LAKSHMANAN, J. (1990). Metabolism and Effects of Epidermal Growth Factor and Related Growth Factors in Mammals*. Endocrine Reviews, 11(3), 418-442. https://doi.org/10.1210/edrv-11-3-418

Fletterick, R. J., Liu, S. H., Hwang, P. K., Chen, L., Lin, K., Earnest, T. N., ... Brodsky, F. M. (1999). Clathrin self-assembly is mediated by a tandemly repeated superhelix. Nature, 399(6734), 371-375. https://doi.org/10360576

Ford, M. G., Mills, I. G., Peter, B. J., Vallis, Y., Praefcke, G. J., Evans, P. R., \& McMahon, H. T. (2002). Curvature of clathrin-coated pits driven by epsin. Nature, 419(6905), 361-366.

Fotin, A., Cheng, Y., Grigorieff, N., Walz, T., Harrison, S. C., \& Kirchhausen, T. (2004). Structure of an auxilin-bound clathrin coat and its implications for the mechanism of uncoating. Nature. https://doi.org/10.1038/nature03078

Fouladkou, F., Lu, C., Jiang, C., Zhou, L., She, Y., Walls, J. R., ... Rotin, D. (2010). The ubiquitin ligase Nedd4-1 is required for heart development and is a suppressor of thrombospondin-1. Journal of Biological Chemistry. https://doi.org/10.1074/jbc.M109.082347

Freed, D. M., Bessman, N. J., Kiyatkin, A., Salazar-Cavazos, E., Byrne, P. O., Moore, J. O., ... Lemmon, M. A. (2017). EGFR Ligands Differentially Stabilize Receptor Dimers to Specify Signaling Kinetics. Cell, 171(3), 683-695.e18. https://doi.org/10.1016/j.cell.2017.09.017

Fu, Y.-N., Yeh, C.-L., Cheng, H. H. Y., Yang, C.-H., Tsai, S.-F., Huang, S.-F., \& Chen, Y.-R. (2008). EGFR mutants found in non-small cell lung cancer show different levels of sensitivity to suppression of Src: implications in targeting therapy. Oncogene, 27(7), 957-965. https://doi.org/10.1038/sj.onc.1210684

Funderburk, S. F., Wang, Q. J., \& Yue, Z. (2010). The Beclin 1-VPS34 complex - at the crossroads of autophagy and beyond. Trends in Cell Biology. https://doi.org/10.1016/j.tcb.2010.03.002

Galisteo, M., \& Yang, Y. (2006). Activation of the nonreceptor protein tyrosine kinase Ack by multiple extracellular stimuli. Proceedings of the ..., 103(26), 9796-9801. Retrieved from http://www.pnas.org/content/103/26/9796.short

Gallop, J. L., Jao, C. C., Kent, H. M., Butler, P. J. G., Evans, P. R., Langen, R., \& McMahon, H. T. (2006). Mechanism of endophilin N-BAR domain-mediated membrane curvature. EMBO Journal. https://doi.org/10.1038/sj.emboj.7601174

Gao, T., Furnari, F., \& Newton, A. C. (2005). PHLPP: A phosphatase that directly dephosphorylates Akt, promotes apoptosis, and suppresses tumor growth. Molecular Cell. https://doi.org/10.1016/j.molcel.2005.03.008

Garay, C., Judge, G., Lucarelli, S., Bautista, S., Pandey, R., Singh, T., \& Antonescu, C. N. (2015). Epidermal growth factor-stimulated Akt phosphorylation requires clathrin or ErbB2 but not receptor endocytosis. Molecular Biology of the Cell, 26(19). https://doi.org/10.1091/mbc.E14-09-1412 
Garay, Camilo, Judge, G., Lucarelli, S., Bautista, S., Pandey, R., Singh, T., \& Antonescu, C. N. C. N. (2015). Epidermal growth factor-stimulated Akt phosphorylation requires clathrin or ErbB2 but not receptor endocytosis. Molecular Biology of the Cell, 26(19), 3504-3519. https://doi.org/10.1091/mbc.E14-09-1412

Garofalo, R. S., Orena, S. J., Rafidi, K., Torchia, A. J., Stock, J. L., Hildebrandt, A. L., ... Coleman, K. G. (2003). Severe diabetes, age-dependent loss of adipose tissue, and mild growth deficiency in mice lacking Akt2/PKBß. Journal of Clinical Investigation. https://doi.org/10.1172/JCI16885

Githaka, J. M., Vega, A. R., Baird, M. A., Davidson, M. W., Jaqaman, K., \& Touret, N. (2016). Ligandinduced growth and compaction of CD36 nanoclusters enriched in Fyn induces Fyn signaling. Journal of Cell Science, 129(22), 4175-4189. https://doi.org/10.1242/jcs.188946

Gnoni, A., Marech, I., Silvestris, N., Vacca, A., \& Lorusso, V. (2011). Dasatinib: An Anti-Tumour Agent via Src Inhibition. Current Drug Targets, 12(4), 563-578. https://doi.org/10.2174/138945011794751591

Gocek, E., Moulas, A. N., \& Studzinski, G. P. (2014). Non-receptor protein tyrosine kinases signaling pathways in normal and cancer cells. Critical Reviews in Clinical Laboratory Sciences, 51(3), 125137. https://doi.org/10.3109/10408363.2013.874403

Goh, L. K., Huang, F., Kim, W., Gygi, S., \& Sorkin, A. (2010). Multiple mechanisms collectively regulate clathrin-mediated endocytosis of the epidermal growth factor receptor. The Journal of Cell Biology, 189(5), 871-883. https://doi.org/10.1083/jcb.201001008

Gonzalez, E., McGraw, T. E. (2009). The Akt kinases: Isoform specificity in metabolism and cancer. Cell Cycle, 8(16), 2502-2508.

Gonzalez, E., \& McGraw, T. E. (2009). The Akt kinases: Isoform specificity in metabolism and cancer. Cell Cycle, 8(16), 2502-2508. https://doi.org/10.4161/cc.8.16.9335

Gottlieb-Abraham, E., Gutman, O., Pai, G. M., Rubio, I., \& Henis, Y. I. (2016). The residue at position 5 of the N-terminal region of Src and Fyn modulates their myristoylation, palmitoylation, and membrane interactions. Molecular Biology of the Cell. https://doi.org/10.1091/mbc.e16-08-0622

Goulden, B. D., Pacheco, J., Dull, A., Zewe, J. P., Deiters, A., \& Hammond, G. R. V. (2019). A highavidity biosensor reveals plasma membrane $\mathrm{PI}(3,4) \mathrm{P} 2$ is predominantly a class I PI3K signaling product. Journal of Cell Biology. https://doi.org/10.1083/jcb.201809026

Grossier, J. P., Xouri, G., Goud, B., \& Schauer, K. (2014). Cell adhesion defines the topology of endocytosis and signaling. EMBO Journal. https://doi.org/10.1002/embj.201385284

Grøvdal, Lene Melsaether, Johannessen, L. E., Rødland, M. S., Madshus, I. H., \& Stang, E. (2008). Dysregulation of Ack1 inhibits down-regulation of the EGF receptor. Experimental Cell Research, 314(6), 1292-1300. https://doi.org/10.1016/j.yexcr.2007.12.017

Grøvdal, Lene Melsæther, Stang, E., Sorkin, A., \& Madshus, I. H. (2004). Direct interaction of Cbl with pTyr 1045 of the EGF receptor (EGFR) is required to sort the EGFR to lysosomes for degradation. Experimental Cell Research. https://doi.org/10.1016/j.yexcr.2004.07.003 
Grove, J., Metcalf, D. J., Knight, A. E., Wavre-Shapton, S. T., Sun, T., Protonotarios, E. D., ... Marsh, M. (2014). Flat clathrin lattices: stable features of the plasma membrane. Molecular Biology of the Cell, 25(22), 3581-3594. https://doi.org/10.1091/mbc.e14-06-1154

Gryaznova, T., Kropyvko, S., Burdyniuk, M., Gubar, O., Kryklyva, V., Tsyba, L., \& Rynditch, A. (2015). Intersectin adaptor proteins are associated with actin-regulating protein WIP in invadopodia. Cellular Signalling. https://doi.org/10.1016/j.cellsig.2015.03.006

Gu, M., Liu, Q., Watanabe, S., Sun, L., Hollopeter, G., Grant, B. D., \& Jorgensen, E. M. (2013). AP2 hemicomplexes contribute independently to synaptic vesicle endocytosis. ELife. https://doi.org/10.7554/eLife.00190

Guo, S., Stolz, L. E., Lemrow, S. M., \& York, J. D. (1999). SAC1-like domains of yeast SAC1, INP52, and INP53 and of human synaptojanin encode polyphosphoinositide phosphatases. Journal of Biological Chemistry. https://doi.org/10.1074/jbc.274.19.12990

Gustafsson, M. G. L. (2000). Surpassing the lateral resolution limit by a factor of two using structured illumination microscopy. Journal of Microscopy. https://doi.org/10.1046/j.1365-2818.2000.00710.x

Hammond, D. E., Urbé, S., Vande Woude, G. F., \& Clague, M. J. (2001). Down-regulation of MET, the receptor for hepatocyte growth factor. Oncogene. https://doi.org/10.1038/sj.onc.1204475

Harari, D., \& Yarden, Y. (2000). Molecular mechanisms underlying ErbB2/HER2 action in breast cancer. Oncogene. https://doi.org/10.1038/sj.onc.1203973

Haugh, J. M., Huang, A. C., Wiley, H. S., Wells, A., \& Lauffenburger, D. A. (1999). Internalized epidermal growth factor receptors participate in the activation of p21(ras) in fibroblasts. Journal of Biological Chemistry. https://doi.org/10.1074/jbc.274.48.34350

Heasman, S. J., \& Ridley, A. J. (2008). Mammalian Rho GTPases: New insights into their functions from in vivo studies. Nature Reviews Molecular Cell Biology. https://doi.org/10.1038/nrm2476

Henne, W. M., Boucrot, E., Meinecke, M., Evergren, E., Vallis, Y., Mittal, R., \& McMahon, H. T. (2010). FCHo proteins are nucleators of clathrin-mediated endocytosis. Science (New York, N.Y.), 328(5983), 1281-1284. https://doi.org/10.1126/science.1188462

Herbst, R. S., Heymach, J. V., \& Lippman, S. M. (2008). Lung cancer Lung cancer Lung cancer, 359(November), 1367-1380.

Hicke, L., \& Dunn, R. (2003). Regulation of Membrane Protein Transport by Ubiquitin and UbiquitinBinding Proteins. Annual Review of Cell and Developmental Biology. https://doi.org/10.1146/annurev.cellbio.19.110701.154617

Holgado-Madruga, M., Emlet, D. R., Moscatello, D. K., Godwin, A. K., \& Wong, A. J. (1996). A Grb2associated docking protein in EGF- and insulin-receptor signalling. Nature, 379(6565), 560-564. https://doi.org/10.1038/379560a0

Horvath, C. a J., Vanden Broeck, D., Boulet, G. a V, Bogers, J., \& De Wolf, M. J. S. (2007). Epsin: Inducing membrane curvature. International Journal of Biochemistry and Cell Biology, 39(10), 1765-1770. https://doi.org/10.1016/j.biocel.2006.12.004 
Howlin, J., Rosenkvist, J., \& Andersson, T. (2008). TNK2 preserves epidermal growth factor receptor expression on the cell surface and enhances migration and invasion of human breast cancer cells. Breast Cancer Research : BCR, 10(2), R36. https://doi.org/10.1186/bcr2087

Huang, R., Fang, P., Hao, Z., \& Kay, B. K. (2016). Directed evolution of a highly specific FN3 monobody to the SH3 domain of human lyn tyrosine kinase. PLOS ONE, 11(1), 1-22. https://doi.org/10.1371/journal.pone.0145872

Hughes, A. L. (1996). Evolution of thesrc-related protein tyrosine kinases. Journal of Molecular Evolution, 42(2), 247-256. https://doi.org/10.1007/BF02198851

Huh, C.-G., Factor, V. M., Sanchez, A., Uchida, K., Conner, E. A., \& Thorgeirsson, S. S. (2004). Hepatocyte growth factor/c-met signaling pathway is required for efficient liver regeneration and repair. Proceedings of the National Academy of Sciences. https://doi.org/10.1073/pnas.0306068101

Hynes, N. E., \& MacDonald, G. (2009). ErbB receptors and signaling pathways in cancer. Current Opinion in Cell Biology, 21(2), 177-184. https://doi.org/10.1016/j.ceb.2008.12.010

Ibach, J., Radon, Y., Gelléri, M., Sonntag, M. H., Brunsveld, L., Bastiaens, P. I. H., \& Verveer, P. J. (2015). Single Particle Tracking Reveals that EGFR Signaling Activity Is Amplified in ClathrinCoated Pits. Plos One, 10(11), e0143162. https://doi.org/10.1371/journal.pone.0143162

Ilangumaran, S., Arni, S., van Echten-Deckert, G., Borisch, B., \& Hoessli, D. C. (1999). Microdomaindependent regulation of Lck and Fyn protein-tyrosine kinases in T lymphocyte plasma membranes. Molecular Biology of the Cell, 10(4), 891-905.

Imae, R., Inoue, T., Nakasaki, Y., Uchida, Y., Ohba, Y., Kono, N., ... Arai, H. (2012). LYCAT, a homologue of C. elegans acl-8, acl-9, and acl-10, determines the fatty acid composition of phosphatidylinositol in mice. The Journal of Lipid Research, 53(3), 335-347. https://doi.org/10.1194/jlr.M018655

Inoki, K., Li, Y., Zhu, T., Wu, J., \& Guan, K. L. (2002). TSC2 is phosphorylated and inhibited by Akt and suppresses mTOR signalling. Nature Cell Biology. https://doi.org/10.1038/ncb839

Iqbal, K., Liu, F., Gong, C.-X., \& Grundke-Iqbal, I. (2010). Tau in Alzheimer Disease and Related Tauopathies. Current Alzheimer Research. https://doi.org/10.2174/156720510793611592

Jaqaman, K., Loerke, D., Mettlen, M., Kuwata, H., Grinstein, S., Schmid, S. L., \& Danuser, G. (2008). Robust single-particle tracking in live-cell time-lapse sequences. Nature Methods, 5(8), 695-702. https://doi.org/10.1038/nmeth.1237

Johannessen, L. E., Pedersen, N. M., Pedersen, K. W., Madshus, I. H., \& Stang, E. (2005). Activation of the Epidermal Growth Factor (EGF) Receptor Induces Formation of EGF Receptor- and Grb2Containing Clathrin-Coated Pits. Molecular and Cellular Biology. https://doi.org/10.1128/mcb.26.2.389-401.2006

Jura, N., Endres, N. F., Engel, K., Deindl, S., Das, R., Lamers, M. H., ... Kuriyan, J. (2009). Mechanism for Activation of the EGF Receptor Catalytic Domain by the Juxtamembrane Segment. Cell. https://doi.org/10.1016/j.cell.2009.04.025 
Kadlecova, Z., Spielman, S. J., Loerke, D., Mohanakrishnan, A., Reed, D. K., \& Schmid, S. L. (2017). Regulation of clathrin-mediated endocytosis by hierarchical allosteric activation of AP2. The Journal of Cell Biology, 216(1), 167-179. https://doi.org/10.1083/jcb.201608071

Kaidanovich-Beilin, O., \& Woodgett, J. R. (2011). GSK-3: Functional Insights from Cell Biology and Animal Models. Frontiers in Molecular Neuroscience. https://doi.org/10.3389/fnmol.2011.00040

Kaksonen, M., \& Roux, A. (2018). Mechanisms of clathrin-mediated endocytosis. Nature Reviews Molecular Cell Biology. https://doi.org/10.1038/nrm.2017.132

Kassenbrock, C. K., Hunter, S., Garl, P., Johnson, G. L., \& Anderson, S. M. (2002). Inhibition of Src Family Kinases Blocks Epidermal Growth Factor (EGF)-induced Activation of Akt, Phosphorylation of c-Cbl, and Ubiquitination of the EGF Receptor. The Journal of Biological Chemistry, 277(28), 24967-24975. https://doi.org/10.1074/jbc.M201026200

Kaszuba, K., Grzybek, M., Orłowski, A., Danne, R., Róg, T., Simons, K., ... Vattulainen, I. (2015). N Glycosylation as determinant of epidermal growth factor receptor conformation in membranes . Proceedings of the National Academy of Sciences. https://doi.org/10.1073/pnas.1503262112

Katoh, Y., Imakagura, H., Futatsumori, M., \& Nakayama, K. (2006). Recruitment of clathrin onto endosomes by the Tom1-Tollip complex. Biochemical and Biophysical Research Communications. https://doi.org/10.1016/j.bbrc.2005.12.156

Kaufman, A. C., Salazar, S. V., Haas, L. T., Yang, J., Kostylev, M. A., Jeng, A. T., ... Strittmatter, S. M. (2015). Fyn inhibition rescues established memory and synapse loss in Alzheimer mice. Annals of Neurology. https://doi.org/10.1002/ana.24394

Kerscher, O., Felberbaum, R., \& Hochstrasser, M. (2006). Modification of Proteins by Ubiquitin and Ubiquitin-Like Proteins. Annual Review of Cell and Developmental Biology. https://doi.org/10.1146/annurev.cellbio.22.010605.093503

Kirchhausen, Tom, Owen, D., \& Harrison, S. C. (2014). Molecular structure, function, and dynamics of clathrin-mediated membrane traffic. Cold Spring Harbor Perspectives in Biology, 6(5), a016725. https://doi.org/10.1101/cshperspect.a016725

Kirchhausen, Tomas, Bonifacino, J. S., \& Riezman, H. (1997). Linking cargo to vesicle formation: Receptor tail interactions with coat proteins. Current Opinion in Cell Biology. https://doi.org/10.1016/S0955-0674(97)80024-5

Kirchhausen, Tomas, \& Harrison, S. C. (1981). Protein organization in clathrin trimers. Cell, 23(3), 755761. https://doi.org/10.1016/0092-8674(81)90439-6

Kiyatkin, A., Aksamitiene, E., Markevich, N. I., Borisov, N. M., Hoek, J. B., \& Kholodenko, B. N. (2006). Scaffolding protein Grb2-associated binder 1 sustains epidermal growth factor-induced mitogenic and survival signaling by multiple positive feedback loops. The Journal of Biological Chemistry, 281(29), 19925-19938. https://doi.org/10.1074/jbc.M600482200

Koegl, M., Zlatkine, P., Ley, S. C., Courtneidge, S. A., \& Magee, A. I. (1994). Palmitoylation of multiple Src-family kinases at a homologous N-terminal motif. The Biochemical Journal. 
Kozlov, M. M. (1999). Dynamin: Possible mechanism of "Pinchase" action. Biophysical Journal. https://doi.org/10.1016/S0006-3495(99)76917-1

Kural, C., Tacheva-Grigorova, S. K., Boulant, S., Cocucci, E., Baust, T., Duarte, D., \& Kirchhausen, T. (2012). Dynamics of Intracellular Clathrin/AP1- and Clathrin/AP3-Containing Carriers. Cell Reports. https://doi.org/10.1016/j.celrep.2012.09.025

Le Clainche, C., Pauly, B. S., Zhang, C. X., Engqvist-Goldstein, Å. E. Y., Cunningham, K., \& Drubin, D. G. (2007). A Hip1R-cortactin complex negatively regulates actin assembly associated with endocytosis. EMBO Journal. https://doi.org/10.1038/sj.emboj.7601576

Lee, Y. R., Chen, M., \& Pandolfi, P. P. (2018). The functions and regulation of the PTEN tumour suppressor: new modes and prospects. Nature Reviews Molecular Cell Biology. https://doi.org/10.1038/s41580-018-0015-0

Legendre-Guillemin, V., Metzler, M., Lemaire, J. F., Philie, J., Gan, L., Hayden, M. R., \& McPherson, P. S. (2005). Huntingtin Interacting Protein 1 (HIP1) regulates clathrin assembly through direct binding to the regulatory region of the clathrin light chain. Journal of Biological Chemistry. https://doi.org/10.1074/jbc.M408430200

Lemmon, M. A. (2008). Membrane recognition by phospholipid-binding domains. Nature Reviews Molecular Cell Biology. https://doi.org/10.1038/nrm2328

Lemmon, M. a, \& Schlessinger, J. (2010). Cell signaling by receptor tyrosine kinases. Cell, 141(7), 11171134. https://doi.org/10.1016/j.cell.2010.06.011

Lengyel, E., Prechtel, D., Resau, J. H., Gauger, K., Welk, A., Lindemann, K., ... Harbeck, N. (2005). cMet overexpression in node-positive breast cancer identifies patients with poor clinical outcome independent of Her2/neu. International Journal of Cancer. https://doi.org/10.1002/ijc.20598

Levkowitz, G., Waterman, H., Ettenberg, S. A., Katz, M., Tsygankov, A. Y., Alroy, I., ... Yarden, Y. (1999). Ubiquitin Ligase Activity and Tyrosine Phosphorylation Underlie Suppression of Growth Factor Signaling by c-Cb1/Sli-1. Molecular Cell, 4(6), 1029-1040. https://doi.org/10.1016/S10972765(00)80231-2

Levkowitz, G., Waterman, H., Zamir, E., Kam, Z., Oved, S., Langdon, W. Y., ... Yarden, Y. (1998). c$\mathrm{Cb} 1 /$ Sli-1 regulates endocytic sorting and ubiquitination of the epidermal growth factor receptor. Genes and Development. https://doi.org/10.1101/gad.12.23.3663

Leyton-Puig, D., Isogai, T., Argenzio, E., van den Broek, B., Klarenbeek, J., Janssen, H., ... Innocenti, M. (2017). Flat clathrin lattices are dynamic actin-controlled hubs for clathrin-mediated endocytosis and signalling of specific receptors. Nature Communications, 8(May), 16068. https://doi.org/10.1038/ncomms 16068

Li, H., Stokes, W., Chater, E., Roy, R., De Bruin, E., Hu, Y., ... Pardo, O. E. (2016). Decreased glutathione biosynthesis contributes to EGFR T790M-driven erlotinib resistance in non-small cell lung cancer. Cell Discovery. https://doi.org/10.1038/celldisc.2016.31

Li, N., Lorinczi, M., Ireton, K., \& Elferink, L. A. (2007). Specific Grb2-mediated interactions regulate clathrin-dependent endocytosis of the cMet-tyrosine kinase. Journal of Biological Chemistry. https://doi.org/10.1074/jbc.M610835200 
Li, S., Schmitz, K. R., Jeffrey, P. D., Wiltzius, J. J. W., Kussie, P., \& Ferguson, K. M. (2005). Structural basis for inhibition of the epidermal growth factor receptor by cetuximab. Cancer Cell. https://doi.org/10.1016/j.ccr.2005.03.003

Li, W., Marshall, C., Mei, L., Dzubow, L., Schmults, C., Dans, M., \& Seykora, J. (2005). Srcasm modulates EGF and Src-kinase signaling in keratinocytes. The Journal of Biological Chemistry, 280(7), 6036-6046. https://doi.org/10.1074/jbc.M406546200

Liang, L., Shen, H., De Camilli, P., \& Duncan, J. (2010). Tracking Clathrin Coated Pits with a Multiple Hypothesis Based Method. Springer. Retrieved from https://link.springer.com/content/pdf/10.1007\%2F978-3-642-15745-5.pdf

Liang, X., Nazarian, A., Erdjument-Bromage, H., Bornmann, W., Tempst, P., \& Resh, M. D. (2001). Heterogeneous Fatty Acylation of Src Family Kinases with Polyunsaturated Fatty Acids Regulates Raft Localization and Signal Transduction. Journal of Biological Chemistry, 276(33), 30987-30994. https://doi.org/10.1074/jbc.M104018200

Lin, Q., Wang, J., Childress, C., \& Yang, W. (2012). The activation mechanism of ACK1 (activated Cdc42-associated tyrosine kinase 1). The Biochemical Journal, 445(2), 255-264. https://doi.org/10.1042/BJ20111575

Liu, A. P., Aguet, F., Danuser, G., \& Schmid, S. L. (2010). Local clustering of transferrin receptors promotes clathrin-coated pit initiation. The Journal of Cell Biology, 191(7), 1381-1393. https://doi.org/10.1083/jcb.201008117

Liu, N. S., Loo, L. S., Loh, E., Seet, L.-F., \& Hong, W. (2009). Participation of Tom1L1 in EGFstimulated endocytosis of EGF receptor. The EMBO Journal, 28(22), 3485-3499. https://doi.org/10.1038/emboj.2009.282

Liu, S. L., Wang, Z. G., Hu, Y., Xin, Y., Singaram, I., Gorai, S., ... Cho, W. (2018). Quantitative Lipid Imaging Reveals a New Signaling Function of Phosphatidylinositol-3,4-Bisphophate: Isoform- and Site-Specific Activation of Akt. Molecular Cell. https://doi.org/10.1016/j.molcel.2018.07.035

Lock, L S, Royal, I., Naujokas, M. A., \& Park, M. (2000). Identification of an atypical Grb2 carboxylterminal SH3 domain binding site in Gab docking proteins reveals Grb2-dependent and independent recruitment of Gab1 to receptor tyrosine kinases. The Journal of Biological Chemistry, 275(40), 31536-31545. https://doi.org/10.1074/jbc.M003597200

Lock, Lisa S., Frigault, M. M., Saucier, C., \& Park, M. (2003). Grb2-independent recruitment of Gab1 requires the $\mathrm{C}$-terminal lobe and structural integrity of the met receptor kinase domain. Journal of Biological Chemistry. https://doi.org/10.1074/jbc.M302675200

Lock, Lisa S., Royal, I., Naujokas, M. A., \& Park, M. (2000). Identification of an atypical Grb2 carboxylterminal SH3 domain binding site in Gab docking proteins reveals Grb2-dependent and independent recruitment of Gab1 to receptor tyorosine kinases. Journal of Biological Chemistry, 275(40), 31536-31545. https://doi.org/10.1074/jbc.M003597200

Loerke, D, Mettlen, M., Yarar, D., Jaqaman, K., Jaqaman, H., Danuser, G., \& Schmid, S. L. (2009). Cargo and dynamin regulate clathrin-coated pit maturation. PLoS Biol, 7(3), e57. 
Loerke, Dinah, Mettlen, M., Schmid, S. L., \& Danuser, G. (2011). Measuring the hierarchy of molecular events during clathrin-mediated endocytosis. Traffic (Copenhagen, Denmark). https://doi.org/10.1111/j.1600-0854.2011.01197.x

Loerke, Dinah, Mettlen, M., Yarar, D., Jaqaman, K., Jaqaman, H., Danuser, G., \& Schmid, S. L. (2009). Cargo and Dynamin Regulate Clathrin-Coated Pit Maturation. PLoS Biology, 7(3), e57. https://doi.org/10.1371/journal.pbio.1000057?

Lombardo, L. J., Lee, F. Y., Chen, P., Norris, D., Barrish, J. C., Behnia, K., ... Borzilleri, R. M. (2004). Discovery of N - ( 2-Chloro-6-methyl- phenyl ) -2- ( 6- ( 4- ( 2-hydroxyethyl ) - (BMS-354825), a Dual Src / Abl Kinase Inhibitor with Potent Antitumor Activity in Preclinical Assays, 6658-6661.

Long, X., Lin, Y., Ortiz-Vega, S., Yonezawa, K., \& Avruch, J. (2005). Rheb binds and regulates the mTOR kinase. Current Biology. https://doi.org/10.1016/j.cub.2005.02.053

Lu, K. V, Zhu, S., Cvrljevic, A., Huang, T. T., Sarkaria, S., Ahkavan, D., ... Mischel, P. S. (2009). Fyn and SRC are effectors of oncogenic epidermal growth factor receptor signaling in glioblastoma patients. Cancer Research, 69(17), 6889-6898. https://doi.org/10.1158/0008-5472.CAN-09-0347

Lucarelli, S., Delos Santos, R. C., \& Antonescu, C. N. (2017). Measurement of epidermal growth factor receptor-derived signals within plasma membrane clathrin structures. Methods in Molecular Biology (Vol. 1652). https://doi.org/10.1007/978-1-4939-7219-7_15

Mahajan, K., \& Mahajan, N. P. (2014). ACK1/TNK2 tyrosine kinase: molecular signaling and evolving role in cancers. Oncogene, 6(August), 1-6. https://doi.org/10.1038/onc.2014.350

Manning, B. D., \& Toker, A. (2017). AKT/PKB Signaling: Navigating the Network. Cell, 169(3), 381405. https://doi.org/10.1016/j.cell.2017.04.001

Manser, E., Leung, T., Salihuddin, H., Tan, L., \& Lim, L. (1993). A non-receptor tyrosine kinase that inhibits the GTPase activity of p21cdc42. Nature, 363(6427), 364-367. https://doi.org/10.1038/363364a0

Marchese, A., Paing, M. M., Temple, B. R. S., \& Trejo, J. (2007). G Protein-Coupled Receptor Sorting to Endosomes and Lysosomes. Annual Review of Pharmacology and Toxicology. https://doi.org/10.1146/annurev.pharmtox.48.113006.094646

Marcotte, R., Zhou, L., Kim, H., Roskelly, C. D., \& Muller, W. J. (2009). c-Src associates with ErbB2 through an interaction between catalytic domains and confers enhanced transforming potential. Molecular and Cellular Biology, 29(21), 5858-5871. https://doi.org/10.1128/MCB.01731-08

Martin, N. P., Mohney, R. P., Dunn, S., Das, M., Scappini, E., \& Bryan, J. P. O. (2006). Intersectin Regulates Epidermal Growth Factor Receptor. Molecular Pharmacology, 70, 1643-1653. https://doi.org/10.1124/mol.106.028274.

Martins, A. S., Ordóñez, J. L., Amaral, A. T., Prins, F., Floris, G., Debiec-Rychter, M., ... de Alava, E. (2011). IGF1R signaling in Ewing Sarcoma is shaped by clathrin-/caveolin-dependent endocytosis. PLoS ONE. https://doi.org/10.1371/journal.pone.0019846

Mattoon, D. R., Lamothe, B., Lax, I., \& Schlessinger, J. (2004a). EGF-stimulated activation of the PI-3K / Akt cell survival pathway, 12, 1-12. https://doi.org/10.1186/1741-7007-2-24 
Mattoon, D. R., Lamothe, B., Lax, I., \& Schlessinger, J. (2004b). The docking protein Gab1 is the primary mediator of EGF-stimulated activation of the PI-3K/Akt cell survival pathway. BMC Biology, 2(1), 24. https://doi.org/10.1186/1741-7007-2-24

Maurer, M. E., \& Cooper, J. A. (2006). The adaptor protein Dab2 sorts LDL receptors into coated pits independently of AP-2 and ARH. Journal of Cell Science, 119(20), 4235-4246. https://doi.org/10.1242/jcs.03217

McLachlan, R. W., Kraemer, A., Helwani, F. M., Kovacs, E. M., \& Yap, A. S. (2007). E-Cadherin Adhesion Activates c-Src Signaling at Cell-Cell Contacts. Molecular Biology of the Cell. https://doi.org/10.1091/mbc.e06-12-1154

McMahon, H. T., \& Boucrot, E. (2011). Molecular mechanism and physiological functions of clathrinmediated endocytosis. Nature Reviews. Molecular Cell Biology, 12(8), 517-533. https://doi.org/10.1038/nrm3151

McMahon, H. T., Wigge, P., \& Smith, C. (1997). Clathrin interacts specifically with amphiphysin and is displaced by dynamin. FEBS Letters, 413(2), 319-322. https://doi.org/10.1016/S00145793(97)00928-9

McPherson, P. S., Garcia, E. P., Slepnev, V. I., David, C., Zhang, X., Grabs, D., .. De Camilli, P. (1996). A presynaptic inositol-5-phosphatase. Nature. https://doi.org/10.1038/379353a0

Merrifield, C. J., Perrais, D., \& Zenisek, D. (2005). Coupling between clathrin-coated-pit invagination, cortactin recruitment, and membrane scission observed in live cells. Cell. https://doi.org/10.1016/j.cell.2005.03.015

Mettlen, M., \& Danuser, G. (2014). Imaging and Modeling the Dynamics of Clathrin-Mediated Endocytosis. Cold Spring Harbor Perspectives in Biology, 6(12), a017038-a017038. https://doi.org/10.1101/cshperspect.a017038

Mettlen, Marcel, Chen, P.-H., Srinivasan, S., Danuser, G., \& Schmid, S. L. (2018). Regulation of Clathrin-Mediated Endocytosis. Annual Review of Biochemistry. https://doi.org/10.1146/annurevbiochem-062917-012644

Mettlen, Marcel, Loerke, D., Yarar, D., Danuser, G., \& Schmid, S. L. (2010a). Cargo- and adaptorspecific mechanisms regulate clathrin-mediated endocytosis. The Journal of Cell Biology, 188(6), 919-933. https://doi.org/10.1083/jcb.200908078

Mettlen, Marcel, Loerke, D., Yarar, D., Danuser, G., \& Schmid, S. L. (2010b). Cargo- and adaptorspecific mechanisms regulate clathrin-mediated endocytosis. The Journal of Cell Biology, 188(6), 919-933. https://doi.org/10.1083/jcb.200908078

Mettlen, Marcel, Stoeber, M., Loerke, D., Antonescu, C. N., Danuser, G., \& Schmid, S. L. (2009). Endocytic accessory proteins are functionally distinguished by their differential effects on the maturation of clathrin-coated pits. Molecular Biology of the Cell, 20(14), 3251-3260. https://doi.org/10.1091/mbc.E09-03-0256

Miettinen, P. J., Berger, J. E., Meneses, J., Phung, Y., Pedersen, R. A., Werb, Z., \& Derynck, R. (1995). Epithelial immaturity and multiorgan failure in mice lacking epidermal growth factor receptor. Nature. https://doi.org/10.1038/376337a0 
Mîinea, C. P., Sano, H., Kane, S., Sano, E., Fukuda, M., Peränen, J., .. Lienhard, G. E. (2005). AS160, the Akt substrate regulating GLUT4 translocation, has a functional Rab GTPase-activating protein domain. Biochemical Journal. https://doi.org/10.1042/bj20050887

Miled, N., Yan, Y., Hon, W. C., Perisic, O., Zvelebil, M., Inbar, Y., ... Williams, R. L. (2007). Mechanism of two classes of cancer mutations in the phosphoinositide 3-kinase catalytic subunit. Science. https://doi.org/10.1126/science.1135394

Mim, C., \& Unger, V. M. (2012). Membrane curvature and its generation by BAR proteins. Trends in Biochemical Sciences, 37(12), 526-533. https://doi.org/10.1016/J.TIBS.2012.09.001

Mineo, C., Gill, G. N., Richard, G., Anderson, W., \& Anderson, R. G. W. (1999). CELL BIOLOGY AND METABOLISM : Regulated Migration of Epidermal Growth Factor Receptor from Caveolae Regulated Migration of Epidermal Growth Factor Receptor from Caveolae *, 274(43), 3063630643. https://doi.org/10.1074/jbc.274.43.30636

Mishra, S. K., Agostinelli, N. R., Brett, T. J., Mizukami, I., Ross, T. S., \& Traub, L. M. (2001). Clathrinand AP-2-binding Sites in HIP1 Uncover a General Assembly Role for Endocytic Accessory Proteins. Journal of Biological Chemistry, 276(49), 46230-46236. https://doi.org/10.1074/jbc.M108177200

Modzelewska, K., Newman, L. P., Desai, R., \& Keely, P. J. (2006). Ack1 mediates Cdc42-dependent cell migration and signaling to p130 Cas. Journal of Biological Chemistry, 281(49), 37527-37535. https://doi.org/10.1074/jbc.M604342200

Molina, T. J., Kishihara, K., Siderovskid, D. P., Van Ewijk, W., Narendran, A., Timms, E., ... Mak, T. W. (1992). Profound block in thymocyte development in mice lacking p561ck. Nature. https://doi.org/10.1038/357161a0

Morgan, J. R., Prasad, K., Jin, S., Augustine, G. J., \& Lafer, E. M. (2001). Uncoating of clathrin-coated vesicles in presynaptic terminals: Roles for Hsc70 and auxilin. Neuron. https://doi.org/10.1016/S0896-6273(01)00467-6

Motley, A., Bright, N. A., Seaman, M. N. J., \& Robinson, M. S. (2003). Clathrin-mediated endocytosis in AP-2-depleted cells. Journal of Cell Biology, 162(5), 909-918. https://doi.org/10.1083/jcb.200305145

Mousavi, S. A., Malerød, L., Berg, T., \& Kjeken, R. (2004). Clathrin-dependent endocytosis. The Biochemical Journal, 377(Pt 1), 1-16. https://doi.org/10.1042/BJ20031000

Murphy, J. E., Padilla, B. E., Hasdemir, B., Cottrell, G. S., \& Bunnett, N. W. (2009). Endosomes: a legitimate platform for the signaling train. Proceedings of the National Academy of Sciences of the United States of America, 106(42), 17615-17622. https://doi.org/10.1073/pnas.0906541106

Nakajima, H., Ishikawa, Y., Furuya, M., Sano, T., Ohno, Y., Horiguchi, J., \& Oyama, T. (2014). Protein expression, gene amplification, and mutational analysis of EGFR in triple-negative breast cancer. Breast Cancer, 21(1), 66-74. https://doi.org/10.1007/s12282-012-0354-1

Nam, S., Kim, D., Cheng, J. Q., Zhang, S., Lee, J.-H., Buettner, R., ... Jove, R. (2005). Action of the Src family kinase inhibitor, dasatinib (BMS-354825), on human prostate cancer cells. Cancer Research, 65(20), 9185-9189. https://doi.org/10.1158/0008-5472.CAN-05-1731 
Nández, R., Balkin, D. M., Messa, M., Liang, L., Paradise, S., Czapla, H., ... De Camilli, P. (2014). A role of OCRL in clathrin-coated pit dynamics and uncoating revealed by studies of Lowe syndrome cells. ELife. https://doi.org/10.7554/eLife.02975

Näthke, I. S., Heuser, J., Lupas, A., Stock, J., Turck, C. W., \& Brodsky, F. M. (1992). Folding and trimerization of clathrin subunits at the triskelion hub. Cell. https://doi.org/10.1016/00928674(92)90033-9

Nautiyal, J., Majumder, P., Patel, B. B., Lee, F. Y., \& Majumdar, A. P. N. (2009). Src inhibitor dasatinib inhibits growth of breast cancer cells by modulating EGFR signaling. Cancer Letters, 283(2), 143 151. https://doi.org/10.1016/j.canlet.2009.03.035

Nygaard, H. B. (2018). Targeting Fyn Kinase in Alzheimer's Disease. Biological Psychiatry, 83(4), 369376. https://doi.org/10.1016/j.biopsych.2017.06.004

Offterdinger, M., \& Bastiaens, P. I. (2008). Prolonged EGFR signaling by ERBB2-mediated sequestration at the plasma membrane. Traffic. https://doi.org/10.1111/j.1600-0854.2007.00665.x

Ohno, H., Stewart, J., Fournier, M. C., Bosshart, H., Rhee, I., Miyatake, S., ... Bonifacino, J. S. (1995). Interaction of tyrosine-based sorting signals with clathrin-associated proteins. Science. https://doi.org/10.1126/science.7569928

Olusanya, O., Andrews, P. D., Swedlow, J. R., \& Smythe, E. (2001). Phosphorylation of threonine 156 of the $\mu 2$ subunit of the AP2 complex is essential for endocytosis in vitro and in vivo. Current Biology, 11(11), 896-900. https://doi.org/10.1016/S0960-9822(01)00240-8

Oneyama, C., Iino, T., Saito, K., Suzuki, K., Ogawa, A., \& Okada, M. (2009). Transforming Potential of Src Family Kinases Is Limited by the Cholesterol-Enriched Membrane Microdomain. Molecular and Cellular Biology. https://doi.org/10.1128/mcb.00941-09

Organ, S. L., \& Tsao, M. S. (2011). An overview of the c-MET signaling pathway. Therapeutic Advances in Medical Oncology. https://doi.org/10.1177/1758834011422556

Ouyang, M., Wan, R., Qin, Q., Peng, Q., Wang, P., Wu, J., ... Wang, Y. (2019). Sensitive FRET Biosensor Reveals Fyn Kinase Regulation by Submembrane Localization. ACS Sensors. https://doi.org/10.1021/acssensors.8b00896

Owen, D. J. (2002). A Structural Explanation for the Recognition of Tyrosine-Based Endocytotic Signals. Science, 282(5392), 1327-1332. https://doi.org/10.1126/science.282.5392.1327

Paige, L. A., Nadler, M. J. S., Harrison, M. L., Cassady, J. M., \& Geahlen, R. L. (1993). Reversible palmitoylation of the protein-tyrosine kinase p56(lck). Journal of Biological Chemistry.

Palacios, E. H., \& Weiss, A. (2004). Function of the Src-family kinases, Lck and Fyn, in T-cell development and activation. Oncogene, 23(48 REV. ISS. 7), 7990-8000. https://doi.org/10.1038/sj.onc.1208074

Pang, J. H., Kraemer, A., Stehbens, S. J., Frame, M. C., \& Yap, A. S. (2005). Recruitment of phosphoinositide 3-kinase defines a positive contribution of tyrosine kinase signaling to E-cadherin function. Journal of Biological Chemistry. https://doi.org/10.1074/jbc.M412148200 
Park, B.-C., Yim, Y.-I., Zhao, X., Olszewski, M. B., Eisenberg, E., \& Greene, L. E. (2015). The clathrinbinding and J-domains of GAK support the uncoating and chaperoning of clathrin by Hsc70 in the brain. Journal of Cell Science. https://doi.org/10.1242/jcs.171058

Parsons, S. J., \& Parsons, J. T. (2004). Src family kinases, key regulators of signal transduction. Oncogene, 23(48), 7906-7909. https://doi.org/10.1038/sj.onc.1208160

Pascolutti, R., Algisi, V., Conte, A., Raimondi, A., Pasham, M., Upadhyayula, S., ... Sigismund, S. (2019). Molecularly Distinct Clathrin-Coated Pits Differentially Impact EGFR Fate and Signaling. Cell Reports, 27(10), 3049-3061.e6. https://doi.org/10.1016/j.celrep.2019.05.017

Paz-Ares, L., Soulières, D., Melezínek, I., Moecks, J., Keil, L., Mok, T., ... Klughammer, B. (2010). Clinical outcomes in non-small-cell lung cancer patients with EGFR mutations: Pooled analysis. Journal of Cellular and Molecular Medicine. https://doi.org/10.1111/j.1582-4934.2009.00991.x

Peng, X. ding, Xu, P. Z., Chen, M. L., Hahn-Windgassen, A., Skeen, J., Jacobs, J., ... Hay, N. (2003). Dwarfism, impaired skin development, skeletal muscle atrophy, delayed bone development, and impeded adipogenesis in mice lacking Akt1 and Akt2. Genes and Development.

Pennock, S., \& Wang, Z. (2003). Stimulation of Cell Proliferation by Endosomal Epidermal Growth Factor Receptor As Revealed through Two Distinct Phases of Signaling. Molecular and Cellular Biology. https://doi.org/10.1128/mcb.23.16.5803-5815.2003

Petrelli, A., Gilestro, G. F., Lanzardo, S., Comoglio, P. M., Migone, N., \& Giordano, S. (2002). The endophilin-CIN85-Cbl complex mediates ligand-dependent downregulation of c-Met. Nature, 416(6877), 187-190. https://doi.org/10.1038/416187a

Platta, H. W., \& Stenmark, H. (2011). Endocytosis and signaling. Current Opinion in Cell Biology. https://doi.org/10.1016/j.ceb.2011.03.008

Pohlmann, P. R., Mayer, I. A., \& Mernaugh, R. (2009). Resistance to trastuzumab in breast cancer. Clinical Cancer Research. https://doi.org/10.1158/1078-0432.CCR-09-0636

Ponzo, M. G., \& Park, M. (2010). The met receptor tyrosine kinase and basal breast cancer. Cell Cycle. https://doi.org/10.4161/cc.9.6.11033

Prashar, A., Bhatia, S., Gigliozzi, D., Martin, T., Duncan, C., Guyard, C., \& Terebiznik, M. R. (2013). Filamentous morphology of bacteria delays the timing of phagosome morphogenesis in macrophages. The Journal of Cell Biology, 203(6), 1081-1097. https://doi.org/10.1083/jcb.201304095

Prashar, A., Ortiz, M. E., Lucarelli, S., Barker, E., Tabatabeiyazdi, Z., Shamoun, F., Raju, D., Antonescu, C. N., Guyard, C. \& Terebiznik, M. R. (2018). Small Rho GTPases and the Effector VipA Mediate the Invasion of Epithelial Cells by Filamentous Legionella pneumophila. Frontiers in Cellular and Molecular Biology, 8 (133).

Prieto-Echagüe, V., Gucwa, A., Craddock, B. P., Brown, D. a., \& Miller, W. T. (2010). Cancer-associated mutations activate the nonreceptor tyrosine kinase Ack1. Journal of Biological Chemistry, 285(14), 10605-10615. https://doi.org/10.1074/jbc.M109.060459

Prieto-Echagüe, V., \& Miller, W. T. (2011). Regulation of ack-family nonreceptor tyrosine kinases. 
Journal of Signal Transduction, 2011, 742372. https://doi.org/10.1155/2011/742372

Puthenveedu, M. A., \& von Zastrow, M. (2006). Cargo regulates clathrin-coated pit dynamics. Cell, 127(1), 113-124. https://doi.org/10.1016/j.cell.2006.08.035

Qu, Y., Han, B., Yu, Y., Yao, W., Bose, S., Karlan, B. Y., ... Cui, X. (2015). Evaluation of MCF10A as a reliable model for normal human mammary epithelial cells. PLOS ONE.

https://doi.org/10.1371/journal.pone.0131285

Rajadurai, C. V., Havrylov, S., Zaoui, K., Vaillancourt, R., Stuible, M., Naujokas, M., ... Park, M. (2012). Met receptor tyrosine kinase signals through a cortactin-Gab1 scaffold complex, to mediate invadopodia. Journal of Cell Science. https://doi.org/10.1242/jcs.100834

Rajagopalan, V., Zucker, I. H., Jones, J. A., Carlson, M., \& Ma, Y. J. (2008). Cardiac ErbB-1/ErbB-2 mutant expression in young adult mice leads to cardiac dysfunction. American Journal of Physiology-Heart and Circulatory Physiology. https://doi.org/10.1152/ajpheart.91436.2007

Rappoport, J. Z., Benmerah, A., \& Simon, S. M. (2005). Analysis of the AP-2 adaptor complex and cargo during clathrin-mediated endocytosis. Traffic. https://doi.org/10.1111/j.1600-0854.2005.00280.x

Ravid, T., Sweeney, C., Gee, P., Carraway, K. L., \& Goldkorn, T. (2002). Epidermal growth factor receptor activation under oxidative stress fails to promote c-Cbl mediated down-regulation. Journal of Biological Chemistry. https://doi.org/10.1074/jbc.M204677200

Red Brewer, M., Yun, C.-H., Lai, D., Lemmon, M. A., Eck, M. J., \& Pao, W. (2013). Mechanism for activation of mutated epidermal growth factor receptors in lung cancer. Proceedings of the National Academy of Sciences. https://doi.org/10.1073/pnas.1220050110

Res, M. P. B. B., Prasad, K., \& Lippoldt, R. E. (1988). Molecular Characterization of the AP180 Coated Vesicle Assembly Protein? Assembly, (1987), 6098-6104.

Resh, M. D. (1994). Myristylation and palmitylation of Src family members: The fats of the matter. Cell, 76(3), 411-413. https://doi.org/10.1016/0092-8674(94)90104-X

Rimawi, M. F., Schiff, R., \& Osborne, C. K. (2015). Targeting HER2 for the Treatment of Breast Cancer. Annual Review of Medicine. https://doi.org/10.1146/annurev-med-042513-015127

Ringerike, T., Blystad, F. D., Levy, F. O., Madshus, I. H., \& Stang, E. (2002). Cholesterol is important in control of EGF receptor kinase activity but EGF receptors are not concentrated in caveolae. Journal of Cell Science, 115, 1331-1340.

Rodgers, J. T., Vogel, R. O., \& Puigserver, P. (2011). Clk2 and B56 $\beta$ Mediate Insulin-Regulated Assembly of the PP2A Phosphatase Holoenzyme Complex on Akt. Molecular Cell. https://doi.org/10.1016/j.molcel.2011.02.007

Rodrigues, G. A., Falasca, M., Zhang, Z., Ong, S. H., \& Schlessinger, J. (2000). A novel positive feedback loop mediated by the docking protein Gab1 and phosphatidylinositol 3-kinase in epidermal growth factor receptor signaling. Molecular and Cellular Biology, 20(4), 1448-1459.

Roepstorff, K., Thomsen, P., Sandvig, K., \& Van Deurs, B. (2002). Sequestration of epidermal growth factor receptors in non-caveolar lipid rafts inhibits ligand binding. Journal of Biological Chemistry, 
277(21), 18954-18960. https://doi.org/10.1074/jbc.M201422200

Roskoski, R. (2004). Src protein-tyrosine kinase structure and regulation. Biochemical and Biophysical Research Communications, 324(4), 1155-1164. https://doi.org/10.1016/j.bbrc.2004.09.171

Roskoski, R. (2014). The ErbB/HER family of protein-tyrosine kinases and cancer. Pharmacological Research, 79, 34-74. Retrieved from http://www.sciencedirect.com/science/article/pii/S1043661813001771

Rosselli-Murai, L. K., Yates, J. A., Yoshida, S., Bourg, J., Ho, K. K. Y., White, M., ... Liu, A. P. (2018). Loss of PTEN promotes formation of signaling-capable clathrin-coated pits. Journal of Cell Science, 131(8), jcs208926. https://doi.org/10.1242/jcs.208926

Royle, S. J. (2006). The cellular functions of clathrin. Cellular and Molecular Life Sciences, 63(16), 1823-1832. https://doi.org/10.1007/s00018-005-5587-0

Rush, J. S., Quinalty, L. M., Engelman, L., Sherry, D. M., \& Ceresa, B. P. (2012). Endosomal accumulation of the activated epidermal growth factor receptor (EGFR) induces apoptosis. Journal of Biological Chemistry. https://doi.org/10.1074/jbc.M111.294470

Saito, Y. D., Jensen, A. R., Salgia, R., \& Posadas, E. M. (2010). Fyn: A novel molecular target in cancer. Cancer, 116(7), 1629-1637. https://doi.org/10.1002/cncr.24879

Sandilands, E., Brunton, V. G., \& Frame, M. C. (2007). The membrane targeting and spatial activation of Src, Yes and Fyn is influenced by palmitoylation and distinct RhoB/RhoD endosome requirements. Journal of Cell Science. https://doi.org/10.1242/jcs.003657

Sano, H., Kane, S., Sano, E., Mîinea, C. P., Asara, J. M., Lane, W. S., ... Lienhard, G. E. (2003). Insulinstimulated Phosphorylation of a Rab GTPase-activating Protein Regulates GLUT4 Translocation. Journal of Biological Chemistry, 278(17), 14599-14602. https://doi.org/10.1074/jbc.C300063200

Sarbassov, D. D., Guertin, D. A., Ali, S. M., \& Sabatini, D. M. (2005). Phosphorylation and regulation of Akt/PKB by the rictor-mTOR complex. Science. https://doi.org/10.1126/science.1106148

Sato, I., Obata, Y., Kasahara, K., Nakayama, Y., Fukumoto, Y., Yamasaki, T., ... Yamaguchi, N. (2009). Differential trafficking of Src, Lyn, Yes and Fyn is specified by the state of palmitoylation in the SH4 domain. Journal of Cell Science. https://doi.org/10.1242/jcs.034843

Sato, K.-I. (2013). Cellular functions regulated by phosphorylation of EGFR on Tyr845. International Journal of Molecular Sciences, 14(6), 10761-10790. https://doi.org/10.3390/ijms140610761

Sawada, K., Radjabi, A. R., Shinomiya, N., Kistner, E., Kenny, H., Becker, A. R., ... Lengyel, E. (2007). c-Met overexpression is a prognostic factor in ovarian cancer and an effective target for inhibition of peritoneal dissemination and invasion. Cancer Research. https://doi.org/10.1158/0008-5472.CAN06-1147

Saxton, R. A., \& Sabatini, D. M. (2017). mTOR Signaling in Growth, Metabolism, and Disease. Cell. https://doi.org/10.1016/j.cell.2017.02.004

Schmid, S. L. (2017). Reciprocal regulation of signaling and endocytosis: Implications for the evolving cancer cell. Journal of Cell Biology. https://doi.org/10.1083/jcb.201705017 
Schmidt, C., Bladt, F., Goedecke, S., Brinkmann, V., Zschiesche, W., Sharpe, M., ... Goedecke, S. (1995). Scatter factor/hepatocyte growth factor is essential for liver development. Nature. https://doi.org/10.1038/373699a0

Scott, B. L., Sochacki, K. A., Low-Nam, S. T., Bailey, E. M., Luu, Q. A., Hor, A., ... Hoppe, A. D. (2018). Membrane bending occurs at all stages of clathrincoat assembly and defines endocytic dynamics. Nature Communications. https://doi.org/10.1038/s41467-018-02818-8

Seykora, J. T., Mei, L., Dotto, G. P., \& Stein, P. L. (2002). 'Srcasm: a novel Src activating and signaling molecule. The Journal of Biological Chemistry, 277(4), 2812-2822.

https://doi.org/10.1074/jbc.M106813200

Shen, F., Lin, Q., Gu, Y., Childress, C., \& Yang, W. (2007). Activated Cdc42-associated Kinase 1 Is a Component of EGF Receptor Signaling Complex and Regulates EGF Receptor Degradation, 18(March), 732-742. https://doi.org/10.1091/mbc.E06

Shen, H., Ferguson, S. M., Dephoure, N., Park, R., Yang, Y., Volpicelli-Daley, L., ... De Camilli, P. (2011). Constitutive activated Cdc42-associated kinase (Ack) phosphorylation at arrested endocytic clathrin-coated pits of cells that lack dynamin. Molecular Biology of the Cell, 22(4), 493-502. https://doi.org/10.1091/mbc.E10-07-0637

Sigismund, S., Avanzato, D., \& Lanzetti, L. (2018). Emerging functions of the EGFR in cancer. Molecular Oncology, 12(1), 3-20. https://doi.org/10.1002/1878-0261.12155

Slepnev, V. I., \& De Camilli, P. (2000). Accessory factors in clathrin-dependent synaptic vesicle endocytosis. Nature Reviews Neuroscience. https://doi.org/10.1038/35044540

Sochacki, K. A., Dickey, A. M., Strub, M. P., \& Taraska, J. W. (2017). Endocytic proteins are partitioned at the edge of the clathrin lattice in mammalian cells. Nature Cell Biology.

https://doi.org/10.1038/ncb3498

Soriano, P., Montgomery, C., Geske, R., \& Bradley, A. (1991). Targeted disruption of the c-src protooncogene leads to osteopetrosis in mice. Cell. https://doi.org/10.1016/0092-8674(91)90499-O

Sorkin, A., \& Goh, L. K. (2009). Endocytosis and intracellular trafficking of ErbBs. Experimental Cell Research. https://doi.org/10.1016/j.yexcr.2008.07.029

Sorkin, A., \& Puthenveedu, M. A. (2013). Clathrin-Mediated Endocytosis. In Vesicle Trafficking in Cancer (pp. 1-31). New York, NY: Springer New York. https://doi.org/10.1007/978-1-4614-65287 1

Sorkina, T., Huang, F., Beguinot, L., \& Sorkin, A. (2002). Effect of tyrosine kinase inhibitors on clathrincoated pit recruitment and internalization of epidermal growth factor receptor. The Journal of Biological Chemistry, 277(30), 27433-27441. https://doi.org/10.1074/jbc.M201595200

Soubeyran, P., Kowanetz, K., Szymkiewicz, I., Langdon, W. Y., \& Dikic, I. (2002). Cbl-CIN85endophilin complex mediates ligand-induced downregulation of EGF receptors. Nature, 416(6877), 183-187. https://doi.org/10.1038/416183a

Sousa, L. P., Lax, I., Shen, H., Ferguson, S. M., Camilli, P. D., \& Schlessinger, J. (2012). Suppression of 
EGFR endocytosis by dynamin depletion reveals that EGFR signaling occurs primarily at the plasma membrane. Proceedings of the National Academy of Sciences.

https://doi.org/10.1073/pnas.1200164109

Stahl, J. M., Sharma, A., Cheung, M., Zimmerman, M., Cheng, J. Q., Bosenberg, M. W., ... Robertson, G. P. (2004). Deregulated Akt3 activity promotes development of malignant melanoma. Cancer Research. https://doi.org/10.1158/0008-5472.CAN-04-1399

Stål, O., Pérez-Tenorio, G., Åkerberg, L., Olsson, B., Nordenskjöld, B., Skoog, L., \& Rutqvist, L. E. (2003). Akt kinases in breast cancer and the results of adjuvant therapy. Breast Cancer Research. https://doi.org/10.1186/bcr569

Stambolic, V., Suzuki, A., De la Pompa, J. L., Brothers, G. M., Mirtsos, C., Sasaki, T., ... Mak, T. W. (1998). Negative regulation of PKB/Akt-dependent cell survival by the tumor suppressor PTEN. Cell. https://doi.org/10.1016/S0092-8674(00)81780-8

Stanishneva-Konovalova, T. B., Derkacheva, N. I., Polevova, S. V., \& Sokolova, O. S. (2016). The Role of BAR Domain Proteins in the Regulation of Membrane Dynamics. Acta Naturae.

Stasyk, T., Schiefermeier, N., Skvortsov, S., Zwierzina, H., Peränen, J., Bonn, G. K., \& Huber, L. A. (2007). Identification of Endosomal Epidermal Growth Factor Receptor Signaling Targets by Functional Organelle Proteomics. Molecular \& Cellular Proteomics. https://doi.org/10.1074/mcp.m600463-mcp200

Stein, P. L., Vogel, H., \& Soriano, P. (1994). Combined deficiencies of src, fyn, and yes tyrosine kinases in mutant mice. Genes and Development. https://doi.org/10.1101/gad.8.17.1999

Stephens, L., Anderson, K., Stokoe, D., Erdjument-Bromage, H., Painter, G. F., Holmes, A. B., ... Hawkins, P. T. (1998). Prohtein kinase B kinases that mediate phosphatidylinositol 3,4,5trisphosphate-dependent activation of protein kinase B. Science. https://doi.org/10.1126/science.279.5351.710

Stoddart, A., Dykstra, M. L., Brown, B. K., Song, W., Pierce, S. K., \& Brodsky, F. M. (2002). Lipid rafts unite signaling cascades with clathrin to regulate BCR internalization. Immunity. https://doi.org/10.1016/S1074-7613(02)00416-8

Sugiyama, M. G., Fairn, G. D., \& Antonescu, C. N. (2019). Akt-ing up just about everywhere: Compartment-specific Akt activation and function in receptor tyrosine kinase signaling. Frontiers in Cell and Developmental Biology, 7(May), 70. https://doi.org/10.3389/FCELL.2019.00070

Sundborger, A. C., \& Hinshaw, J. E. (2014). Regulating dynamin dynamics during endocytosis. F1000Prime Reports. https://doi.org/10.12703/p6-85

Sweeney, C., \& Carraway, K. L. (2000). Ligand discrimination by ErbB receptors: differential signaling through differential phosphorylation site usage. Oncogene, 19(49), 5568-5573. https://doi.org/10.1038/sj.onc.1203913

Take, H., Watanabe, S., Takeda, K., Yu, Z. X., Iwata, N., \& Kajigaya, S. (2000). Cloning and characterization of a novel adaptor protein, CIN85, that interacts with c-Cbl. Biochemical and Biophysical Research Communications, 268(2), 321-328. https://doi.org/10.1006/bbrc.2000.2147 
Tarcic, G., Boguslavsky, S. K., Wakim, J., Kiuchi, T., Liu, A., Reinitz, F., .. Yarden, Y. (2009). An Unbiased Screen Identifies DEP-1 Tumor Suppressor as a Phosphatase Controlling EGFR Endocytosis. Current Biology. https://doi.org/10.1016/j.cub.2009.09.048

Taub, N., Teis, D., Ebner, H. L., Hess, M. W., \& Huber, L. A. (2007). Late Endosomal Traffic of the Epidermal Growth Factor Receptor Ensures Spatial and Temporal Fidelity of Mitogen-activated Protein Kinase Signaling. Molecular Biology of the Cell. https://doi.org/10.1091/mbc.e07-02-0098

Taylor, M. J., Perrais, D., \& Merrifield, C. J. (2011). A high precision survey of the molecular dynamics of Mammalian clathrin-mediated endocytosis. PLoS Biology, 9(3), e1000604. https://doi.org/10.1371/journal.pbio.1000604

Tebar, F., Bohlander, S. K., \& Sorkin, a. (1999). Clathrin assembly lymphoid myeloid leukemia (CALM) protein: localization in endocytic-coated pits, interactions with clathrin, and the impact of overexpression on clathrin-mediated traffic. Molecular Biology of the Cell, 10(8), 2687-2702. https://doi.org/10.1091/mbc.10.8.2687

Teo, M., Tan, L., Lim, L., \& Manser, E. (2001). The tyrosine kinase ACK1 associates with clathrincoated vesicles through a binding motif shared by arrestin and other adaptors. The Journal of Biological Chemistry, 276(21), 18392-18398. https://doi.org/10.1074/jbc.M008795200

The Neuregulin-I/ErbB Signaling System in Development and Disease. (2007). The Neuregulin-I/ErbB Signaling System in Development and Disease. https://doi.org/10.1007/978-3-540-37107-6

Thomas, A. J. E., Soriano, P., \& Brugge, J. S. (1991). Phosphorylation of c-Src on Tyrosine 527 by Another Protein Tyrosine Kinase. Advancement Of Science, 254(5031), 568-571.

Thompson, D. M., \& Gill, G. N. (1985). The EGF receptor: structure, regulation and potential role in malignancy. Cancer Surveys.

Tice, D. A., Biscardi, J. S., Nickles, A. L., \& Parsons, S. J. (2002). Mechanism of biological synergy between cellular Src and epidermal growth factor receptor. Proceedings of the National Academy of Sciences. https://doi.org/10.1073/pnas.96.4.1415

Tomas, A., Futter, C. E., \& Eden, E. R. (2014). EGF receptor trafficking: Consequences for signaling and cancer. Trends in Cell Biology, 24(1), 26-34. https://doi.org/10.1016/j.tcb.2013.11.002

Tong, J., Taylor, P., \& Moran, M. F. (2014). Proteomic Analysis of the Epidermal Growth Factor Receptor (EGFR) Interactome and Post-translational Modifications Associated with Receptor Endocytosis in Response to EGF and Stress. Molecular \& Cellular Proteomics. https://doi.org/10.1074/mcp.m114.038596

Tosoni, D., Puri, C., Confalonieri, S., Salcini, A. E., De Camilli, P., Tacchetti, C., \& Di Fiore, P. P. (2005). TTP specifically regulates the internalization of the transferrin receptor. Cell, 123(5), 875888. https://doi.org/10.1016/j.cell.2005.10.021

Traub, L. M. (2009). Tickets to ride: Selecting cargo for clathrin-regulated internalization. Nature Reviews Molecular Cell Biology, 10(9), 583-596. https://doi.org/10.1038/nrm2751

Traub, L. M., \& Bonifacino, J. S. (2013). Cargo recognition in clathrin-mediated endocytosis. Cold 
Spring Harbor Perspectives in Biology. https://doi.org/10.1101/cshperspect.a016790

Trotman, L. C., Wang, X., Alimonti, A., Chen, Z., Teruya-Feldstein, J., Yang, H., .. Pandolfi, P. P. (2007). Ubiquitination Regulates PTEN Nuclear Import and Tumor Suppression. Cell. https://doi.org/10.1016/j.cell.2006.11.040

Tschopp, O. (2005). Essential role of protein kinase B (PKB /Akt3) in postnatal brain development but not in glucose homeostasis. Development. https://doi.org/10.1242/dev.01864

Ueda, Y., Igishi, T., Hashimoto, K., Suyama, H., Araki, K., Sumikawa, T., ... Shimizu, E. (2009). Synergistic cell growth inhibition by the combination of amrubicin and Akt-suppressing tyrosine kinase inhibitors in small cell lung cancer cells: Implication of c-Src and its inhibitor. International Journal of Oncology, 34(3), 941-953. https://doi.org/10.3892/ijo_00000195

Ungewickell, A., Ward, M. E., Ungewickell, E., \& Majerus, P. W. (2004). The inositol polyphosphate 5phosphatase Ocrl associates with endosomes that are partially coated with clathrin. Proceedings of the National Academy of Sciences. https://doi.org/10.1073/pnas.0405664101

Ungewickell, E., Ungewickell, H., Holstein, S., Lindner, R., Prasad, K., Barouch, W., ... Eisenberg, E. (1995). Role of auxilin in uncoating clathrin-coated vesicles. Nature, 378, 632-635.

Van't Hof, W., \& Resh, M. D. (2000). Dual fatty acylation of p59(Fyn) is required for association with the T cell receptor $\zeta$ chain through phosphotyrosine-Src homology domain-2 interactions. Journal of Cell Biology. https://doi.org/10.1083/jcb.145.2.377

van der Vos, K. E., \& Coffer, P. J. (2010). The Extending Network of FOXO Transcriptional Target Genes. Antioxidants \& Redox Signaling. https://doi.org/10.1089/ars.2010.3419

VANHAESEBROECK, B., \& ALESSI, D. R. (2000). The PI3K-PDK1 connection: more than just a road to PKB. Biochemical Journal. https://doi.org/10.1042/bj3460561

Vansteenkiste, J., \& Wauters, E. (2018). Tyrosine kinase inhibition of EGFR: A successful history of targeted therapy for NSCLC since 20 years. Annals of Oncology.

https://doi.org/10.1093/annonc/mdx724

Varshavsky, A. (1997). The ubiquitin system. Trends in Biochemical Sciences. https://doi.org/10.1016/S0968-0004(97)01122-5

Vassilopoulos, S., Esk, C., Hoshino, S., Funke, B. H., Chen, C. Y., Plocik, A. M., ... Brodsky, F. M. (2009). A Role for the CHC22 clathrin heavy-chain isoform in human glucose metabolism. Science. https://doi.org/10.1126/science.1171529

Verstreken, P., Koh, T. W., Schulze, K. L., Zhai, R. G., Hiesinger, P. R., Zhou, Y., ... Bellen, H. J. (2003). Synaptojanin is recruited by endophilin to promote synaptic vesicle uncoating. Neuron. https://doi.org/10.1016/S0896-6273(03)00644-5

Vieira, A. V., Lamaze, C., \& Schmid, S. L. (1996). Control of EGF receptor signaling by clathrinmediated endocytosis. Science. https://doi.org/10.1126/science.274.5295.2086

VIEIRA, O. V., BOTELHO, R. J., \& GRINSTEIN, S. (2002). Phagosome maturation: aging gracefully. Biochemical Journal. https://doi.org/10.1042/bj20020691 
Von Kleist, L., Stahlschmidt, W., Bulut, H., Gromova, K., Puchkov, D., Robertson, M. J., ... Haucke, V. (2011). Role of the clathrin terminal domain in regulating coated pit dynamics revealed by small molecule inhibition. Cell, 146(3), 471-484. https://doi.org/10.1016/j.cell.2011.06.025

Wada, I., Lai, W. H., Posner, B. I., \& Bergeron, J. J. M. (1992). Association of the tyrosine phosphorylated epidermal growth factor receptor with a 55-kD tyrosine phosphorylated protein at the cell surface and in endosomes. Journal of Cell Biology. https://doi.org/10.1083/jcb.116.2.321

Wallroth, A., \& Haucke, V. (2018). Phosphoinositide conversion in endocytosis and the endolysosomal system. Journal of Biological Chemistry. https://doi.org/10.1074/jbc.R117.000629

Wang, Y., Pennock, S., Chen, X., \& Wang, Z. (2002a). Endosomal signaling of epidermal growth factor receptor stimulates signal transduction pathways leading to cell survival. Mol Cell Biol, 22(20), 7279-7290. https://doi.org/10.1128/MCB.22.20.7279

Wang, Y., Pennock, S., Chen, X., \& Wang, Z. (2002b). Endosomal Signaling of Epidermal Growth Factor Receptor Stimulates Signal Transduction Pathways Leading to Cell Survival. Molecular and Cellular Biology. https://doi.org/10.1128/mcb.22.20.7279-7290.2002

Wee, P., \& Wang, Z. (2017). Epidermal Growth Factor Receptor Cell Proliferation Signaling Pathways. Cancers, 9(5), 52. https://doi.org/10.3390/cancers9050052

Weidner, K. M., Di Cesare, S., Sachs, M., Brinkmann, V., Behrens, J., \& Birchmeier, W. (1996). Interaction between Gab1 and the c-Met receptor tyrosine kinase is responsible for epithelial morphogenesis. Nature. https://doi.org/10.1038/384173a0

Wendland, B. (2002). Epsins: Adaptors in endocytosis? Nature Reviews Molecular Cell Biology. https://doi.org/10.1038/nrm970

Wenk, M. R., Pellegrini, L., Klenchin, V. A., Di Paolo, G., Chang, S., Daniell, L., ... De Camilli, P. (2001). PIP kinase I $\gamma$ is the major PI(4,5)P2 synthesizing enzyme at the synapse. Neuron. https://doi.org/10.1016/S0896-6273(01)00456-1

Whiteman, E. L., Cho, H., \& Birnbaum, M. J. (2002). Role of Akt/protein kinase B in metabolism. Trends in Endocrinology and Metabolism, 13(10), 444-451. https://doi.org/10.1016/S1043-2760(02)006628

Wilde, A., Beattie, E. C., Lem, L., Riethof, D. A., Liu, S.-H., Mobley, W. C., ... Brodsky, F. M. (1999). EGF Receptor Signaling Stimulates SRC Kinase Phosphorylation of Clathrin, Influencing Clathrin Redistribution and EGF Uptake. Cell, 96(5), 677-687. https://doi.org/10.1016/S00928674(00)80578-4

Wolven, A., Okamura, H., Rosenblatt, Y., \& Resh, M. D. (1997). Palmitoylation of p59fyn is reversible and sufficient for plasma membrane association. Molecular Biology of the Cell. https://doi.org/10.1091/mbc.8.6.1159

Wong, K. A., Wilson, J., Russo, A., Wang, L., Okur, M. N., Wang, X., ... O’Bryan, J. P. (2012). Intersectin (ITSN) family of scaffolds function as molecular hubs in protein interaction networks. PLoS ONE. https://doi.org/10.1371/journal.pone.0036023 
Wu, C. J., O’Rourke, D. M., Feng, G. S., Johnson, G. R., Wang, Q., \& Greene, M. I. (2001). The tyrosine phosphatase SHP-2 is required for mediating phosphatidylinositol 3-kinase/Akt activation by growth factors. Oncogene, 20(42), 6018-6025. https://doi.org/10.1038/sj.onc.1204699

Wu, S., Majeed, S. R., Evans, T. M., Camus, M. D., Wong, N. M. L., Schollmeier, Y., ... Brodsky, F. M. (2016). Clathrin light chains' role in selective endocytosis influences antibody isotype switching. Proceedings of the National Academy of Sciences. https://doi.org/10.1073/pnas.1611189113

Yang, W, Lo, C. G., Dispenza, T., \& Cerione, R. a. (2001). The Cdc42 target ACK2 directly interacts with clathrin and influences clathrin assembly. The Journal of Biological Chemistry, 276(20), 17468-17473. https://doi.org/10.1074/jbc.M010893200

Yang, Wannian, \& Cerione, R. a. (1997). Cloning and characterization of a novel Cdc42-associated tyrosine kinase, ACK-2, from bovine brain. Journal of Biological Chemistry, 272(40), 2481924824. https://doi.org/10.1074/jbc.272.40.24819

Yang, X. D., Jia, X. C., Corvalan, J. R. F., Wang, P., \& Davis, C. G. (2001). Development of ABX-EGF, a fully human anti-EGF receptor monoclonal antibody, for cancer therapy. Critical Reviews in Oncology/Hematology. https://doi.org/10.1016/S1040-8428(00)00134-7

Yang, Z.-Z., Tschopp, O., Di-Poi, N., Bruder, E., Baudry, A., Dummler, B., ... Hemmings, B. A. (2005). Dosage-Dependent Effects of Akt1/Protein Kinase B (PKB ) and Akt3/PKB on Thymus, Skin, and Cardiovascular and Nervous System Development in Mice. Molecular and Cellular Biology. https://doi.org/10.1128/mcb.25.23.10407-10418.2005

Yao, Z., Darowski, K., St-Denis, N., Wong, V., Offensperger, F., Villedieu, A., ... Stagljar, I. (2017). A Global Analysis of the Receptor Tyrosine Kinase-Protein Phosphatase Interactome. Molecular Cell, 65(2), 347-360. https://doi.org/10.1016/j.molcel.2016.12.004

Yarden, Y., \& Pines, G. (2012). The ERBB network: At last, cancer therapy meets systems biology. Nature Reviews Cancer. https://doi.org/10.1038/nrc3309

Yatabe, Y., \& Mitsudomi, T. (2007). Epidermal growth factor receptor mutations in lung cancers. Pathology International. https://doi.org/10.1111/j.1440-1827.2007.02098.x

Zhang, B., Zerubia, J., \& Olivo-Marin, J.-C. (2007). Gaussian approximations of fluorescence microscope point-spread function models. Applied Optics, 46(10), 1819. https://doi.org/10.1364/ao.46.001819

Zhang, J., Ferguson, S. S., Barak, L. S., Ménard, L., \& Caron, M. G. (1996). Dynamin and beta-arrestin reveal distinct mechanisms for $\mathrm{G}$ protein-coupled receptor internalization. The Journal of Biological Chemistry, 271(31), 18302-18305. https://doi.org/10.1074/jbc.271.31.18302

Zhang, S. Q., Tsiaras, W. G., Araki, T., Wen, G., Minichiello, L., Klein, R., \& Neel, B. G. (2002). Receptor-specific regulation of phosphatidylinositol 3'-kinase activation by the protein tyrosine phosphatase Shp2. Molecular and Cellular Biology.

Zhang, X., Gureasko, J., Shen, K., Cole, P. A., \& Kuriyan, J. (2006). An Allosteric Mechanism for Activation of the Kinase Domain of Epidermal Growth Factor Receptor. Cell. https://doi.org/10.1016/j.cell.2006.05.013 
Zimmerman, B., Simaan, M., Lee, M. H., Luttrell, L. M., \& Laporte, S. A. (2009). c-Src-mediated phosphorylation of AP-2 reveals a general mechanism for receptors internalizing through the clathrin pathway. Cellular Signalling, 21(1), 103-110. https://doi.org/10.1016/j.cellsig.2008.09.013 